Supporting Information for

\title{
E-Selective Synthesis and Coordination Chemistry of Pyridine- Phosphaalkenes: Five Ligands Produce Four Distinct Types of Ru(II) Complexes
}

Mika L. Nakashige, ${ }^{\dagger}$ Jarin I.P. Loristo, ${ }^{\dagger, \#}$ Lesley S. Wong, ${ }^{\dagger, \#}$ Joshua R. Gurr, ${ }^{\dagger}$ Timothy J. O’Donnell, ${ }^{\dagger}$ Wesley Y. Yoshida, ${ }^{\dagger}$ Arnold L. Rheingold, ${ }^{£}$ Russell P. Hughes, ${ }^{\ddagger}$ and Matthew F. Cain ${ }^{\dagger, *}$

†Department of Chemistry, University of Hawai'i at Mānoa, 2545 McCarthy Mall, Honolulu, Hawaii 96822, United States

$\$ 6128$ Burke Laboratory, Department of Chemistry, Dartmouth College, Hanover, New Hampshire 03755, United States

${ }^{£}$ Department of Chemistry, University of California, San Diego, 9500 Gilman Drive, La Jolla, California 92093, United States

1. Complete Experimental Details for $\mathbf{2 b - 2 e , 3 b / d , ~ 4 b / d / e , ~ 5 a , ~ a n d ~} \mathbf{6 c}$ including their NMR spectra (page S2-S55)

2. DFT Computation Details (S56 - S57)

3. Experimental Details for Crystal Structure Refinement and Acquisition (S58 - S174) 
1. Complete Experimental Details for $\mathbf{2 b}-\mathbf{2 e}, 3 \mathbf{b} / \mathbf{d}, \mathbf{4 b} / \mathbf{d} / \mathbf{e}, 5 \mathbf{a}$, and $\mathbf{6 c}$ including their NMR spectra

General Experimental Details. Unless otherwise specified, all reactions and manipulations were performed under a nitrogen atmosphere in a MBraun glovebox or using standard Schlenk techniques. All glassware was oven-dried overnight (at minimum) at $140{ }^{\circ} \mathrm{C}$ prior to use. Anhydrous solvents were purchased directly from chemical suppliers (Aldrich or Acros), pumped directly into the glove box, and stored over oven-activated 4 or $5 \AA$ molecular sieves (Aldrich). $\mathrm{RuCl}_{2}\left(\mathrm{PPh}_{3}\right)_{3}$ and $\mathrm{Zn}$ dust were purchased from Strem and used as is; $\mathrm{PMe}_{3}$ was also bought from Strem and stored over molecular sieves prior to use. The 6-substituted pyridine-2carboxyaldehyde derivatives were obtained from Sigma Aldrich, and $\mathrm{Mes}^{*} \mathrm{PCl}_{2}$ was prepared via literature protocol. ${ }^{56} \mathrm{NMR}$ spectra were obtained on either Varian spectrometers operating at 300, 400, or $500 \mathrm{MHz}$; all spectra are displayed in the Supporting Information. NMR chemical shifts are reported as ppm relative to tetramethylsilane and are referenced to the residual proton or ${ }^{13} \mathrm{C}$ signal of the solvent $\left({ }^{1} \mathrm{H} \mathrm{CDCl}_{3}, 7.27 \mathrm{ppm} ;{ }^{1} \mathrm{H} \mathrm{C}_{6} \mathrm{D}_{6}, 7.16 \mathrm{ppm} ;{ }^{13} \mathrm{C} \mathrm{CDCl}_{3}, 77.16 \mathrm{ppm}\right.$; $\left.{ }^{13} \mathrm{C} \mathrm{C}_{6} \mathrm{D}_{6}, 128.06 \mathrm{ppm}\right)$. Mass spectroscopic data was collected on an Agilent 6545 AccurateMass Q-TOF LC/MS (NSF CHE-1532310). Analytical data were obtained from CENTC Elemental Analysis Facility at the University of Rochester, funded by NSF CHE-065-456. All Xray quality crystals were analyzed at the Small Molecular X-ray Crystallography Facility located at the University of California, San Diego.

Synthesis of Pyridine-Phosphaalkenes of Type 2. Derivative 2a was previously prepared as an $E / Z$ mixture by Geoffroy ${ }^{10}$ and selectively (at low temperature) as the $E$-isomer by Bickelhaupt ${ }^{22}$ using phospha-Peterson methodology. ${ }^{17}$ Its full spectroscopic characterization was reported in Ref 22. Using phospha-Wittig methodology, in a manner as described below for $\mathbf{2 b}$, parent $\mathbf{2 a}$ was isolated in $83 \%$ yield.

Reference 10,17, and 56 can be found in the manuscript. 


\section{Synthesis of $2 \mathbf{b}(\mathrm{R}=\mathrm{Me})$}

Mes* $\mathrm{PCl}_{2}(1.00 \mathrm{~g}, 2.88 \mathrm{mmol})$ and $\mathrm{Zn}$ dust $(940 \mathrm{mg}, 14.4 \mathrm{mmol}, 5$ equiv.) were combined in a vial, and $4 \mathrm{~mL}$ of THF was added. Treatment of the suspension with a room temperature solution of $\mathrm{PMe}_{3}$ (560 mg, $7.36 \mathrm{mmol}, 2.6$ equiv.) in THF resulted in a yellow reaction mixture. The reaction mixture was stirred at room temperature for $1 \mathrm{~h}$ and filtered through a Celite plug directly into a solution of 6-methylpyridine-2-carboxaldehyde (262 mg, $2.16 \mathrm{mmol}, 0.75$ equiv.) in $3 \mathrm{~mL}$ of THF to afford a homogeneous yellow-orange solution. After stirring for $10 \mathrm{~min}$, the reaction mixture was concentrated under vacuum. The yellow residue was extracted with $5 \mathrm{~mL}$ of pentane and filtered through a Celite plug. The filtrate was concentrated under vacuum and recrystallized from $5 \mathrm{~mL}$ of a 50:50 solution of THF and acetonitrile at $-35{ }^{\circ} \mathrm{C}$ to give a yellow solid (700 mg, $1.84 \mathrm{mmol}, 85 \%$ yield).

Anal. Calcd. for $\mathrm{C}_{25} \mathrm{H}_{36} \mathrm{NP}$ : C, 78.70; H, 9.51; N, 3.67. Found: C, 78.42; H, 9.54; N, 3.59. HRMS: $m / z$ was calcd for $\mathrm{C}_{25} \mathrm{H}_{36} \mathrm{NP}[\mathrm{M}]^{+}: 381.2585$. Found: $381.2591 .{ }^{31} \mathrm{P}\left\{{ }^{1} \mathrm{H}\right\} \mathrm{NMR}\left(\mathrm{CDCl}_{3}\right)$ : $\delta$ 280.4. ${ }^{1} \mathrm{H}$ NMR $\left(\mathrm{CDCl}_{3}\right): \delta 8.10(\mathrm{~d}, J=25.0 \mathrm{~Hz}, 1 \mathrm{H}, \mathrm{P}=\mathrm{CH}), 7.51(\mathrm{~m}, 2 \mathrm{H}, \mathrm{Ar}), 7.43(\mathrm{~s}, 2 \mathrm{H}$, Ar), 6.97 (br m, 1H, Ar), $2.53(\mathrm{~s}, 3 \mathrm{H}, \mathrm{Me}), 1.52(\mathrm{~s}, 18 \mathrm{H}, t-\mathrm{Bu}), 1.35\left(\mathrm{~s}, 9 \mathrm{H}, t\right.$-Bu). ${ }^{13} \mathrm{C}\left\{{ }^{1} \mathrm{H}\right\} \mathrm{NMR}$ $\left(\mathrm{CDCl}_{3}\right): \delta 175.6(\mathrm{~d}, J=33.8, \mathrm{P}=\mathrm{C}), 158.2(\mathrm{Ar}), 157.5$ (d, $\left.J=15.0 \mathrm{~Hz}, \mathrm{Ar}\right), 153.9$ (Ar), 149.7 (Ar), 148.5 (Ar), 139.0 (d, $J=52.5 \mathrm{~Hz}, \mathrm{Ar}$ ), 136.4 (Ar), 123.7 (Ar), 121.6 (Ar), 119.4 (Ar), 117.0 $(\mathrm{d}, J=20.0 \mathrm{~Hz}, \mathrm{Ar}), 38.2\left(\mathrm{CMe}_{3}\right), 34.0(t-\mathrm{Bu}), 33.9(t-\mathrm{Bu}), 31.8\left(\mathrm{CMe}_{3}\right), 31.6\left(\mathrm{CMe}_{3}\right), 31.4(t-$ $\mathrm{Bu}), 30.9$ (Me). 
${ }^{31} \mathrm{P}\left\{{ }^{1} \mathrm{H}\right\}$ NMR $\left(\mathrm{CDCl}_{3}\right)$

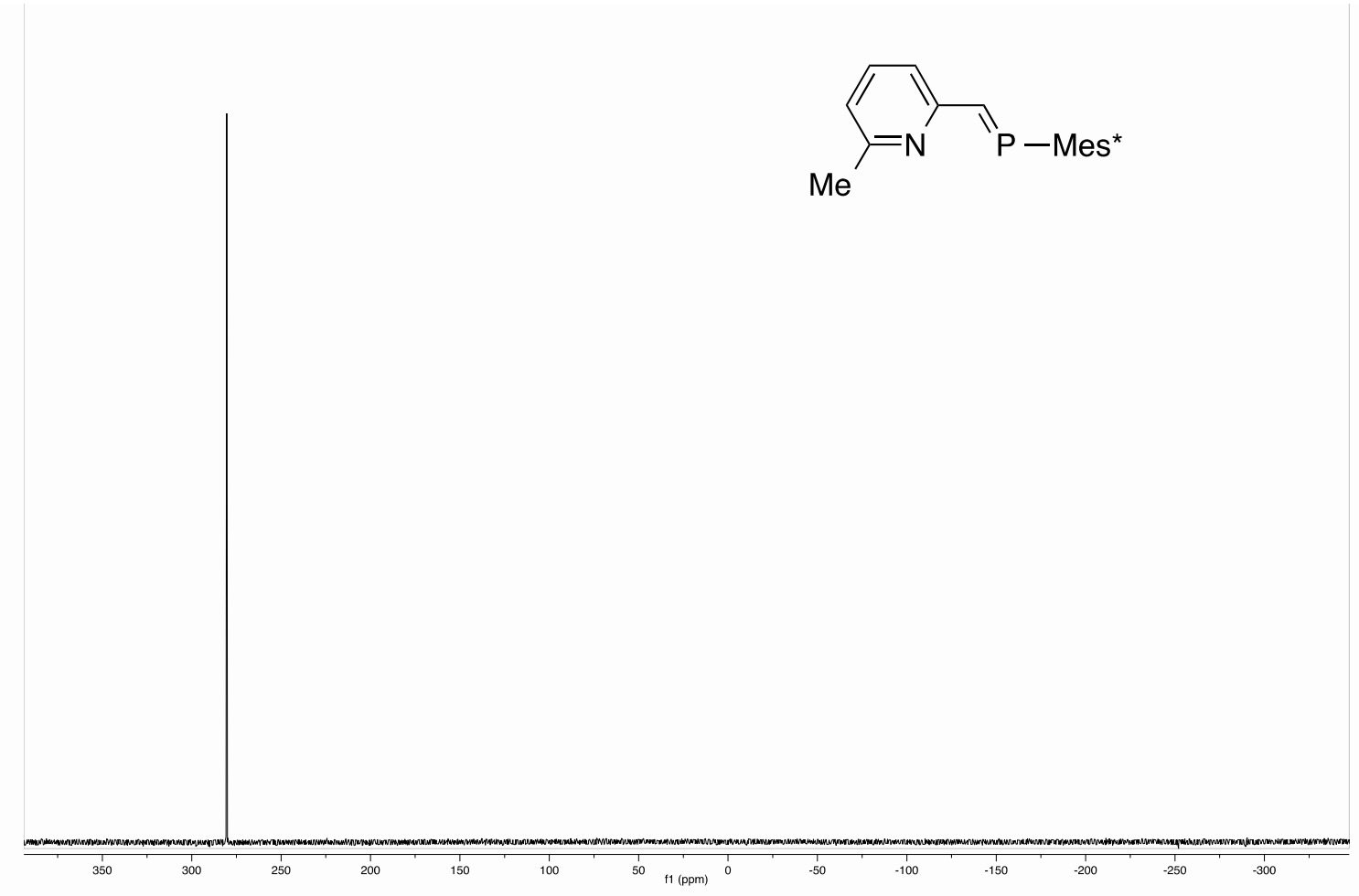

${ }^{1} \mathrm{H} \mathrm{NMR}\left(\mathrm{CDCl}_{3}\right)$

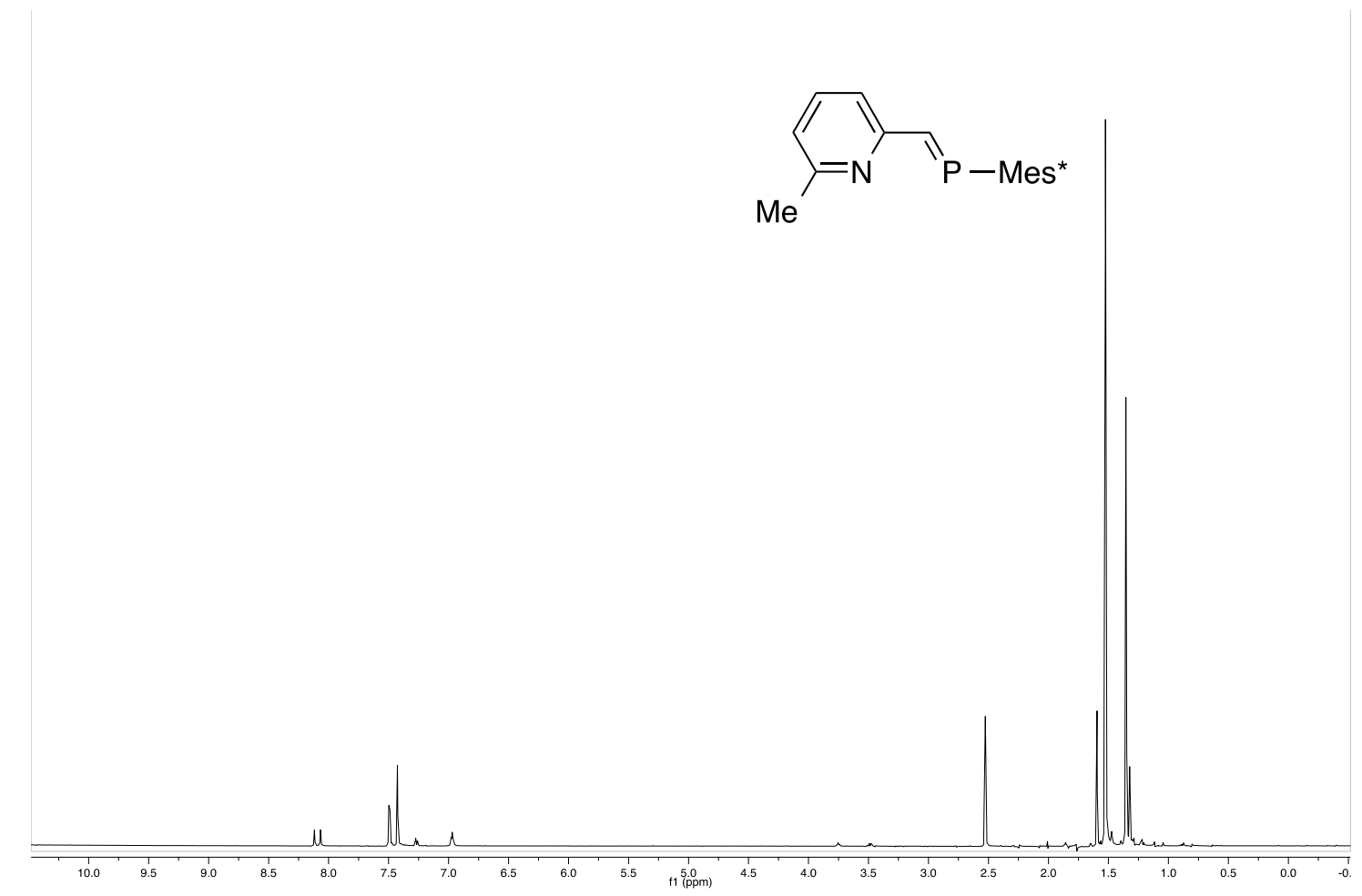


${ }^{1} \mathrm{H}$ NMR $\left(\mathrm{CDCl}_{3}\right)$ : Aryl and Phosphaalkene Region
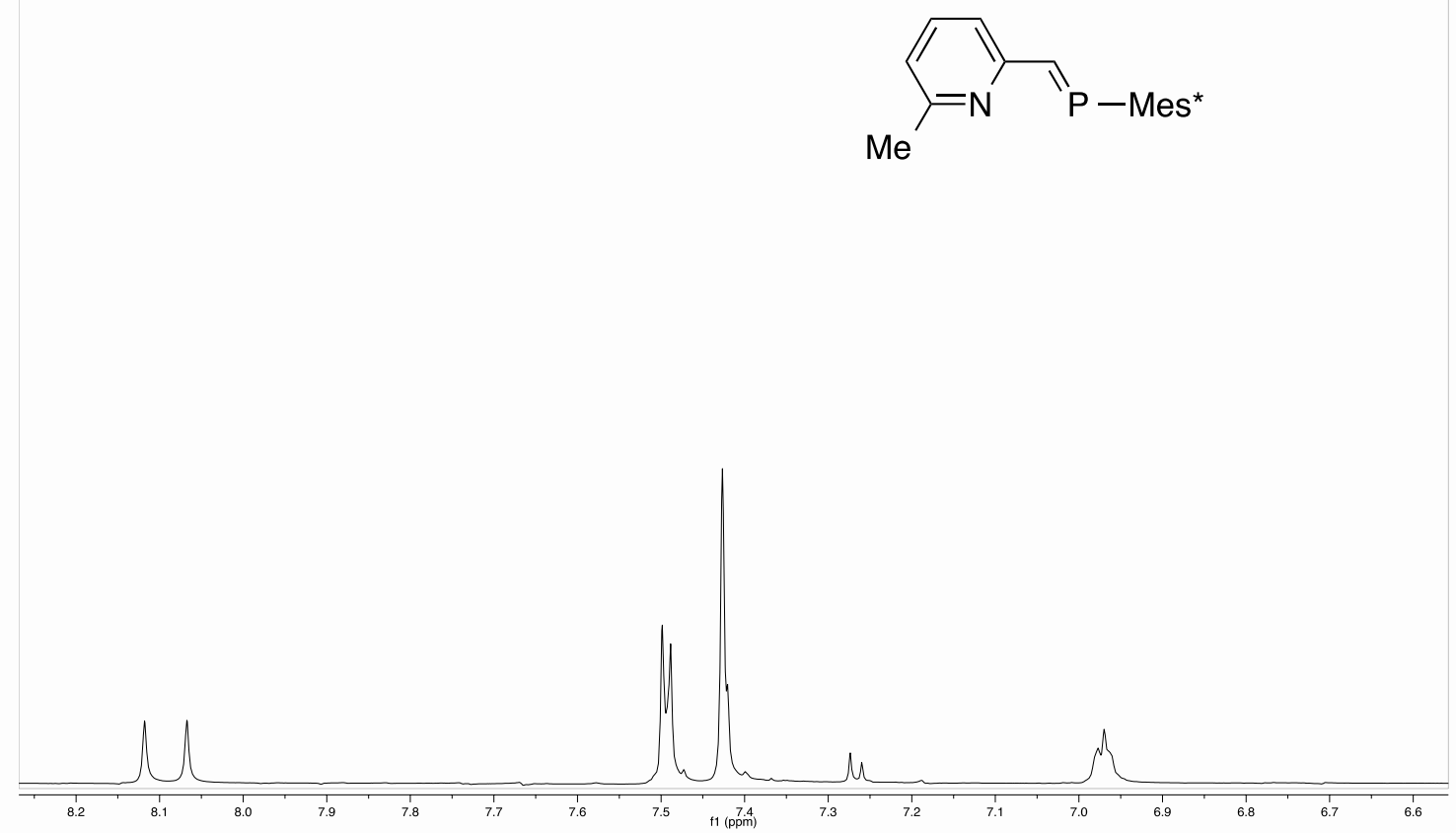

${ }^{13} \mathrm{C}\left\{{ }^{1} \mathrm{H}\right\}$ NMR $\left(\mathrm{CDCl}_{3}\right)$

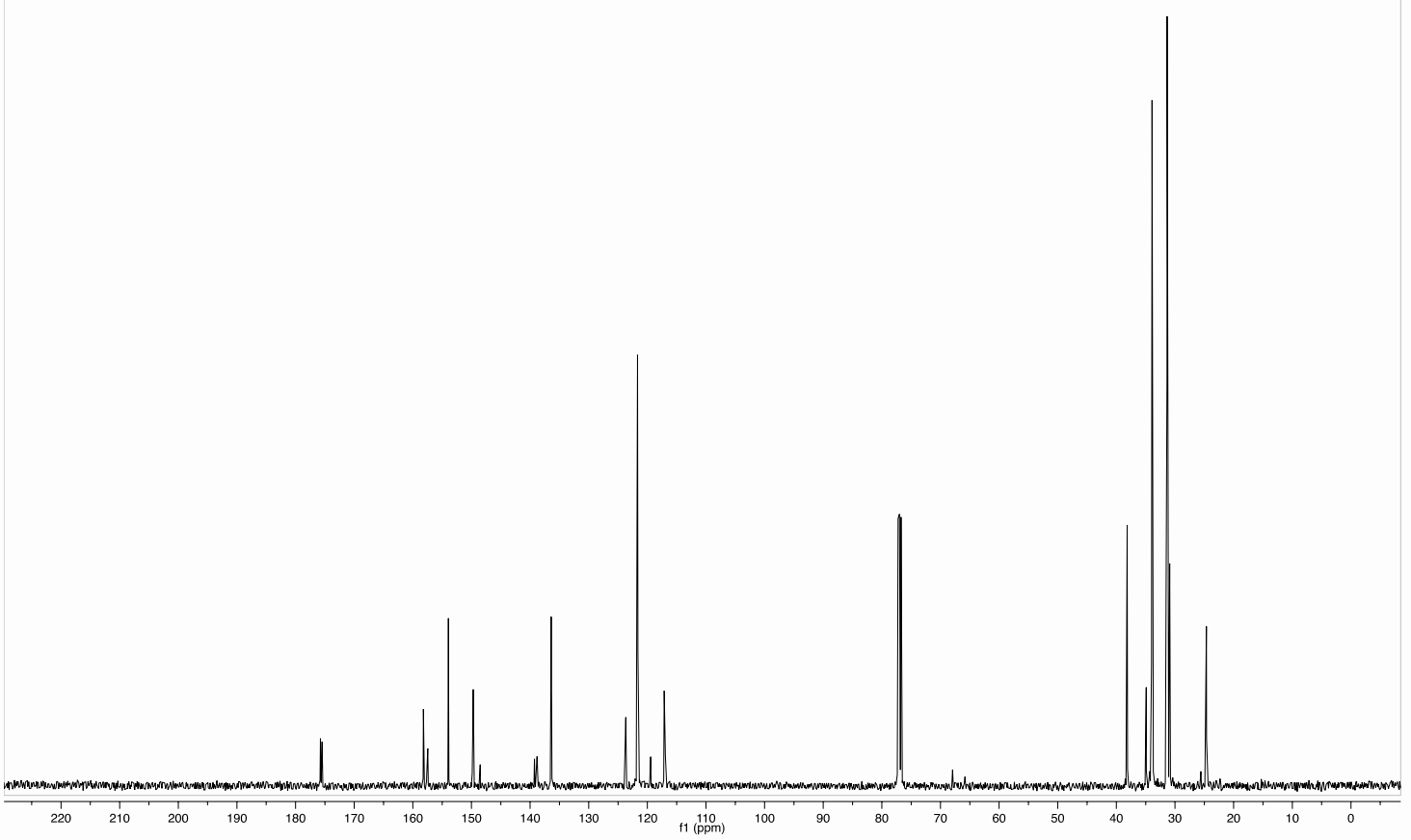




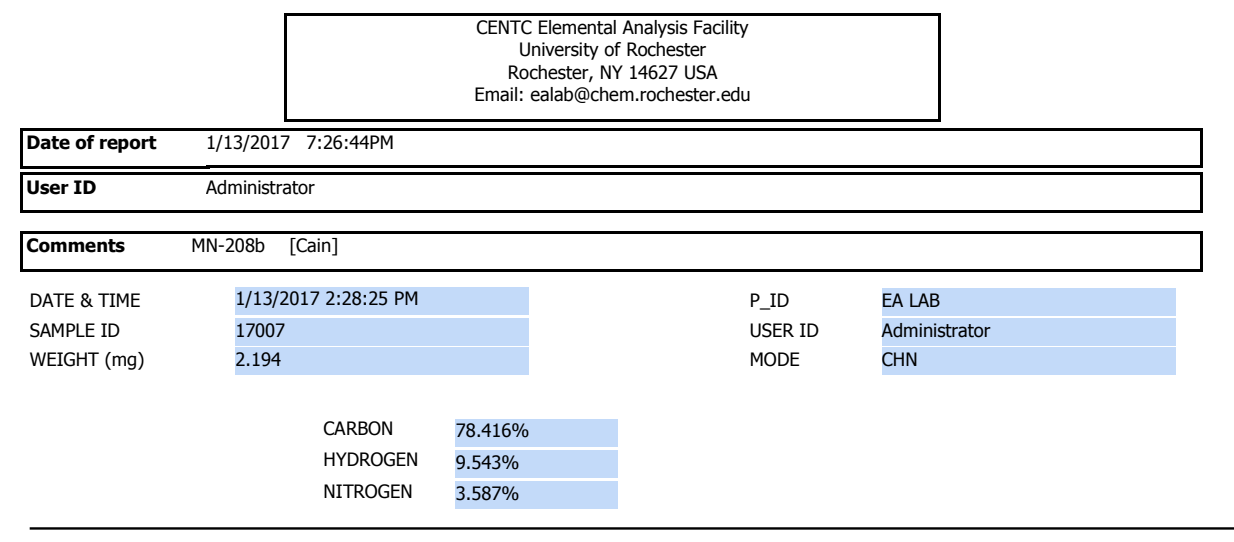

\section{Acknowledgment}

Analytical data were obtained from the CENTC Elemental Analysis Facility at the University of Rochester, funded by NSF CHE-0650456.

\section{$\underline{\text { Instrumentation }}$}

Microanalysis samples were weighed with a PerkinElmer Model AD-6 Autobalance and their compositions were determined with a PerkinElmer 2400 Series II Analyzer.

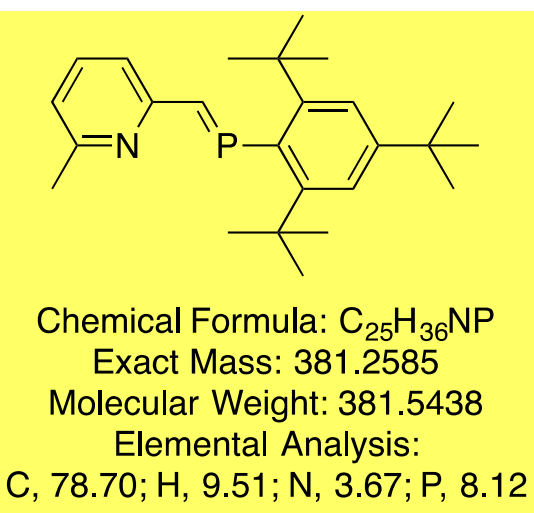

Page 1 of 1 


\section{Synthesis of $2 \mathrm{c}\left(\mathrm{R}=\mathrm{CF}_{3}\right)$}

$\mathrm{Mes}^{*} \mathrm{PCl}_{2}(1.00 \mathrm{~g}, 2.88 \mathrm{mmol})$ and $\mathrm{Zn}$ dust $(940 \mathrm{mg}, 14.4 \mathrm{mmol}, 5$ equiv.) were combined in a vial, and $4 \mathrm{~mL}$ of benzene was added. The heterogeneous mixture was cooled to $-35{ }^{\circ} \mathrm{C}$ in the glove box freezer for $5 \mathrm{~min}$. Treatment of the frozen benzene mixture with a room temperature solution of $\mathrm{PMe}_{3}(560 \mathrm{mg}, 7.36 \mathrm{mmol}, 2.6$ equiv.) in benzene resulted in a yellow reaction mixture. The reaction mixture was stirred at room temperature for $1 \mathrm{~h}$ and filtered through a Celite plug directly into a solution of 6-trifluoromethylpyridine-2-carboxaldehyde (378 mg, 2.16 mmol, 0.75 equiv.) in $3 \mathrm{~mL}$ of benzene to afford a homogeneous yellow-orange solution. After stirring for $10 \mathrm{~min}$, the reaction mixture was concentrated under vacuum. The yellow residue was washed with $5 \mathrm{~mL}$ of pentane and filtered through a Celite plug. The filtrate was concentrated under vacuum and recrystallized from $5 \mathrm{~mL}$ of a 50:50 solution of THF and acetonitrile at -35 ${ }^{\circ} \mathrm{C}$ (495 mg, $1.15 \mathrm{mmol}, 53 \%$ yield). The ligand was not stable long-term in the solid-state, even in the glove box. It was isolated, then immediately used in the synthesis of $\mathbf{6 c}$.

HRMS: $m / z$ was calcd for $\mathrm{C}_{25} \mathrm{H}_{34} \mathrm{~F}_{3} \mathrm{NP}(\mathrm{M}+\mathrm{H})^{+}$: 436.2381. Found: 436.2383. ${ }^{31} \mathrm{P}\left\{{ }^{1} \mathrm{H}\right\} \mathrm{NMR}$ $\left(\mathrm{CDCl}_{3}\right): \delta 295.5 .{ }^{19} \mathrm{~F}\left\{{ }^{1} \mathrm{H}\right\} \mathrm{NMR}\left(\mathrm{CDCl}_{3}\right): \delta-68.8 .{ }^{1} \mathrm{H} \mathrm{NMR}\left(\mathrm{CDCl}_{3}\right): \delta 8.15(\mathrm{~d}, J=25 \mathrm{~Hz}, 1 \mathrm{H}$, $\mathrm{P}=\mathrm{CH}), 7.98(\mathrm{~d}, J=10 \mathrm{~Hz}, 1 \mathrm{H}, \mathrm{Ar}), 7.77(\mathrm{t}, J=10 \mathrm{~Hz}, 1 \mathrm{H}, \mathrm{Ar}), 7.48(\mathrm{~d}, J=5 \mathrm{~Hz}, 1 \mathrm{H}, \mathrm{Ar}), 7.44$ (s, 1H, Mes*), $7.42\left(\mathrm{~s}, 1 \mathrm{H}, \mathrm{Mes}^{*}\right), 1.60(\mathrm{~s}, 9 \mathrm{H}, t$-Bu), 1.52 (s, 9H, $t$-Bu), 1.37 (s, 9H, $t$-Bu). ${ }^{13} \mathrm{C}\left\{{ }^{1} \mathrm{H}\right\}$ NMR $\left(\mathrm{CDCl}_{3}\right): \delta 173.1(\mathrm{~d}, J=35.7 \mathrm{~Hz}, \mathrm{P}=\mathrm{C}), 158.7(\mathrm{~d}, J=18.3 \mathrm{~Hz}, \mathrm{Ar}), 153.9$ (Ar), 150.1 (Ar), 148.5 (Ar), 137.4 (Ar), 123.7 (Ar), 121.9 (Ar), 119.5 (Ar), 118.2 (Ar), $38.3\left(C \mathrm{Me}_{3}\right)$, $38.2\left(C \mathrm{Me}_{3}\right), 35.0\left(C \mathrm{Me}_{3}\right), 34.0(\mathrm{~d}, J=6.6 \mathrm{~Hz}, t-\mathrm{Bu}), 31.6(t-\mathrm{Bu}), 31.3(t-\mathrm{Bu}), 30.9(t-\mathrm{Bu})$. 
${ }^{31} \mathrm{P}\left\{{ }^{1} \mathrm{H}\right\} \mathrm{NMR}\left(\mathrm{CDCl}_{3}\right)$

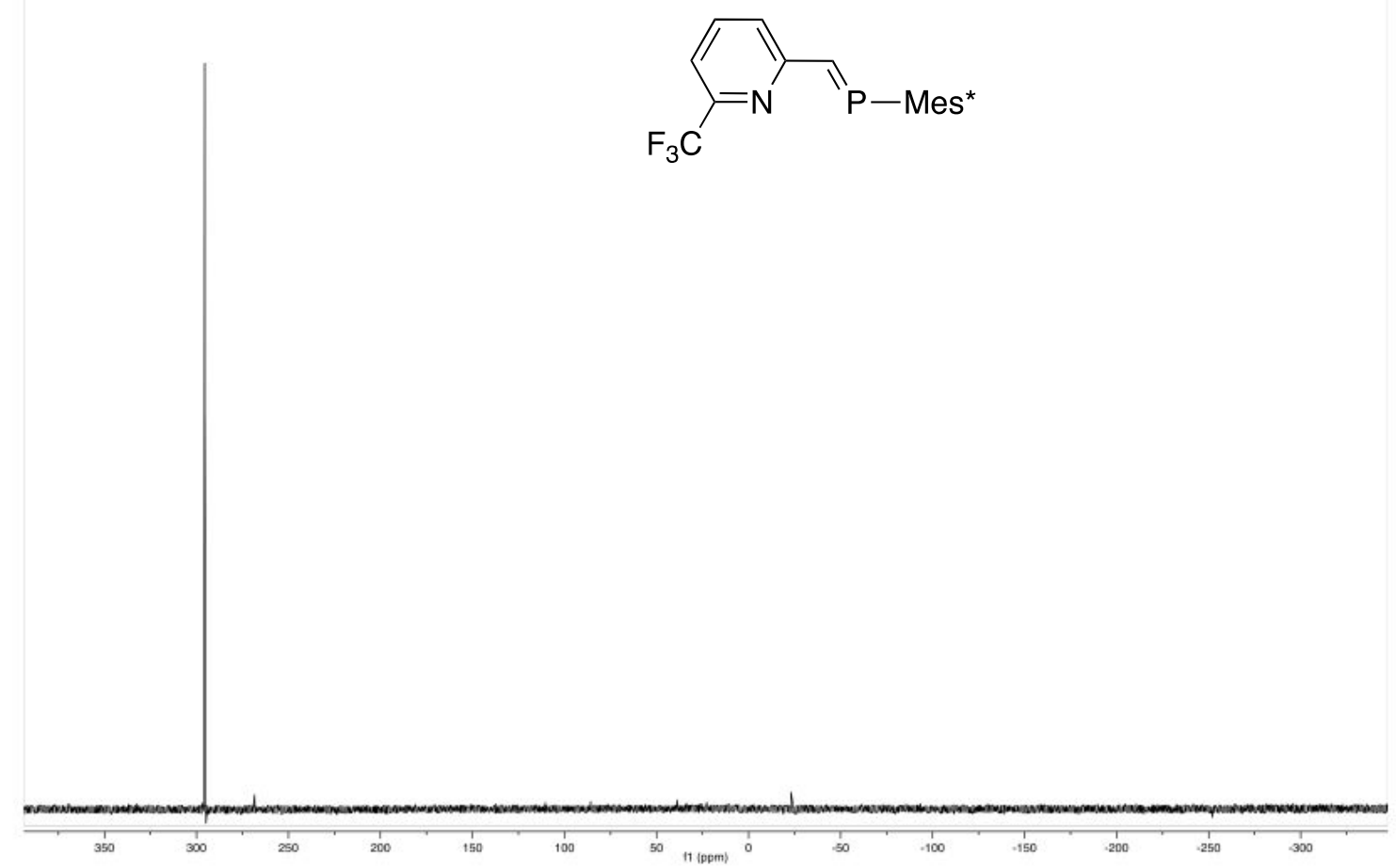

${ }^{1} \mathrm{H} \mathrm{NMR}\left(\mathrm{CDCl}_{3}\right)$

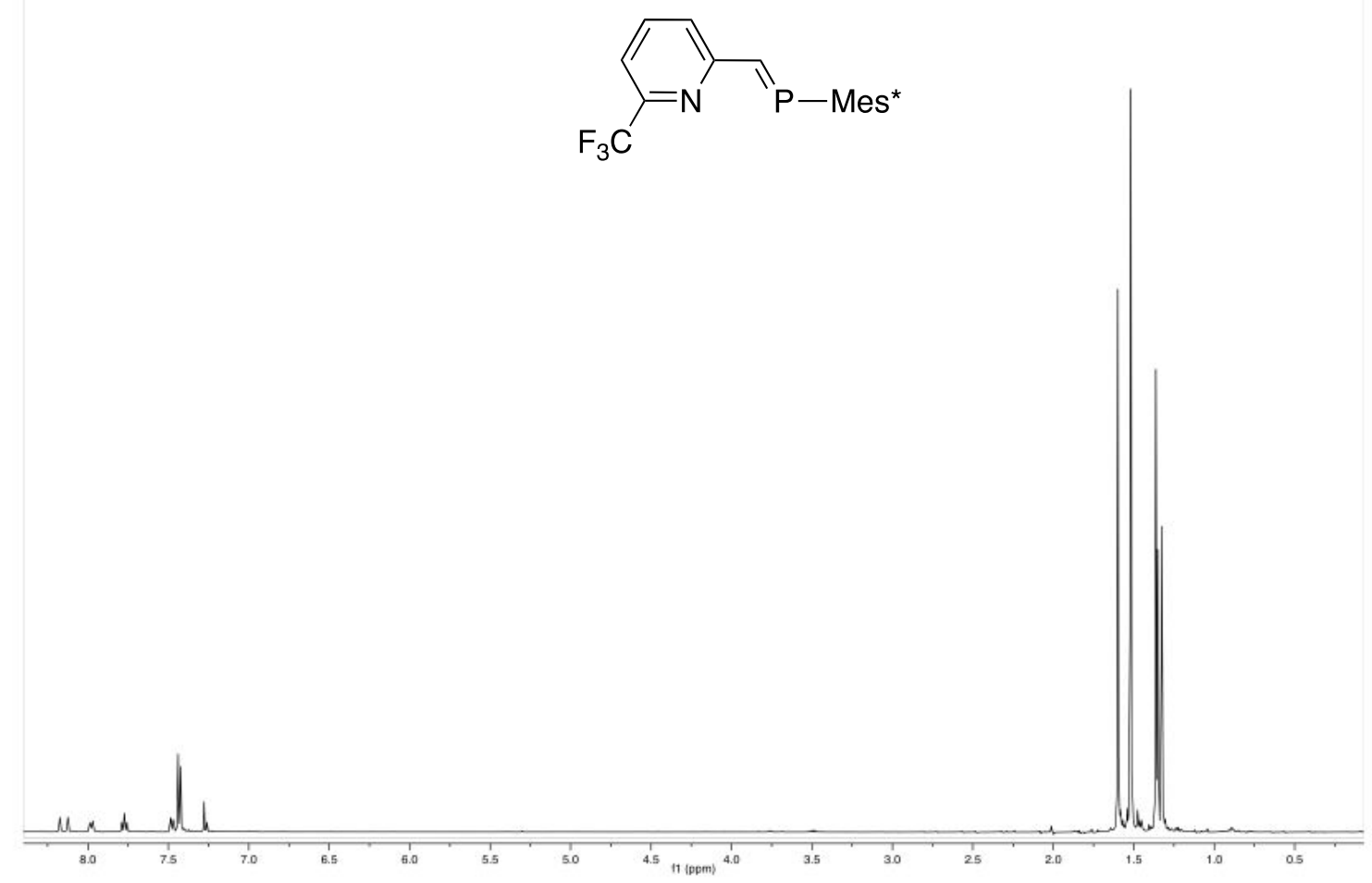


${ }^{13} \mathrm{C}\left\{{ }^{1} \mathrm{H}\right\}$ NMR $\left(\mathrm{CDCl}_{3}\right)$

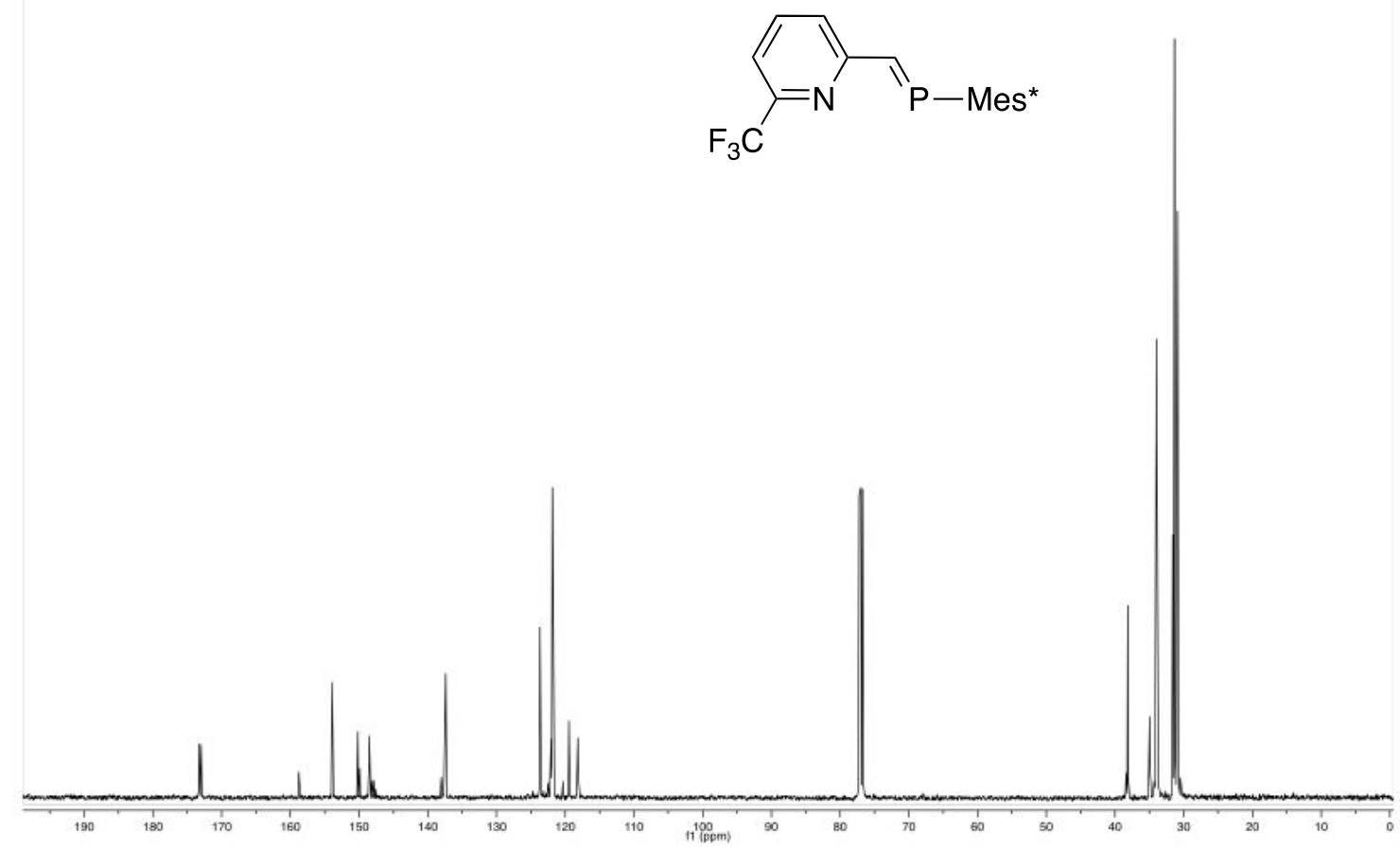

${ }^{19} \mathrm{~F}$ NMR $\left(\mathrm{CDCl}_{3}\right)$

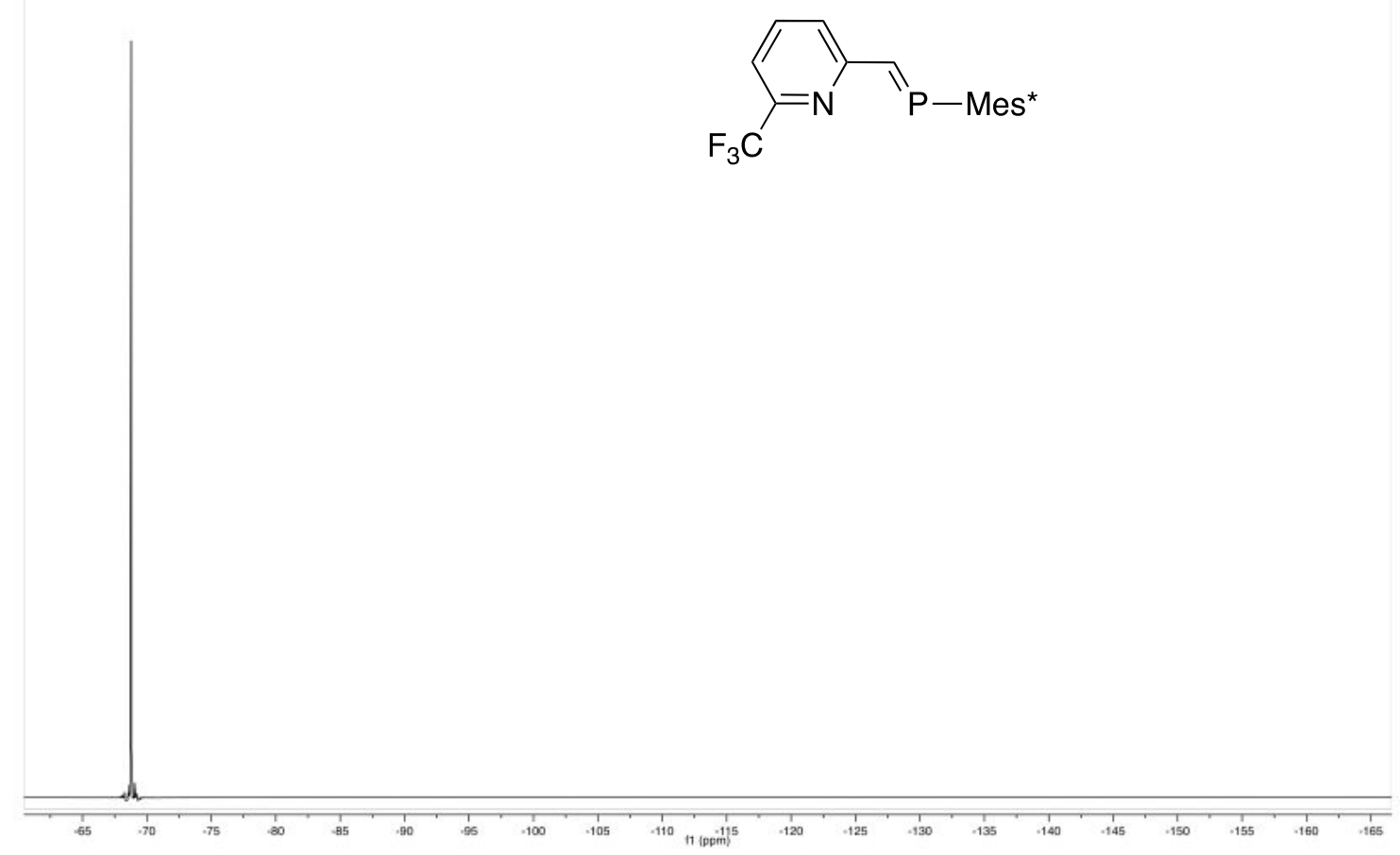




\section{Synthesis of $2 d(\mathrm{R}=\mathrm{OMe})$}

$\mathrm{Mes}^{*} \mathrm{PCl}_{2}$ (300 mg, $\left.0.960 \mathrm{mmol}\right)$ and $\mathrm{Zn}$ dust (290 mg, $4.58 \mathrm{mmol}, 5$ equiv.) were combined in a vial, and $4 \mathrm{~mL}$ of benzene was added. The heterogeneous mixture was cooled in the glove box freezer $\left(-35^{\circ} \mathrm{C}\right)$ for $5 \mathrm{~min}$. Treatment of the frozen benzene mixture with a room temperature solution of $\mathrm{PMe}_{3}$ (186 mg, $2.45 \mathrm{mmol}, 2.6$ equiv.) in benzene resulted in a yellow reaction mixture. The reaction mixture was stirred at room temperature for $1 \mathrm{~h}$ and filtered through a Celite plug directly into a solution of 6-methoxypyridine-2-carboxaldehyde (99 $\mathrm{mg}, 0.72 \mathrm{mmol}$, 0.75 equiv.) in $3 \mathrm{~mL}$ of benzene to afford a homogeneous yellow-orange solution. After stirring for $10 \mathrm{~min}$, the reaction mixture was concentrated under vacuum. The yellow residue was extracted with $5 \mathrm{~mL}$ of pentane and filtered through a Celite plug. The filtrate was concentrated under vacuum and recrystallized from $5 \mathrm{~mL}$ of a 50:50 solution of THF and acetonitrile at -35 ${ }^{\circ} \mathrm{C}$ to give a yellow solid (214 $\mathrm{mg}, 0.54 \mathrm{mmol}, 75 \%$ yield).

Anal. Calcd. for $\mathrm{C}_{25} \mathrm{H}_{36} \mathrm{NOP}$ : C, 75.53; H, 9.13; N, 3.52. Found: C, 75.19; H, 9.30; N, 3.47. HRMS: $m / z$ was calcd for $\mathrm{C}_{25} \mathrm{H}_{36} \mathrm{NOP}[\mathrm{M}]^{+}:$397.2535. Found: 397.2540. ${ }^{31} \mathrm{P}\left\{{ }^{1} \mathrm{H}\right\} \mathrm{NMR}$ $\left(\mathrm{CDCl}_{3}\right): \delta 281.0 .{ }^{1} \mathrm{H} \mathrm{NMR}\left(\mathrm{CDCl}_{3}\right): \delta 8.01(\mathrm{~d}, J=25.0 \mathrm{~Hz}, 1 \mathrm{H}, \mathrm{P}=\mathrm{CH}), 7.47(\mathrm{t}, J=8.0 \mathrm{~Hz}, 1 \mathrm{H}$, Ar), 7.44 (s, 2H, Mes*), 6.87 (d, J=7.0 Hz, 1H, Ar), 6.60 (d, J=8.5 Hz, 1H, Ar), 3.98 (s, 3H, $\mathrm{OMe}), 1.53\left(\mathrm{~s}, 18 \mathrm{H}, t\right.$-Bu), $1.36\left(\mathrm{~s}, 9 \mathrm{H}, t\right.$-Bu). ${ }^{13} \mathrm{C}\left\{{ }^{1} \mathrm{H}\right\} \mathrm{NMR}\left(\mathrm{CDCl}_{3}\right): \delta 173.8(\mathrm{~d}, J=33.8 \mathrm{~Hz}$, $\mathrm{P}=\mathrm{C}$ ), 163.4 (Ar), 155.7 (Ar), 153.9 (Ar), 149.6 (Ar), 139.9 (Ar), 138.7 (Ar), 121.7 (Ar). 113.9 (d, $J=18.8 \mathrm{~Hz}, \mathrm{Ar}), 109.5(\mathrm{Ar}), 53.3(\mathrm{MeO}), 38.3\left(\mathrm{CMe}_{3}\right), 35.0\left(\mathrm{CMe}_{3}\right), 33.9$ (d, $J=7.5 \mathrm{~Hz}, t$ $\mathrm{Bu}), 31.4(t-\mathrm{Bu})$. 
${ }^{31} \mathrm{P}\left\{{ }^{1} \mathrm{H}\right\} \mathrm{NMR}\left(\mathrm{CDCl}_{3}\right)$
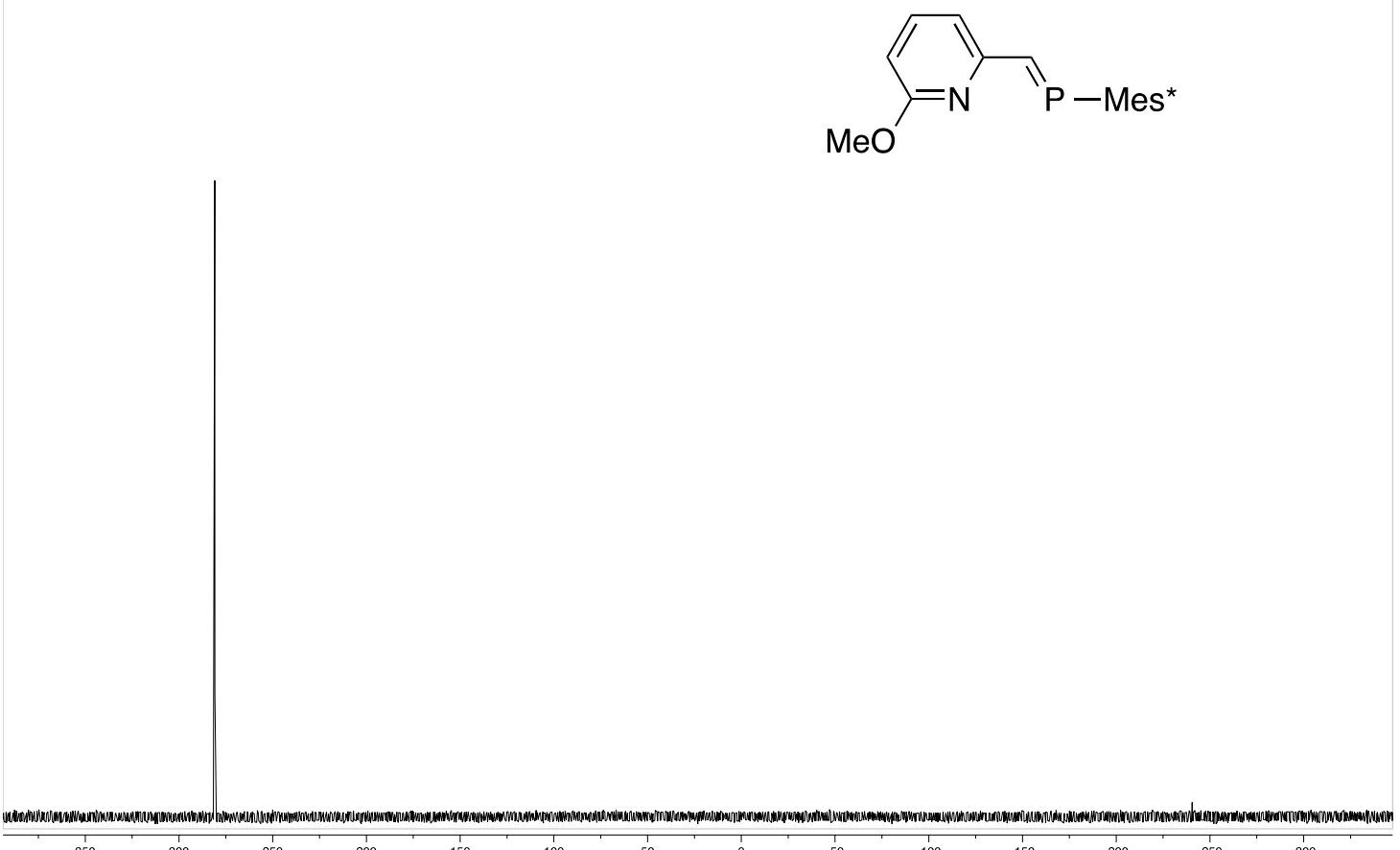

${ }^{1} \mathrm{H} \mathrm{NMR}\left(\mathrm{CDCl}_{3}\right)$

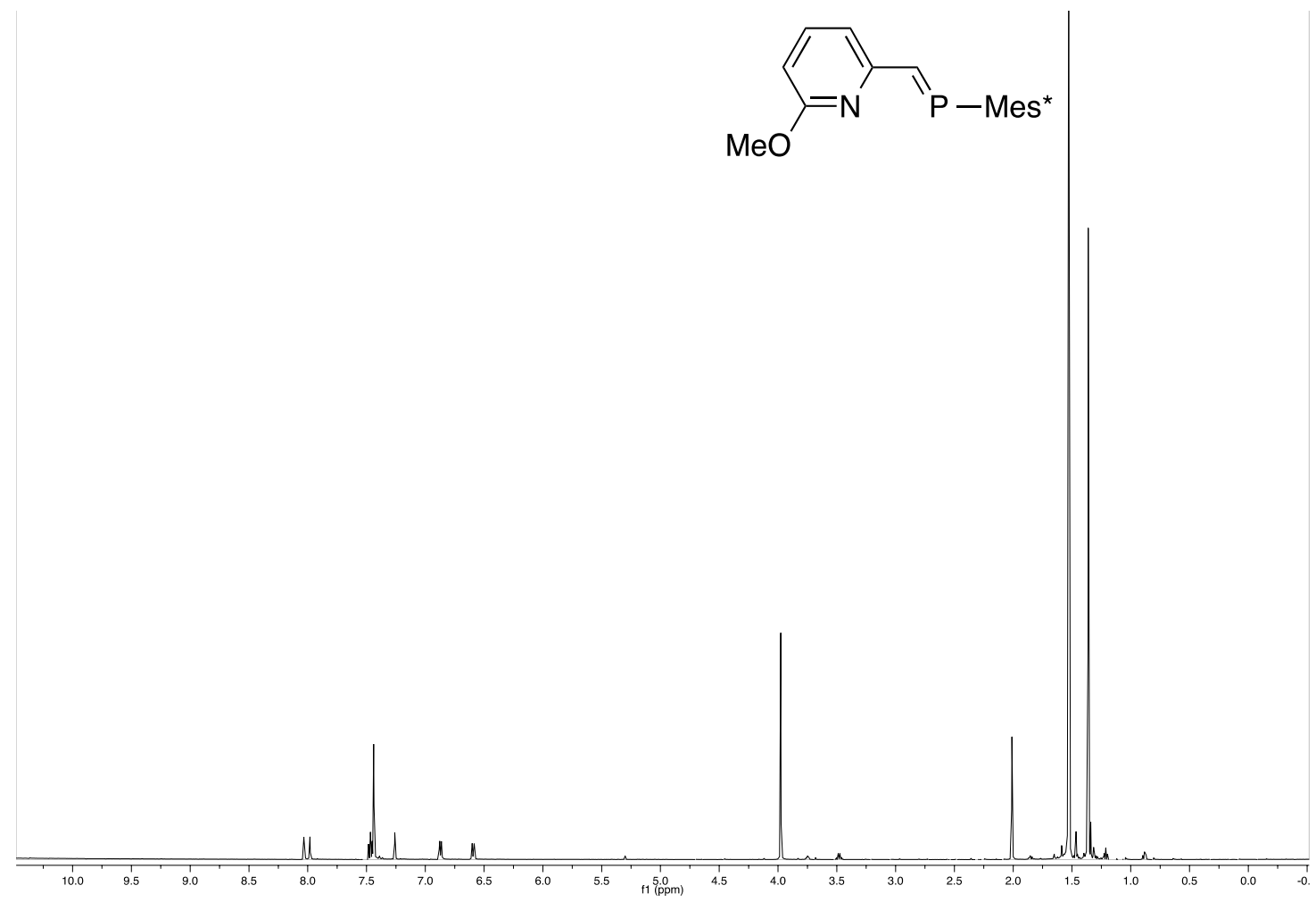


${ }^{1} \mathrm{H}$ NMR $\left(\mathrm{CDCl}_{3}\right)$ : Aryl and Phosphaalkene Region

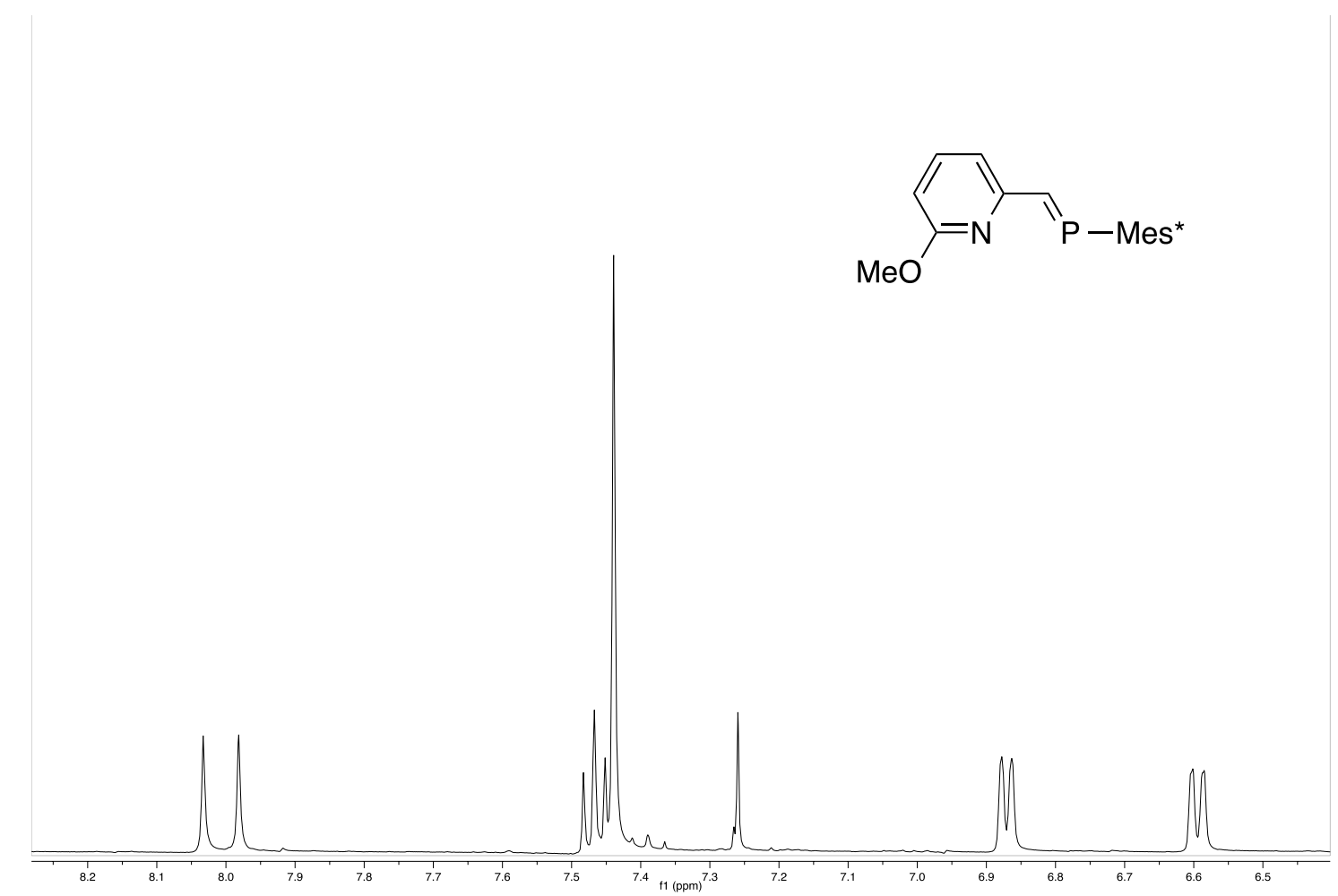

${ }^{13} \mathrm{C}\left\{{ }^{1} \mathrm{H}\right\}$ NMR $\left(\mathrm{CDCl}_{3}\right)$

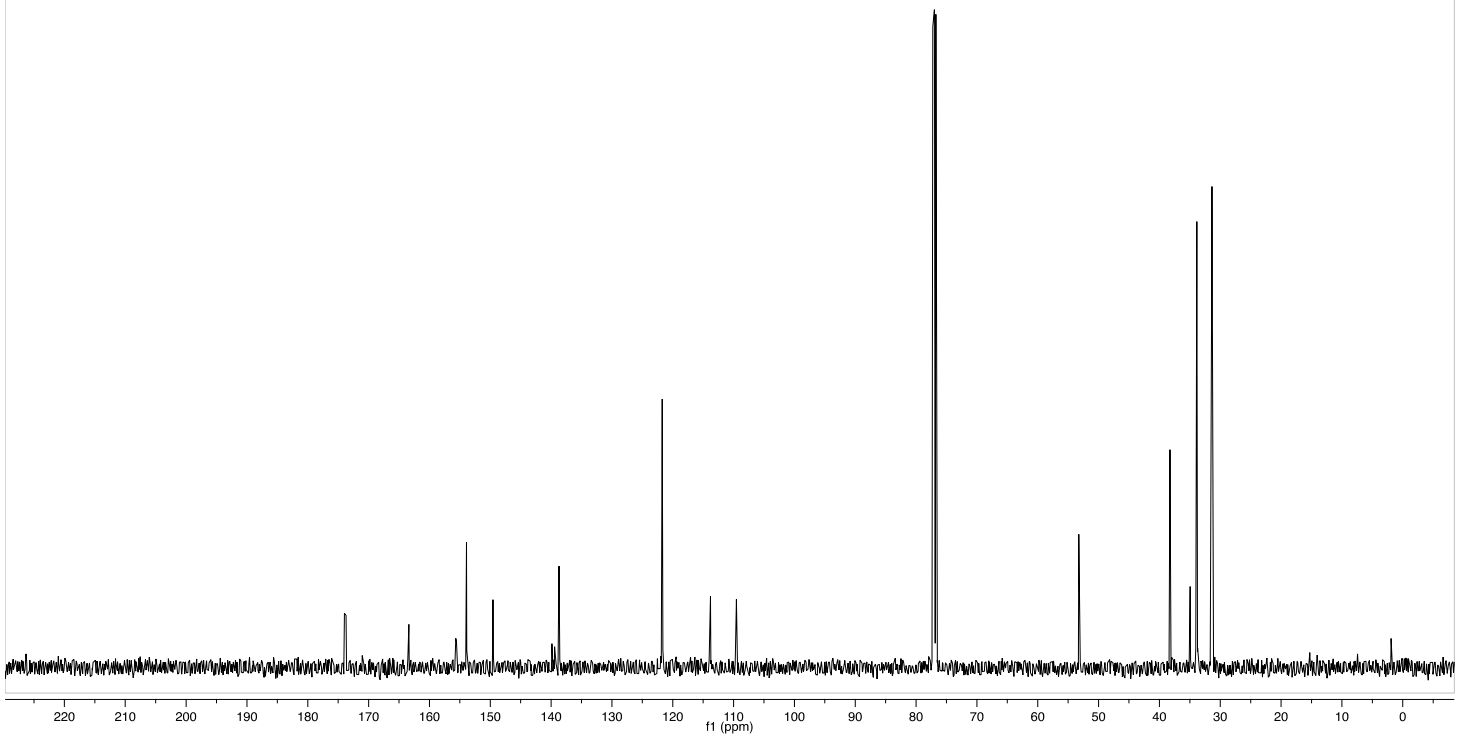




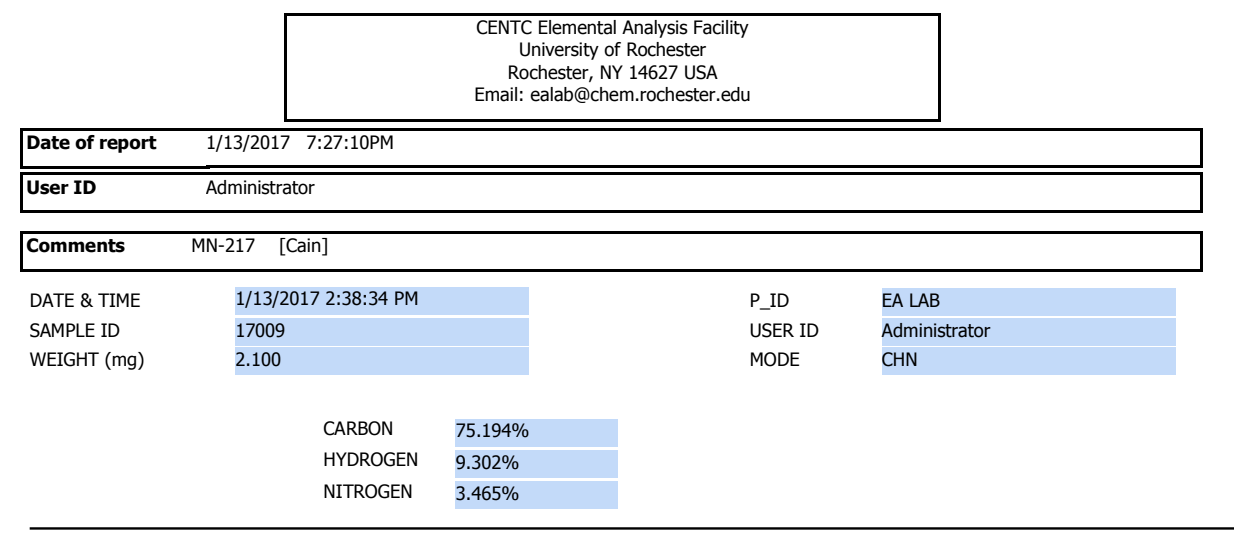

\section{Acknowledgment}

Analytical data were obtained from the CENTC Elemental Analysis Facility at the University of Rochester, funded by NSF CHE-0650456.

\section{$\underline{\text { Instrumentation }}$}

Microanalysis samples were weighed with a PerkinElmer Model AD-6 Autobalance and their compositions were determined with a PerkinElmer 2400 Series II Analyzer.

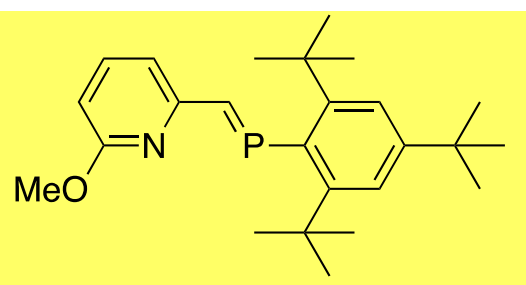

Chemical Formula: $\mathrm{C}_{25} \mathrm{H}_{36} \mathrm{NOP}$

Exact Mass: 397.2535

Molecular Weight: 397.5428

Elemental Analysis:

C, 75.53; H, 9.13; N, 3.52; O, 4.02; P, 7.79

Page 1 of 1 
Synthesis of $2 \mathbf{e}\left(\mathrm{R}=\mathrm{Ar}-m-\mathrm{NO}_{2}\right)$

Mes* $\mathrm{PCl}_{2}(1.00 \mathrm{~g}, 2.88 \mathrm{mmol})$ and $\mathrm{Zn}$ dust $(940 \mathrm{mg}, 14.4 \mathrm{mmol}, 5$ equiv.) were combined in a vial, and $4 \mathrm{~mL}$ of benzene was added. The heterogeneous mixture was cooled to $-35^{\circ} \mathrm{C}$ in the glove box freezer for $5 \mathrm{~min}$. Treatment of the frozen benzene mixture with a room temperature solution of $\mathrm{PMe}_{3}(560 \mathrm{mg}, 7.36 \mathrm{mmol}, 2.6$ equiv.) resulted in a yellow reaction mixture. The reaction mixture was stirred at room temperature for $1 \mathrm{~h}$ and filtered through a Celite plug directly into a solution of 6-(3-nitrophenyl)-2-pyridinecarboxaldehyde (493 mg, $2.16 \mathrm{mmol}, 0.75$ equiv.) in $3 \mathrm{~mL}$ of benzene to afford a homogeneous yellow-orange solution. After stirring for 10 min, the reaction mixture was concentrated under vacuum. The yellow residue was extracted with $5 \mathrm{~mL}$ of pentane and filtered through a Celite plug. The filtrate was concentrated under vacuum and recrystallized from $5 \mathrm{~mL}$ of a 50:50 solution of THF and acetonitrile at $-35{ }^{\circ} \mathrm{C}$ to give a yellow solid $(760 \mathrm{mg}, 1.56 \mathrm{mmol}, 72 \%$ yield). Crystals suitable X-ray diffraction were obtained from this initial recrystallization.

Elemental Analysis for $\mathrm{C}_{30} \mathrm{H}_{37} \mathrm{~N}_{2} \mathrm{O}_{2} \mathrm{P}$ was consistently low (3 attempts) in carbon, for example: $\mathrm{C}_{30} \mathrm{H}_{38} \mathrm{~N}_{2} \mathrm{O}_{2} \mathrm{P}: \mathrm{C}, 73.75 ; \mathrm{H}, 7.63 ; \mathrm{N}, 5.73$. Found: $\mathrm{C}, 73.01 ; \mathrm{H}, 7.87 ; \mathrm{N}, 5.12$. HRMS: $m / z$ was calcd for $\left.\mathrm{C}_{30} \mathrm{H}_{37} \mathrm{~N}_{2} \mathrm{O}_{2} \mathrm{P}[\mathrm{M}]\right]^{+}: 488.2593$. Found: 488.2601. ${ }^{31} \mathrm{P}\left\{{ }^{1} \mathrm{H}\right\} \mathrm{NMR}\left(\mathrm{CDCl}_{3}\right): \delta 288.5 .{ }^{1} \mathrm{H}$ $\operatorname{NMR}\left(\mathrm{CDCl}_{3}\right): \delta 8.88(\mathrm{t}, J=2.0 \mathrm{~Hz}, 1 \mathrm{H}, \mathrm{Ar}), 8.46(\mathrm{dt}, J=7.5,1.0 \mathrm{~Hz}, 1 \mathrm{H}, \mathrm{Ar}), 8.26(\mathrm{ddd}, J=$ 8.0, 2.0, $1.0 \mathrm{~Hz}, 1 \mathrm{H}, \mathrm{Ar}), 8.21(\mathrm{~d}, J=25.5 \mathrm{~Hz}, 1 \mathrm{H}, \mathrm{P}=\mathrm{CH}), 7.75(\mathrm{t}, J=7.5 \mathrm{~Hz}, 1 \mathrm{H}, \mathrm{Ar}), 7.64-$ 7.60 (overlapping m, 3H, Ar), 7.47 (s, 2H, Mes*), $1.56(\mathrm{~s}, 18 \mathrm{H}, t-\mathrm{Bu}), 1.38(\mathrm{~s}, 9 \mathrm{H}, t-\mathrm{Bu})$. ${ }^{13} \mathrm{C}\left\{{ }^{1} \mathrm{H}\right\}$ NMR $\left(\mathrm{CDCl}_{3}\right): \delta 174.0(\mathrm{~d}, J=35.0 \mathrm{~Hz}, \mathrm{P}=\mathrm{C}), 158.3(\mathrm{~d}, J=60.0 \mathrm{~Hz}, \mathrm{Ar}), 154.2(\mathrm{Ar})$, 154.0 (Ar), 149.9 (Ar), 148.7 (Ar), 141.1 (Ar), 138.7 (d, $J=53.8 \mathrm{~Hz}, \mathrm{Ar}), 137.4$ (Ar), 133.0 (Ar), 129.6 (Ar), 123.7 (Ar), 123.4 (Ar), 121.9 (d, $J=12.5 \mathrm{~Hz}, \mathrm{Ar}), 119.7$ (d, $J=18.8 \mathrm{~Hz}, \mathrm{Ar}), 118.7$ (Ar), $38.2\left(\mathrm{CMe}_{3}\right), 35.0\left(\mathrm{CMe}_{3}\right), 33.9(t-\mathrm{Bu}), 31.4(t-\mathrm{Bu})$. 
${ }^{31} \mathrm{P}\left\{{ }^{1} \mathrm{H}\right\} \mathrm{NMR}\left(\mathrm{CDCl}_{3}\right)$

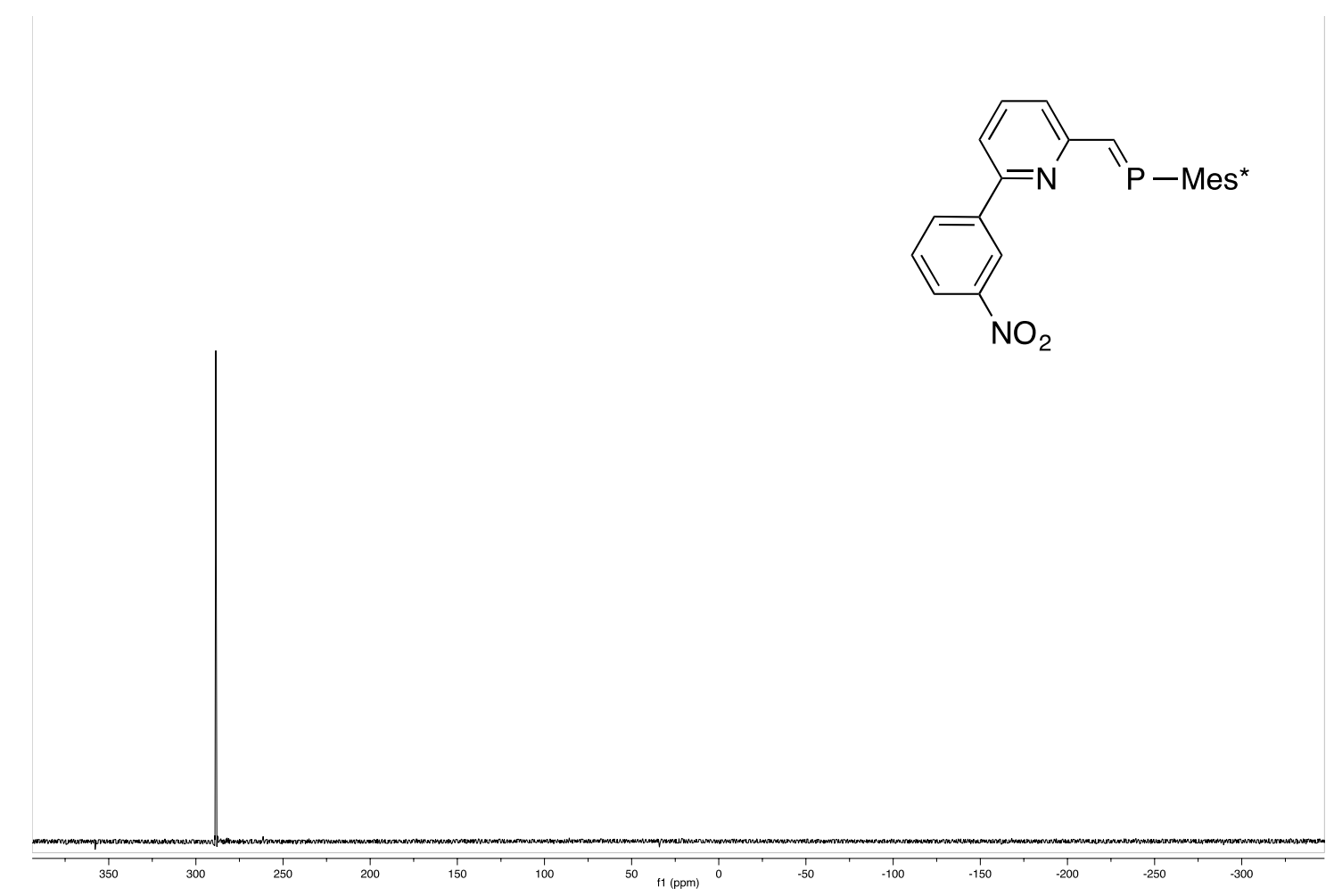

${ }^{1} \mathrm{H} \mathrm{NMR}\left(\mathrm{CDCl}_{3}\right)$

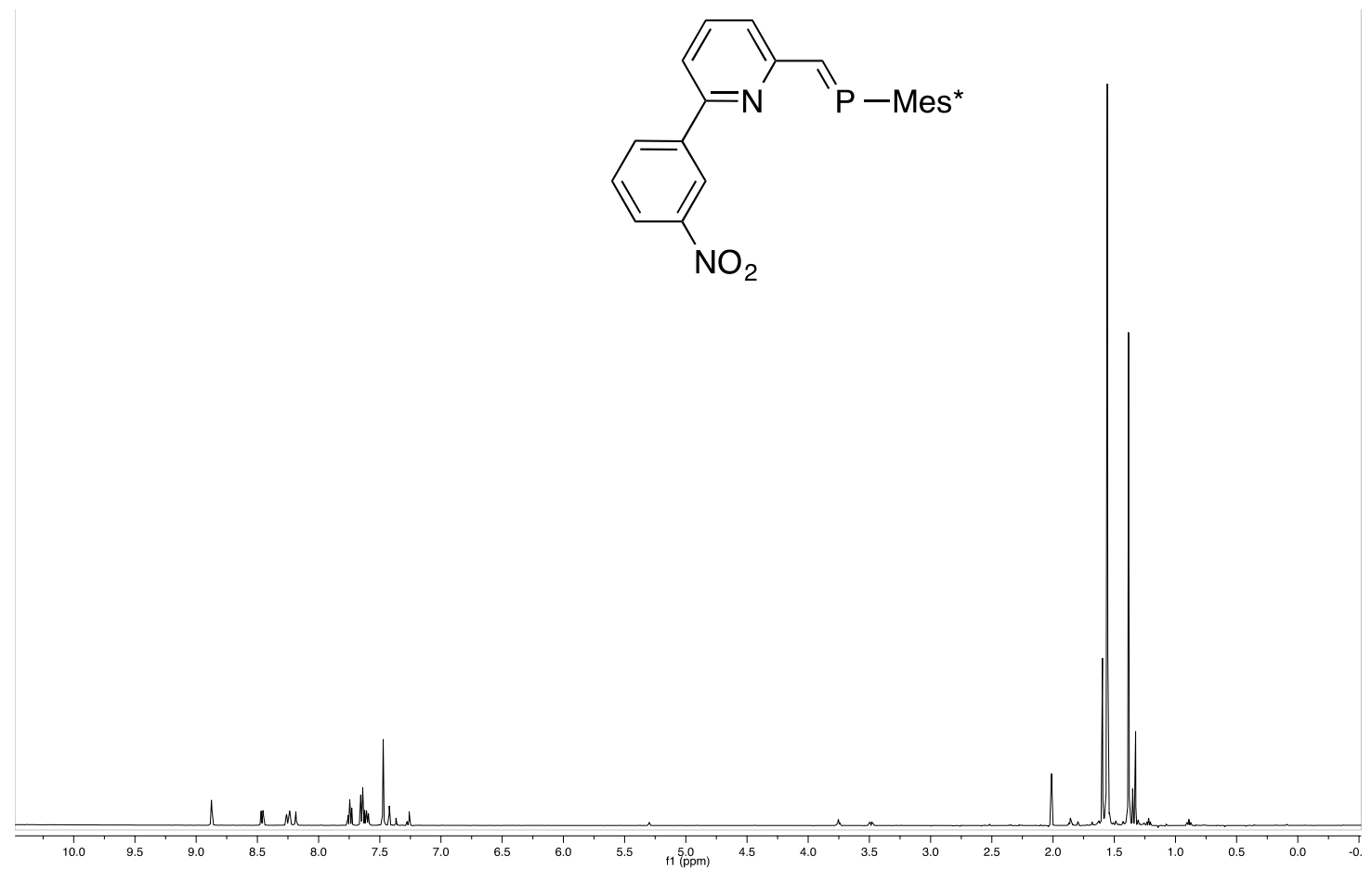


${ }^{1} \mathrm{H}$ NMR $\left(\mathrm{CDCl}_{3}\right)$ : Aryl and Phosphaalkene Region
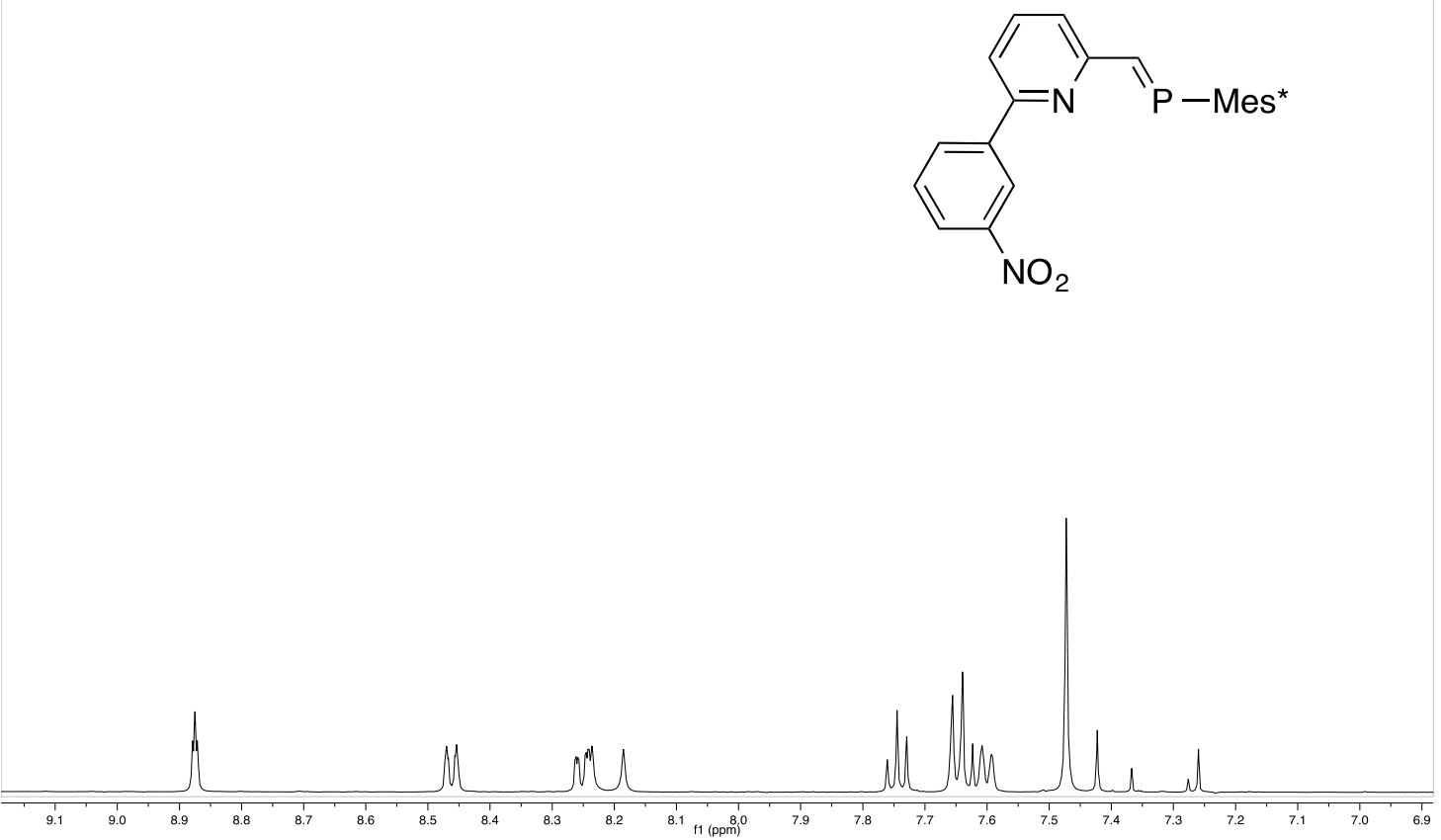

${ }^{13} \mathrm{C}\left\{{ }^{1} \mathrm{H}\right\}$ NMR $\left(\mathrm{CDCl}_{3}\right)$

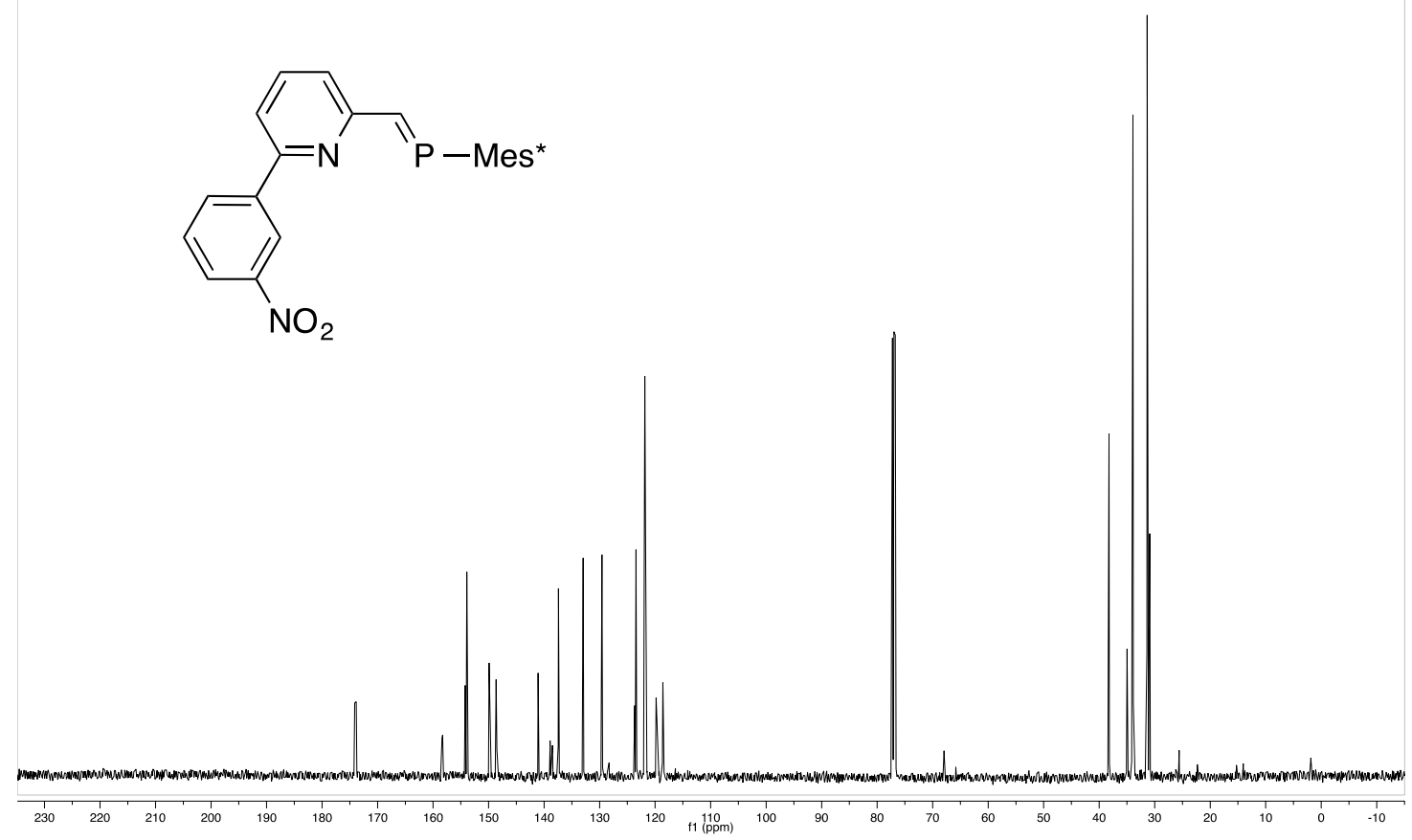




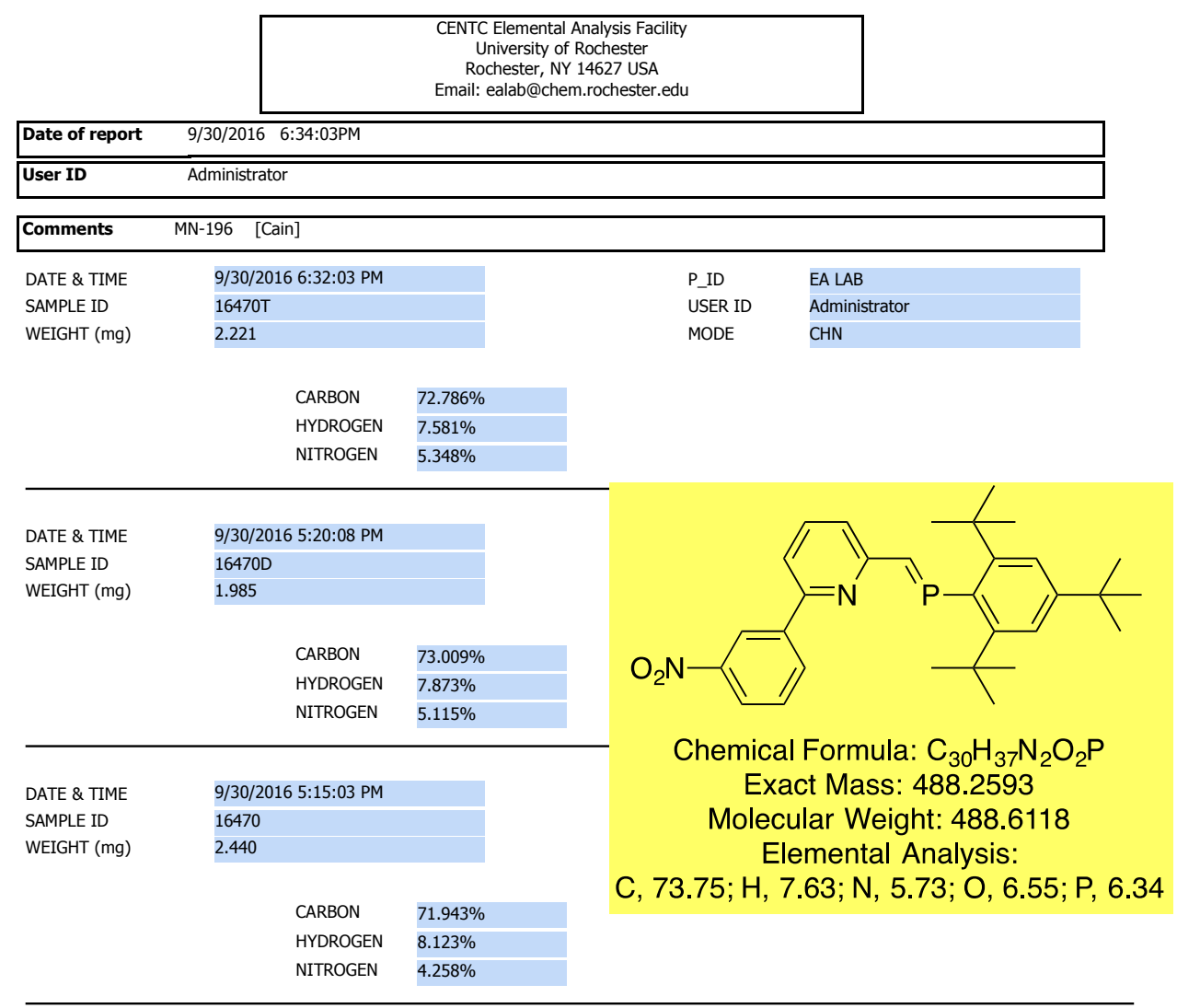

Special Handling

The sample was transferred under argon and was combusted in a tin capsule that was crimp-sealed with a die apparatus.

$\underline{\text { Acknowledgment }}$

Analytical data were obtained from the CENTC Elemental Analysis Facility at the University of Rochester, funded by NSF CHE-0650456.

Instrumentation

Microanalysis samples were weighed with a PerkinElmer Model AD-6 Autobalance and their compositions were determined with a PerkinElmer 2400 Series II Analyzer. Air-sensitive samples were handled in a VAC

Atmospheres glovebox. 


\section{Synthesis of $3 \mathbf{b}(\mathrm{R}=\mathrm{Me})$}

$\mathrm{RuCl}_{2}\left(\mathrm{PPh}_{3}\right)_{3}(126 \mathrm{mg}, 0.131 \mathrm{mmol})$ was loaded into a vial, and treated with a solution of 2b (50 mg, $0.131 \mathrm{mmol}$ ) in $1 \mathrm{~mL}$ of benzene at room temperature. The dark reaction mixture was transferred to a J-Young tube and monitored by ${ }^{31} \mathrm{P}\left\{{ }^{1} \mathrm{H}\right\}$ NMR spectroscopy. After heating at $60{ }^{\circ} \mathrm{C}$ for $120 \mathrm{~h}$, the solution turned dark red, and the ${ }^{31} \mathrm{P}\left\{{ }^{1} \mathrm{H}\right\} \mathrm{NMR}$ spectrum showed two doublets at $290.7 \mathrm{ppm}$ and $36.0 \mathrm{ppm}$. The mixture was subsequently filtered through Celite and the filtrate was concentrated under vacuum. The crude dark red solid was recrystallized from toluene layered with pentane at $-35{ }^{\circ} \mathrm{C}(83 \mathrm{mg}, 0.102 \mathrm{mmol}$, $78 \%$ yield). Crystals suitable for X-ray crystallography were collected by slow diffusion of pentane vapors into a concentrated toluene solution.

Anal. Calcd. for $\mathrm{C}_{47} \mathrm{H}_{59} \mathrm{Cl}_{2} \mathrm{NOP}_{2} \mathrm{Ru}$ [M+THF]: C, 63.58; H, 6.70; N, 1.58. Found: C, 63.24; $\mathrm{H}$, 6.85; N, 1.46. HRMS: $\mathrm{m} / \mathrm{z}$ was calcd for $\mathrm{C}_{48} \mathrm{H}_{51} \mathrm{~N}_{2} \mathrm{O}_{2} \mathrm{P}_{2} \mathrm{Ru}[\mathrm{M}]^{+}$: 815.1917. Found: 815.2510. ${ }^{31} \mathrm{P}\left\{{ }^{1} \mathrm{H}\right\} \mathrm{NMR}\left(\mathrm{CDCl}_{3}\right): \delta 290.7(\mathrm{~d}, J=36.4, \mathrm{C}=\mathrm{P}), 36.0\left(\mathrm{~d}, J=36.4, \mathrm{PPh}_{3}\right) .{ }^{1} \mathrm{H}$ NMR $\left(\mathrm{CDCl}_{3}\right): \delta$ $7.56(\mathrm{t}, J=8.0 \mathrm{~Hz}, 1 \mathrm{H}, \mathrm{Ar}), 7.46-7.39(\mathrm{~m}, 8 \mathrm{H}, \mathrm{Ar}), 7.38-7.32(\mathrm{~m}, 3 \mathrm{H}, \mathrm{Ar}), 7.26-7.22(\mathrm{~m}, 6 \mathrm{H}$, Ar), 6.92 (d, $J=7.5 \mathrm{~Hz}, 1 \mathrm{H}, \mathrm{Ar}$ ), 6.54 (d, $J=4.1 \mathrm{~Hz}, 1 \mathrm{H}, \mathrm{Ar}$ ), 2.85 (s, 3H, Me), 1.33 (s, 9H, $t$ $\mathrm{Bu}), 1.23$ (s, 18H, $t$-Bu). ${ }^{13} \mathrm{C}\left\{{ }^{1} \mathrm{H}\right\} \mathrm{NMR}\left(125 \mathrm{MHz}, \mathrm{CDCl}_{3}\right.$ ): $\delta 163.3$ (Ar), 159.5 (Ar), 155.2 (Ar), 153.4 (Ar), 136.0 (Ar), 134.7 (d, J=10.0 Hz, Ar), 133.2 (Ar), 132.9 (Ar), 129.5 (Ar), 127.8 (d, $J=10.0 \mathrm{~Hz}, \mathrm{Ar}$ ), 125.6 (d, $J=26.0 \mathrm{~Hz}, \mathrm{Ar}), 123.8$ (d, $J=10.0 \mathrm{~Hz}, \mathrm{Ar}$ ), 119.2 (d, $J=25.0 \mathrm{~Hz}$, $\mathrm{C}=\mathrm{P}), 119.0(\mathrm{Ar}), 39.8\left(\mathrm{CMe}_{3}\right), 35.2\left(\mathrm{CMe}_{3}\right), 33.9(t-\mathrm{Bu}), 31.0(t-\mathrm{Bu}), 24.7(\mathrm{Me})$. 
${ }^{31} \mathrm{P}\left\{{ }^{1} \mathrm{H}\right\} \mathrm{NMR}\left(\mathrm{CDCl}_{3}\right)$

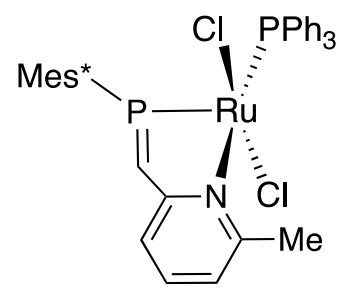

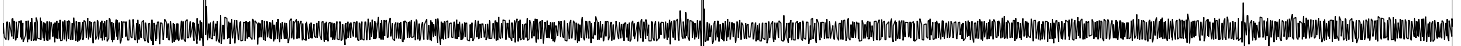

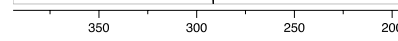

$200 \div \quad 150$

${ }^{31} \mathrm{P}\left\{{ }^{1} \mathrm{H}\right\} \mathrm{NMR}\left(\mathrm{CDCl}_{3}\right):$ Phosphaalkene
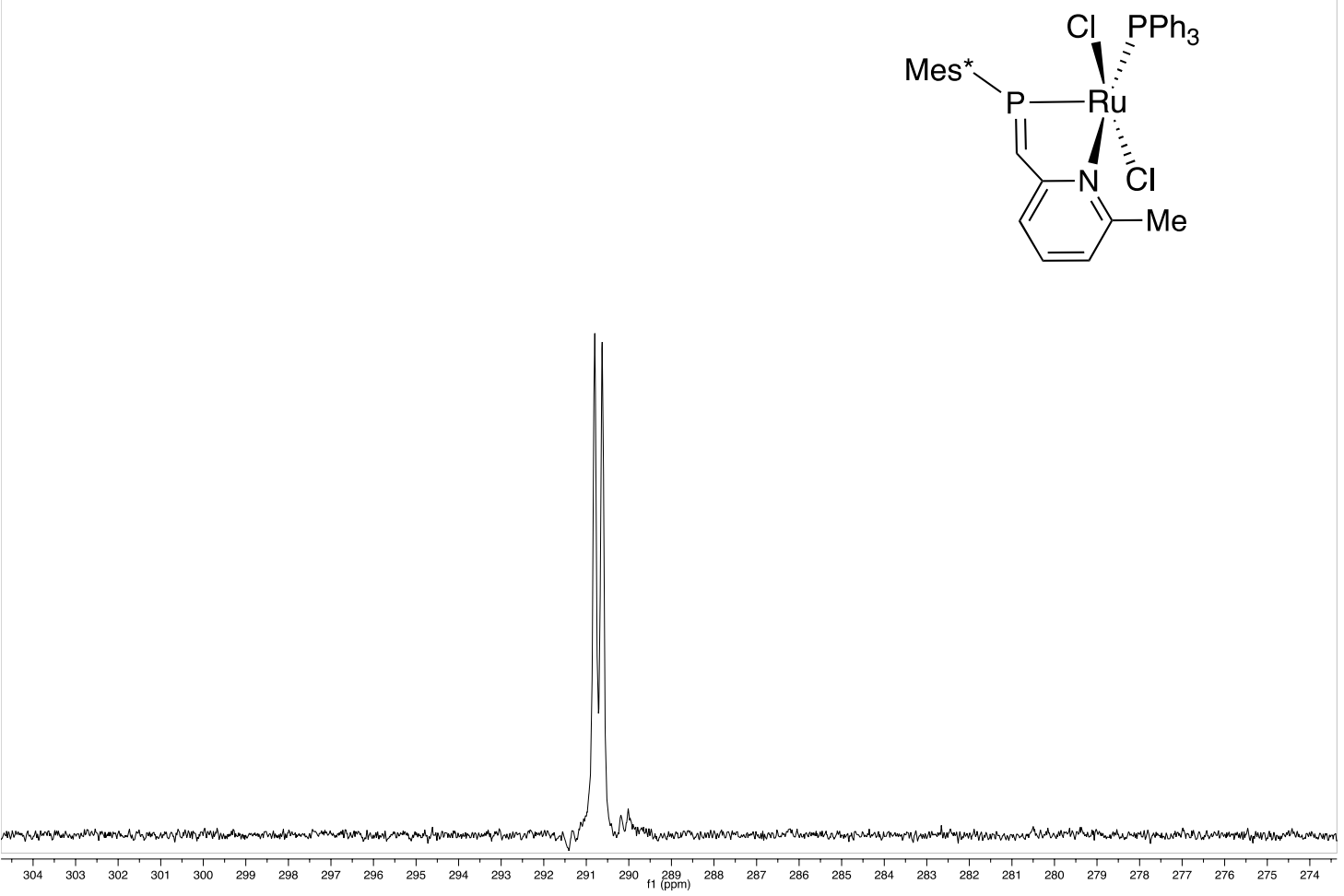
${ }^{31} \mathrm{P}\left\{{ }^{1} \mathrm{H}\right\}$ NMR $\left(\mathrm{CDCl}_{3}\right): \mathrm{PPh}_{3}$
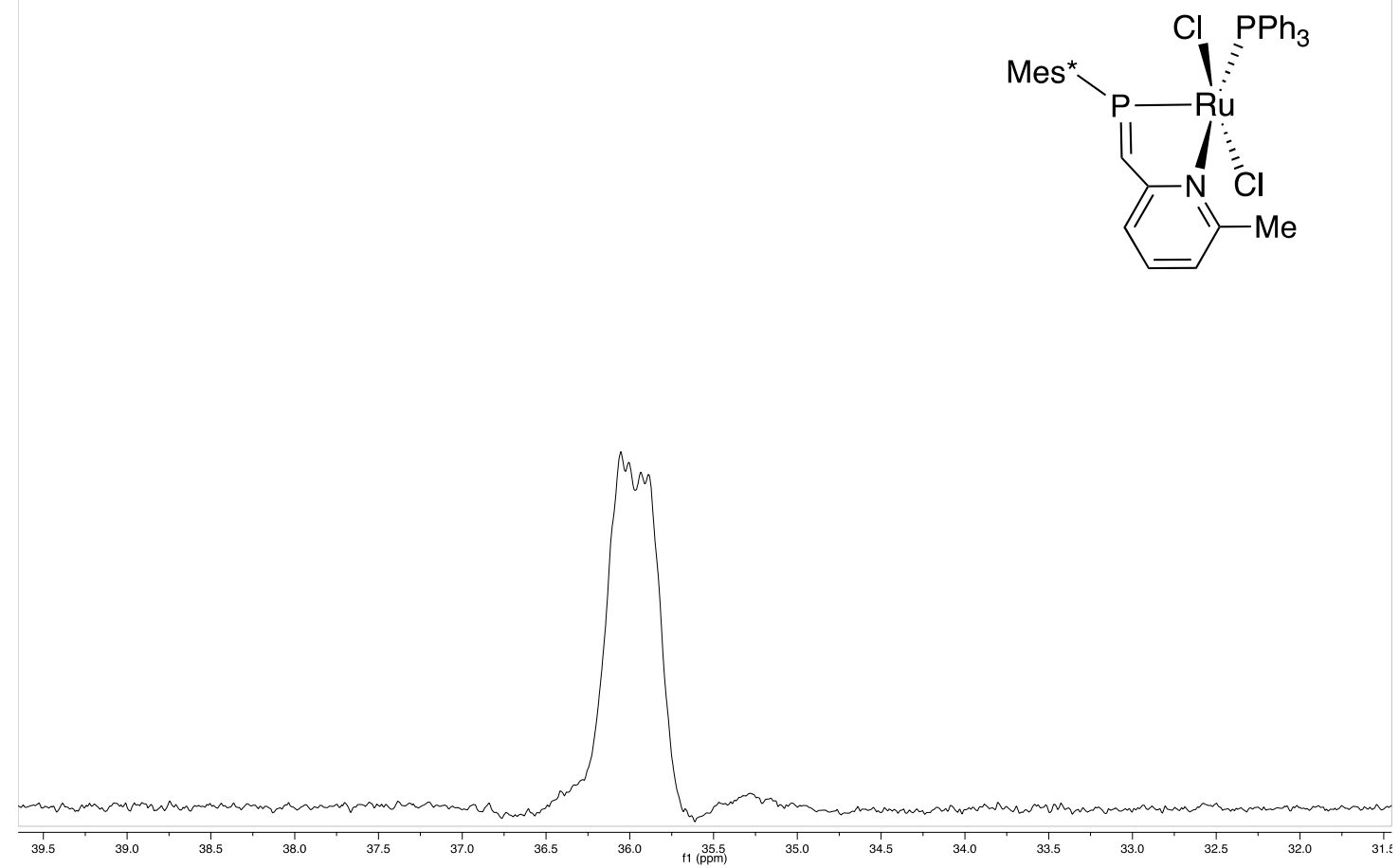

${ }^{1} \mathrm{H} \mathrm{NMR}\left(\mathrm{CDCl}_{3}\right)$

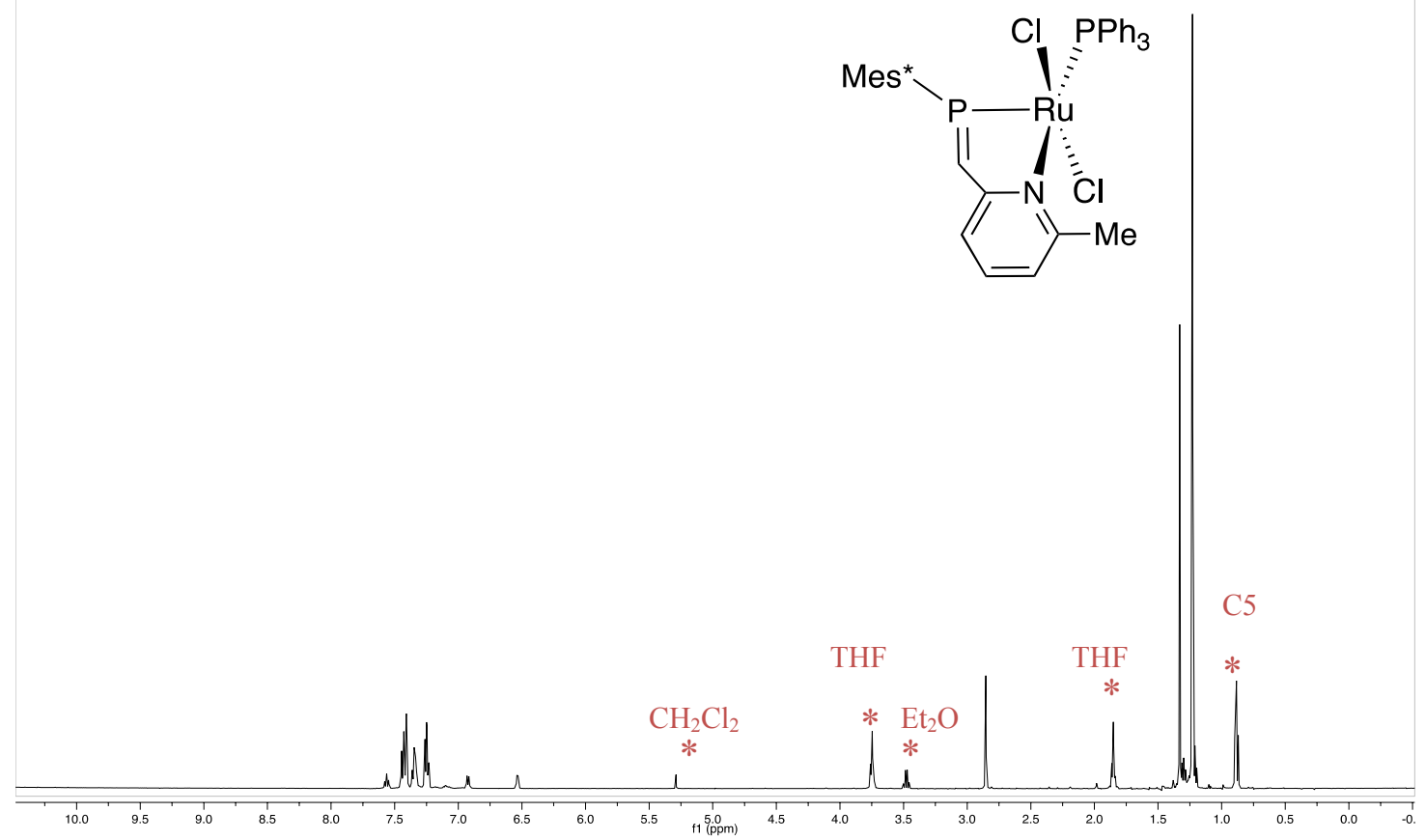


${ }^{1} \mathrm{H}$ NMR $\left(\mathrm{CDCl}_{3}\right)$ : Aryl and Phosphaalkene Region
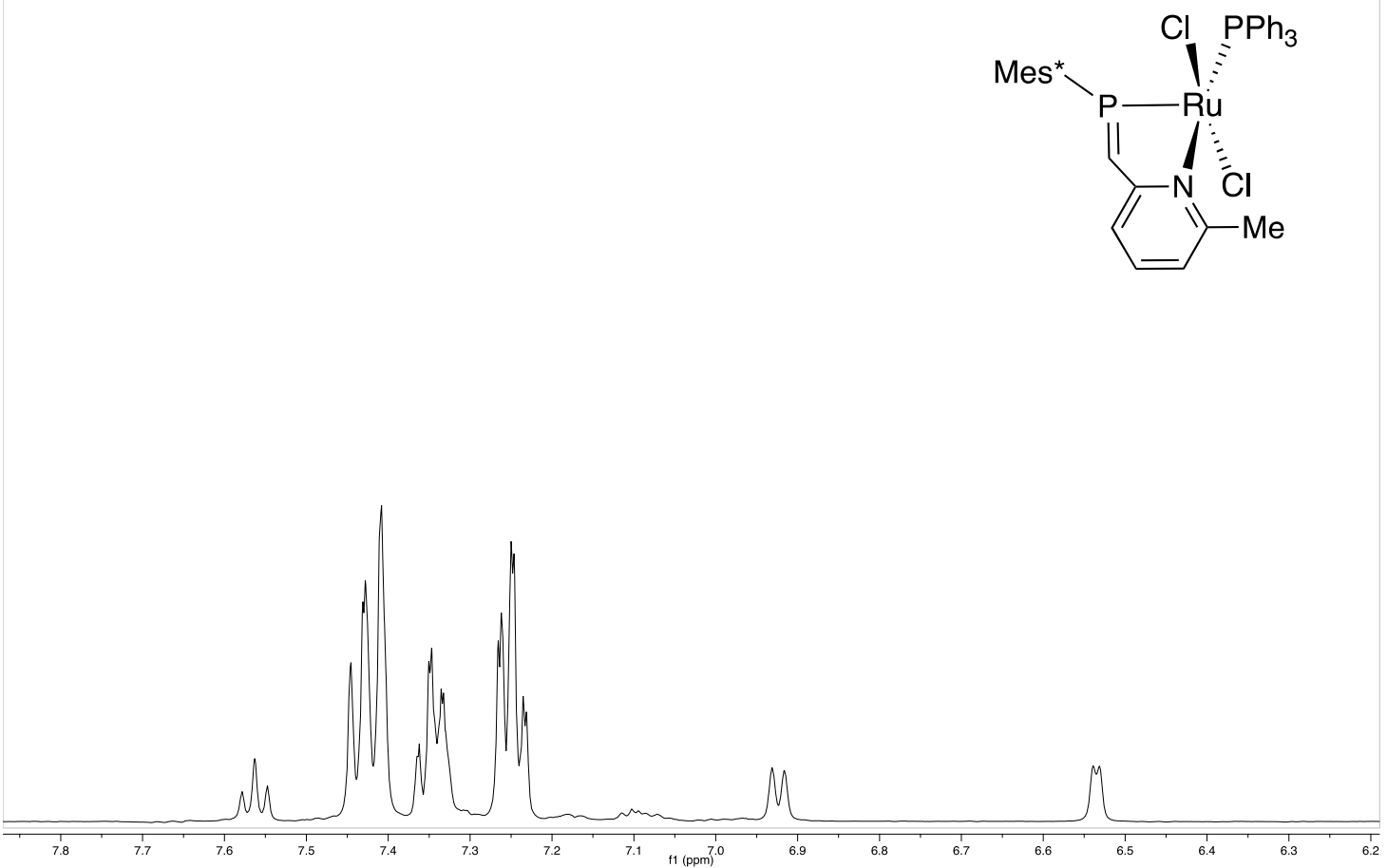

${ }^{13} \mathrm{C}\left\{{ }^{1} \mathrm{H}\right\}$ NMR $\left(\mathrm{CDCl}_{3}\right)$

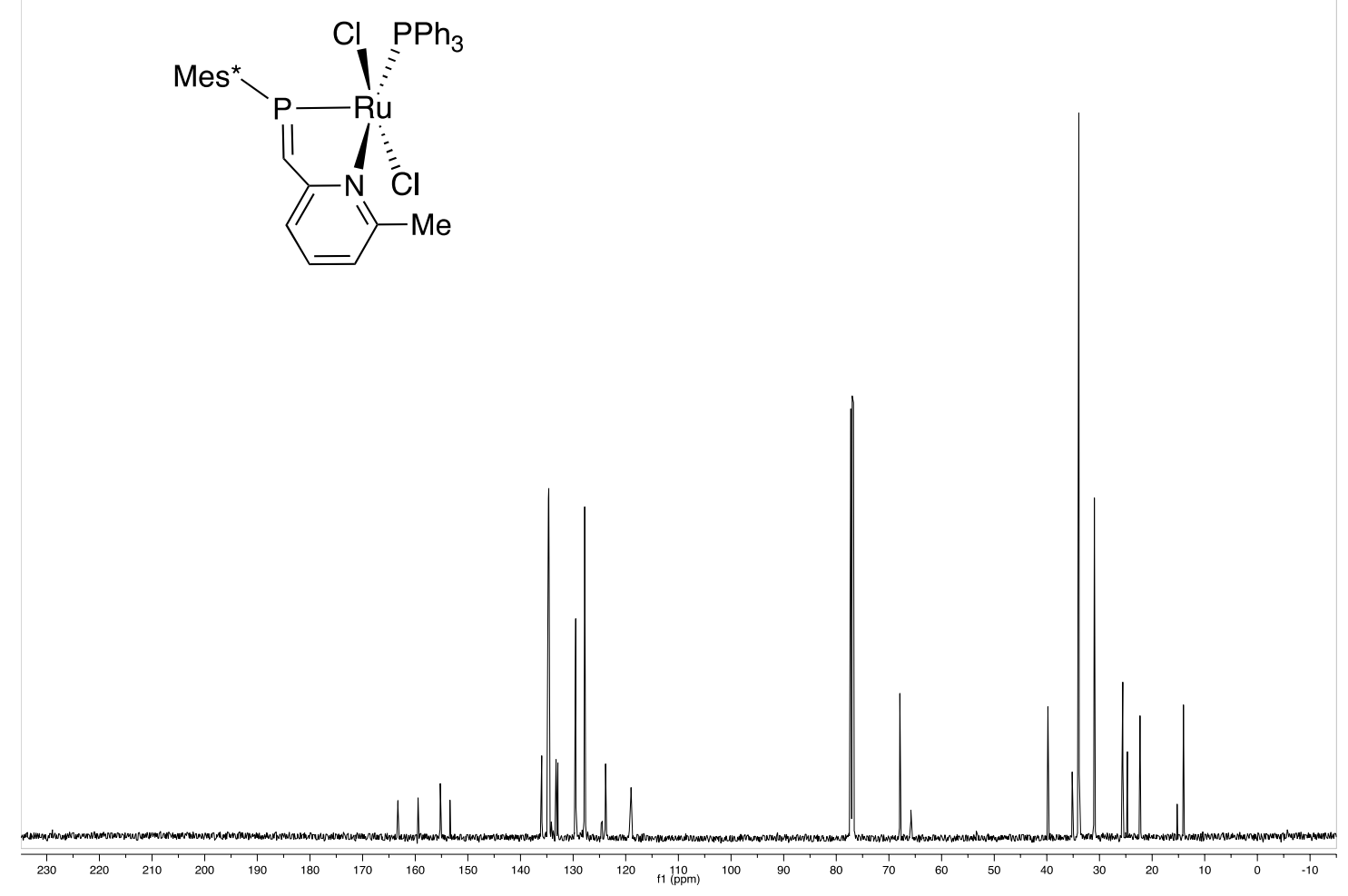


${ }^{13} \mathrm{C}\left\{{ }^{1} \mathrm{H}\right\}$ NMR $\left(\mathrm{CDCl}_{3}\right)$ : Aryl Region

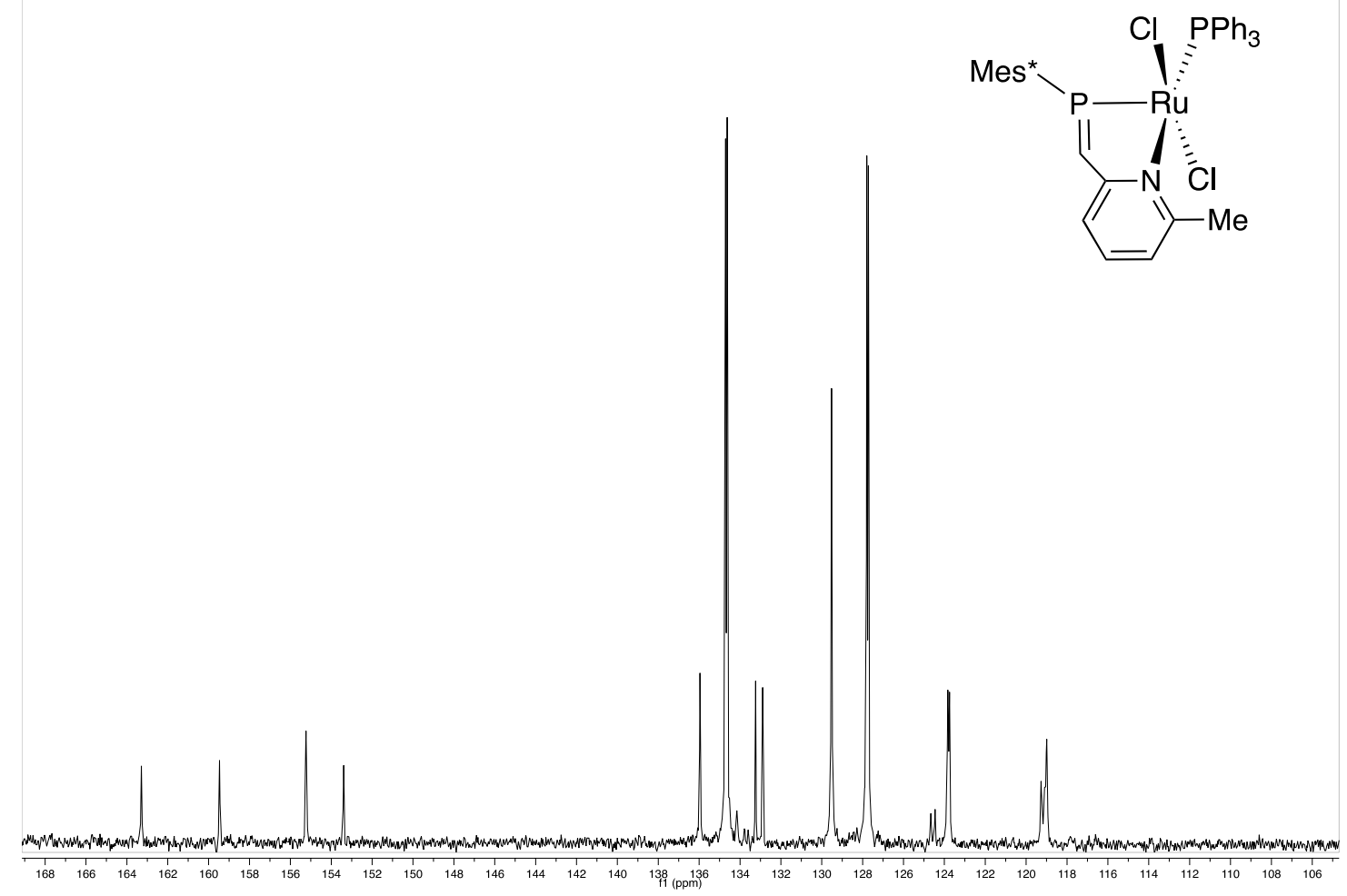

${ }^{13} \mathrm{C}\left\{{ }^{1} \mathrm{H}\right\}$ NMR $\left(\mathrm{CDCl}_{3}\right)$ : Alkyl Region

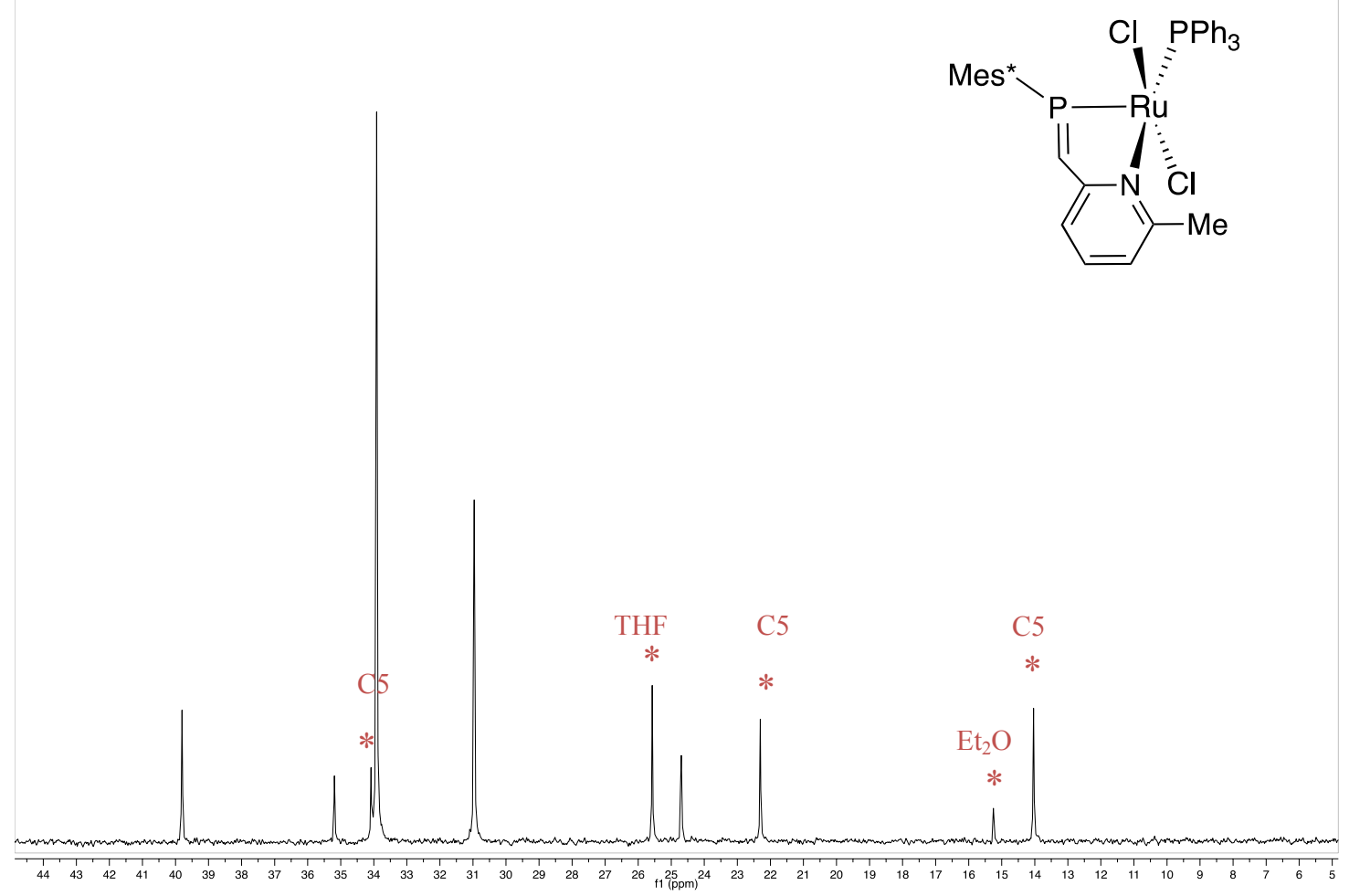




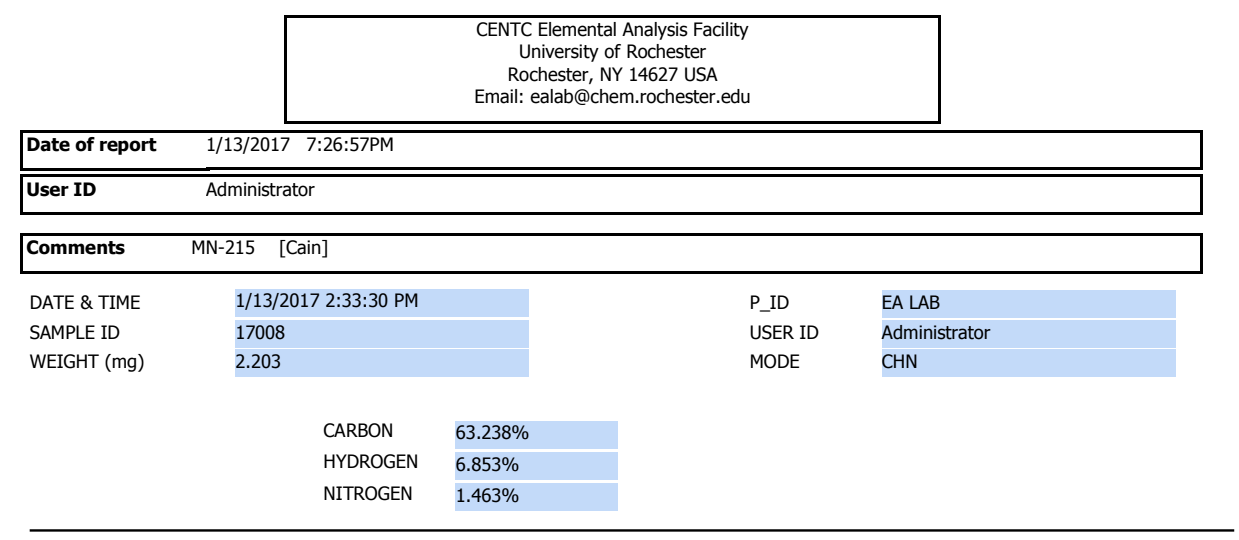

\section{Acknowledgment}

Analytical data were obtained from the CENTC Elemental Analysis Facility at the University of Rochester, funded by NSF CHE-0650456.

\section{Instrumentation}

Microanalysis samples were weighed with a PerkinElmer Model AD-6 Autobalance and their compositions were determined with a PerkinElmer 2400 Series II Analyzer.<smiles>Cc1cccc(C=[PH](c2c(C(C)(C)C)cc(C(C)(C)C)cc2C(C)(C)C)C(Cl)(Cl)c2ccccc2)n1</smiles>

Chemical Formula: $\mathrm{C}_{47} \mathrm{H}_{59} \mathrm{Cl}_{2} \mathrm{NOP}_{2} \mathrm{Ru}$

Exact Mass: 887.2492

Molecular Weight: 887.9125

Elemental Analysis: C, 63.58; $\mathrm{H}, 6.70 ; \mathrm{Cl}, 7.99$;

$\mathrm{N}, 1.58 ; \mathrm{O}, 1.80 ; \mathrm{P}, 6.98 ; \mathrm{Ru}, 11.38$

Crystallized as THF solvate (see $X$-ray section) 


\section{Synthesis of 3d $(\mathrm{R}=\mathrm{OMe})$}

$\mathrm{RuCl}_{2}\left(\mathrm{PPh}_{3}\right)_{3}$ (142 mg, $0.150 \mathrm{mmol}, 1.0$ equiv.) was loaded into a vial, and treated with a solution of $2 \mathbf{d}$ (60 mg, $0.150 \mathrm{mmol}, 1.0$ equiv.) in toluene at room temperature. The dark reaction mixture was transferred to a J-Young tube and monitored by ${ }^{31} \mathrm{P}\left\{{ }^{1} \mathrm{H}\right\}$ NMR spectroscopy. After heating at $100{ }^{\circ} \mathrm{C}$ for $120 \mathrm{~h}$, the solution turned dark red, and the ${ }^{31} \mathrm{P}\left\{{ }^{1} \mathrm{H}\right\}$ NMR spectrum showed two doublets at $296.6 \mathrm{ppm}$ and $34.0 \mathrm{ppm}$. The mixture was subsequently filtered through Celite and the filtrate was concentrated under vacuum. The dark red solid was recrystallized from toluene layered with pentane at $-35^{\circ} \mathrm{C}(65 \mathrm{mg}, 0.078 \mathrm{mmol}, 52 \%$ yield $)$.

${ }^{31} \mathrm{P}\left\{{ }^{1} \mathrm{H}\right\}$ NMR (202 MHz, $\left.\mathrm{CDCl}_{3}\right): \delta 296.6(\mathrm{~d}, J=36.4 \mathrm{~Hz}, \mathrm{C}=\mathrm{P}), 34.0\left(\mathrm{~d}, J=36.4 \mathrm{~Hz}, \mathrm{PPh}_{3}\right) .{ }^{1} \mathrm{H}$ NMR (500 MHz, $\left.\mathrm{CDCl}_{3}\right): \delta 7.65(\mathrm{t}, J=8.0 \mathrm{~Hz}, 1 \mathrm{H}, \mathrm{Ar}), 7.48-7.40(\mathrm{~m}, 8 \mathrm{H}, \mathrm{Ar}), 7.37-7.31(\mathrm{~m}$, $3 \mathrm{H}, \mathrm{Ar}), 7.27-7.21$ (m, 5H, Ar), 7.18 (d, $J=7.5 \mathrm{~Hz}, 1 \mathrm{H}, \mathrm{Ar}), 7.11$ (d, $J=7.5 \mathrm{~Hz}, 1 \mathrm{H}, \mathrm{Ar}), 6.51$ $(\mathrm{d}, J=8.0 \mathrm{~Hz}, 1 \mathrm{H}, \mathrm{Ar}), 6.39$ (s, 1H, Ar), 4.07 (s, 3H, OMe), 1.37 (s, 9H, $t$-Bu), 1.87 (s, 18H, $t$ $\mathrm{Bu}) .{ }^{13} \mathrm{C}\left\{{ }^{1} \mathrm{H}\right\} \mathrm{NMR}\left(125 \mathrm{MHz}, \mathrm{CDCl}_{3}\right)$ : $\delta 167.1$ (Ar), 162.4 (Ar), 155.4 (Ar), 153.2 (Ar), 138.6 (Ar), 134.8 (d, $J=10.0 \mathrm{~Hz}, \mathrm{Ar}$ ), 134.0 (d, $J=43.8 \mathrm{~Hz}, \mathrm{Ar}$ ), 132.9 (Ar), 132.5 (Ar), 129.4 (Ar), 129.0 (Ar), 128.2 (Ar), 127.7 (d, $J=10.0 \mathrm{~Hz}, \mathrm{Ar}), 123.8$ (d, $J=10.0 \mathrm{~Hz}, \mathrm{Ar}), 114.4$ (d, $J=23.8$ $\mathrm{Hz}, \mathrm{P}=\mathrm{C}), 99.9(\mathrm{Ar}), 56.6(\mathrm{OMe}), 39.6\left(\mathrm{CMe}_{3}\right), 35.2\left(\mathrm{CMe}_{3}\right), 34.1(t-\mathrm{Bu}), 31.1(t-\mathrm{Bu})$. 

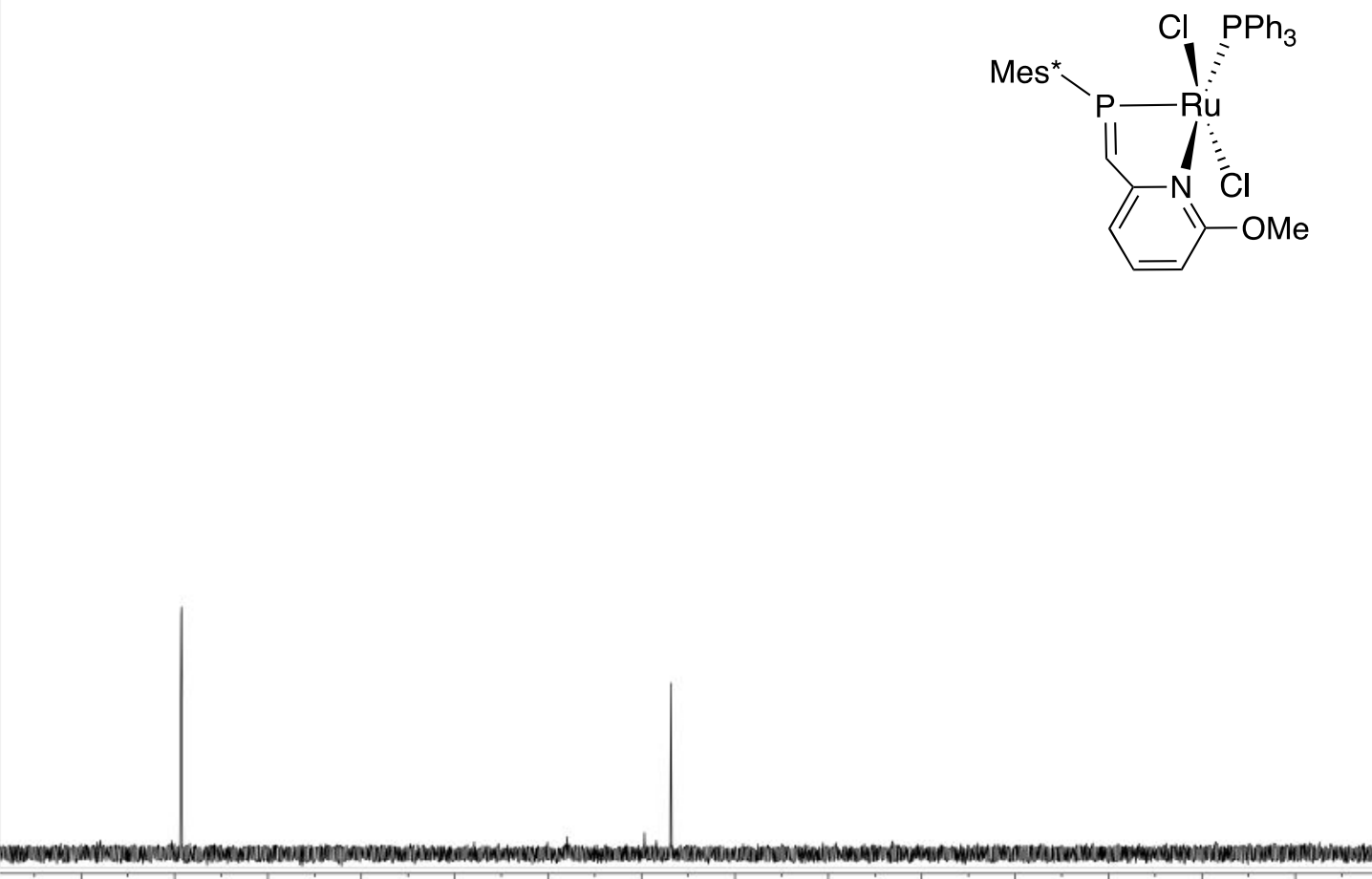

${ }^{31} \mathrm{P}\left\{{ }^{1} \mathrm{H}\right\} \mathrm{NMR}\left(\mathrm{CDCl}_{3}\right)$ : Phosphaalkene

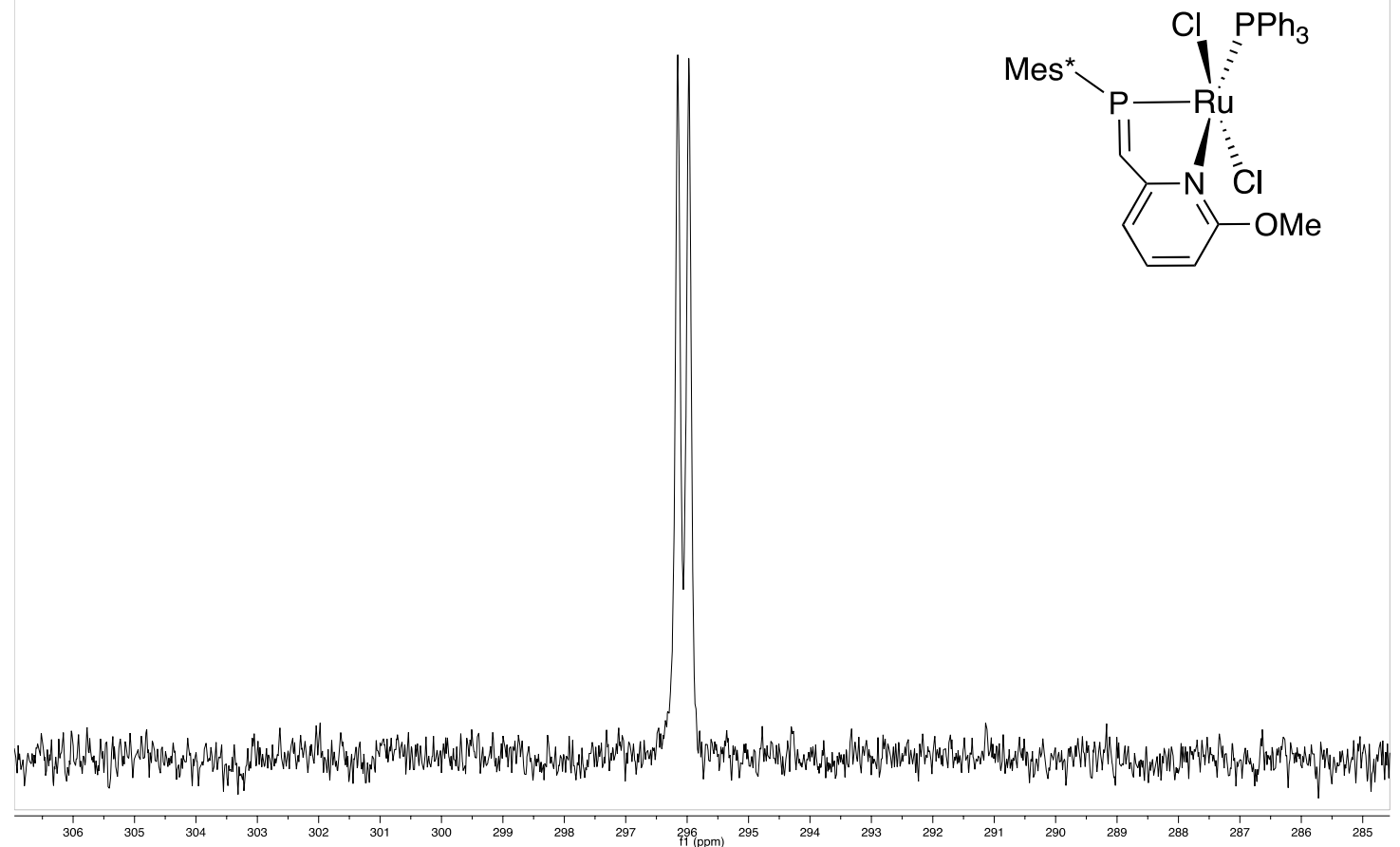


${ }^{31} \mathrm{P}\left\{{ }^{1} \mathrm{H}\right\} \mathrm{NMR}\left(\mathrm{CDCl}_{3}\right): \mathrm{PPh}_{3}$
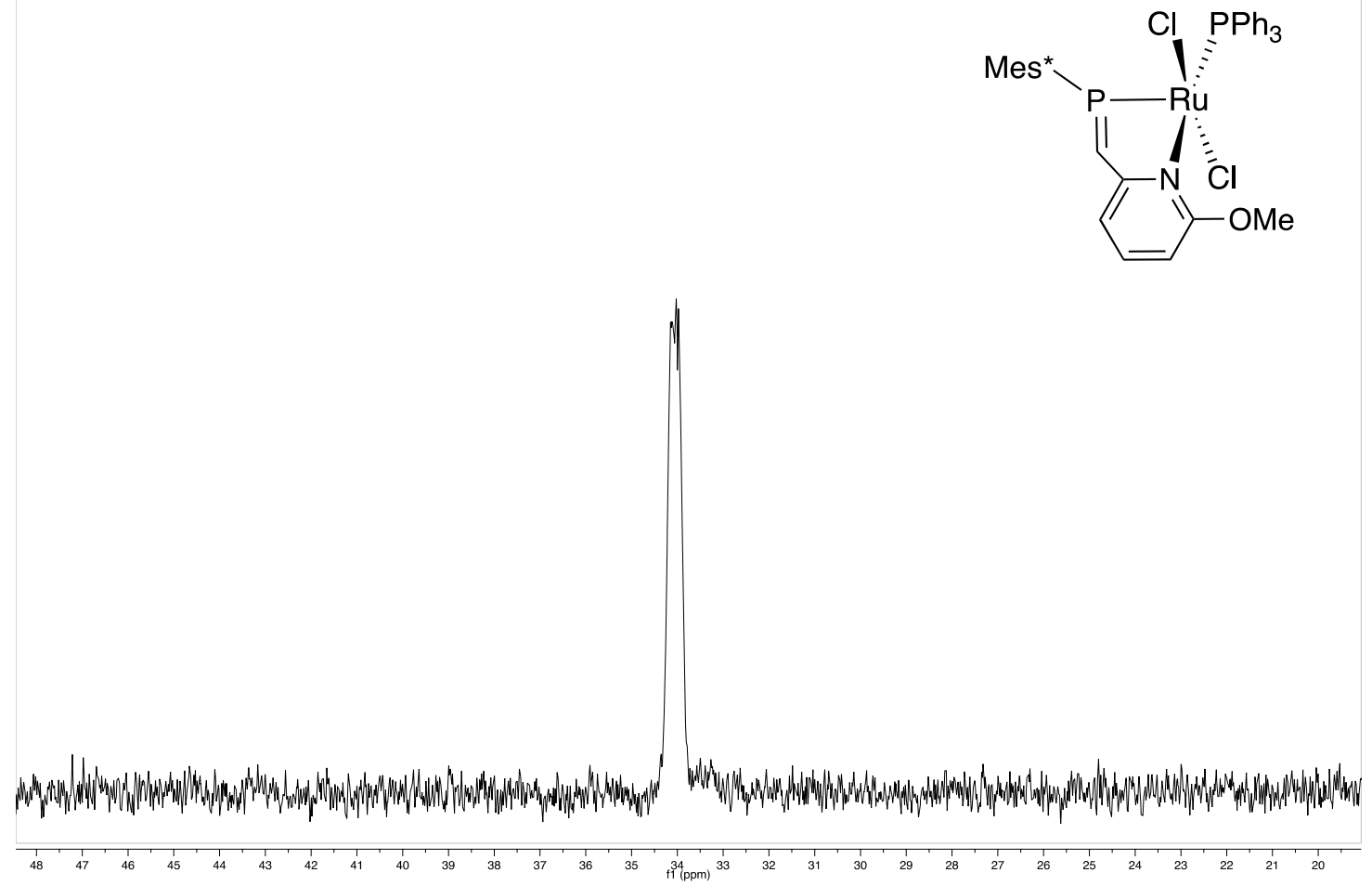

${ }^{1} \mathrm{H} \mathrm{NMR}\left(\mathrm{CDCl}_{3}\right)$

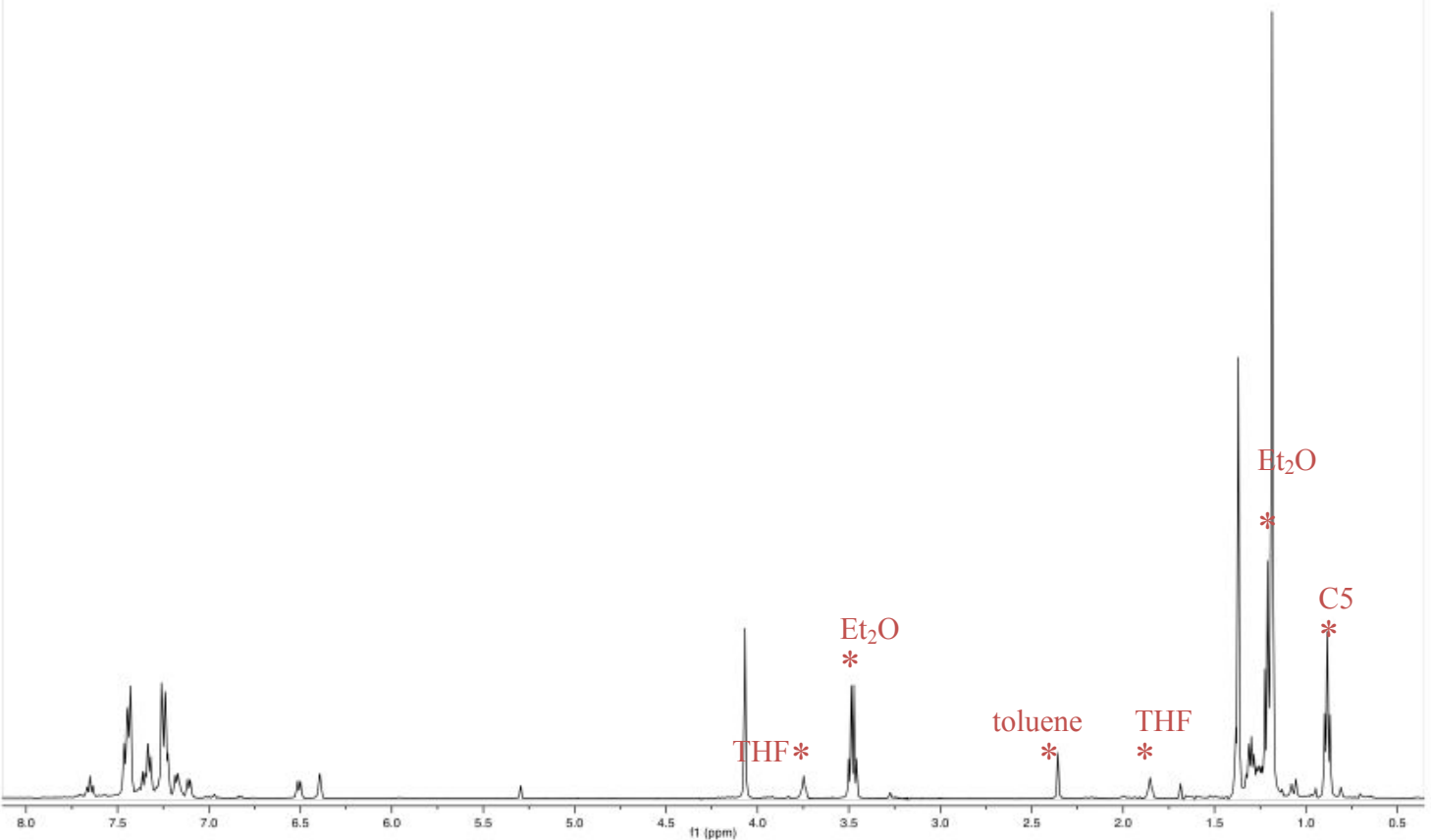


${ }^{1} \mathrm{H}$ NMR $\left(\mathrm{CDCl}_{3}\right)$ : Aryl and Phosphaalkene Region
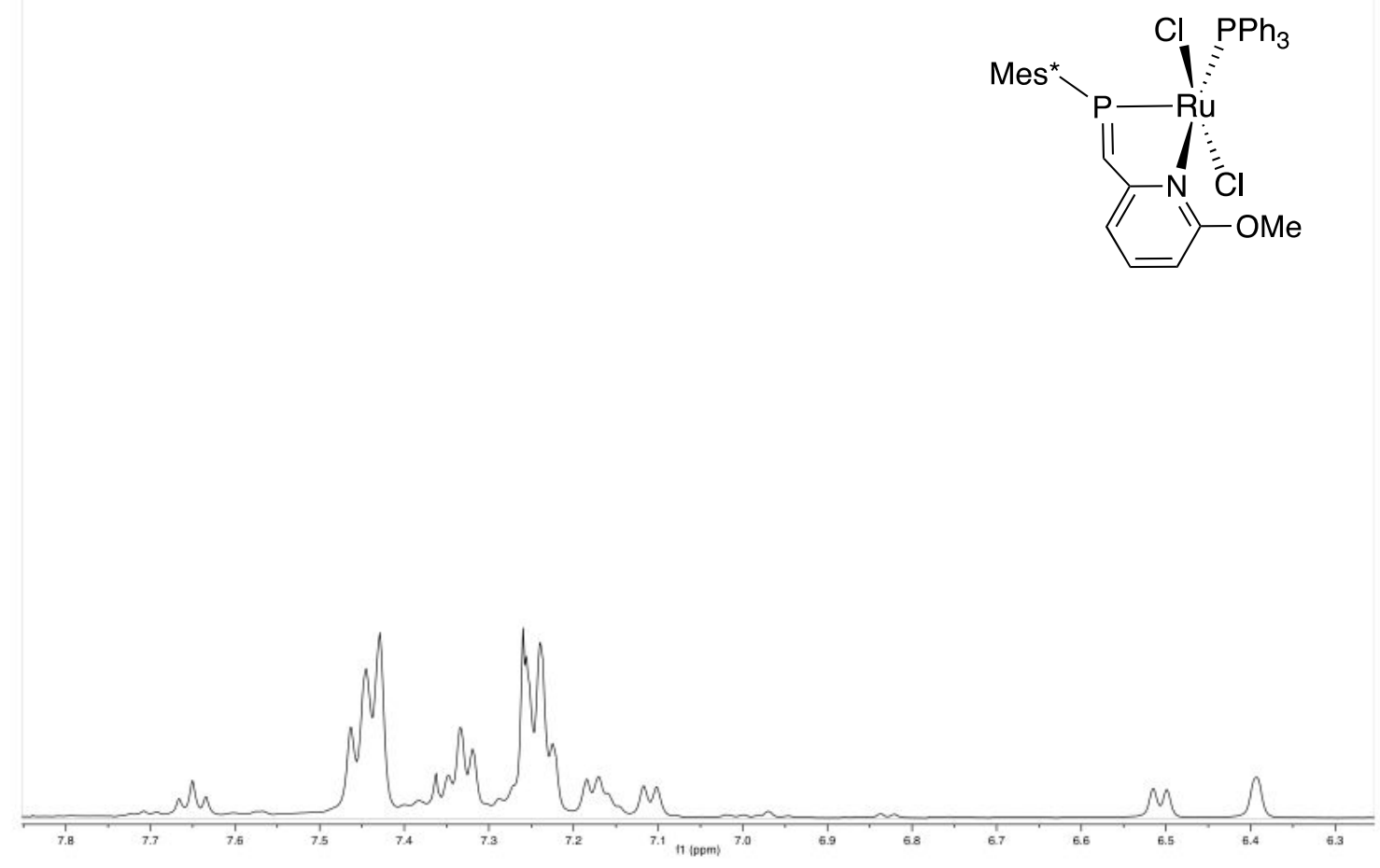

${ }^{13} \mathrm{C}\left\{{ }^{1} \mathrm{H}\right\}$ NMR $\left(\mathrm{CDCl}_{3}\right)$

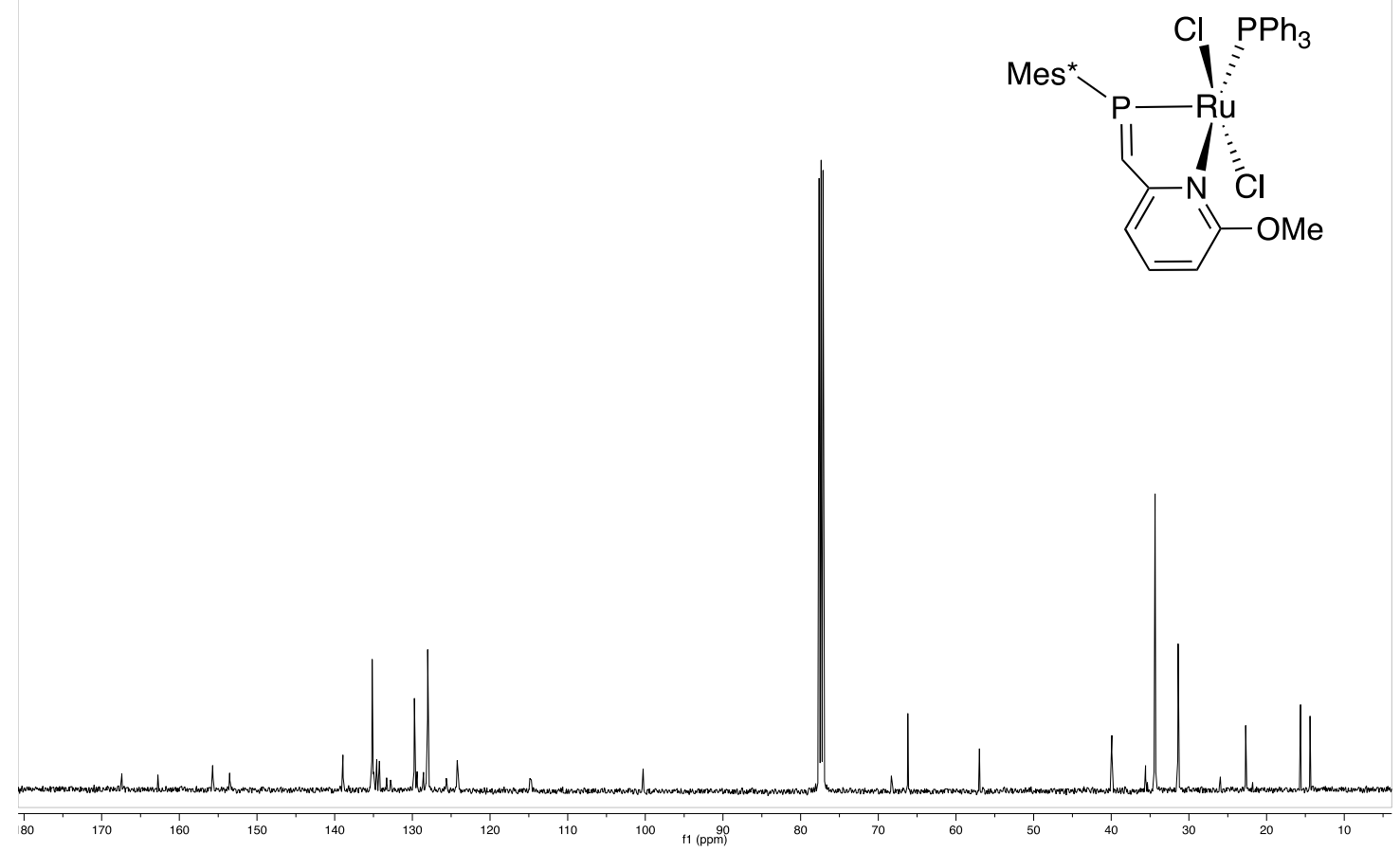


${ }^{13} \mathrm{C}\left\{{ }^{1} \mathrm{H}\right\}$ NMR $\left(\mathrm{CDCl}_{3}\right)$ : Aryl Region

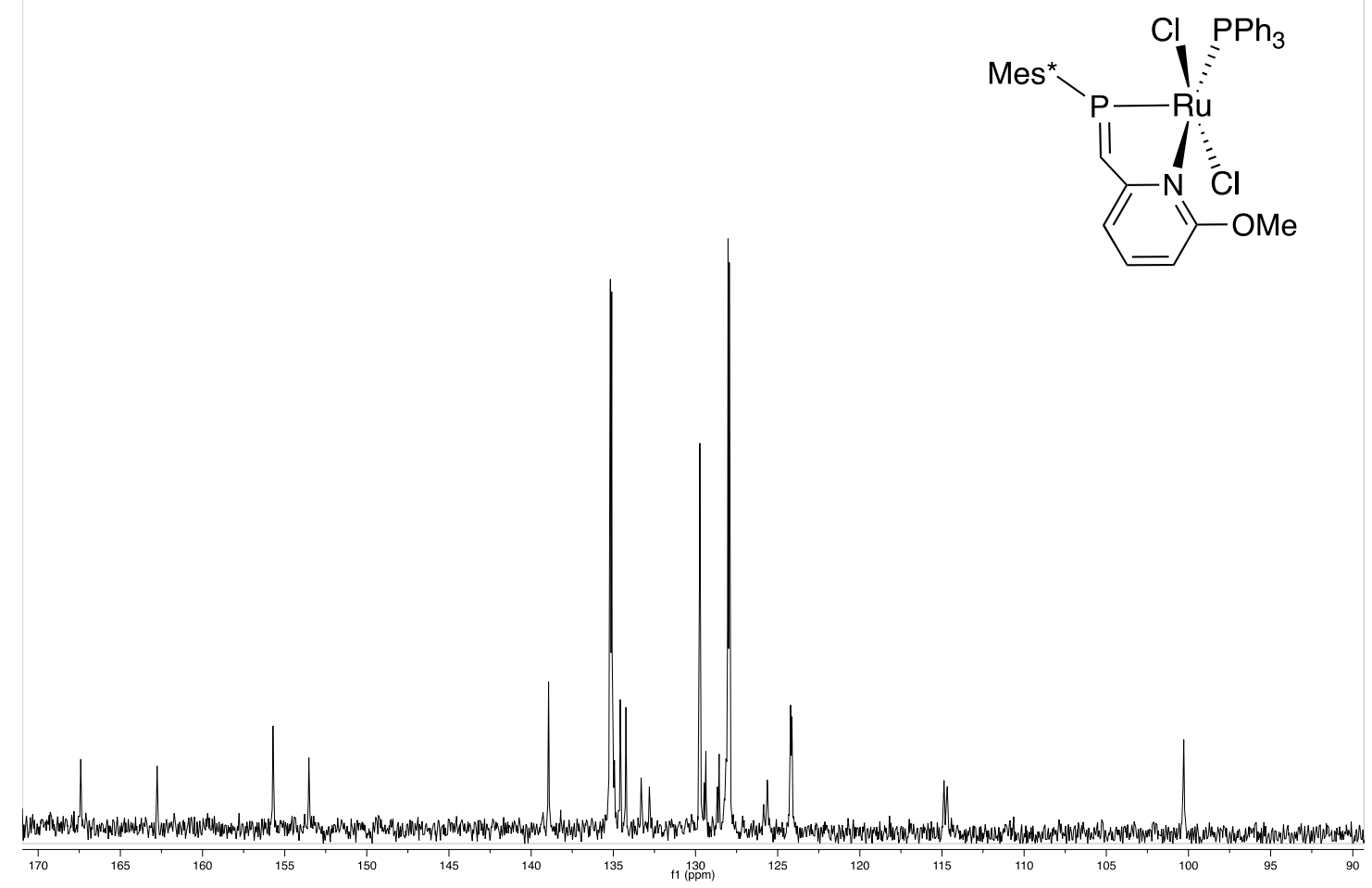

${ }^{13} \mathrm{C}\left\{{ }^{1} \mathrm{H}\right\}$ NMR $\left(\mathrm{CDCl}_{3}\right)$ : Alkyl Region

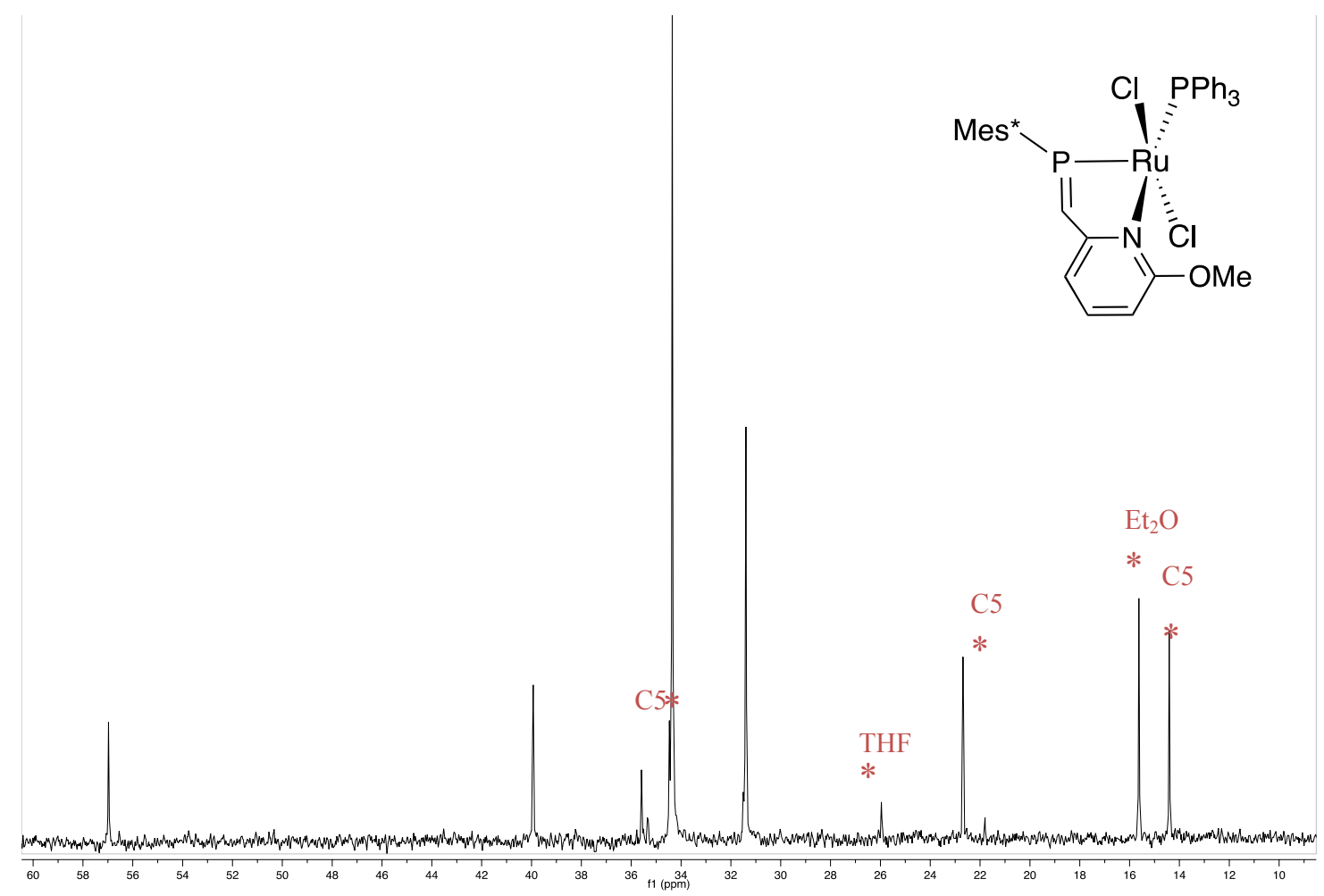




\section{Synthesis of $4 \mathbf{b}(\mathrm{R}=\mathrm{Me})$}

A solution of $\mathbf{3 b}(15 \mathrm{mg}, 0.019 \mathrm{mmol})$ in $1 \mathrm{~mL}$ of THF was heated at $80{ }^{\circ} \mathrm{C}$ for $48 \mathrm{~h}$ at which time the phosphaalkene resonance no longer was observed by ${ }^{31} \mathrm{P}$ NMR spectroscopy. The cyclized complex ( ${ }^{31} \mathrm{P}$ NMR: $\delta$ 91.1, 46.7) was filtered through a pad of Celite and the filtrate was concentrated under vacuum. The crude solid was recrystallized from a 1:3 solution of THF:pentane at $-35{ }^{\circ} \mathrm{C}$ to give dark green crystals $(12 \mathrm{mg}, 0.015 \mathrm{mmol}, 80 \%$ yield). Crystals suitable for X-ray crystallography were collected by slow diffusion of pentane vapors into a concentrated THF solution.

HRMS: $\mathrm{m} / \mathrm{z}$ was calcd for $\mathrm{C}_{45} \mathrm{H}_{54} \mathrm{ClN}_{2} \mathrm{P}_{2} \mathrm{Ru}\left[\mathrm{M}-\left(\mathrm{Cl}^{-}\right)+\mathrm{NCMe}\right]^{+}$: 821.2494. Found: 821.2516. ${ }^{31} \mathrm{P}\left\{{ }^{1} \mathrm{H}\right\}$ NMR $\left(202 \mathrm{MHz}, \mathrm{CDCl}_{3}\right): \delta 91.1\left(\mathrm{br}, \mathrm{CH}_{2} \mathrm{P}\right), 48.7\left(\mathrm{~d}, J=36.4 \mathrm{~Hz}, \mathrm{PPh}_{3}\right) .{ }^{1} \mathrm{H}$ NMR $(500$ $\mathrm{MHz}, \mathrm{CDCl}_{3}$ ): $\delta$ 7.61-7.56 (m, 2H, Ar), 7.40 (t, $\left.J=9.0 \mathrm{~Hz}, 6 \mathrm{H}, \mathrm{Ar}\right), 7.31$ (t, $J=8.5 \mathrm{~Hz}, 4 \mathrm{H}, \mathrm{Ar}$ ), 7.21-7.14 (m, 7H, Ar), $6.96(\mathrm{~d}, J=1.5 \mathrm{~Hz}, 1 \mathrm{H}, \mathrm{Ar}), 4.23(\mathrm{dd}, J=17.0,11.0 \mathrm{~Hz}, 1 \mathrm{H}$, benzylic $\mathrm{N}_{\mathrm{Ar}}$ ), 3.99 (dd, $J=17.0,10.0 \mathrm{~Hz}, 1 \mathrm{H}$, benzylic $\left.\mathrm{N}_{\mathrm{Ar}}\right), 2.80(\mathrm{~s}, 3 \mathrm{H}, \mathrm{Me}), 2.17-1.96\left(\mathrm{~m}, 2 \mathrm{H}, \mathrm{CH}_{2} \mathrm{P}\right)$, $1.38(\mathrm{~s}, 9 \mathrm{H}, t-\mathrm{Bu}), 1.23(\mathrm{~s}, 9 \mathrm{H}, t-\mathrm{Bu}), 1.10\left(\mathrm{~s}, 3 \mathrm{H}, \mathrm{CMe}_{2}\right), 0.79\left(\mathrm{~s}, 3 \mathrm{H}, \mathrm{CMe}_{2}\right) .{ }^{13} \mathrm{C}\left\{{ }^{1} \mathrm{H}\right\} \mathrm{NMR}$ (125 MHz, $\mathrm{CDCl}_{3}$ ): $\delta 162.1$ (Ar), $160.4(\mathrm{Ar}), 157.3$ (d, $J=21.3$, Ar), 156.7 (d, $J=7.5 \mathrm{~Hz}, \mathrm{Ar}$ ), 153.6 (Ar), 136.2 (Ar), 134.6 (d $J=10.0 \mathrm{~Hz}, \mathrm{Ar}), 133.7$ (d, $J=42.5 \mathrm{~Hz}, \mathrm{Ar}$ ), 129.3 (Ar), 129.0 (Ar), 128.3 (d, $J=12.5 \mathrm{~Hz}, \mathrm{Ar}), 127.9$ (d, $J=8.8 \mathrm{~Hz}, \mathrm{Ar}), 126.8$ (d, $J=10.0 \mathrm{~Hz}, \mathrm{Ar}), 123.8$ (Ar), 119.2 (d, $J=10.0 \mathrm{~Hz}, \mathrm{Ar}), 117.9$ (d, $J=10.0 \mathrm{~Hz}, \mathrm{Ar}), 50.9$ (d, $J=22.5 \mathrm{~Hz}$, benzylic), 47.6 (d, $J$ $\left.=43.8 \mathrm{~Hz}, \mathrm{PCH}_{2} \mathrm{CMe}_{2}\right), 43.2\left(\mathrm{CMe}_{2}\right), 37.9\left(\mathrm{CMe}_{3}\right), 35.4(t-\mathrm{Bu}), 35.0\left(\mathrm{CMe}_{3}\right), 34.1(t-\mathrm{Bu}), 31.3$ $(t-\mathrm{Bu}), 28.6\left(\mathrm{~d}, J=10.0 \mathrm{~Hz}, \mathrm{CMe}_{2}\right)$, 
${ }^{31} \mathrm{P}\left\{{ }^{1} \mathrm{H}\right\} \mathrm{NMR}\left(\mathrm{CDCl}_{3}\right)$
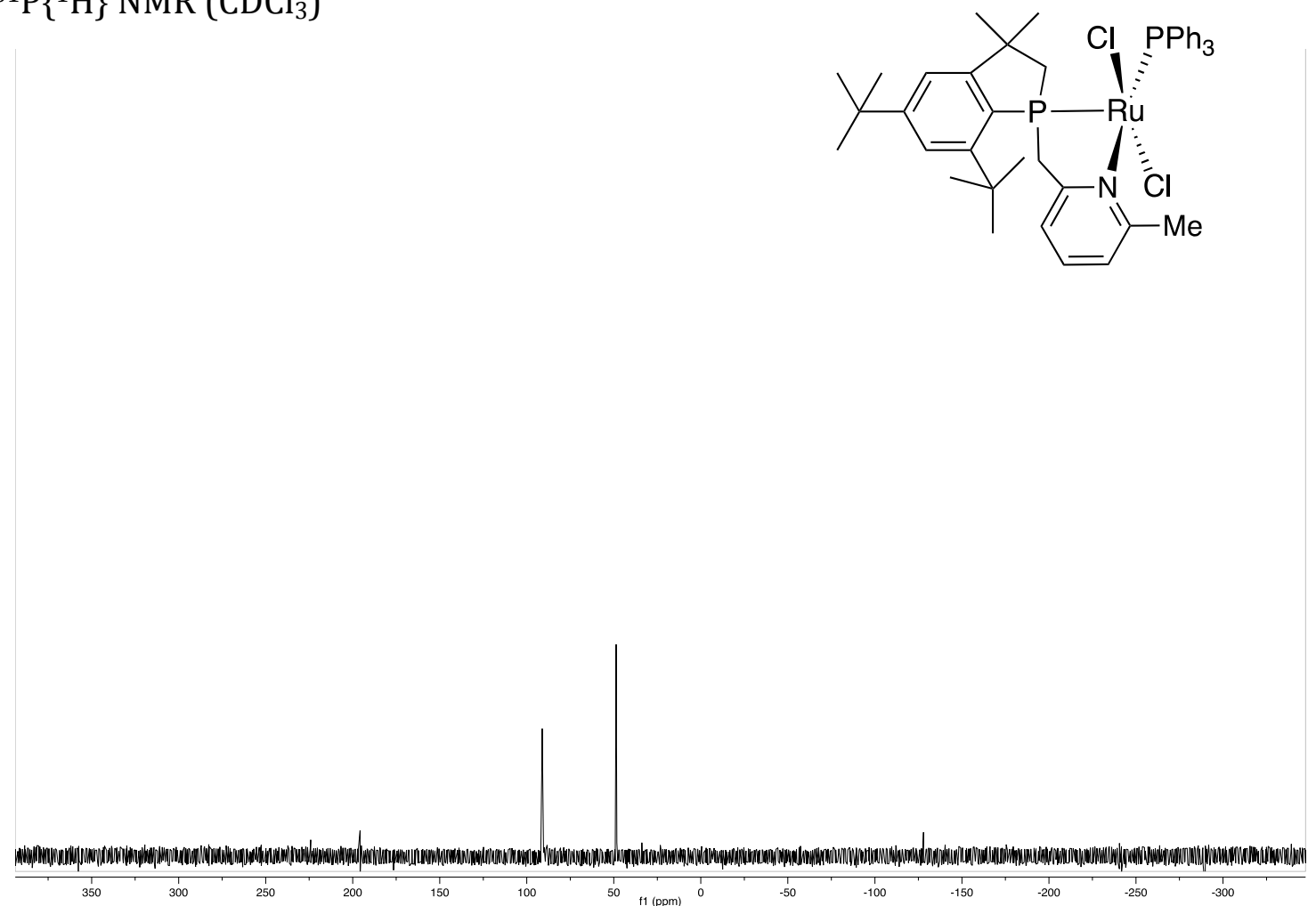

${ }^{31} \mathrm{P}\left\{{ }^{1} \mathrm{H}\right\} \mathrm{NMR}\left(\mathrm{CDCl}_{3}\right):$
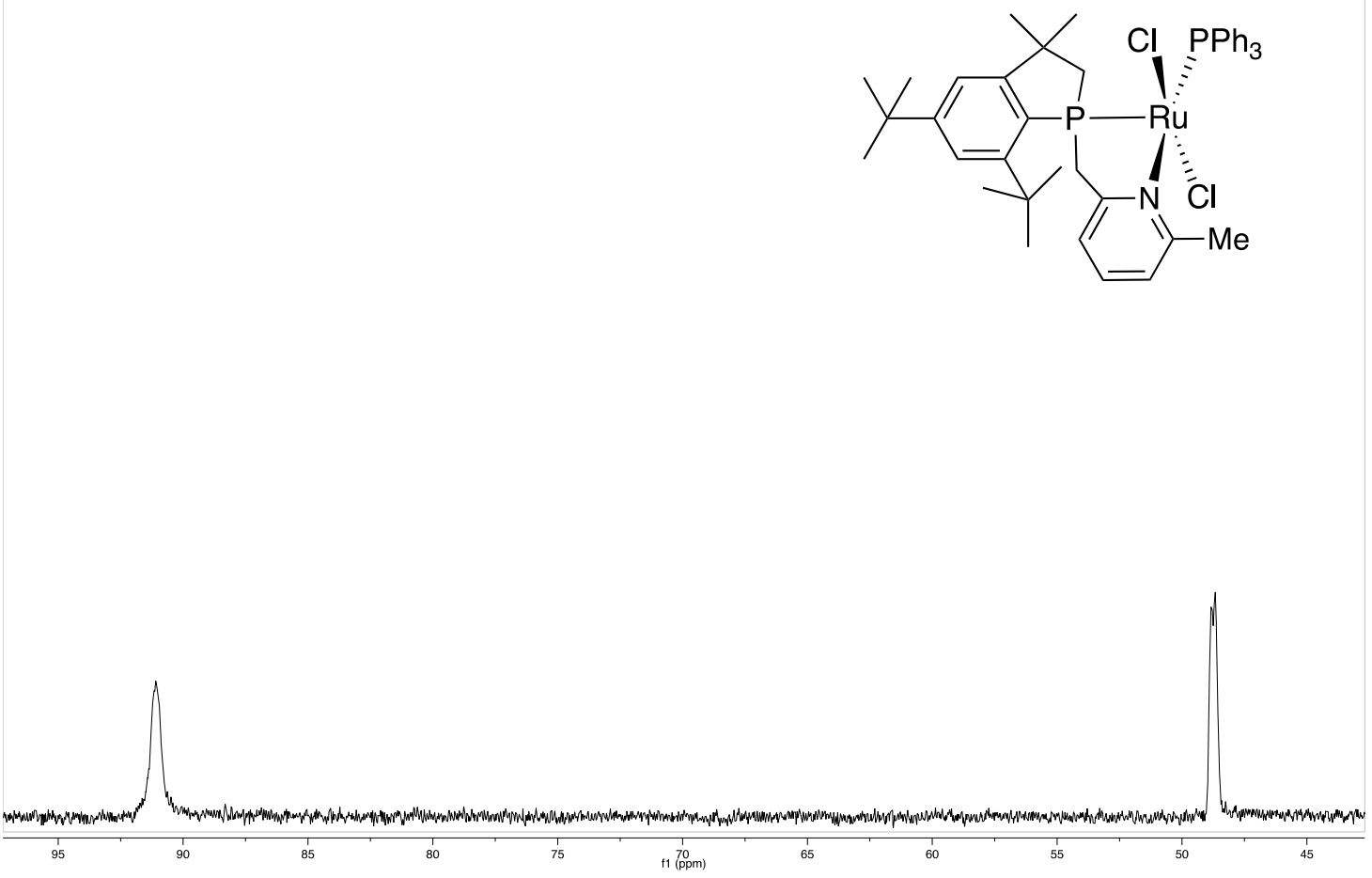


\section{${ }^{1} \mathrm{H}$ NMR $\left(\mathrm{CDCl}_{3}\right)$}

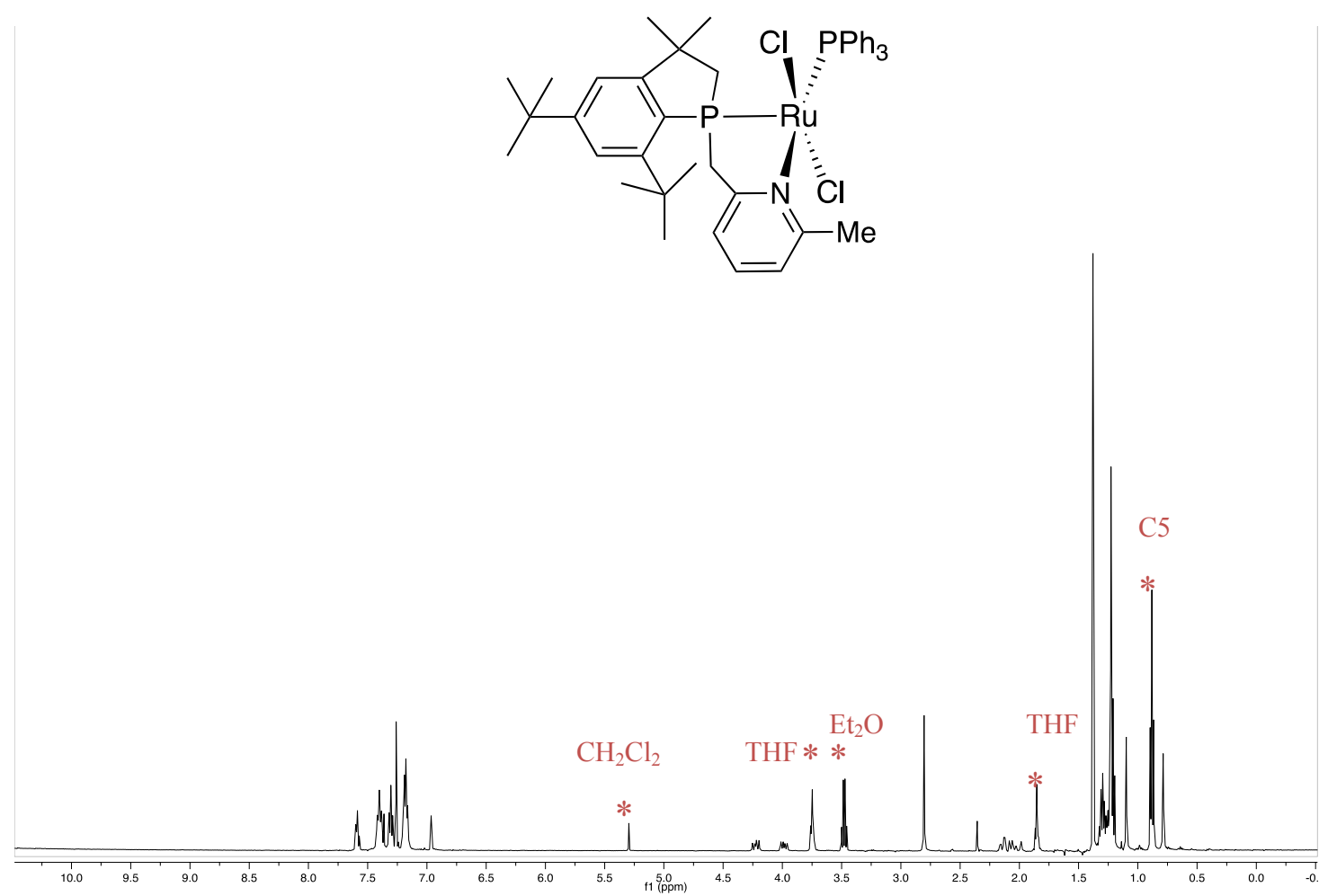

${ }^{1} \mathrm{H}$ NMR $\left(\mathrm{CDCl}_{3}\right)$ : Benzylic Protons
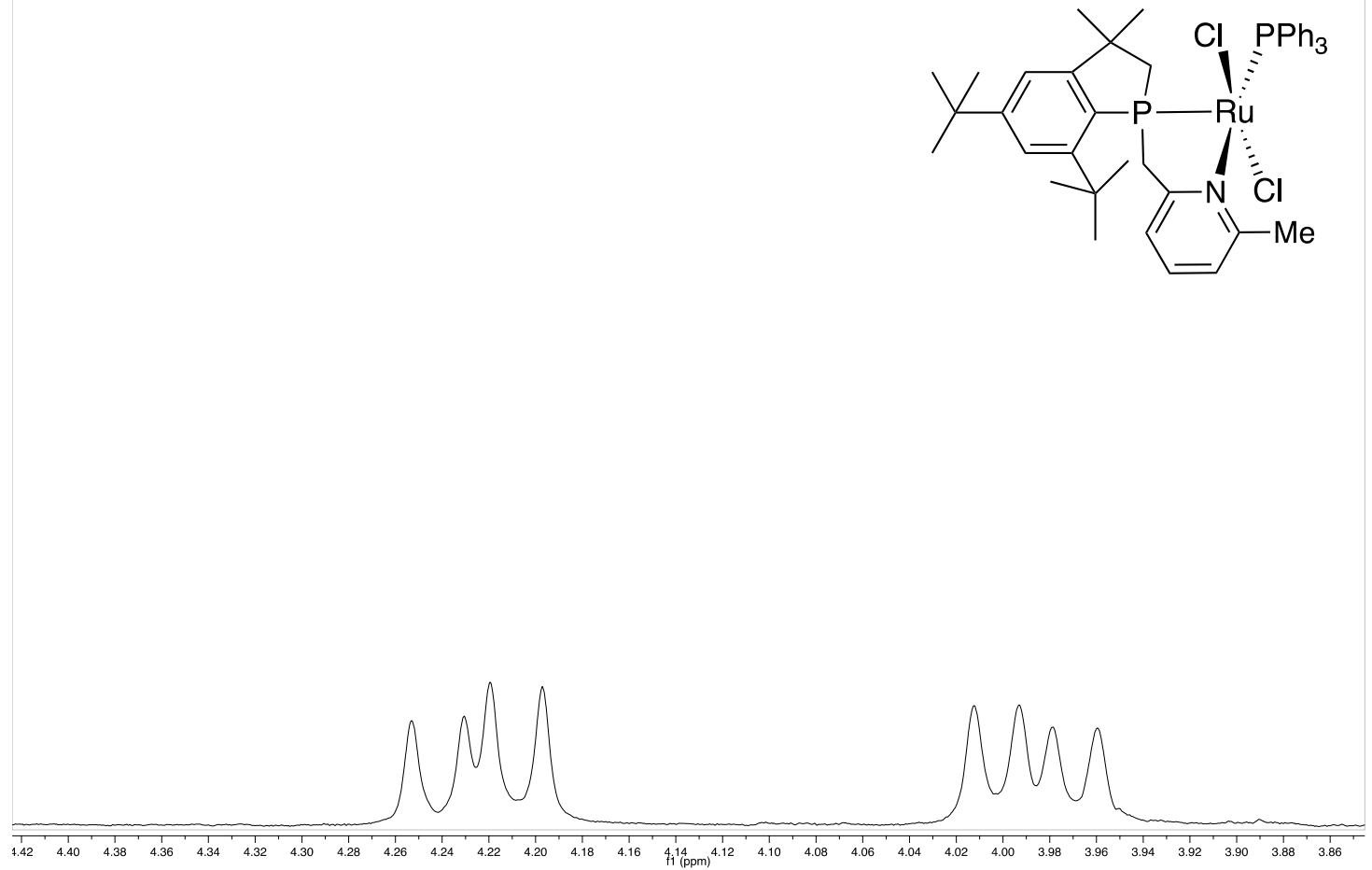
${ }^{1} \mathrm{H}$ NMR $\left(\mathrm{CDCl}_{3}\right)$ : Phospholane Protons
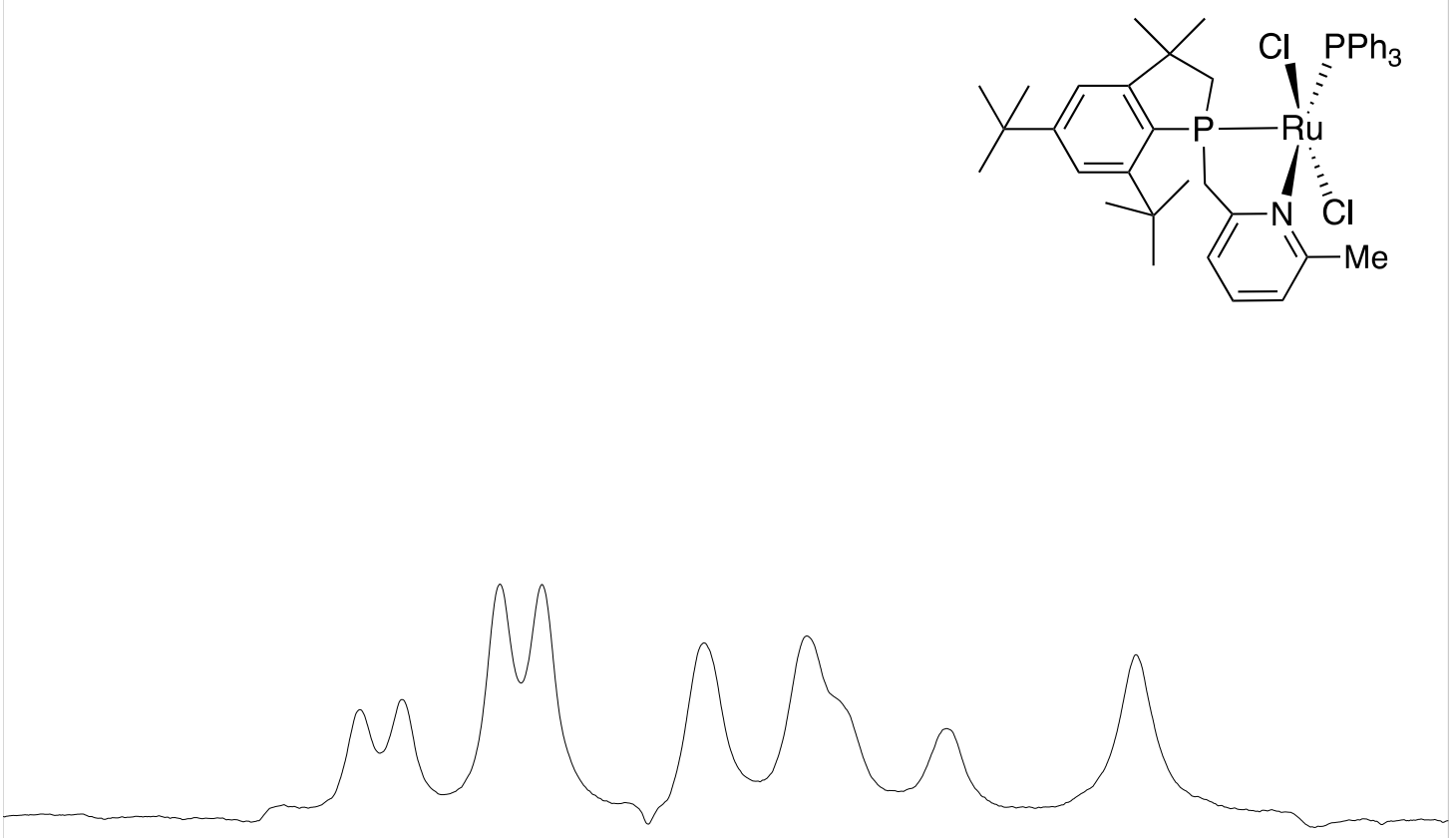

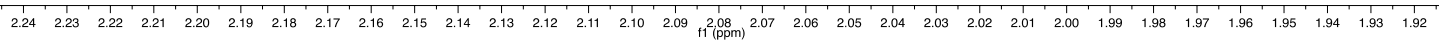

${ }^{13} \mathrm{C}\left\{{ }^{1} \mathrm{H}\right\}$ NMR $\left(\mathrm{CDCl}_{3}\right)$

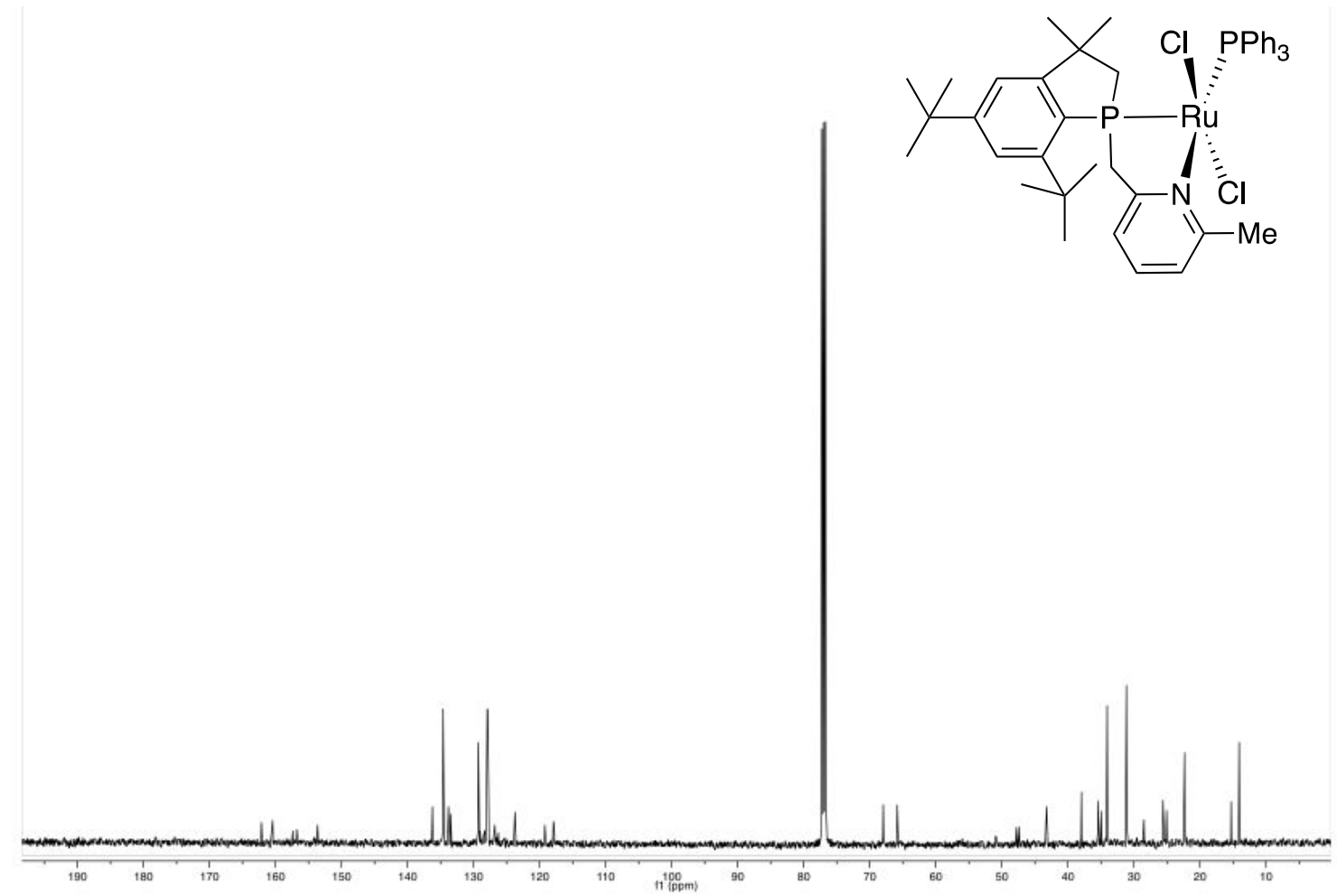


${ }^{13} \mathrm{C}\left\{{ }^{1} \mathrm{H}\right\}$ NMR $\left(\mathrm{CDCl}_{3}\right)$ : Aryl Region
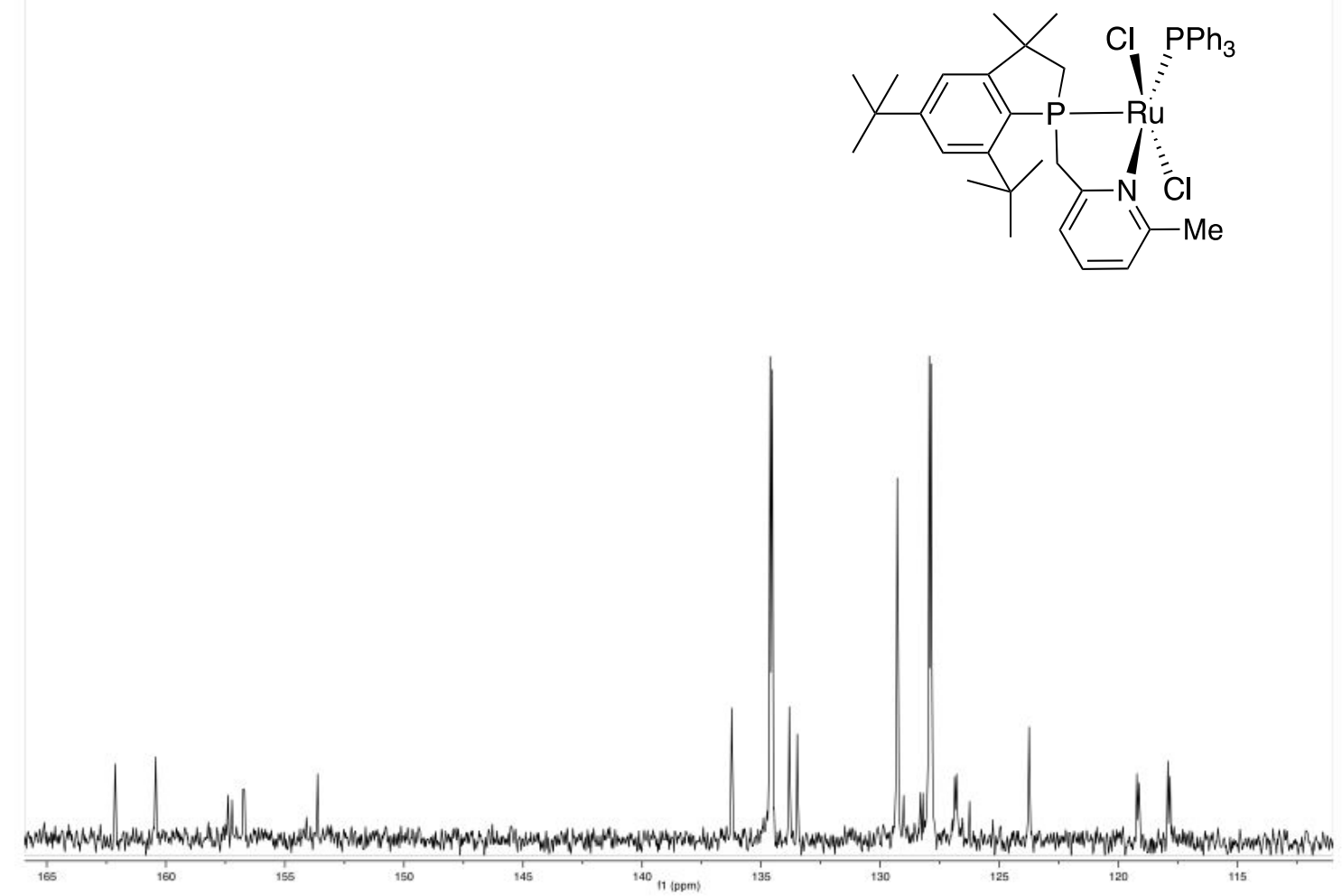

${ }^{13} \mathrm{C}\left\{{ }^{1} \mathrm{H}\right\}$ NMR $\left(\mathrm{CDCl}_{3}\right)$ : Alkyl Region

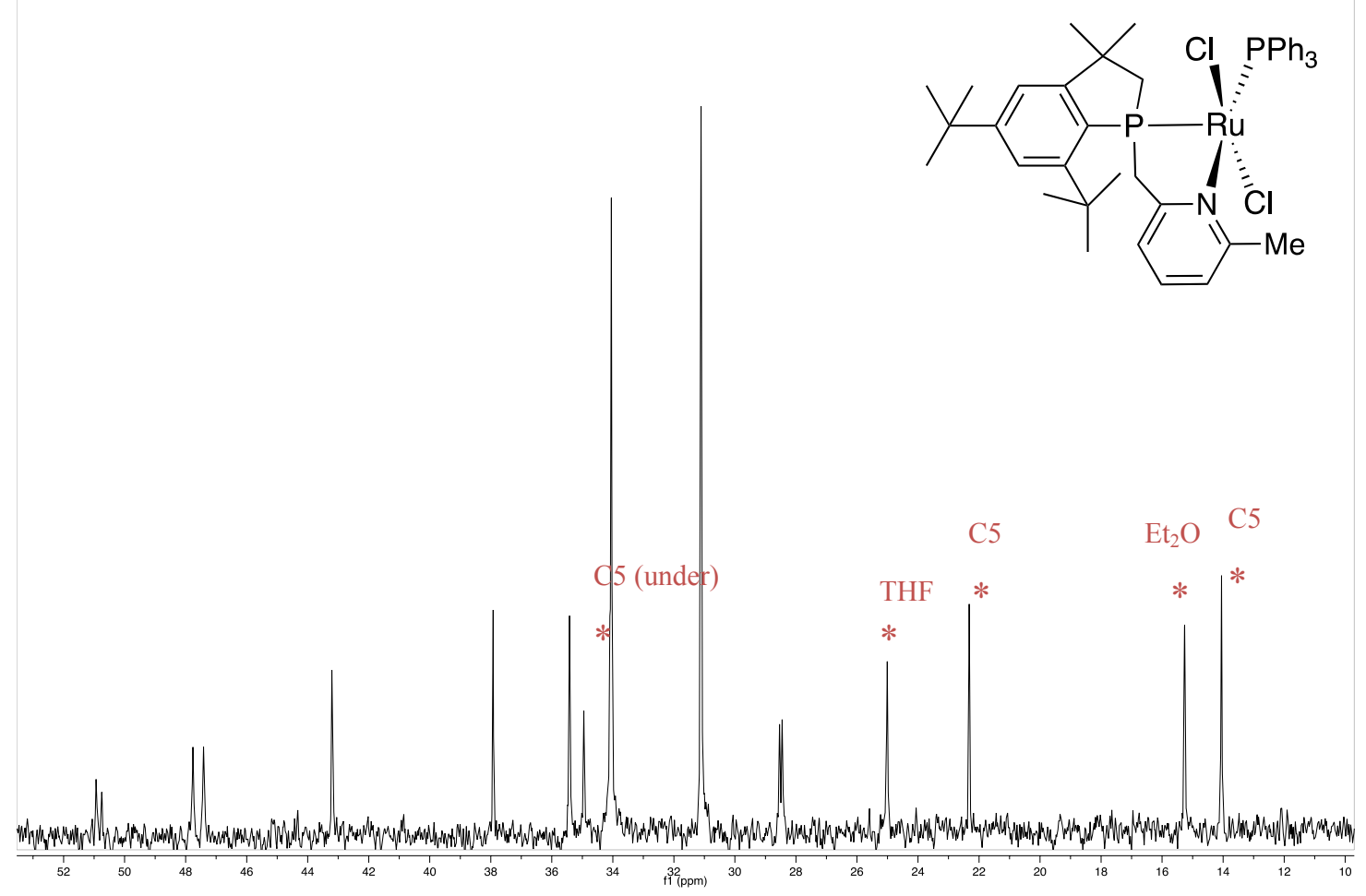




\section{Synthesis of $4 d(\mathrm{R}=\mathrm{OMe})$}

A solution of $\mathbf{3 d}(20 \mathrm{mg}, 0.024 \mathrm{mmol})$ in $1 \mathrm{~mL}$ of a 1:1 mixture of THF:toluene was heated at $100{ }^{\circ} \mathrm{C}$ for $336 \mathrm{~h}$ at which time the phosphaalkene resonance no longer was observed by ${ }^{31} \mathrm{P}$ NMR spectroscopy. The cyclized complex $\left({ }^{31} \mathrm{P}\right.$ NMR: $\left.\delta 91.8,49.7\right)$ was filtered through a pad of Celite and the filtrate was concentrated under vacuum. The crude solid was recrystallized from a 1:3 solution of THF:pentane at $-35{ }^{\circ} \mathrm{C}$ to afford dark green crystals $(7 \mathrm{mg}, 0.008 \mathrm{mmol}, 35 \%$ yield).

HRMS: $\mathrm{m} / \mathrm{z}$ was calcd for $\mathrm{C}_{43} \mathrm{H}_{51} \mathrm{ClNOP}_{2} \mathrm{Ru}^{+}[\mathrm{M}-(\mathrm{Cl}-)]^{+}:$796.2178. Found 796.2189. ${ }^{31} \mathrm{P}\left\{{ }^{1} \mathrm{H}\right\}$ NMR (202 MHz, $\left.\mathrm{C}_{6} \mathrm{D}_{6}\right): \delta 91.8\left(\mathrm{~d}, J=46.1 \mathrm{~Hz}, \mathrm{PCH}_{2}\right), 49.7\left(\mathrm{~d}, J=46.1 \mathrm{~Hz}, \mathrm{PPh}_{3}\right) .{ }^{1} \mathrm{H}$ NMR $\left(500 \mathrm{MHz}, \mathrm{C}_{6} \mathrm{D}_{6}\right): \delta 7.71(\mathrm{t}, J=11.0 \mathrm{~Hz}, 6 \mathrm{H}, \mathrm{Ar}), 7.68$ (dd, $\left.J=5.5,2.0 \mathrm{~Hz}, 1 \mathrm{H}, \mathrm{Ar}\right), 7.14-6.98$ (m, 11H, Ar), 6.57 (d, $J=9.5 \mathrm{~Hz}, 1 \mathrm{H}, \mathrm{Ar}), 6.01$ (d, $J=10.5 \mathrm{~Hz}, 1 \mathrm{H}, \mathrm{Ar}), 3.90$ (d, J = 12.5 Hz, $\left.2 \mathrm{H}, \mathrm{CH}_{2} \mathrm{Ar}\right), 3.37$ (s, 3H, OMe), 2.48-2.30 (m, 2H, $\left.\mathrm{PCH}_{2}\right), 1.44(\mathrm{~s}, 9 \mathrm{H}, t-\mathrm{Bu}), 1.27$ (s, 9H, $t$-Bu), 0.96 (s, 3H, Me), 0.94 (s, 3H, Me). ${ }^{13} \mathrm{C}\left\{{ }^{1} \mathrm{H}\right\}$ NMR (125 MHz, $\left.\mathrm{C}_{6} \mathrm{D}_{6}\right): \delta 167.1$ (Ar), 161.7 (Ar), 157.7 (d, $J=26.5 \mathrm{~Hz}, \mathrm{Ar}$ ), 157.3 (d, $J=9.5 \mathrm{~Hz}, \mathrm{Ar}$ ), 153.5 (Ar), 135.9 (Ar), 135.4 (Ar), 130126 (overlapping with residual solvent) 117.9 (d, $J=11.4 \mathrm{~Hz}, \mathrm{Ar}$ ), 115.0 (Ar), 104.6 (Ar), 55.8 (OMe), 50.7 (d, $\left.J=28.4 \mathrm{~Hz}, \mathrm{CH}_{2} \mathrm{Ar}\right), 48.2$ (d, $\left.J=53.0 \mathrm{~Hz}, \mathrm{PCH}_{2}\right), 43.3\left(\mathrm{CMe}_{2}\right), 38.4\left(C \mathrm{Me}_{3}\right)$, $35.6\left(\mathrm{CMe}_{2}\right), 35.0\left(\mathrm{CMe}_{3}\right), 34.7(t-\mathrm{Bu}), 31.2(t-\mathrm{Bu}), 29.0(\mathrm{~d}, J=10.4 \mathrm{~Hz}, \mathrm{CMe})_{2}$. 
${ }^{31} \mathrm{P}\left\{{ }^{1} \mathrm{H}\right\}$ NMR $\left(202 \mathrm{MHz}, \mathrm{C}_{6} \mathrm{D}_{6}\right)$

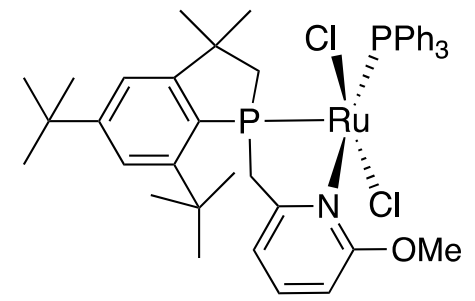

${ }^{31} \mathrm{P}\left\{{ }^{1} \mathrm{H}\right\}$ NMR $\left(202 \mathrm{MHz}, \mathrm{C}_{6} \mathrm{D}_{6}\right)$ : Zoom in on doublets
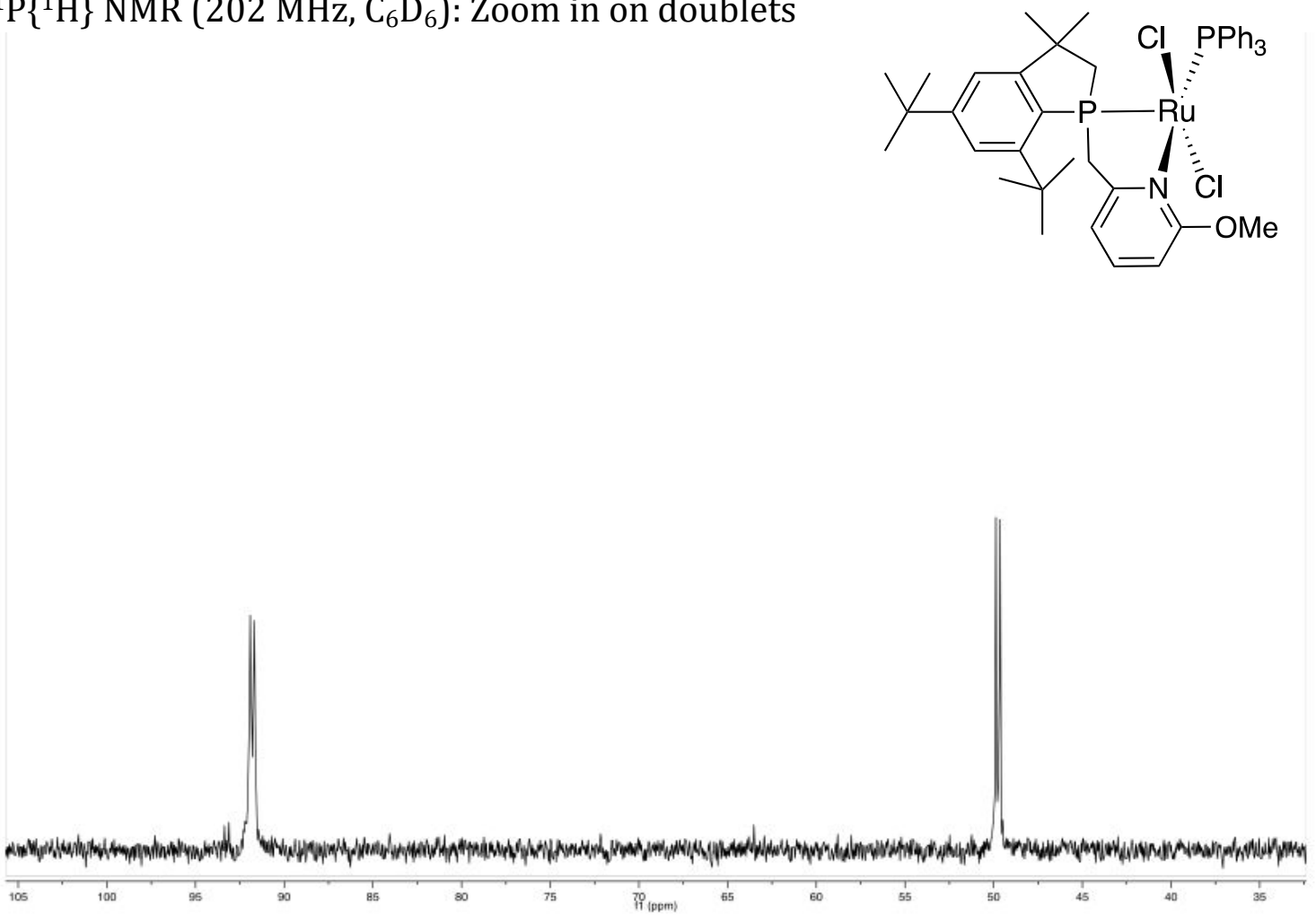
${ }^{1} \mathrm{H}$ NMR (500 MHz, $\mathrm{C}_{6} \mathrm{D}_{6}$ )
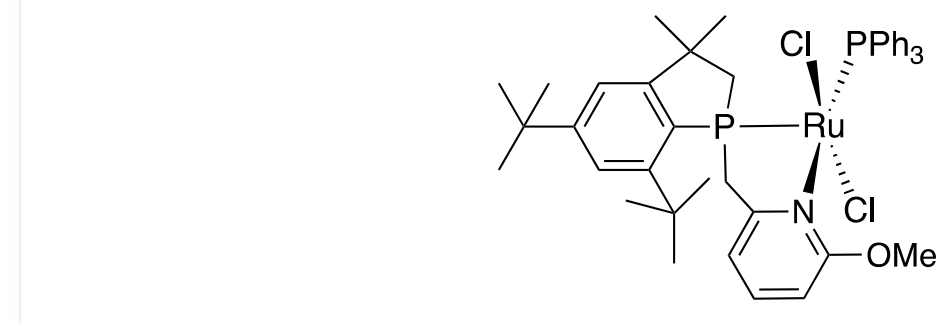

${ }^{13} \mathrm{C}\left\{{ }^{1} \mathrm{H}\right\}$ NMR $\left(125 \mathrm{MHz}, \mathrm{C}_{6} \mathrm{D}_{6}\right)$

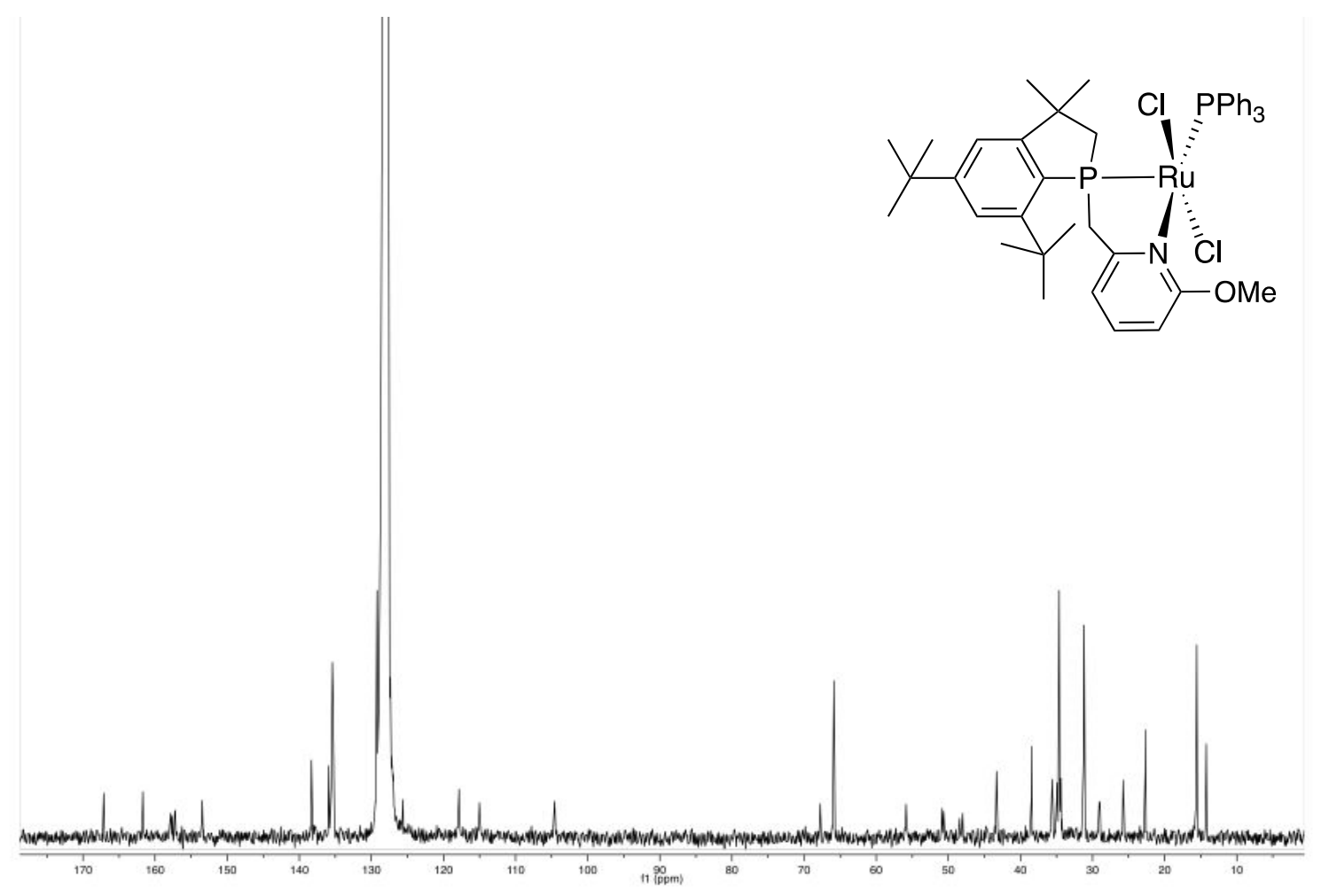


${ }^{13} \mathrm{C}\left\{{ }^{1} \mathrm{H}\right\}$ NMR $\left(125 \mathrm{MHz}, \mathrm{C}_{6} \mathrm{D}_{6}\right)$ : Zoomed in on Aryl Region

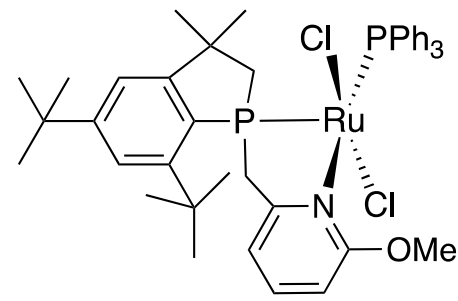

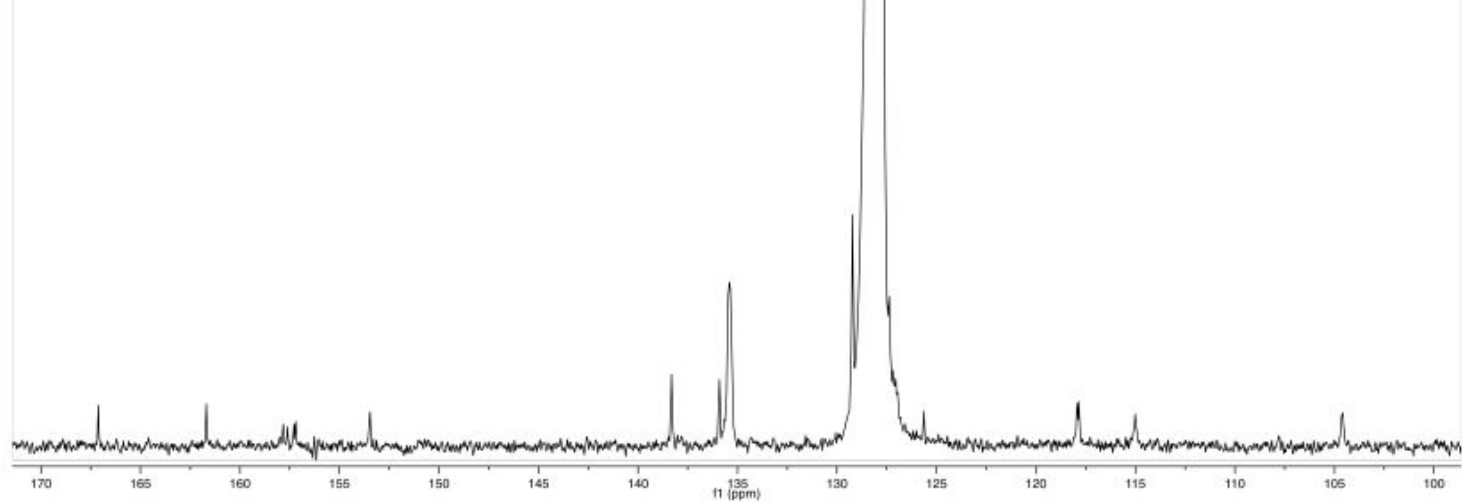

${ }^{13} \mathrm{C}\left\{{ }^{1} \mathrm{H}\right\}$ NMR $\left(125 \mathrm{MHz}, \mathrm{C}_{6} \mathrm{D}_{6}\right)$ : Zoomed in on Alkyl Region
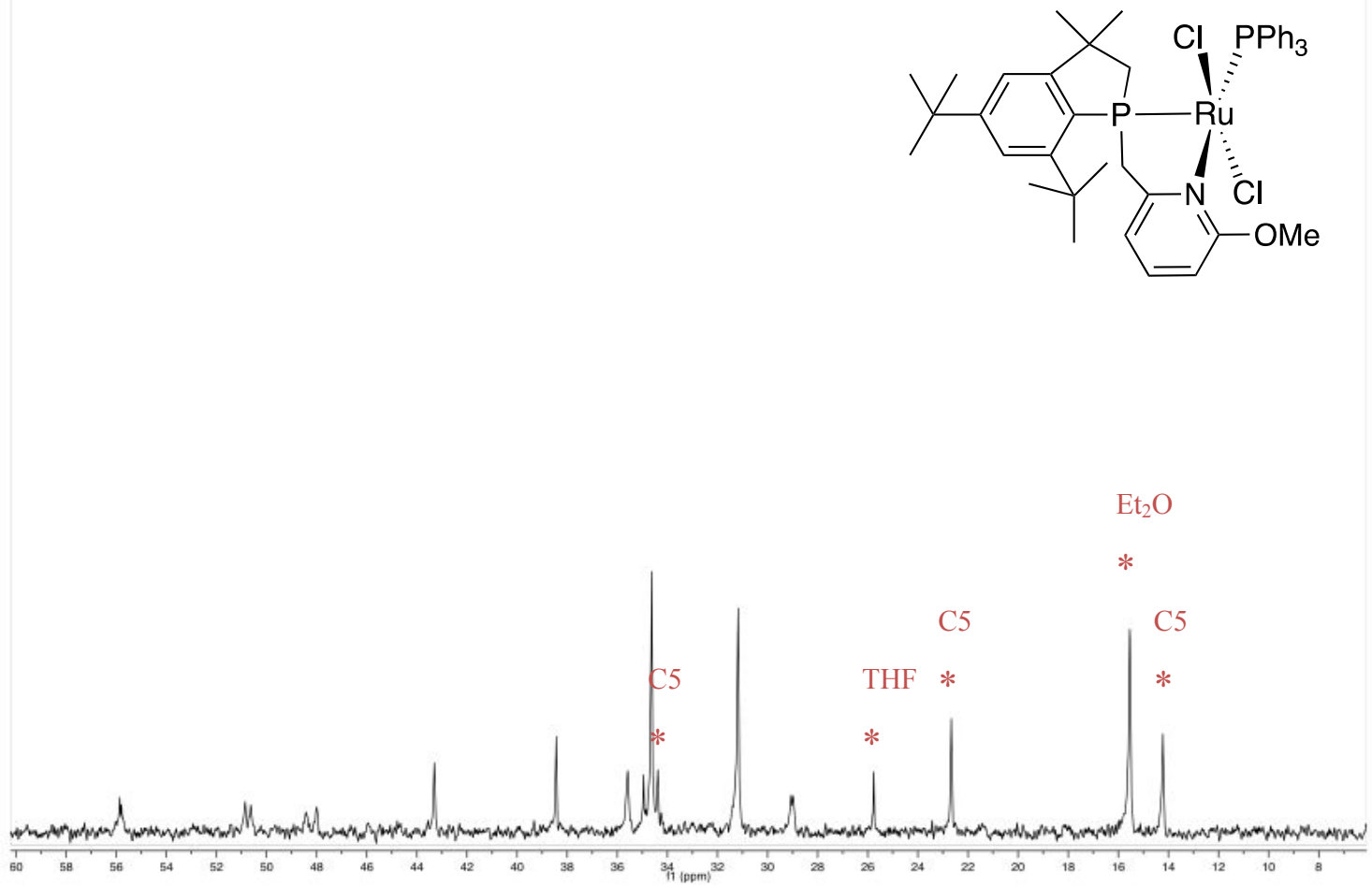
Synthesis of $4 \mathbf{e}\left(\mathrm{R}=\mathrm{Ar}-m-\mathrm{NO}_{2}\right)$

$\mathrm{RuCl}_{2}\left(\mathrm{PPh}_{3}\right)_{3}(197 \mathrm{mg}, 0.205 \mathrm{mmol})$ was loaded into a vial, and treated with a solution of 2e $(100 \mathrm{mg}, 0.205 \mathrm{mmol})$ in $2 \mathrm{~mL}$ of benzene at room temperature. The dark reaction mixture was transferred to a J-Young tube and monitored by ${ }^{31} \mathrm{P}\left\{{ }^{1} \mathrm{H}\right\}$ NMR spectroscopy. After heating at $80{ }^{\circ} \mathrm{C}$ for $72 \mathrm{~h}$, the reaction mixture turned dark green, and the ${ }^{31} \mathrm{P}\left\{{ }^{1} \mathrm{H}\right\}$ NMR spectrum showed two doublets at $90.3 \mathrm{ppm}$ and $48.6 \mathrm{ppm}$. The mixture was subsequently filtered through Celite and the filtrate was concentrated under vacuum. The dark green residue was recrystallized from a $5 \mathrm{~mL}$ solution of THF layered with pentane at $-35{ }^{\circ} \mathrm{C}$ (185 mg, $0.201 \mathrm{mmol}, 98 \%$ yield). Crystals suitable for X-ray crystallography were collected by slow diffusion of pentane vapors into a concentrated THF solution.

Anal. Calcd. for $\mathrm{C}_{48} \mathrm{H}_{52} \mathrm{Cl}_{2} \mathrm{~N}_{2} \mathrm{O}_{2} \mathrm{P}_{2} \mathrm{Ru}$ was consistently low (3 attempts) in carbon, for example: $\mathrm{C}_{48} \mathrm{H}_{52} \mathrm{Cl}_{2} \mathrm{~N}_{2} \mathrm{O}_{2} \mathrm{P}_{2} \mathrm{Ru}$ : C, 62.47; H, 5.68; N, 3.04. Found: C, 61.83; H, 5.69; N, 2.69. HRMS: $\mathrm{m} / \mathrm{z}$ was calcd for $\mathrm{C}_{48} \mathrm{H}_{52} \mathrm{Cl}_{2} \mathrm{~N}_{2} \mathrm{O}_{2} \mathrm{P}_{2} \mathrm{Ru}[\mathrm{M}]^{+}$: 922.1925. Found: 922.1906. ${ }^{31} \mathrm{P}\left\{{ }^{1} \mathrm{H}\right\}$ NMR ( $\mathrm{CDCl}_{3}$ ): $\delta 90.3$ (d, $\left.J=36.4 \mathrm{~Hz}, \mathrm{P}-\mathrm{Mes}^{*}\right), 48.6\left(\mathrm{~d}, J=36.4 \mathrm{~Hz}, \mathrm{PPh}_{3}\right) .{ }^{1} \mathrm{H}$ NMR $\left(\mathrm{CDCl}_{3}\right): \delta$ 8.57 (s, 1H, Ar), 8.11 (d, J = 7.0 Hz, 1H, Ar), 8.03 (d, J = 9.5 Hz, 1H, Ar), 7.87 (t, J = 8.0 Hz, 1H, $\mathrm{Ar}$ ), 7.58-7.54 (m, 3H, Ar), $7.27(\mathrm{~m}, J=7.0 \mathrm{~Hz}, 9 \mathrm{H}, \mathrm{Ar}), 7.08(\mathrm{t}, J=6.5 \mathrm{~Hz}, 6 \mathrm{H}, \mathrm{Ar}), 6.95(\mathrm{~s}, 1 \mathrm{H}$, Mes*), 6.89 (t, $J=8.0 \mathrm{~Hz}, 1 \mathrm{H}, \mathrm{Ar}$ ), 4.25 (overlapping $\mathrm{m}, J=16.5,10 \mathrm{~Hz}, 2 \mathrm{H}, \mathrm{PCH}_{2} \mathrm{~N}$ ), $2.34(\mathrm{dd}$, $\left.J=16.5,5.0 \mathrm{~Hz}, 1 \mathrm{H}, \mathrm{PCH}_{2}\right), 1.90-1.81\left(\mathrm{~m}, 1 \mathrm{H}, \mathrm{PCH}_{2}\right), 1.35(\mathrm{~s}, 9 \mathrm{H}, t-\mathrm{Bu}), 1.15(\mathrm{~s}, 9 \mathrm{H}, t-\mathrm{Bu})$, 1.12 (s, 3H, C(Me) $\left.)_{2}\right), 0.78$ (s, 3H, C(Me) ${ }_{2}$ ). ${ }^{13} \mathrm{C}\left\{{ }^{1} \mathrm{H}\right\} \mathrm{NMR}\left(125 \mathrm{MHz}, \mathrm{CDCl}_{3}\right), \delta: 164.1$ (Ar), 160.8 (Ar), 157.5 (d, $J=21.3 \mathrm{~Hz}, \mathrm{Ar}$ ), 156.6 (d, $J=7.5 \mathrm{~Hz}, \mathrm{Ar}$ ), $153.7(\mathrm{Ar}), 148.8$ (Ar), 142.6 (Ar), 136.8 (Ar), 134.9 (d, $J=8.8 \mathrm{~Hz}, \mathrm{Ar}$ ), 133.8 (d, $J=42.5 \mathrm{~Hz}, \mathrm{Ar}$ ), 131.9 (Ar), 131.4 (Ar), 129.2 (Ar), 127.6 (d, $J=10.0 \mathrm{~Hz}, \mathrm{Ar}$ ), 126.8 (d, $J=10.0 \mathrm{~Hz}, \mathrm{Ar}$ ), 126.4 (Ar), 123.9 (Ar), 123.8 (Ar), 123.2 (Ar), 121.6 (d, $J=10.0 \mathrm{~Hz}, \mathrm{Ar}$ ), 118.1 (d, $J=10.0 \mathrm{~Hz}, \mathrm{Ar}), 50.9$ (d, $J=22.5 \mathrm{~Hz}$, benzylic), 45.9 (d, $J=42.5 \mathrm{~Hz}$, Phospholane $\left.\mathrm{PCH}_{2}\right), 42.7\left(\mathrm{CMe}_{2}\right), 37.7\left(\mathrm{CMe}_{3}\right), 35.3\left(\mathrm{CMe}_{2}\right)$, $35.0\left(\mathrm{CMe}_{3}\right), 34.0(t-\mathrm{Bu}), 31.0(t-\mathrm{Bu}), 28.8(\mathrm{~d}, J=10 \mathrm{~Hz}, \mathrm{CMe})_{2}$ ). 
${ }^{31} \mathrm{P}\left\{{ }^{1} \mathrm{H}\right\} \mathrm{NMR}\left(\mathrm{CDCl}_{3}\right)$

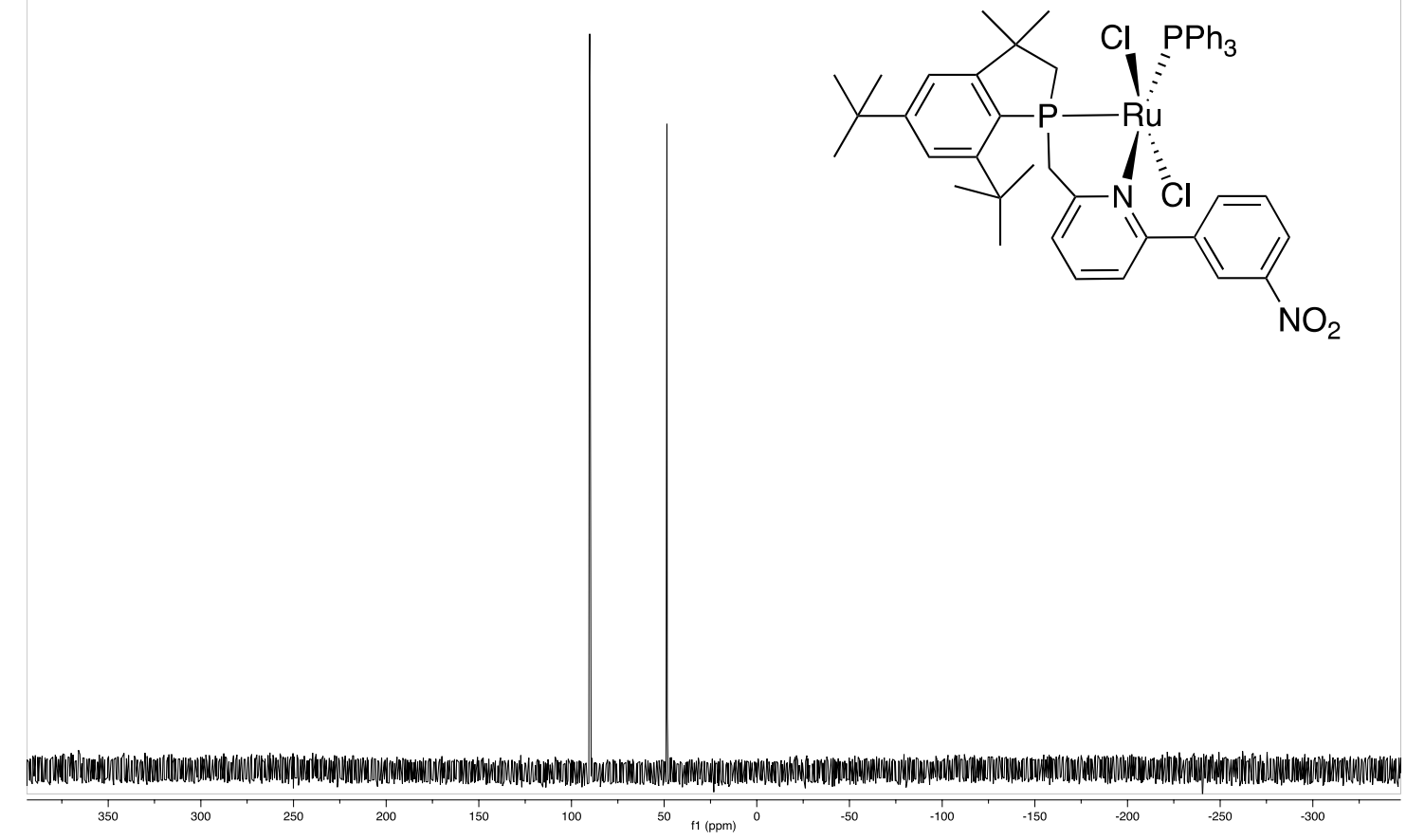

${ }^{31} \mathrm{P}\left\{{ }^{1} \mathrm{H}\right\} \mathrm{NMR}\left(\mathrm{CDCl}_{3}\right):$ Zoomed in on doublets
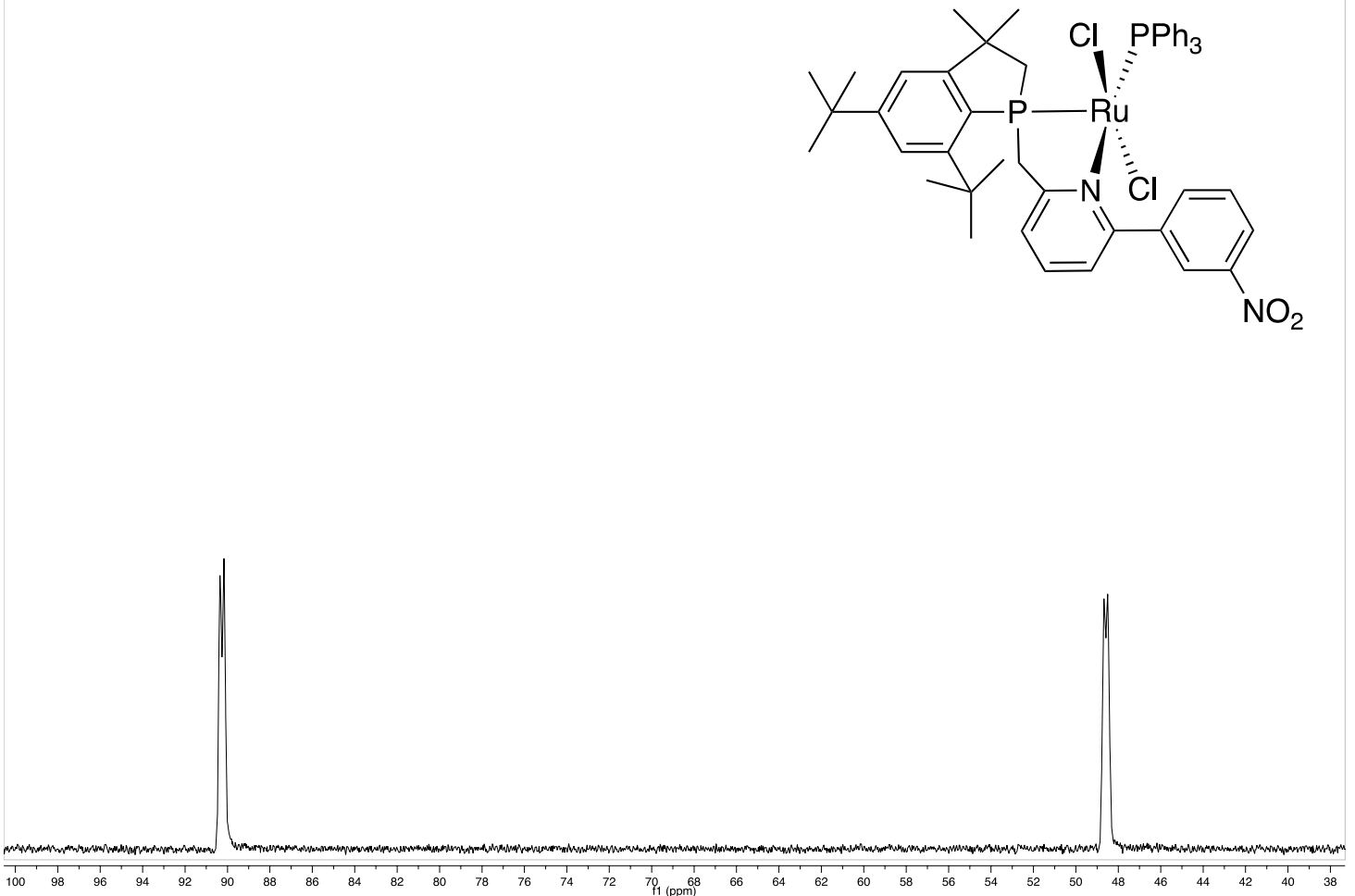


\section{${ }^{1} \mathrm{H}$ NMR $\left(\mathrm{CDCl}_{3}\right)$}

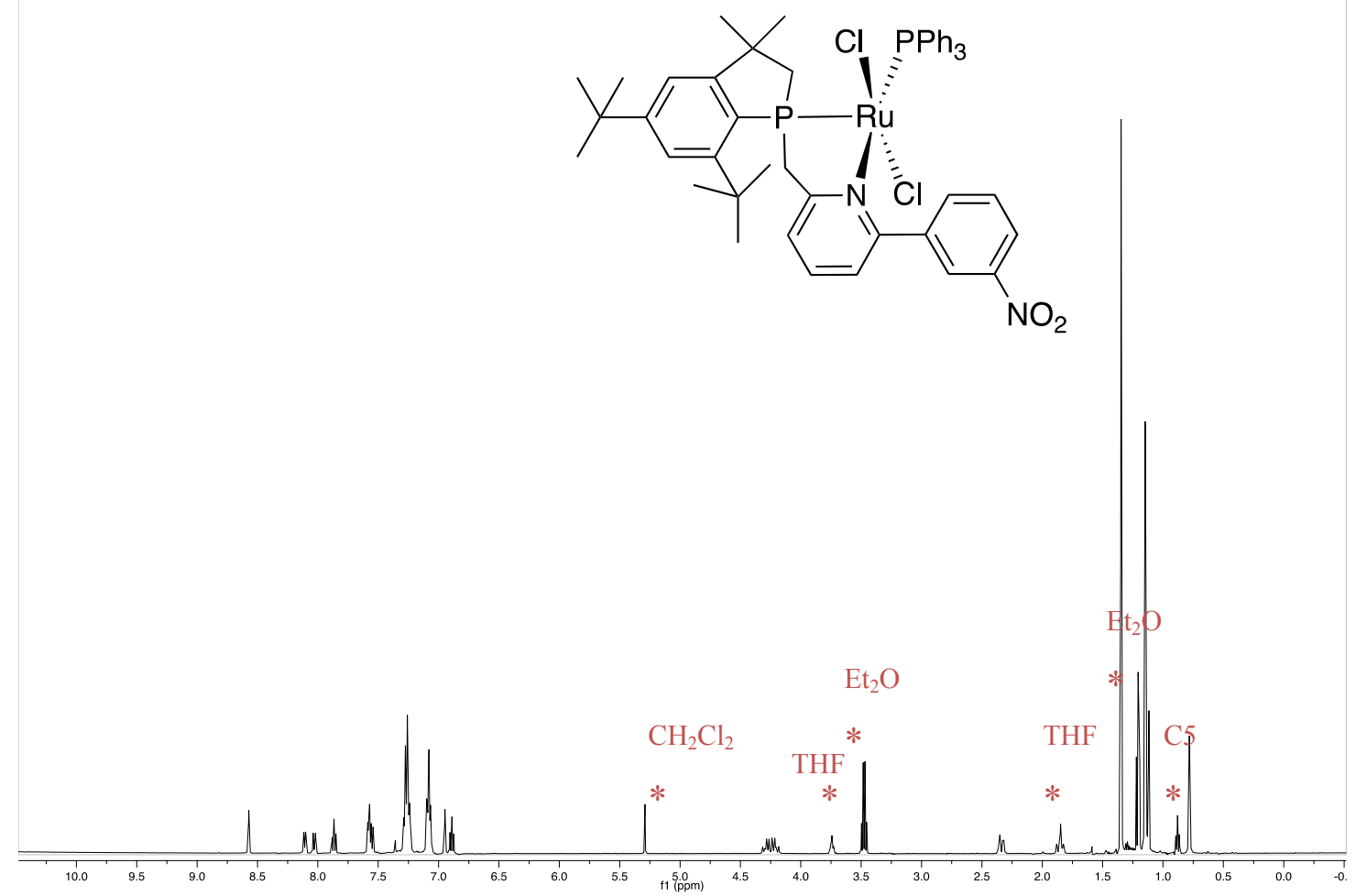

${ }^{1} \mathrm{H}$ NMR $\left(\mathrm{CDCl}_{3}\right)$ : Benzylic Protons
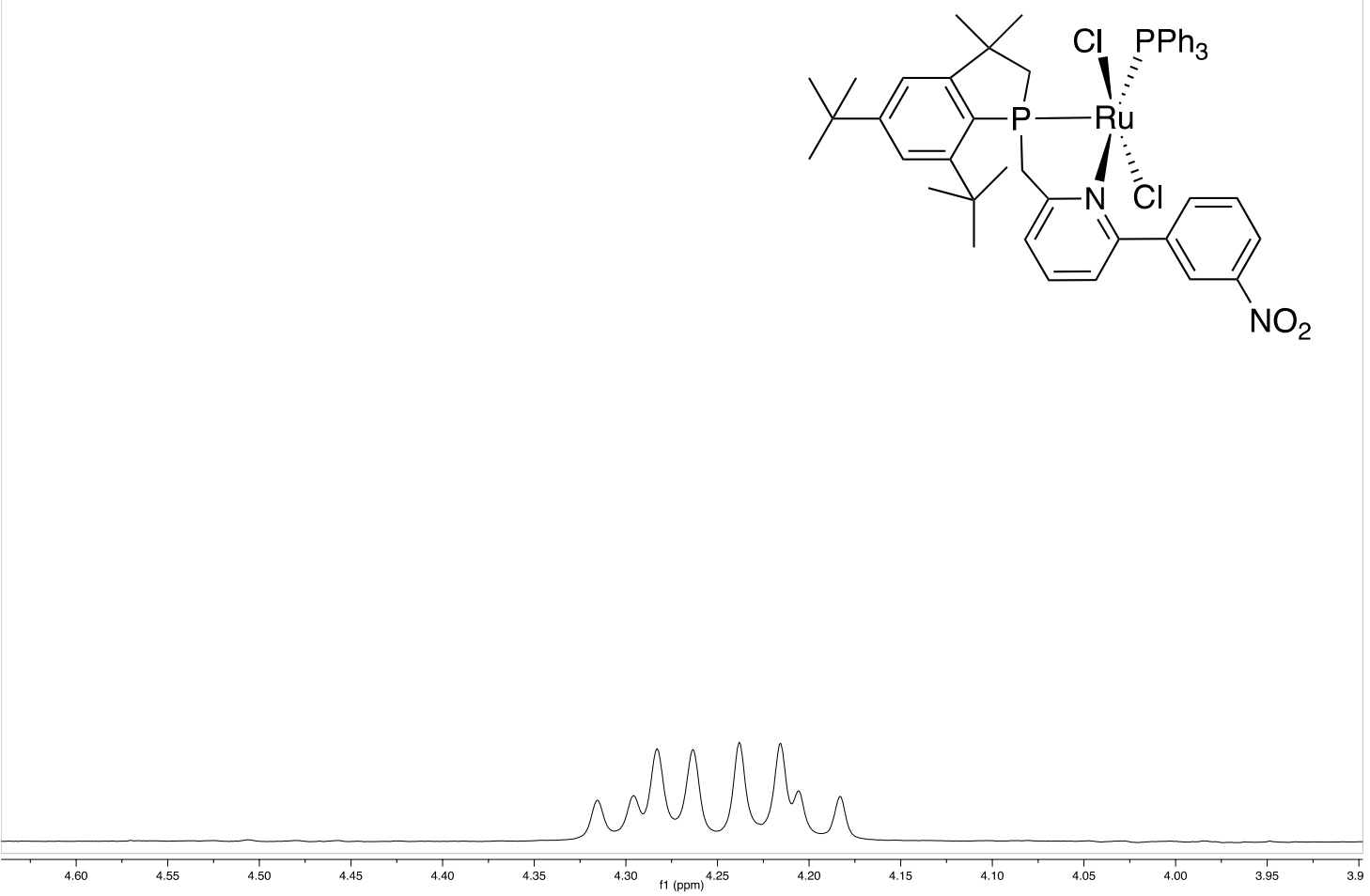
${ }^{1} \mathrm{H}$ NMR $\left(\mathrm{CDCl}_{3}\right)$ : Phospholane Protons
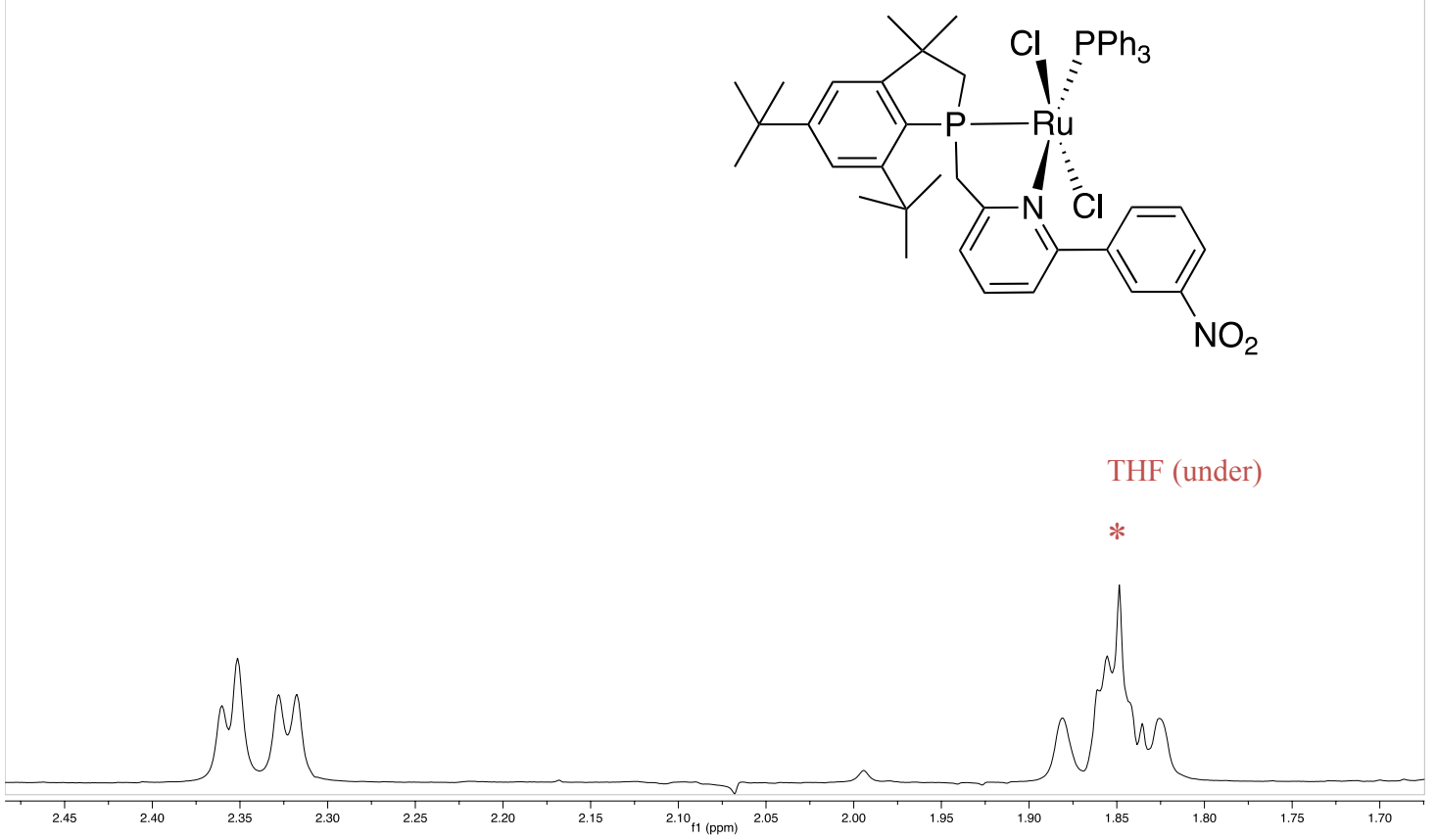

${ }^{13} \mathrm{C}\left\{{ }^{1} \mathrm{H}\right\}$ NMR $\left(\mathrm{CDCl}_{3}\right)$

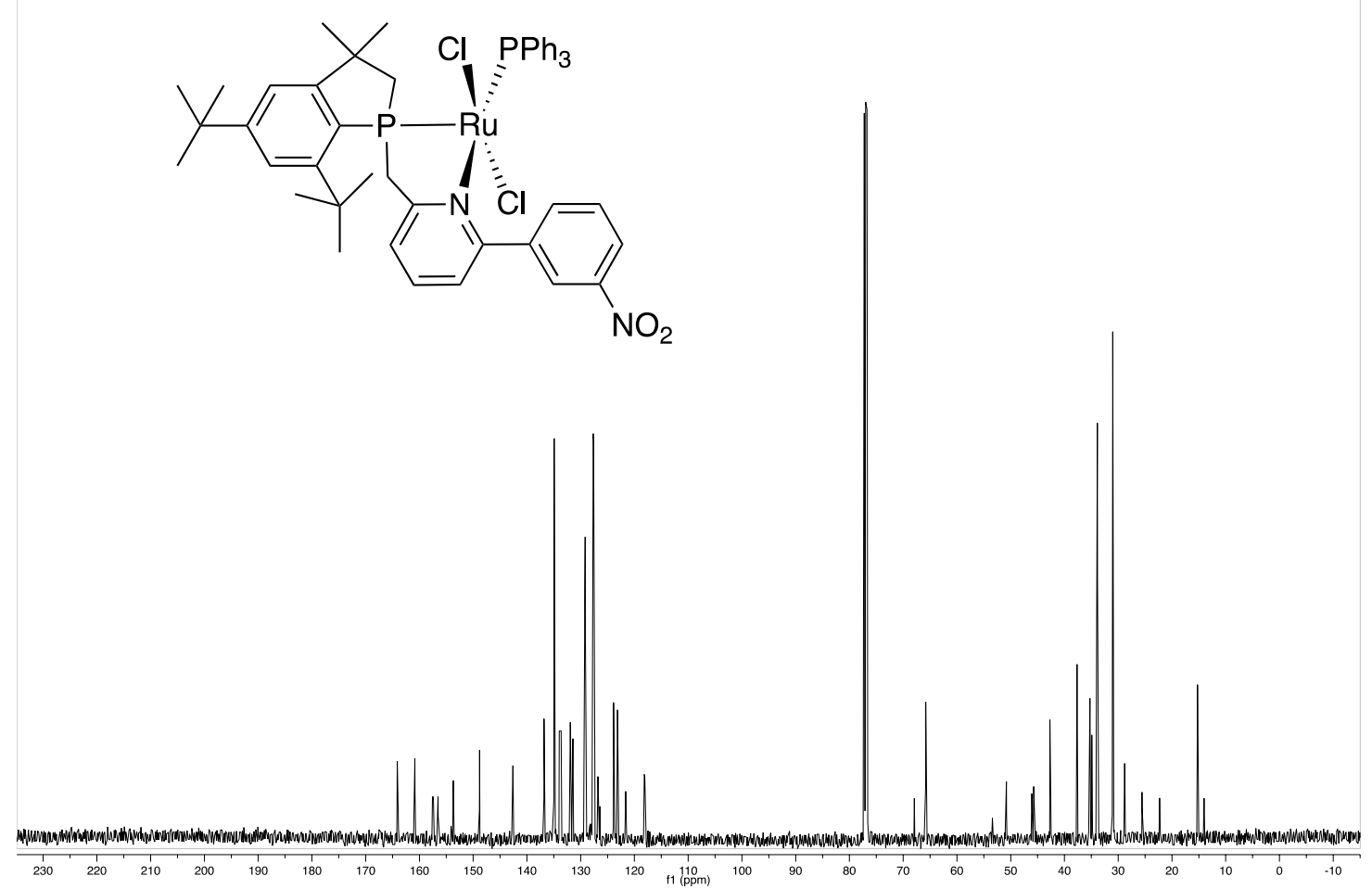


${ }^{13} \mathrm{C}\left\{{ }^{1} \mathrm{H}\right\}$ NMR $\left(\mathrm{CDCl}_{3}\right)$ : Aryl Region
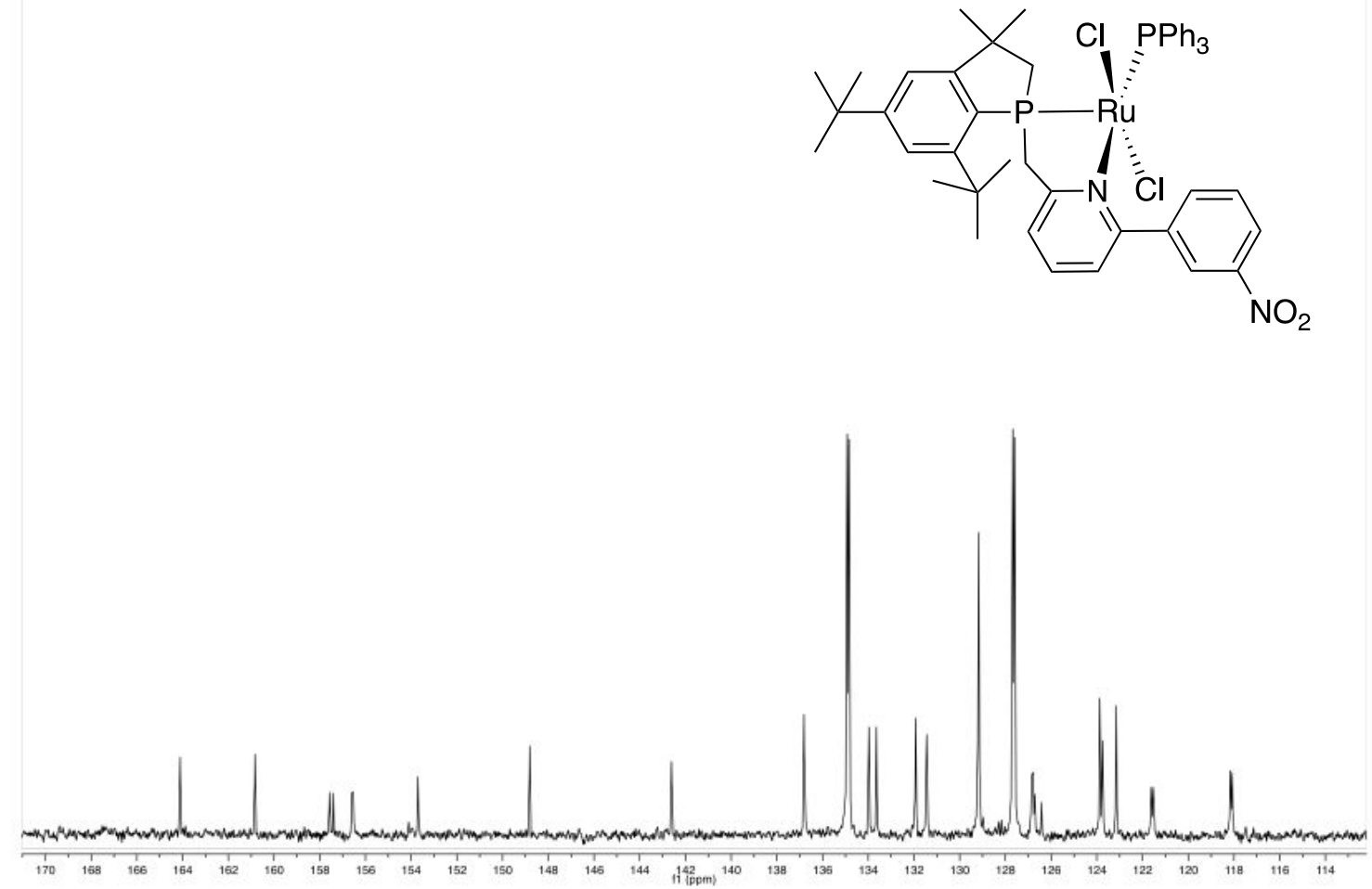

${ }^{13} \mathrm{C}\left\{{ }^{1} \mathrm{H}\right\}$ NMR (CDCl 3 ): Alkyl Region

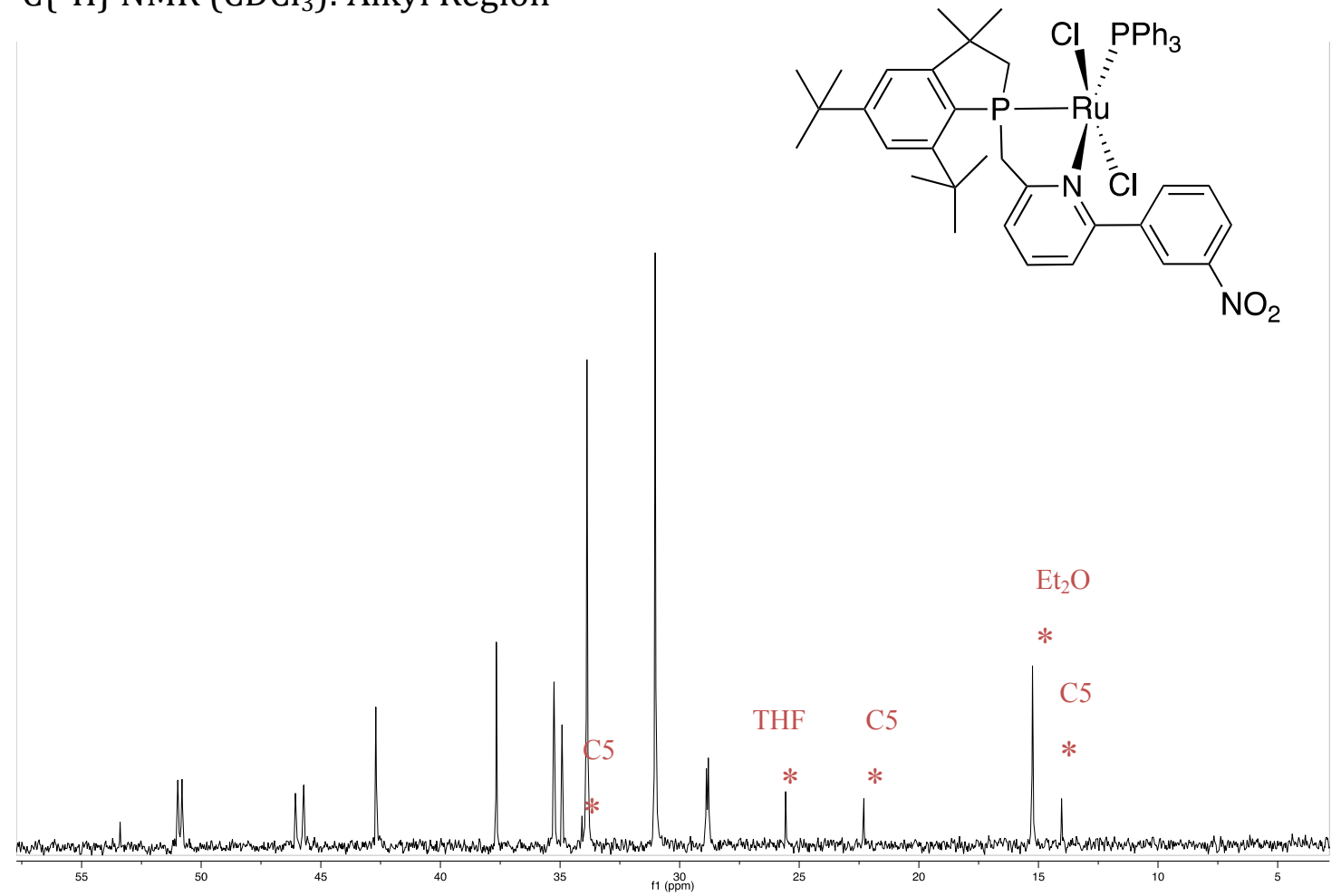




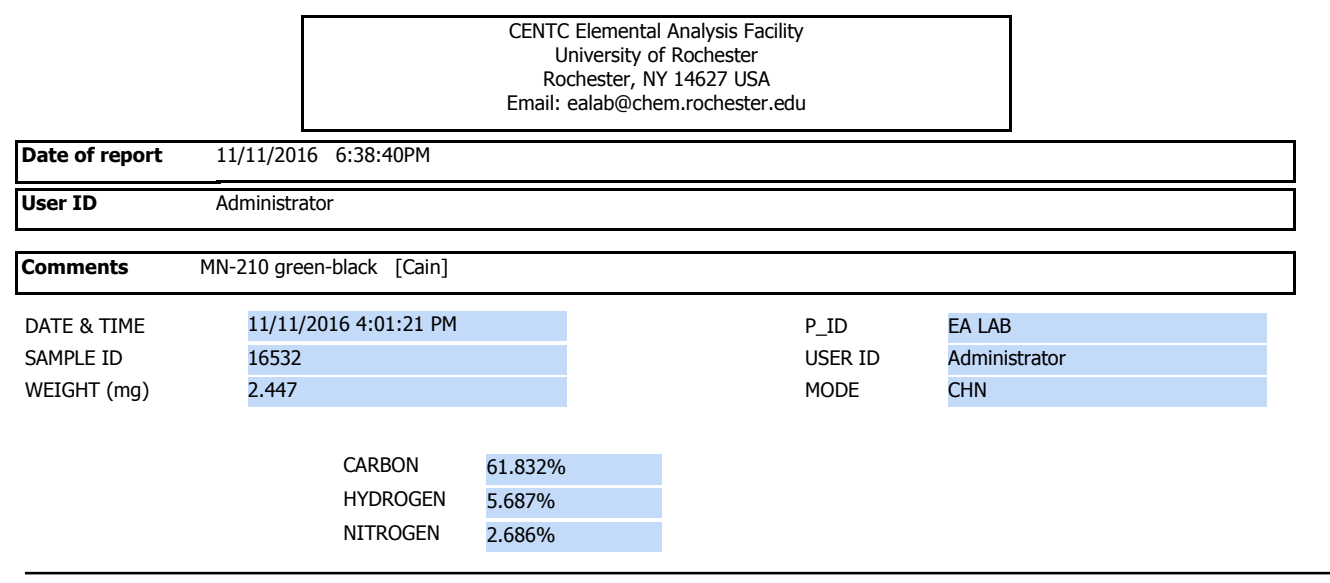

Special Handling

The sample was transferred under argon and was combusted in a tin capsule that was crimp-sealed with a die apparatus.

Acknowledgment

Analytical data were obtained from the CENTC Elemental Analysis Facility at the University of Rochester, funded by NSF CHE-0650456.

Instrumentation

Microanalysis samples were weighed with a PerkinElmer Model AD-6 Autobalance and their compositions were determined with a PerkinElmer 2400 Series II Analyzer. Air-sensitive samples were handled in a VAC Atmospheres glovebox.

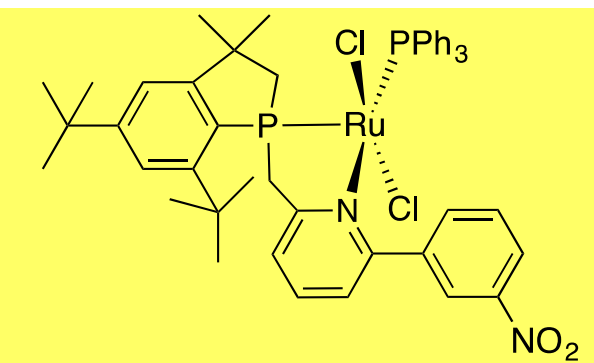

Chemical Formula: $\mathrm{C}_{48} \mathrm{H}_{52} \mathrm{Cl}_{2} \mathrm{~N}_{2} \mathrm{O}_{2} \mathrm{P}_{2} \mathrm{Ru}$

Exact Mass: 922.1925

Molecular Weight: 922.8735

Elemental Analysis:

C, 62.47; H, 5.68; Cl, 7.68; N, 3.04;

$\mathrm{O}, 3.47 ; \mathrm{P}, 6.71 ; \mathrm{Ru}, 10.95$

Page 1 of 1 


\section{Synthesis of $5 \mathbf{a}(\mathrm{R}=\mathrm{H})$}

$\mathrm{RuCl}_{2}\left(\mathrm{PPh}_{3}\right)_{3}$ (55 mg, $0.149 \mathrm{mmol}, 1.0$ equiv.) was loaded into a vial, and treated with a solution of $2 \mathrm{a}$ (144 mg, $0.149 \mathrm{mmol}, 1.0$ equiv.) in $1 \mathrm{~mL}$ benzene at room temperature. The dark reaction mixture was transferred to a J-Young tube and monitored by ${ }^{31} \mathrm{P}\left\{{ }^{1} \mathrm{H}\right\}$ NMR spectroscopy. After heating at $80{ }^{\circ} \mathrm{C}$ for $72 \mathrm{~h}$, the solution turned dark red, and the ${ }^{31} \mathrm{P}\left\{{ }^{1} \mathrm{H}\right\}$ NMR spectrum showed two doublets at $66.8 \mathrm{ppm}$ and $64.0 \mathrm{ppm}$. The mixture was subsequently filtered through a Celite plug using $\mathrm{Et}_{2} \mathrm{O}$ and the filtrate was concentrated under vacuum. The crude product was recrystallized from a concentrated solution of benzene at $25{ }^{\circ} \mathrm{C}$ to give a red solid $(50 \mathrm{mg}, 0.062$ mmol, $42 \%$ yield). Crystals suitable for X-ray crystallography were collected by slow diffusion of pentane vapors into a concentrated THF solution.

Anal. Calcd. for $\mathrm{C}_{42} \mathrm{H}_{49} \mathrm{Cl}_{2} \mathrm{NP}_{2} \mathrm{Ru}$ : C, 62.92; H, 6.16; N, 1.75. Found: C, 63.31; H, 6.12; N, 1.37 . HRMS: $\mathrm{m} / \mathrm{z}$ was calcd for $\mathrm{C}_{42} \mathrm{H}_{49} \mathrm{ClNP}_{2} \mathrm{Ru}^{+}\left[\mathrm{M}-\left(\mathrm{Cl}^{-}\right)\right]^{+}:$766.2067. Found 766.2077. ${ }^{31} \mathrm{P}\left\{{ }^{1} \mathrm{H}\right\}$ NMR (202 MHz, $\left.\mathrm{CDCl}_{3}\right): \delta 66.8\left(\mathrm{~d}, J=39.3 \mathrm{~Hz}, \mathrm{PPh}_{3}\right), 64.0\left(\mathrm{~d}, J=39.3 \mathrm{~Hz}, \mathrm{CH}_{2} \mathrm{P}\right) .{ }^{1} \mathrm{H}$ NMR $\left(500 \mathrm{MHz}, \mathrm{CDCl}_{3}\right): \delta 9.31(\mathrm{dd}, J=6.1,1.8 \mathrm{~Hz}, 1 \mathrm{H}, \mathrm{Ar}), 7.76-7.82(\mathrm{~m}, 5 \mathrm{H}, \mathrm{Ar}), 7.42(\mathrm{dd}, J=4.8$, $1.8 \mathrm{~Hz}, 1 \mathrm{H}, \mathrm{Ar}), 7.27-7.20$ (m, 5H, Ar), 7.19-7.13 (m, 6H, Ar), 7.10 (d, J=1.8 Hz, 1H, Mes*), 7.04 (d, $J=7.1 \mathrm{~Hz}, 1 \mathrm{H}, \mathrm{Ar}), 6.43$ (t, $J=7.1 \mathrm{~Hz}, 1 \mathrm{H}, \mathrm{Ar}), 3.69$ (dd, $J=18.4,11.4 \mathrm{~Hz}, 1 \mathrm{H}$, $\mathrm{CH}_{2} \mathrm{Ar}$ ), 3.44 (dd, $\left.J=18.4,8.5 \mathrm{~Hz}, 1 \mathrm{H}, \mathrm{CH}_{2} \mathrm{Ar}\right), 2.22$ (dd, $\left.J=15.3,7.8 \mathrm{~Hz}, 1 \mathrm{H}, \mathrm{PCH}_{2}\right), 1.30$ (s, $9 \mathrm{H}, t-\mathrm{Bu}), 1.26(\mathrm{~s}, 3 \mathrm{H}, \mathrm{Me}), 1.25(\mathrm{~s}, 3 \mathrm{H}, \mathrm{Me}), 0.86(\mathrm{~d}, J=1.6 \mathrm{~Hz}, 9 \mathrm{H}, t-\mathrm{Bu}), 0.73$ (dd, $J=15.2$, $\left.2.2 \mathrm{~Hz}, 1 \mathrm{H}, \mathrm{PCH}_{2}\right) .{ }^{13} \mathrm{C}\left\{{ }^{1} \mathrm{H}\right\} \mathrm{NMR}\left(125 \mathrm{MHz}, \mathrm{CDCl}_{3}\right): \delta 164.7$ (Ar), 157.0 (Ar), 156.2 (d, $J=$ 14.2 Hz, Ar), 154.2 (Ar), 152.2 (d, $J=10.6 \mathrm{~Hz}, \mathrm{Ar}), 134.8$ (d, $J=22.3 \mathrm{~Hz}, \mathrm{Ar}), 134.6$ (Ar), 133.9 (d, $J=9.5 \mathrm{~Hz}, \mathrm{Ar}), 129.0$ (Ar), 127.6 (d, $J=10.1 \mathrm{~Hz}, \mathrm{Ar}), 124.6$ (d, $J=8.2 \mathrm{~Hz}, \mathrm{Ar}), 121.9$ (Ar), 121.7 (d, $J=11.4 \mathrm{~Hz}, \mathrm{Ar}), 118.8$ (d, $J=8.2 \mathrm{~Hz}, \mathrm{Ar}), 46.8$ (d, $\left.J=20.0 \mathrm{~Hz}, \mathrm{CH}_{2} \mathrm{Ar}\right), 43.6$ (d, $\left.J=5.5 \mathrm{~Hz}, C \mathrm{Me}_{2}\right), 39.4\left(\mathrm{~d}, J=31.3 \mathrm{~Hz}, \mathrm{PCH}_{2}\right), 35.0\left(C \mathrm{Me}_{3}\right), 30.4\left(C \mathrm{Me}_{3}\right), 32.8(t-\mathrm{Bu}), 31.7$ (d, $\left.J=9.6 \mathrm{~Hz}, \mathrm{CMe} e_{2}\right), 31.2(t-\mathrm{Bu}), 30.5\left(\mathrm{~d}, J=5.3 \mathrm{~Hz}, \mathrm{CMe} e_{2}\right)$. 
${ }^{31} \mathrm{P}\left\{{ }^{1} \mathrm{H}\right\}$ NMR $\left(202 \mathrm{MHz}, \mathrm{CDCl}_{3}\right)$

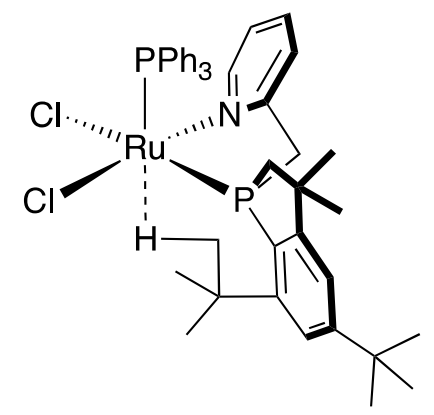

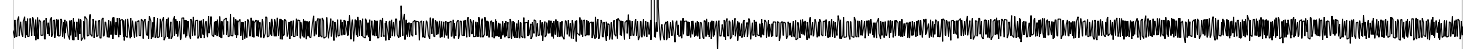

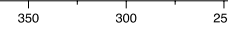

$200 \quad 150$

${ }^{31} \mathrm{P}\left\{{ }^{1} \mathrm{H}\right\}$ NMR $\left(202 \mathrm{MHz}, \mathrm{CDCl}_{3}\right)$ : Zoomed in on doublets

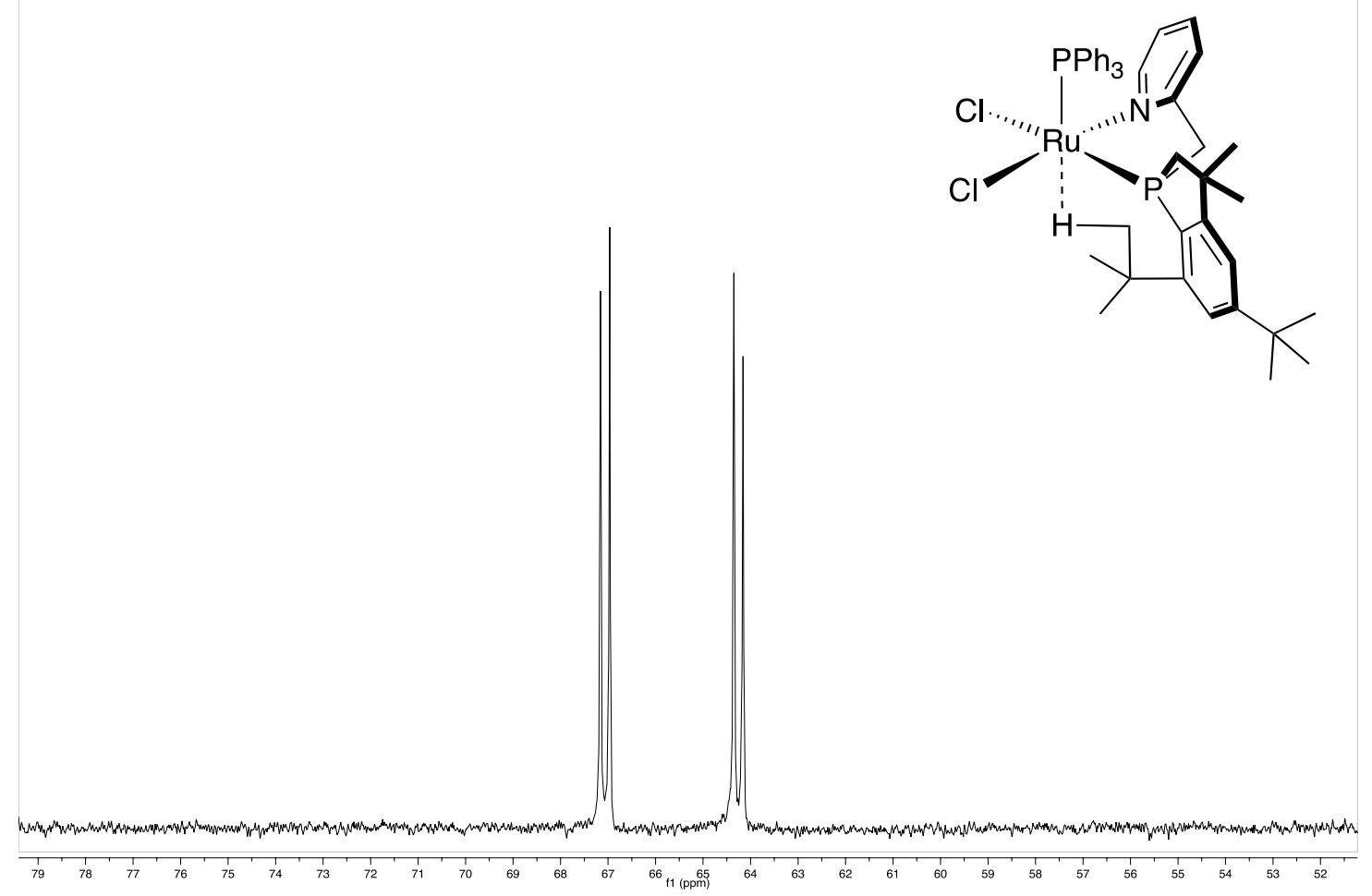


${ }^{1} \mathrm{H}$ NMR $\left(500 \mathrm{MHz}, \mathrm{CDCl}_{3}\right)$

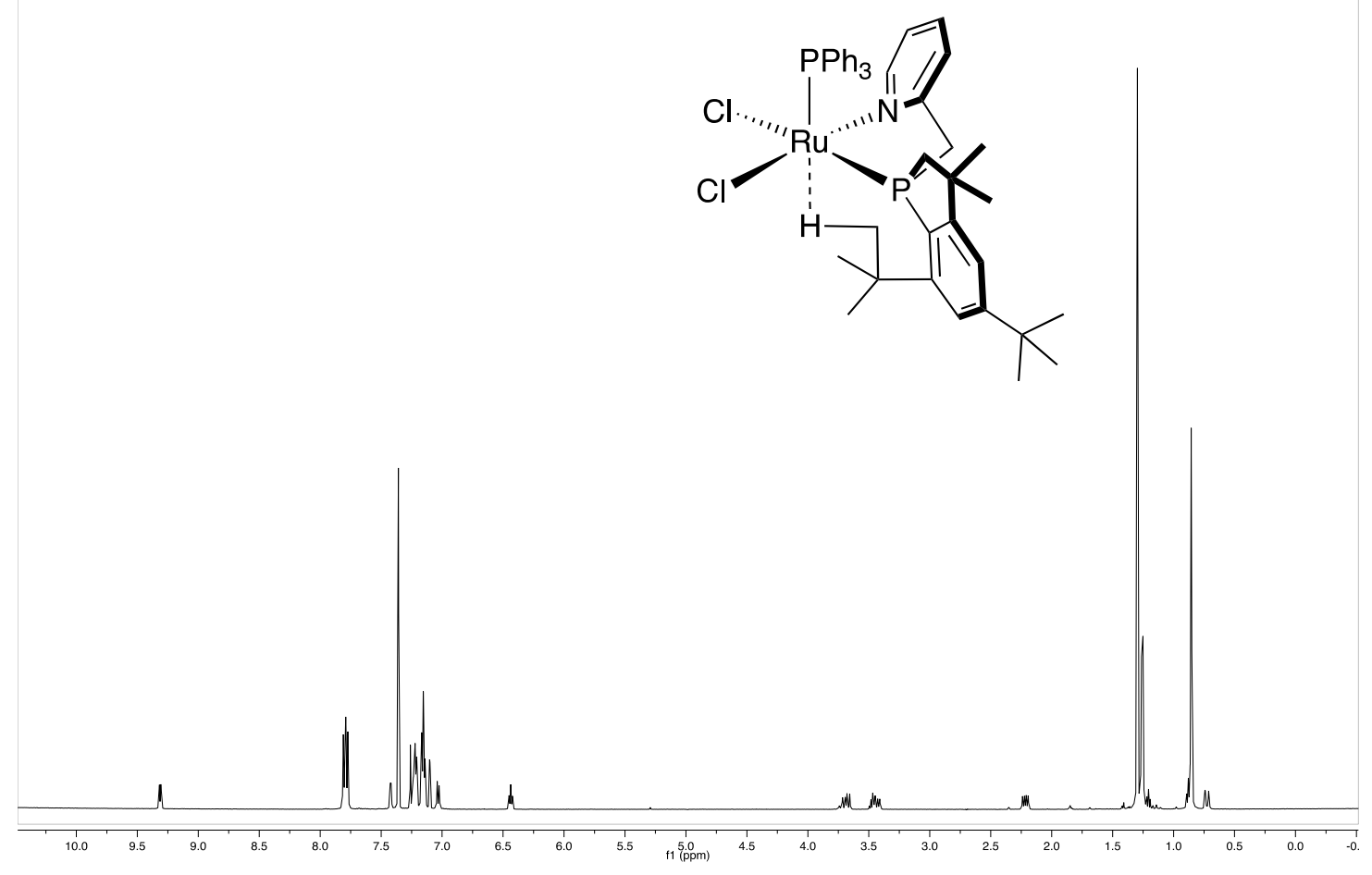

${ }^{1} \mathrm{H}$ NMR $\left(500 \mathrm{MHz}, \mathrm{CDCl}_{3}\right)$ : Zoomed in on benzylic protons
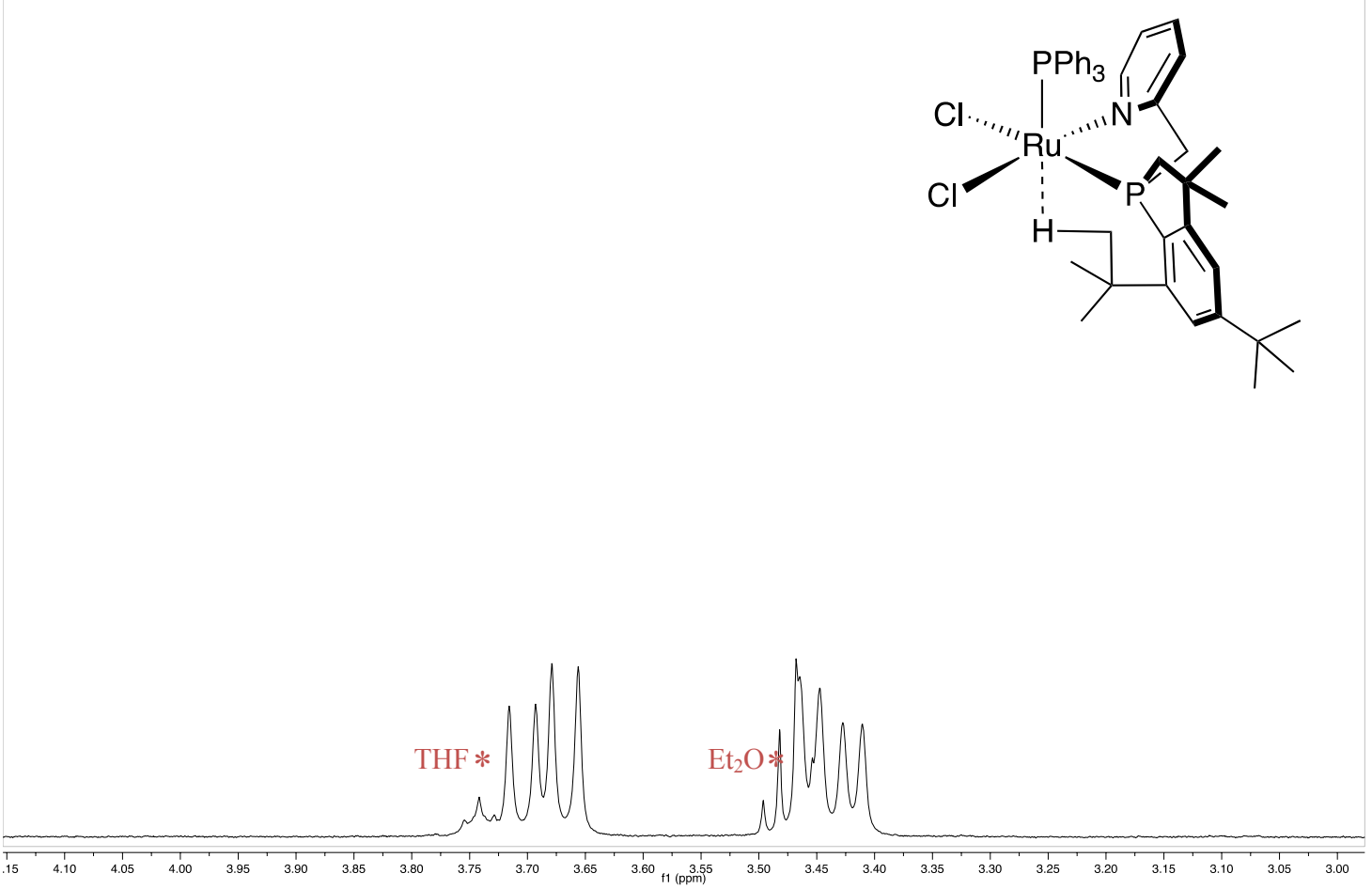
${ }^{1} \mathrm{H}$ NMR (500 MHz, $\mathrm{CDCl}_{3}$ ): Zoomed in on phospholane and $t$-Bu/Me protons

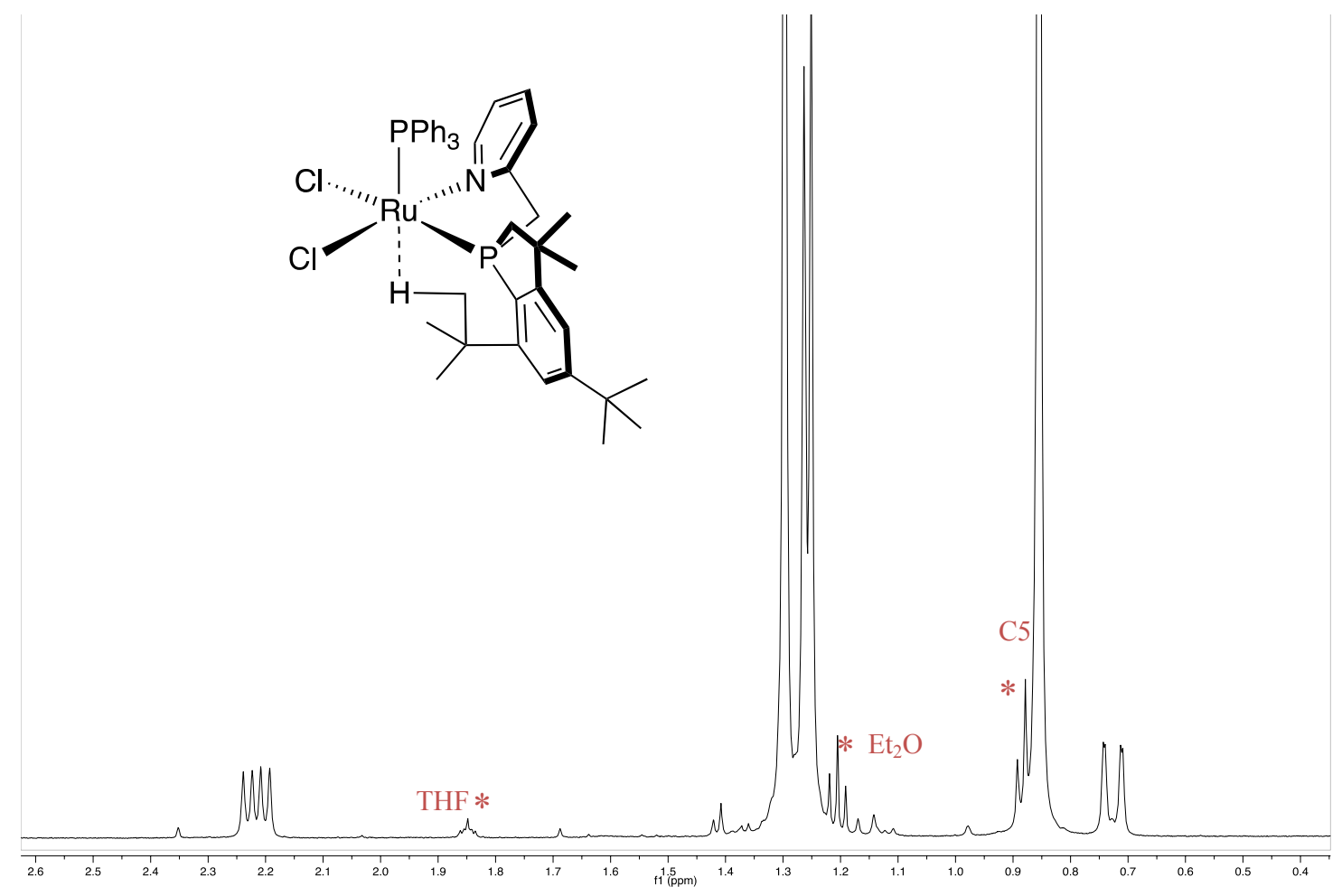

${ }^{13} \mathrm{C}\left\{{ }^{1} \mathrm{H}\right\}$ NMR $\left(125 \mathrm{MHz}, \mathrm{CDCl}_{3}\right)$

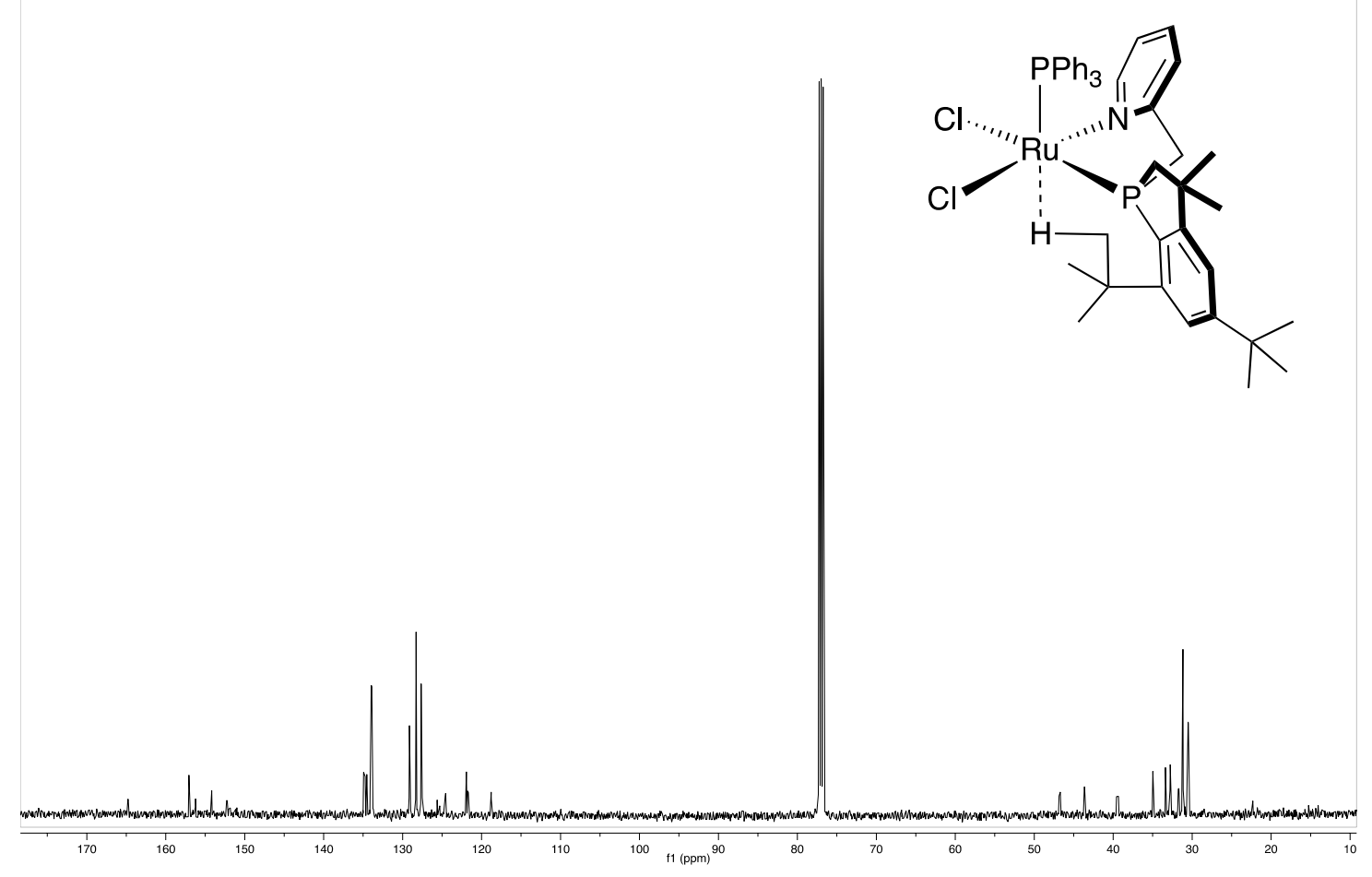


${ }^{13} \mathrm{C}\left\{{ }^{1} \mathrm{H}\right\}$ NMR (125 MHz, $\mathrm{CDCl}_{3}$ ): Aryl Region
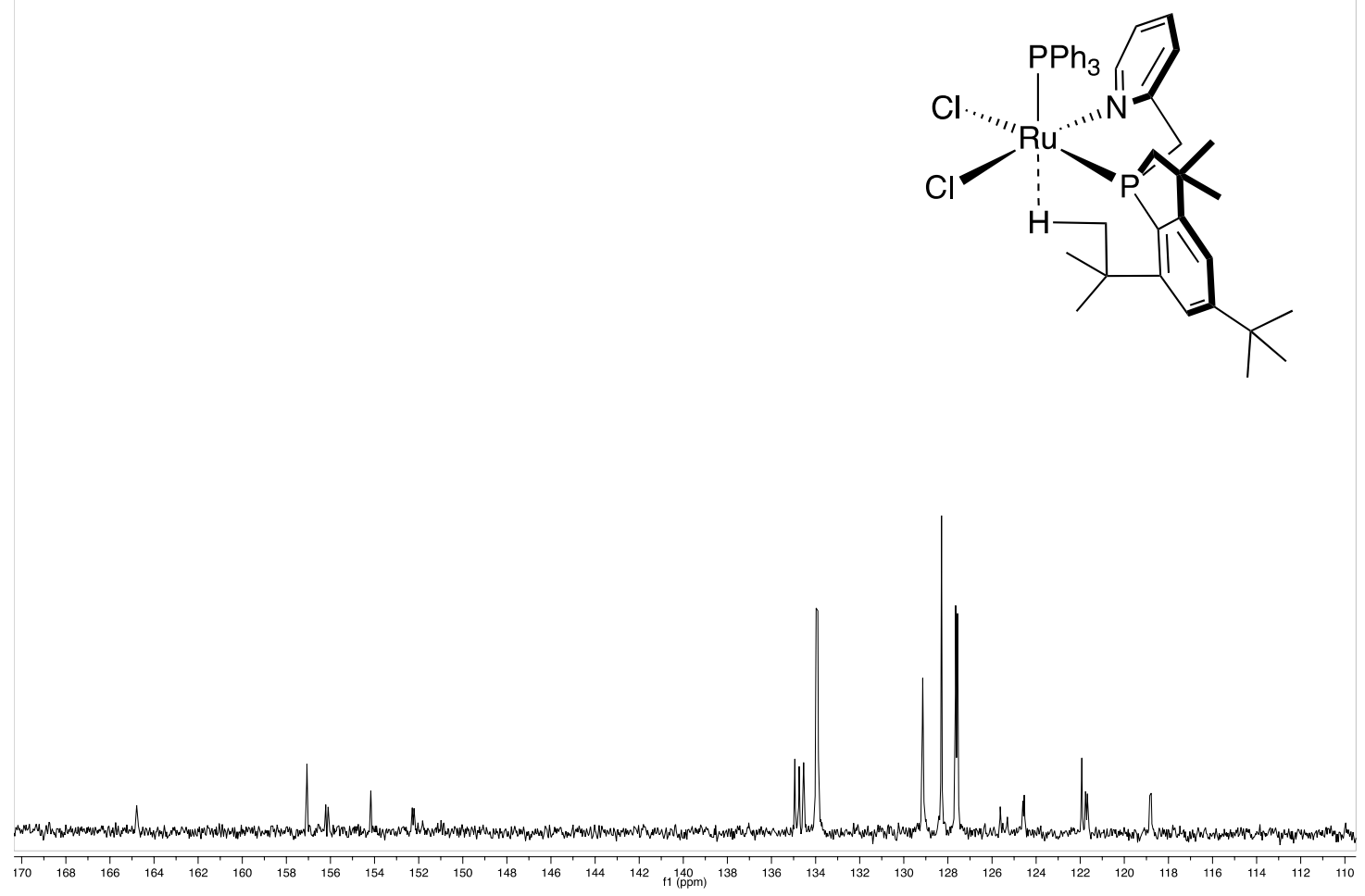

${ }^{13} \mathrm{C}\left\{{ }^{1} \mathrm{H}\right\}$ NMR $\left(125 \mathrm{MHz}, \mathrm{CDCl}_{3}\right)$ : Alkyl Region
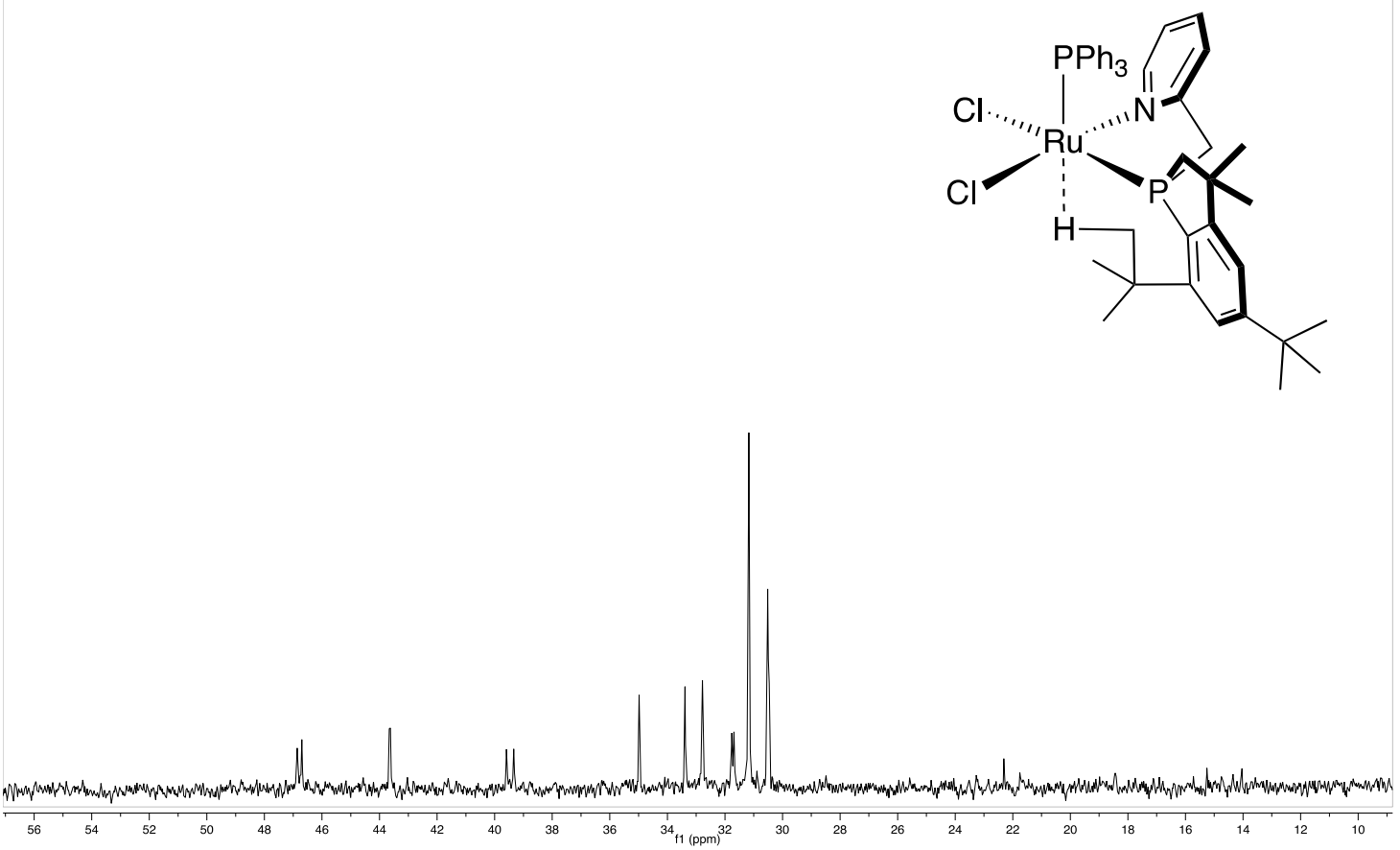


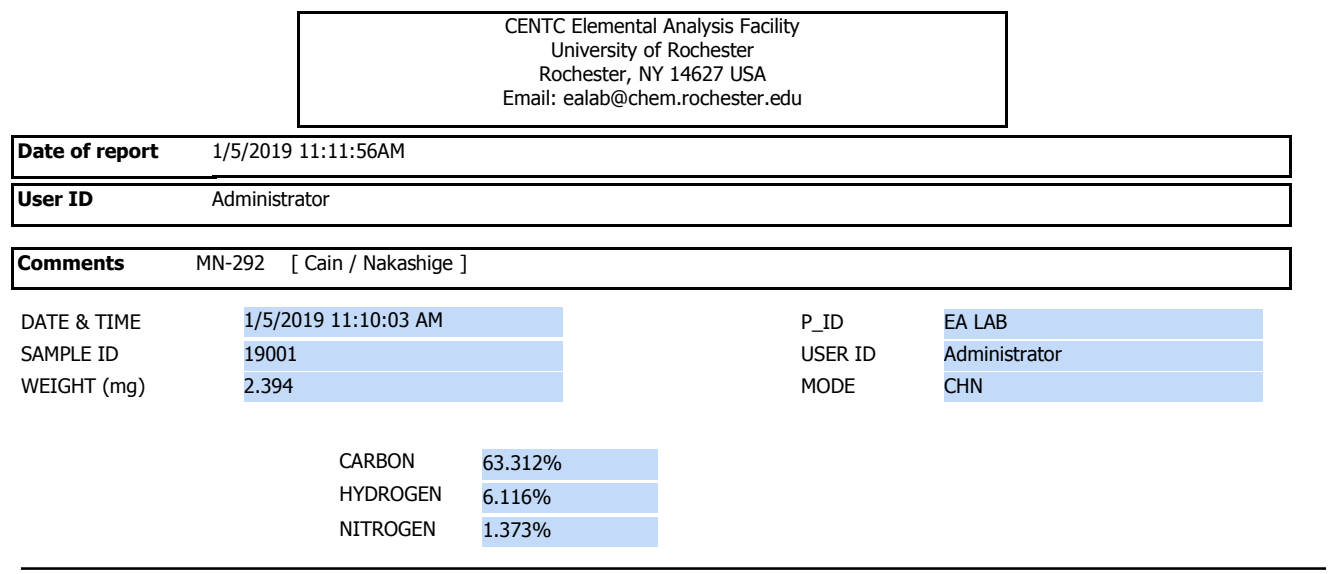

Special Handling

The sample was transferred under argon and was combusted in a tin capsule that was crimp-sealed with a die apparatus.

\section{Acknowledgment}

Analytical data were obtained from the CENTC Elemental Analysis Facility at the University of Rochester, funded by NSF CHE-0650456.

$\underline{\text { Instrumentation }}$

Microanalysis samples were weighed with a PerkinElmer Model AD6000 Autobalance and their compositions were determined with a PerkinElmer 2400 Series II Analyzer. Air-sensitive samples were handled in a VAC

Atmospheres glovebox.

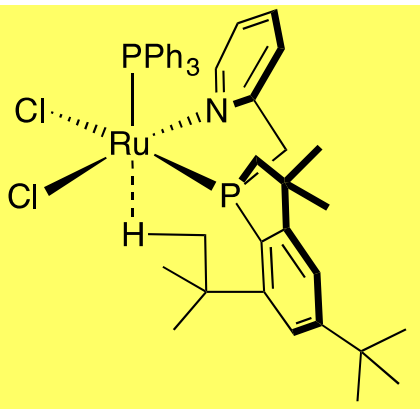

Chemical Formula: $\mathrm{C}_{42} \mathrm{H}_{49} \mathrm{Cl}_{2} \mathrm{NP}_{2} \mathrm{Ru}$

Exact Mass: 801.1761

Molecular Weight: 801.7785

Elemental Analysis: C, 62.92; $\mathrm{H}, 6.16$; $\mathrm{Cl}, 8.84$;

$\mathrm{N}, 1.75 ; \mathrm{P}, 7.73 ; \mathrm{Ru}, 12.61$ 
Synthesis of $6 c\left(\mathrm{R}=\mathrm{CF}_{3}\right)$

$\mathrm{RuCl}_{2}\left(\mathrm{PPh}_{3}\right)_{3}(128 \mathrm{mg}, 0.130 \mathrm{mmol})$ was loaded into a vial, and treated with a solution of $\mathbf{2 c}(60$ $\mathrm{mg}, \quad 0.130 \mathrm{mmol}$ ) in $2 \mathrm{~mL}$ of benzene at room temperature. The dark reaction mixture was transferred to a J-Young tube and monitored by ${ }^{31} \mathrm{P}\left\{{ }^{1} \mathrm{H}\right\}$ NMR spectroscopy. After heating at 60 ${ }^{\circ} \mathrm{C}$ for $240 \mathrm{~h}$, the solution turned dark green, and the ${ }^{31} \mathrm{P}\left\{{ }^{1} \mathrm{H}\right\}$ NMR spectrum showed two doublets at $85.8 \mathrm{ppm}$ and $50.2 \mathrm{ppm}$. The mixture was subsequently filtered through Celite and the filtrate was concentrated under vacuum. The crude residue was recrystallized from THF layered with $\mathrm{Et}_{2} \mathrm{O}$ at $-35^{\circ} \mathrm{C}$ to give green crystals ( $35 \mathrm{mg}, 0.039 \mathrm{mmol}, 30 \%$ yield).

${ }^{31} \mathrm{P}$ NMR $\left(\mathrm{CDCl}_{3}\right): \delta 85.8$ (overlapping dq, $\left.J=42.8,21.5 \mathrm{~Hz}, \mathrm{CH}_{2} \mathrm{P}\right) 50.2\left(\mathrm{~d}, J=42.8 \mathrm{~Hz}, \mathrm{PPh}_{3}\right.$ ). ${ }^{1} \mathrm{H}$ NMR $\left(\mathrm{CDCl}_{3}\right): \delta 7.89$ (t, $\left.J=7.8 \mathrm{~Hz}, 1 \mathrm{H}, \mathrm{Ar}\right), 7.72(\mathrm{~d}, J=7.8 \mathrm{~Hz}, 1 \mathrm{H}, \mathrm{Ar}), 7.68$ (d, $J=7.8$ $\mathrm{Hz}, 1 \mathrm{H}, \mathrm{Ar}), 7.60$ (dd, $J=4.6,2.0 \mathrm{~Hz}, 1 \mathrm{H}, \mathrm{Ar}), 7.40$ (t, $J=7.8 \mathrm{~Hz}, 4 \mathrm{H}, \mathrm{Ar}), 7.30$ (m, 2H, Ar), $7.17(\mathrm{~m}, 7 \mathrm{H}, \mathrm{Ar}), 6.97$ (d, $J=2.0 \mathrm{~Hz}, 1 \mathrm{H}, \mathrm{Ar}), 4.33$ (dd, $\left.J=16.7,10.7 \mathrm{~Hz}, 1 \mathrm{H}, \mathrm{CH}_{2} \mathrm{Ar}\right), 4.18$ (dd, $J=16.7,9.8 \mathrm{~Hz}, 1 \mathrm{H}, \mathrm{CH}_{2} \mathrm{Ar}$ ), 2.27 (dd, $J=16.4,5.1 \mathrm{~Hz}, 1 \mathrm{H}, \mathrm{CH}_{2} \mathrm{P}$ ), 1.99 (dd, $J=16.4$, $\left.10.7 \mathrm{~Hz}, 1 \mathrm{H}, \mathrm{CH}_{2} \mathrm{P}\right), 1.38(\mathrm{~s}, 9 \mathrm{H}, t-\mathrm{Bu}), 1.22(\mathrm{~s}, 9 \mathrm{H}, t-\mathrm{Bu}), 1.10$ (s, $\left.3 \mathrm{H}, \mathrm{Me}\right), 0.79$ (s, 3H, Me). ${ }^{19} \mathrm{~F} \mathrm{NMR}\left(\mathrm{CDCl}_{3}\right): \delta-63.4(\mathrm{~d}, J=21.5 \mathrm{~Hz}) .{ }^{13} \mathrm{C} \mathrm{NMR}\left(125 \mathrm{MHz}, \mathrm{CDCl}_{3}\right): \delta 163.9(\mathrm{Ar}), 157.79$ (Ar), 156.7 (Ar), 153.8 (Ar), 136.9 (Ar), 134.7 (d, $J=10.0 \mathrm{~Hz}, \mathrm{Ar}), 133.5$ (Ar), 133.3 (d, $J=$ $42.8 \mathrm{~Hz}, \mathrm{Ar}), 127.8$ (d, $J=9.6 \mathrm{~Hz}, \mathrm{Ar}), 126.8$ (Ar), 124.7 (Ar), 122.2 (Ar), 118.1 (Ar), 118.0 (Ar), 52.1 (d, $\left.J=21.5 \mathrm{~Hz}, \mathrm{PCH}_{2} \mathrm{Ar}\right), 46.5\left(\mathrm{~d}, J=42.8 \mathrm{~Hz}, \mathrm{PCH}_{2}\right), 43.0\left(\mathrm{CMe}_{2}\right), 38.0\left(\mathrm{CMe}_{3}\right)$, $35.5\left(\mathrm{CMe}_{2}\right), 35.0\left(\mathrm{CMe}_{3}\right), 34.1(t-\mathrm{Bu}), 31.1(t-\mathrm{Bu}), 28.7(\mathrm{~d}, J=10.6 \mathrm{~Hz}, \mathrm{CMe})$. 
${ }^{31} \mathrm{P}\left\{{ }^{1} \mathrm{H}\right\} \mathrm{NMR}\left(\mathrm{CDCl}_{3}\right):$

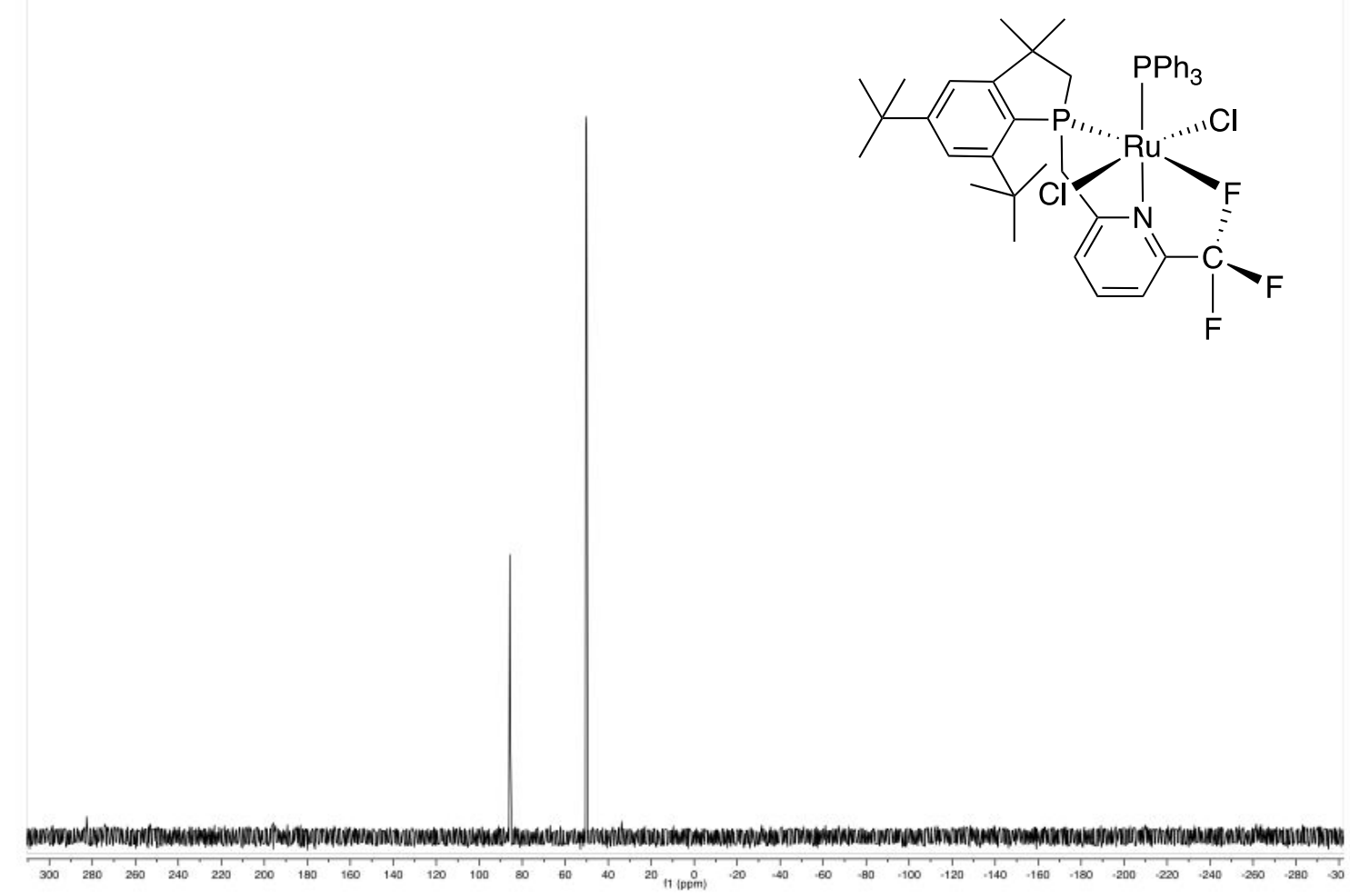

${ }^{31} \mathrm{P}\left\{{ }^{1} \mathrm{H}\right\} \mathrm{NMR}\left(\mathrm{CDCl}_{3}\right):$ Zoom in on phospholane
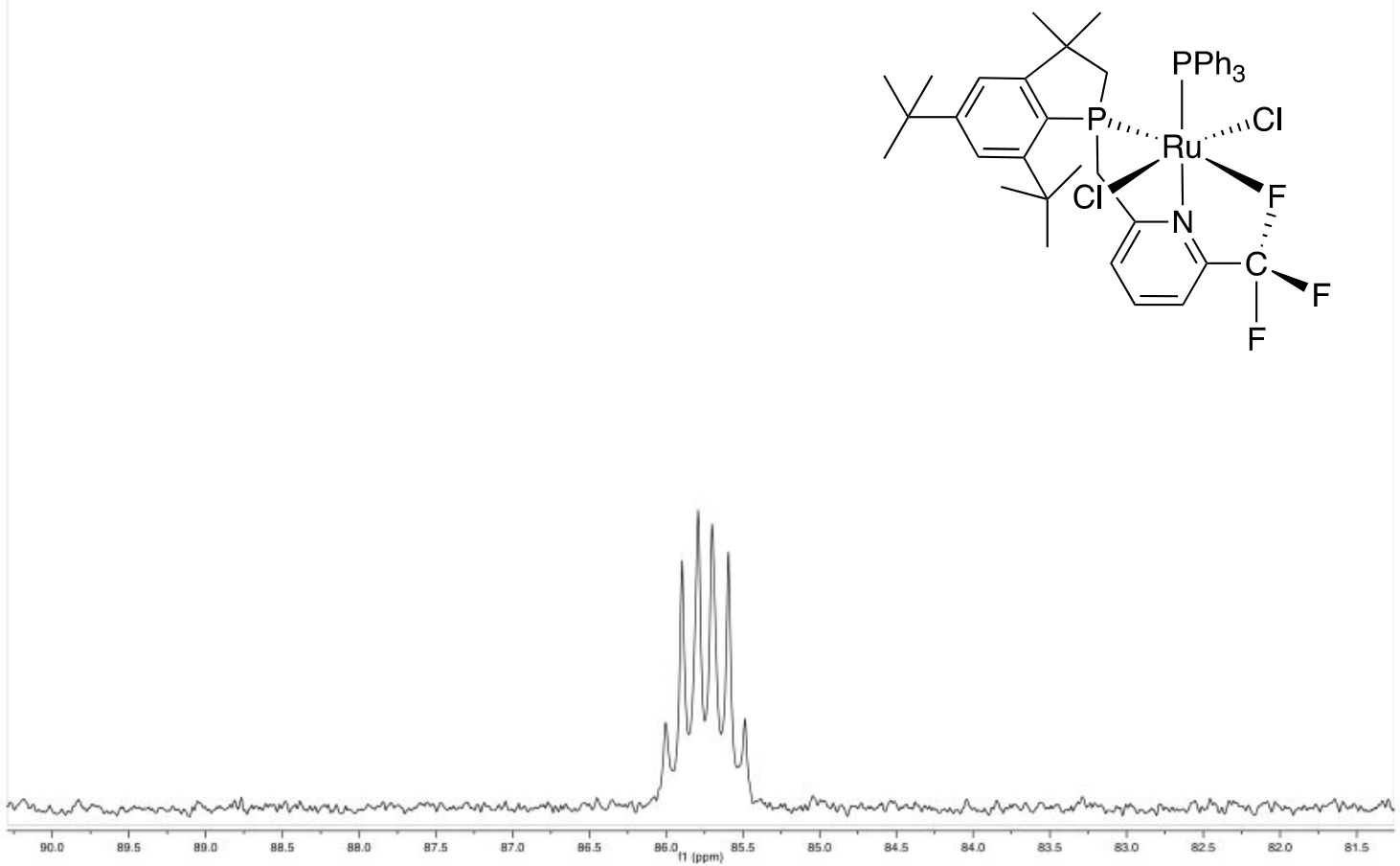
${ }^{31} \mathrm{P}\left\{{ }^{1} \mathrm{H}\right\}$ NMR $\left(\mathrm{CDCl}_{3}\right):$ Zoom in on $\mathrm{PPh}_{3}$

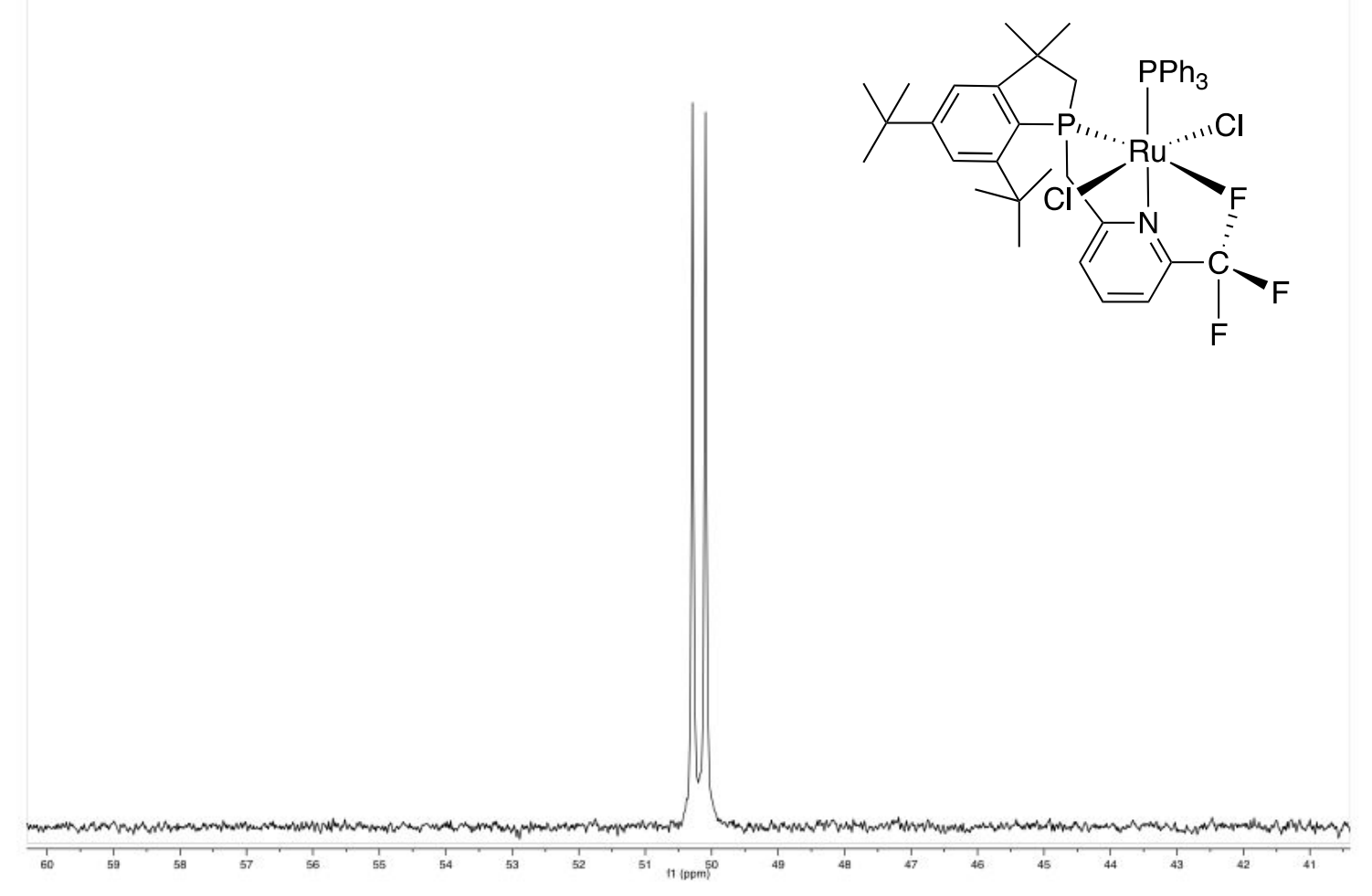

${ }^{1} \mathrm{H} \mathrm{NMR}\left(\mathrm{CDCl}_{3}\right)$
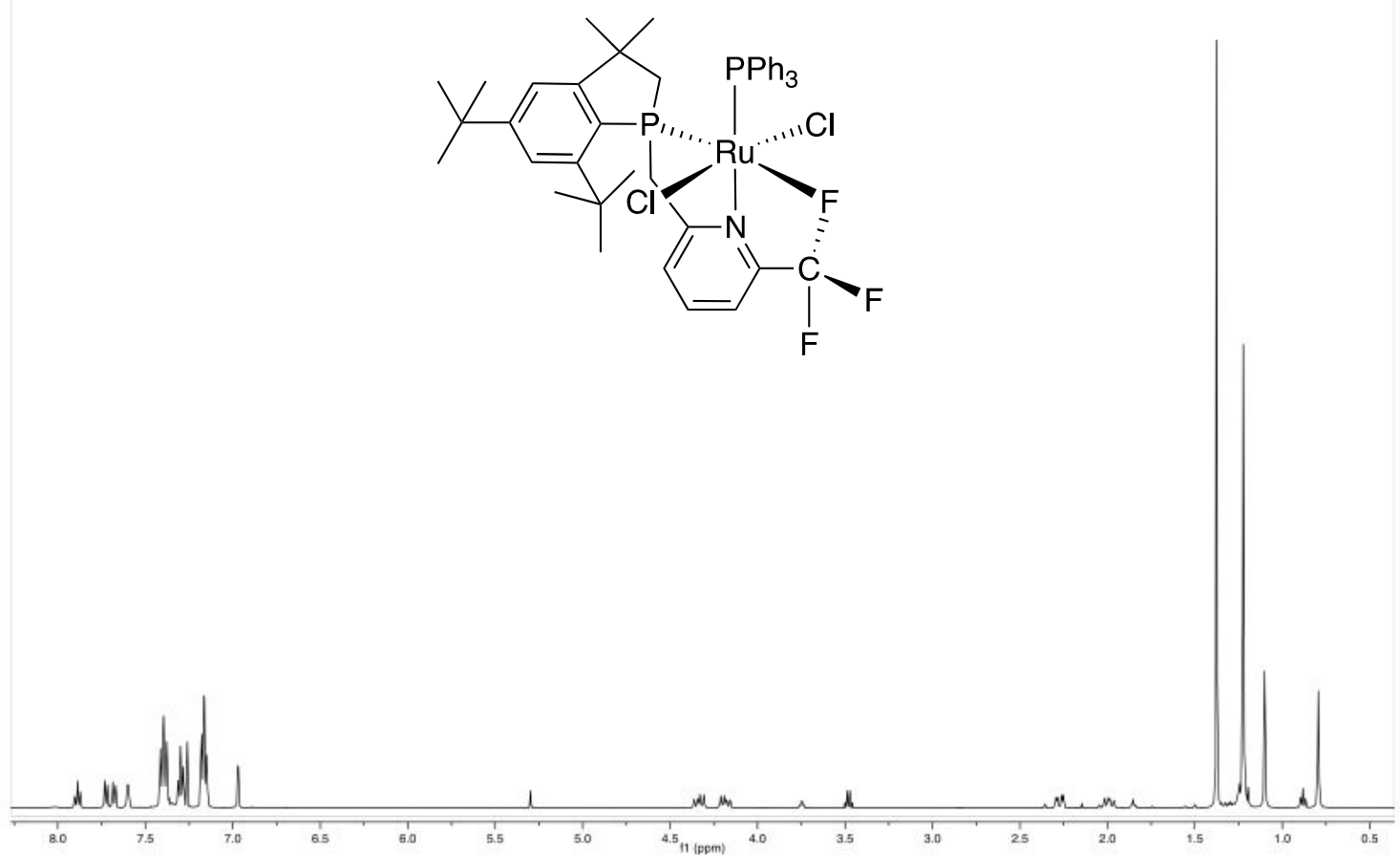
${ }^{1} \mathrm{H}$ NMR $\left(\mathrm{CDCl}_{3}\right)$ : Zoomed in on benzylic protons
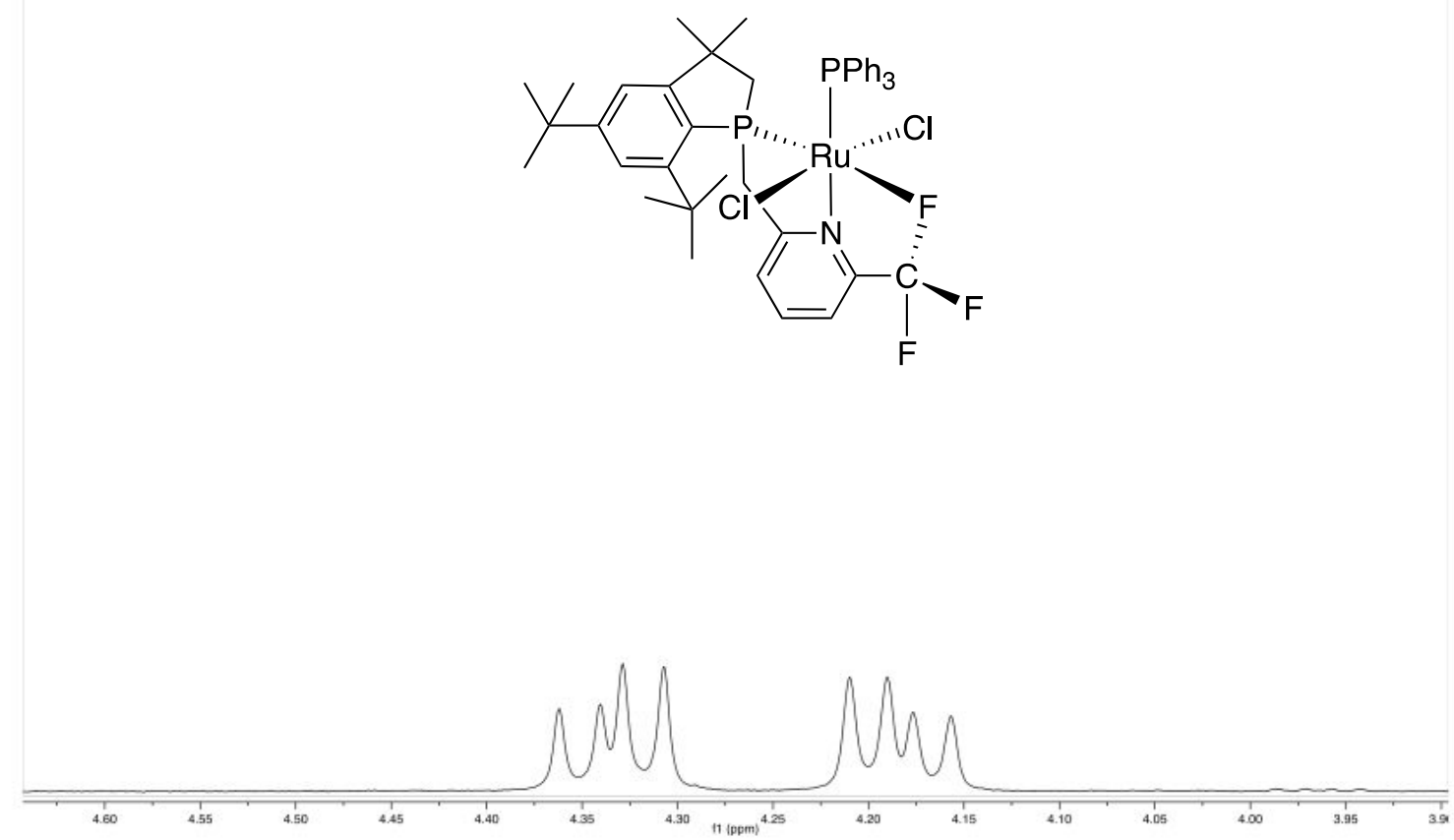

${ }^{1} \mathrm{H} \mathrm{NMR}\left(\mathrm{CDCl}_{3}\right)$ : Zoomed in on aromatic protons
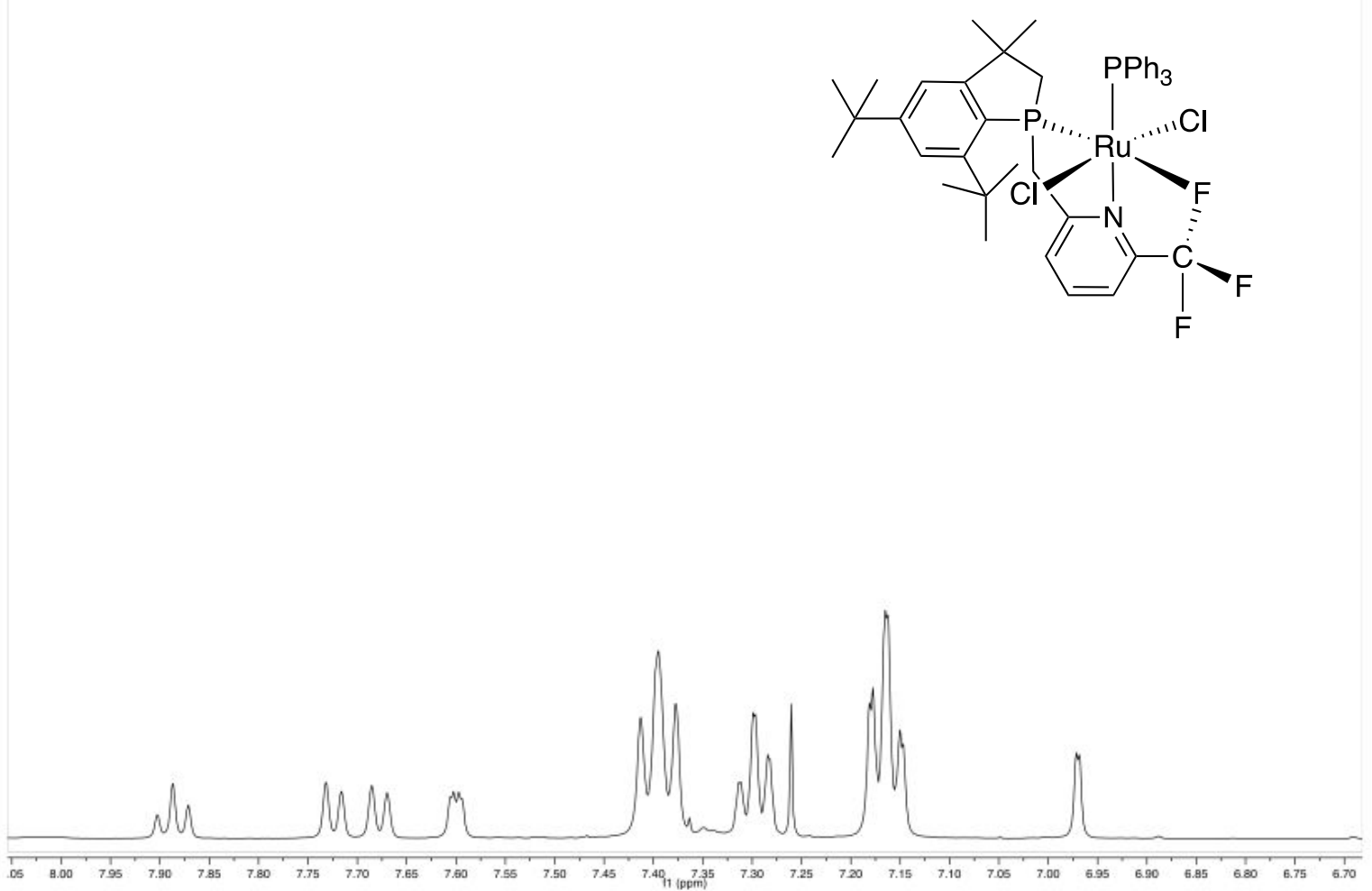
${ }^{19} \mathrm{~F}\left\{{ }^{1} \mathrm{H}\right\} \mathrm{NMR}$

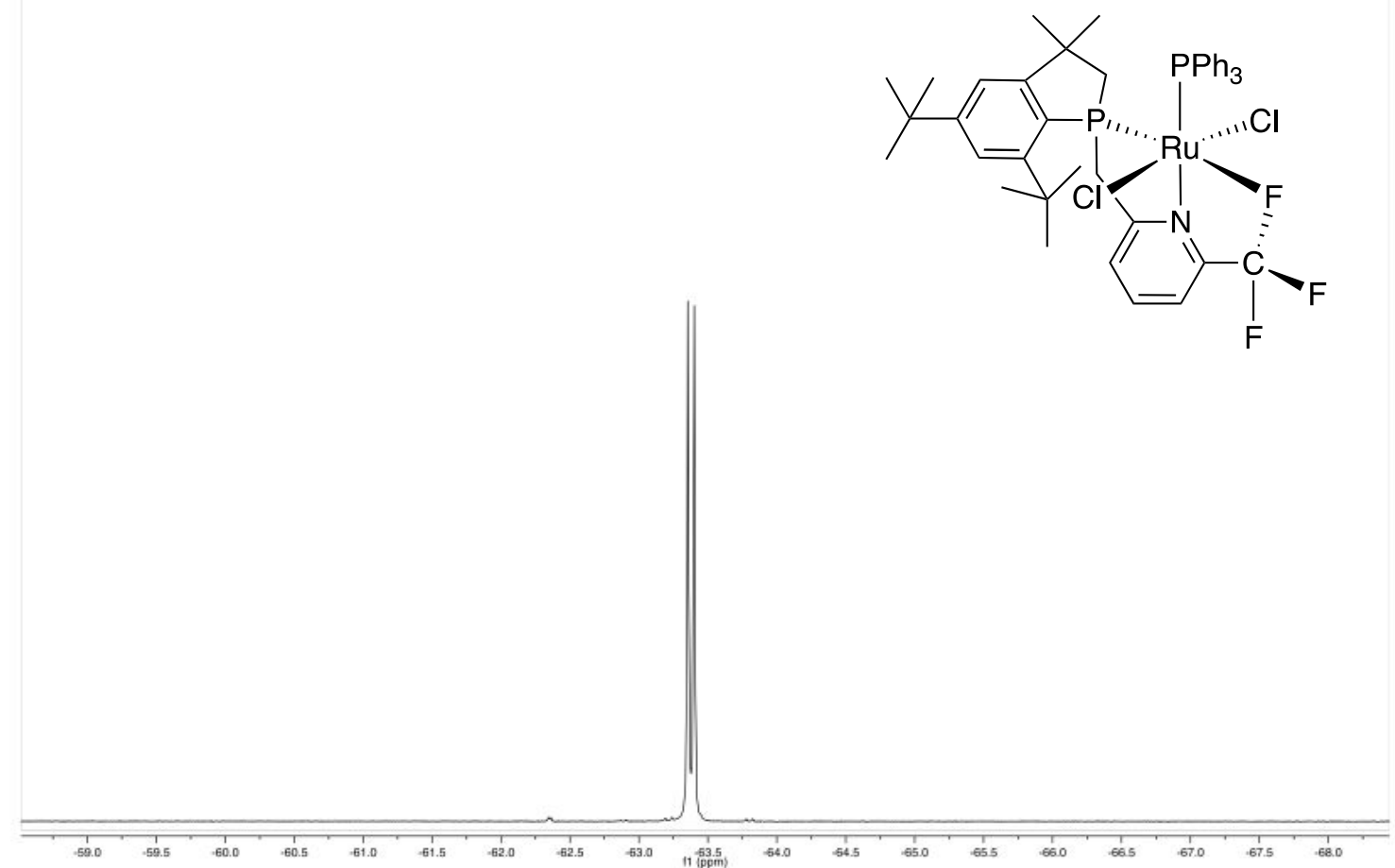

${ }^{13} \mathrm{C}\left\{{ }^{1} \mathrm{H}\right\} \mathrm{NMR}$

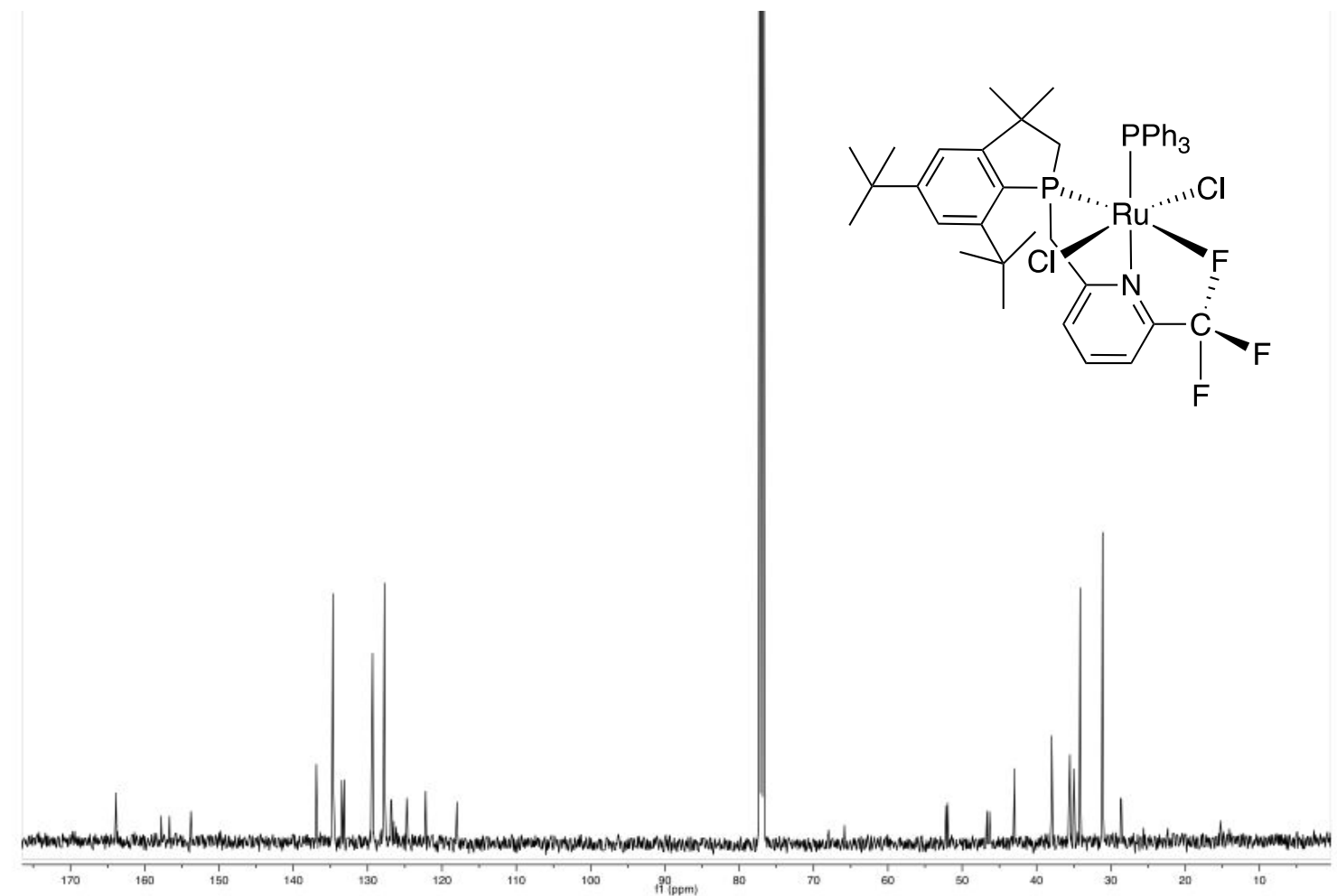


${ }^{13} \mathrm{C}\left\{{ }^{1} \mathrm{H}\right\}$ NMR: Aryl region
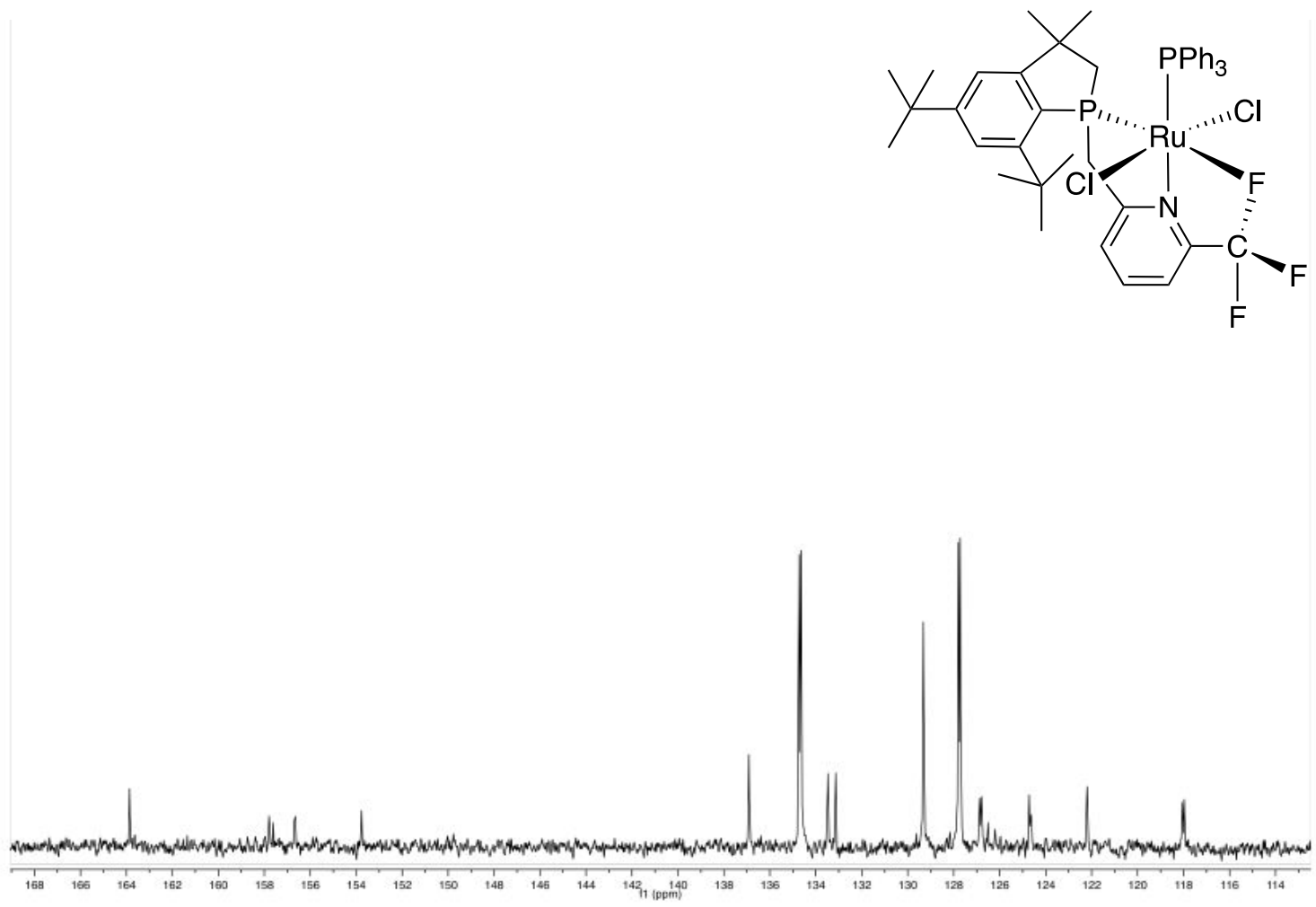

${ }^{13} \mathrm{C}\left\{{ }^{1} \mathrm{H}\right\}$ NMR: Alkyl region
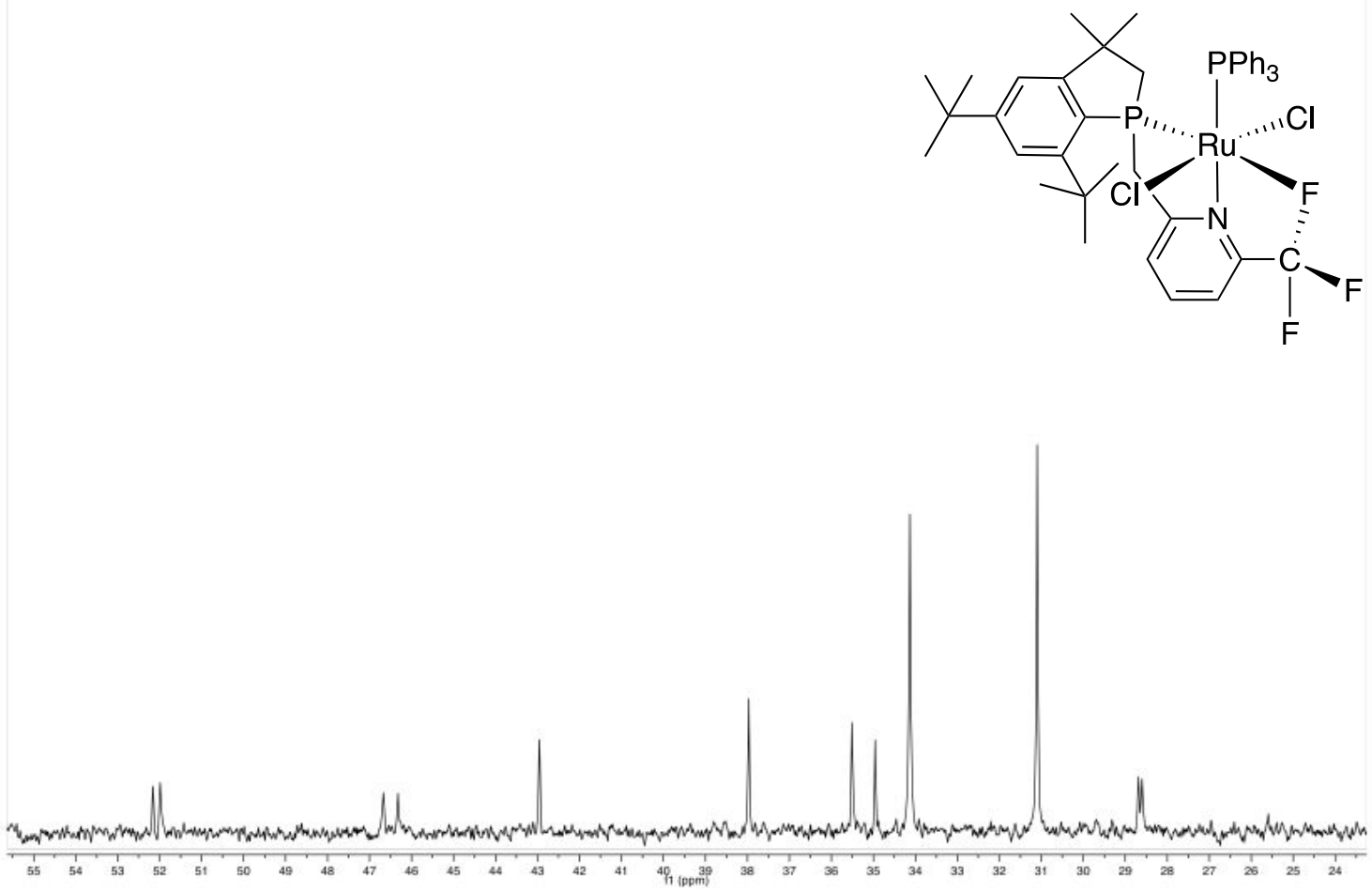


\section{DFT Computation Details}

\section{DFT Calculation Details.}

Full-molecule calculations were carried out using the hybrid B3LYP-D3 functional ${ }^{1}$ with the Grimme dispersion correction $^{2}$ and the LACV3P** basis, set which uses Los Alamos pseudopotentials ${ }^{3}$ for $\mathrm{Ru}$, and the $6-311 \mathrm{G}^{* *}$ basis ${ }^{4}$ for all other atoms, as implemented in the Jaguar $^{5}$ suite of programs. All Natural Bond Orbital calculations ${ }^{6}$ were performed on optimized structures using NBO $6.0,{ }^{7}$ as implemented in Jaguar.

Computed structures were characterized by calculating the vibrational frequencies using second derivative analytic methods and confirming the absence of imaginary frequencies for all minima. Thermodynamic quantities were calculated assuming an ideal gas, and are zero point energy corrected. Graphical representations of structures were made using the CYLView program. ${ }^{8}$

\section{Material Relevant to all DFT output}

Jaguar version 9.6, release 13, Schrodinger, LLC, New York, NY, 2017

basis set: LACV3P**

net molecular charge: 0

multiplicity: 1

Non-default options chosen:

SCF calculation type: DFT(B3LYP-D3)

DFT=B3LYP-D3

Vibrational frequencies and related properties computed from analytic second derivatives Molecular symmetry not used

Energy convergence criterion: $1.00 \mathrm{E}-05$ hartrees

RMS density matrix convergence criterion: $1.00 \mathrm{E}-06$

Highest accuracy cutoffs used in SCF

\section{References.}

(1) (a) Andersson, M. P.; Uvdal, P. J. Phys. Chem. A 2005, 109, 2937-2941; (b) Lee, C.; Yang, W.; Parr, R. G. Phys. Rev. B 1988, 37, 785-789; (c) Stephens, P. J.; Devlin, F. J.; Chabalowski, C. F.; Frisch, M. J. J. Phys. Chem. 1994, 98, 11623-11627; (d) Becke, A. D. J. Chem. Phys. 1993, 98, 1372-1377; (e) Becke, A. D. J. Chem. Phys. 1993, 98, 56485652.

(2) (a) Goerigk, L.; Grimme, S. Phys. Chem. Chem. Phys. 2011, 13, 6670-6688; (b) Grimme, S.; Antony, J.; Ehrlich, S.; Krieg, H. J. Chem. Phys. 2010, 132, 154104.

(3) (a) Dunning, T. H.; Hay, P. J., In Modern Theoretical Chemistry, Vol. 4: Applications of Electronic Structure Theory. Schaefer, H. F., III (Editor); Plenum, NY: 1977 ; (b) Wadt, W. R.; Hay, P. J. J. Chem. Phys. 1985, 82, 284-298; (c) Hay, P. J.; Wadt, W. R. J. Chem. Phys. 1985, 82, 299-310; (d) Hay, P. J.; Wadt, W. R. J. Chem. Phys. 1985, 82, 270-283.

(4) (a) McLean, A. D.; Chandler, G. S. J, Chem. Phys. 1980, 72, 5639-5648; (b) Clark, T.; Chandrasekhar, J.; Spitznagel, G. W.; Schleyer, P. v. R. J. Comput. Chem. 1983, 4, 294- 
301; (c) Krishnan, R.; Binkley, J. S.; Seeger, R.; Pople, J. A. J. Chem. Phys. 1980, 72, 650-654; (d) Frisch, M. J.; Pople, J. A.; Binkley, J. S. J. Chem. Phys. 1984, 80, $3265-$ 3269.

(5) (a) Bochevarov, A. D.; Harder, E.; Hughes, T. F.; Greenwood, J. R.; Braden, D. A.; Philipp, D. M.; Rinaldo, D.; Halls, M. D.; Zhang, J.; Friesner, R. A. Int. J. Quantum Chem. 2013, 113, 2110-2142; (b) Jaguar, versions 7.0-9.6, Schrödinger, LLC, New York, NY: 2007-2018.

(6) (a) Weinhold, F.; Landis, C. R., Discovering Chemistry with Natural Bond Orbitals, John Wiley \& Sons: NJ, 2012; (b) Glendening, E. D.; Landis, C. R.; Weinhold, F. Wiley Interdisciplinary Reviews: Computational Molecular Science 2012, 2, 1-42; (c) Weinhold, F.; Landis, C. R., Valency and Bonding: A Natural Bond Orbital DonorAcceptor Perspective, Cambridge University Press: Cambridge, 2005; (d) Reed, A. E.; Curtiss, L. A.; Weinhold, F. Chem. Rev. 1988, 88, 899-926.

(7) (a) Glendening, E. D.; Landis, C. R.; Weinhold, F. J. Comp. Chem. 2013, 34, 1429-1437; (b) Glendening, E. D.; Badenhoop, J. K.; Reed, A. K.; Carpenter, J. E.; Bohmann, J. A.; Morales, C. M.; Landis, C. R.; Weinhold, F. NBO 6.0, Theoretical Chemistry Institute, University of Wisconsin; Madison: 2013.

(8) Legault, C. Y. CYLview, 1.0b, Université de Sherbrooke: Université de Sherbrooke, 2009. 
3. Experimental Details for Crystal Structure Refinement and Acquisition

All data were collected at $100 \mathrm{~K}$ (except for $2 \mathrm{e}$, which was collected at $200 \mathrm{~K}$ due to an apparent destructive phase change at lower temperatures) on Bruker diffractometers using MoK $\alpha$ radiation. For $\mathbf{4 e}, \mathbf{3 b}$, and $\mathbf{4 b}$, the program SQUEEZE was used to render highly disorder solvent molecules. All specimens of $\mathbf{2 d}$ and $\mathbf{5 a}$ were found to be rotationally twinned. The structure of $\mathbf{2 e}$ contained a disordered $t$-Bu group. 
Table S1. Crystal data and structure refinement for $\mathbf{2 b}$.

Identification code

$\mathrm{MN}-208 \mathrm{a}$

Empirical formula

C25 H36 N P

Formula weight

381.52

Temperature

$100 \mathrm{~K}$

Wavelength

$0.71073 \AA$

Crystal system

Space group

Unit cell dimensions

Orthorhombic

$\mathrm{Pbcn}$

$\begin{array}{ll}\mathrm{a}=18.8900(19) \AA & \alpha=90^{\circ} . \\ \mathrm{b}=11.5502(11) \AA & \beta=90^{\circ} . \\ \mathrm{c}=21.152(3) \AA & \gamma=90^{\circ} .\end{array}$

Volume

Z

Density (calculated)

4614.9(9) $\AA^{3}$

8

Absorption coefficient

$1.098 \mathrm{Mg} / \mathrm{m}^{3}$

$\mathrm{F}(000)$

Crystal size

Theta range for data collection

Index ranges

Reflections collected

$0.128 \mathrm{~mm}^{-1}$

1664

$0.34 \times 0.32 \times 0.1 \mathrm{~mm}^{3}$

2.156 to $26.387^{\circ}$.

$-22<=\mathrm{h}<=23,-10<=\mathrm{k}<=14,-26<=1<=23$

22240

Independent reflections

Completeness to theta $=25.242^{\circ}$

$4730[\mathrm{R}($ int $)=0.0693]$

$99.9 \%$

Absorption correction

Semi-empirical from equivalents

Max. and min. transmission

0.7454 and 0.6699

Refinement method

Data / restraints / parameters

Goodness-of-fit on $\mathrm{F}^{2}$

Full-matrix least-squares on $\mathrm{F}^{2}$

4730 / 0 / 258

1.031

Final $\mathrm{R}$ indices [I $>2 \operatorname{sigma}(\mathrm{I})]$

$\mathrm{R}$ indices (all data)

Extinction coefficient

Largest diff. peak and hole

$\mathrm{R} 1=0.0473, \mathrm{wR} 2=0.0958$

$\mathrm{R} 1=0.0842, \mathrm{wR} 2=0.1093$

$\mathrm{n} / \mathrm{a}$

0.329 and -0.291 e. $\AA^{-3}$ 
Table S2. Atomic coordinates ( $\left.\times 10^{4}\right)$ and equivalent isotropic displacement parameters $\left(\AA^{2} \times 10^{3}\right)$ for $\mathbf{2 b}$. $U(\mathrm{eq})$ is defined as one third of the trace of the orthogonalized $\mathrm{U}^{\mathrm{ij}}$ tensor.

\begin{tabular}{|c|c|c|c|c|}
\hline & $\mathrm{x}$ & $\mathrm{y}$ & z & $\mathrm{U}(\mathrm{eq})$ \\
\hline $\mathrm{P}(1)$ & $3155(1)$ & $4257(1)$ & $6319(1)$ & $17(1)$ \\
\hline $\mathrm{N}(1)$ & $4644(1)$ & $3366(2)$ & $6621(1)$ & $19(1)$ \\
\hline $\mathrm{C}(11)$ & $920(1)$ & $2955(2)$ & $5728(1)$ & $13(1)$ \\
\hline$C(10)$ & $1112(1)$ & $3002(2)$ & $6358(1)$ & $14(1)$ \\
\hline$C(12)$ & 1352(1) & $3539(2)$ & $5300(1)$ & $14(1)$ \\
\hline$C(13)$ & 1993(1) & $4050(2)$ & $5471(1)$ & $13(1)$ \\
\hline$C(6)$ & $4170(1)$ & $2528(2)$ & $6480(1)$ & $18(1)$ \\
\hline$C(7)$ & $3439(1)$ & $2885(2)$ & $6351(1)$ & $16(1)$ \\
\hline$C(9)$ & 1733(1) & $3532(2)$ & $6573(1)$ & $13(1)$ \\
\hline $\mathrm{C}(8)$ & $2213(1)$ & $3965(2)$ & $6114(1)$ & $12(1)$ \\
\hline$C(18)$ & $245(1)$ & 2351(2) & $5498(1)$ & $16(1)$ \\
\hline$C(14)$ & $1828(1)$ & $3648(2)$ & $7300(1)$ & $15(1)$ \\
\hline$C(24)$ & $2584(1)$ & $5942(2)$ & $5177(1)$ & $20(1)$ \\
\hline$C(22)$ & $2415(1)$ & $4706(2)$ & $4954(1)$ & $14(1)$ \\
\hline$C(2)$ & 5311(1) & $3052(2)$ & $6742(1)$ & $22(1)$ \\
\hline$C(5)$ & $4356(1)$ & $1358(2)$ & $6453(1)$ & $22(1)$ \\
\hline$C(25)$ & 3099(1) & $4073(2)$ & $4760(1)$ & $20(1)$ \\
\hline$C(17)$ & $2188(1)$ & 4794(2) & $7474(1)$ & $24(1)$ \\
\hline$C(23)$ & 1984(1) & $4848(2)$ & $4342(1)$ & $22(1)$ \\
\hline$C(4)$ & $5048(1)$ & $1049(2)$ & $6584(1)$ & $28(1)$ \\
\hline$C(15)$ & $1100(1)$ & $3676(2)$ & $7632(1)$ & $24(1)$ \\
\hline $\mathrm{C}(21)$ & $412(1)$ & $1563(2)$ & $4936(1)$ & $25(1)$ \\
\hline$C(19)$ & $-88(1)$ & $1598(2)$ & $6015(1)$ & $29(1)$ \\
\hline$C(20)$ & $-288(1)$ & $3275(2)$ & $5291(1)$ & $31(1)$ \\
\hline$C(3)$ & $5528(1)$ & $1896(2)$ & $6735(1)$ & $27(1)$ \\
\hline$C(16)$ & $2239(1)$ & $2627(2)$ & $7580(1)$ & $23(1)$ \\
\hline$C(1)$ & 5823(1) & 4014(2) & 6889(1) & $34(1)$ \\
\hline
\end{tabular}


Table S3. Bond lengths $[\AA]$ and angles $\left[{ }^{\circ}\right]$ for $\mathbf{2 b}$.

\begin{tabular}{|c|c|}
\hline $\mathrm{P}(1)-\mathrm{C}(7)$ & $1.674(2)$ \\
\hline $\mathrm{P}(1)-\mathrm{C}(8)$ & $1.8607(19)$ \\
\hline $\mathrm{N}(1)-\mathrm{C}(6)$ & $1.351(3)$ \\
\hline $\mathrm{N}(1)-\mathrm{C}(2)$ & $1.337(2)$ \\
\hline$C(11)-C(10)$ & $1.383(3)$ \\
\hline$C(11)-C(12)$ & $1.392(3)$ \\
\hline $\mathrm{C}(11)-\mathrm{C}(18)$ & $1.532(2)$ \\
\hline $\mathrm{C}(10)-\mathrm{H}(10)$ & 0.9500 \\
\hline$C(10)-C(9)$ & $1.399(3)$ \\
\hline $\mathrm{C}(12)-\mathrm{H}(12)$ & 0.9500 \\
\hline$C(12)-C(13)$ & $1.394(3)$ \\
\hline$C(13)-C(8)$ & $1.425(3)$ \\
\hline$C(13)-C(22)$ & $1.552(3)$ \\
\hline$C(6)-C(7)$ & $1.466(3)$ \\
\hline$C(6)-C(5)$ & $1.398(3)$ \\
\hline $\mathrm{C}(7)-\mathrm{H}(7)$ & $0.95(2)$ \\
\hline $\mathrm{C}(9)-\mathrm{C}(8)$ & $1.421(3)$ \\
\hline C(9)-C(14) & $1.553(3)$ \\
\hline $\mathrm{C}(18)-\mathrm{C}(21)$ & $1.530(3)$ \\
\hline $\mathrm{C}(18)-\mathrm{C}(19)$ & $1.532(3)$ \\
\hline$C(18)-C(20)$ & $1.531(3)$ \\
\hline$C(14)-C(17)$ & $1.533(3)$ \\
\hline$C(14)-C(15)$ & $1.544(3)$ \\
\hline$C(14)-C(16)$ & $1.532(3)$ \\
\hline $\mathrm{C}(24)-\mathrm{H}(24 \mathrm{~A})$ & 0.9800 \\
\hline $\mathrm{C}(24)-\mathrm{H}(24 \mathrm{~B})$ & 0.9800 \\
\hline $\mathrm{C}(24)-\mathrm{H}(24 \mathrm{C})$ & 0.9800 \\
\hline$C(24)-C(22)$ & $1.537(3)$ \\
\hline$C(22)-C(25)$ & $1.540(3)$ \\
\hline$C(22)-C(23)$ & $1.539(3)$ \\
\hline$C(2)-C(3)$ & $1.397(3)$ \\
\hline $\mathrm{C}(2)-\mathrm{C}(1)$ & $1.504(3)$ \\
\hline $\mathrm{C}(5)-\mathrm{H}(5)$ & 0.9500 \\
\hline$C(5)-C(4)$ & $1.383(3)$ \\
\hline
\end{tabular}




\begin{tabular}{|c|c|}
\hline $\mathrm{C}(25)-\mathrm{H}(25 \mathrm{~A})$ & 0.9800 \\
\hline $\mathrm{C}(25)-\mathrm{H}(25 \mathrm{~B})$ & 0.9800 \\
\hline $\mathrm{C}(25)-\mathrm{H}(25 \mathrm{C})$ & 0.9800 \\
\hline $\mathrm{C}(17)-\mathrm{H}(17 \mathrm{~A})$ & 0.9800 \\
\hline $\mathrm{C}(17)-\mathrm{H}(17 \mathrm{~B})$ & 0.9800 \\
\hline $\mathrm{C}(17)-\mathrm{H}(17 \mathrm{C})$ & 0.9800 \\
\hline $\mathrm{C}(23)-\mathrm{H}(23 \mathrm{~A})$ & 0.9800 \\
\hline $\mathrm{C}(23)-\mathrm{H}(23 \mathrm{~B})$ & 0.9800 \\
\hline $\mathrm{C}(23)-\mathrm{H}(23 \mathrm{C})$ & 0.9800 \\
\hline $\mathrm{C}(4)-\mathrm{H}(4)$ & 0.9500 \\
\hline$C(4)-C(3)$ & $1.372(3)$ \\
\hline $\mathrm{C}(15)-\mathrm{H}(15 \mathrm{~A})$ & 0.9800 \\
\hline $\mathrm{C}(15)-\mathrm{H}(15 \mathrm{~B})$ & 0.9800 \\
\hline $\mathrm{C}(15)-\mathrm{H}(15 \mathrm{C})$ & 0.9800 \\
\hline $\mathrm{C}(21)-\mathrm{H}(21 \mathrm{~A})$ & 0.9800 \\
\hline $\mathrm{C}(21)-\mathrm{H}(21 \mathrm{~B})$ & 0.9800 \\
\hline $\mathrm{C}(21)-\mathrm{H}(21 \mathrm{C})$ & 0.9800 \\
\hline C(19)-H(19A) & 0.9800 \\
\hline C(19)-H(19B) & 0.9800 \\
\hline C(19)-H(19C) & 0.9800 \\
\hline $\mathrm{C}(20)-\mathrm{H}(20 \mathrm{~A})$ & 0.9800 \\
\hline C(20)-H(20B) & 0.9800 \\
\hline $\mathrm{C}(20)-\mathrm{H}(20 \mathrm{C})$ & 0.9800 \\
\hline $\mathrm{C}(3)-\mathrm{H}(3)$ & 0.9500 \\
\hline $\mathrm{C}(16)-\mathrm{H}(16 \mathrm{~A})$ & 0.9800 \\
\hline $\mathrm{C}(16)-\mathrm{H}(16 \mathrm{~B})$ & 0.9800 \\
\hline C(16)-H(16C) & 0.9800 \\
\hline $\mathrm{C}(1)-\mathrm{H}(1 \mathrm{~A})$ & 0.9800 \\
\hline $\mathrm{C}(1)-\mathrm{H}(1 \mathrm{~B})$ & 0.9800 \\
\hline $\mathrm{C}(1)-\mathrm{H}(1 \mathrm{C})$ & 0.9800 \\
\hline $\mathrm{C}(7)-\mathrm{P}(1)-\mathrm{C}(8)$ & $98.35(9)$ \\
\hline $\mathrm{C}(2)-\mathrm{N}(1)-\mathrm{C}(6)$ & $118.26(18)$ \\
\hline$C(10)-C(11)-C(12)$ & $117.02(17)$ \\
\hline$C(10)-C(11)-C(18)$ & $122.76(17)$ \\
\hline$C(12)-C(11)-C(18)$ & $120.14(17)$ \\
\hline
\end{tabular}




\begin{tabular}{|c|c|}
\hline $\mathrm{C}(11)-\mathrm{C}(10)-\mathrm{H}(10)$ & 118.3 \\
\hline $\mathrm{C}(11)-\mathrm{C}(10)-\mathrm{C}(9)$ & $123.37(17)$ \\
\hline $\mathrm{C}(9)-\mathrm{C}(10)-\mathrm{H}(10)$ & 118.3 \\
\hline $\mathrm{C}(11)-\mathrm{C}(12)-\mathrm{H}(12)$ & 118.5 \\
\hline $\mathrm{C}(11)-\mathrm{C}(12)-\mathrm{C}(13)$ & $123.07(17)$ \\
\hline $\mathrm{C}(13)-\mathrm{C}(12)-\mathrm{H}(12)$ & 118.5 \\
\hline $\mathrm{C}(12)-\mathrm{C}(13)-\mathrm{C}(8)$ & $118.14(17)$ \\
\hline $\mathrm{C}(12)-\mathrm{C}(13)-\mathrm{C}(22)$ & $118.11(16)$ \\
\hline $\mathrm{C}(8)-\mathrm{C}(13)-\mathrm{C}(22)$ & $123.74(16)$ \\
\hline N(1)-C(6)-C(7) & $117.62(18)$ \\
\hline $\mathrm{N}(1)-\mathrm{C}(6)-\mathrm{C}(5)$ & $122.33(18)$ \\
\hline$C(5)-C(6)-C(7)$ & $120.05(19)$ \\
\hline $\mathrm{P}(1)-\mathrm{C}(7)-\mathrm{H}(7)$ & $120.3(12)$ \\
\hline $\mathrm{C}(6)-\mathrm{C}(7)-\mathrm{P}(1)$ & $125.15(16)$ \\
\hline $\mathrm{C}(6)-\mathrm{C}(7)-\mathrm{H}(7)$ & $114.5(12)$ \\
\hline$C(10)-C(9)-C(8)$ & $117.85(17)$ \\
\hline$C(10)-C(9)-C(14)$ & $117.16(16)$ \\
\hline$C(8)-C(9)-C(14)$ & $124.93(16)$ \\
\hline $\mathrm{C}(13)-\mathrm{C}(8)-\mathrm{P}(1)$ & $119.31(14)$ \\
\hline $\mathrm{C}(9)-\mathrm{C}(8)-\mathrm{P}(1)$ & $120.96(14)$ \\
\hline $\mathrm{C}(9)-\mathrm{C}(8)-\mathrm{C}(13)$ & $119.33(17)$ \\
\hline $\mathrm{C}(21)-\mathrm{C}(18)-\mathrm{C}(11)$ & $110.23(15)$ \\
\hline $\mathrm{C}(21)-\mathrm{C}(18)-\mathrm{C}(19)$ & $107.49(17)$ \\
\hline $\mathrm{C}(21)-\mathrm{C}(18)-\mathrm{C}(20)$ & $109.13(17)$ \\
\hline $\mathrm{C}(19)-\mathrm{C}(18)-\mathrm{C}(11)$ & $112.01(16)$ \\
\hline$C(20)-C(18)-C(11)$ & $108.70(16)$ \\
\hline $\mathrm{C}(20)-\mathrm{C}(18)-\mathrm{C}(19)$ & $109.23(17)$ \\
\hline $\mathrm{C}(17)-\mathrm{C}(14)-\mathrm{C}(9)$ & $111.33(16)$ \\
\hline $\mathrm{C}(17)-\mathrm{C}(14)-\mathrm{C}(15)$ & $105.58(16)$ \\
\hline$C(15)-C(14)-C(9)$ & $110.40(15)$ \\
\hline$C(16)-C(14)-C(9)$ & $112.04(15)$ \\
\hline $\mathrm{C}(16)-\mathrm{C}(14)-\mathrm{C}(17)$ & $110.28(16)$ \\
\hline$C(16)-C(14)-C(15)$ & $106.92(16)$ \\
\hline $\mathrm{H}(24 \mathrm{~A})-\mathrm{C}(24)-\mathrm{H}(24 \mathrm{~B})$ & 109.5 \\
\hline $\mathrm{H}(24 \mathrm{~A})-\mathrm{C}(24)-\mathrm{H}(24 \mathrm{C})$ & 109.5 \\
\hline $\mathrm{H}(24 \mathrm{~B})-\mathrm{C}(24)-\mathrm{H}(24 \mathrm{C})$ & 109.5 \\
\hline
\end{tabular}




\begin{tabular}{|c|c|}
\hline $\mathrm{C}(22)-\mathrm{C}(24)-\mathrm{H}(24 \mathrm{~A})$ & 109.5 \\
\hline $\mathrm{C}(22)-\mathrm{C}(24)-\mathrm{H}(24 \mathrm{~B})$ & 109.5 \\
\hline $\mathrm{C}(22)-\mathrm{C}(24)-\mathrm{H}(24 \mathrm{C})$ & 109.5 \\
\hline$C(24)-C(22)-C(13)$ & $110.09(15)$ \\
\hline $\mathrm{C}(24)-\mathrm{C}(22)-\mathrm{C}(25)$ & $110.41(15)$ \\
\hline$C(24)-C(22)-C(23)$ & $105.64(16)$ \\
\hline$C(25)-C(22)-C(13)$ & $112.81(15)$ \\
\hline$C(23)-C(22)-C(13)$ & $111.87(15)$ \\
\hline$C(23)-C(22)-C(25)$ & $105.71(15)$ \\
\hline $\mathrm{N}(1)-\mathrm{C}(2)-\mathrm{C}(3)$ & $122.2(2)$ \\
\hline $\mathrm{N}(1)-\mathrm{C}(2)-\mathrm{C}(1)$ & $116.4(2)$ \\
\hline $\mathrm{C}(3)-\mathrm{C}(2)-\mathrm{C}(1)$ & $121.33(19)$ \\
\hline $\mathrm{C}(6)-\mathrm{C}(5)-\mathrm{H}(5)$ & 120.7 \\
\hline$C(4)-C(5)-C(6)$ & $118.6(2)$ \\
\hline $\mathrm{C}(4)-\mathrm{C}(5)-\mathrm{H}(5)$ & 120.7 \\
\hline $\mathrm{C}(22)-\mathrm{C}(25)-\mathrm{H}(25 \mathrm{~A})$ & 109.5 \\
\hline $\mathrm{C}(22)-\mathrm{C}(25)-\mathrm{H}(25 \mathrm{~B})$ & 109.5 \\
\hline $\mathrm{C}(22)-\mathrm{C}(25)-\mathrm{H}(25 \mathrm{C})$ & 109.5 \\
\hline $\mathrm{H}(25 \mathrm{~A})-\mathrm{C}(25)-\mathrm{H}(25 \mathrm{~B})$ & 109.5 \\
\hline $\mathrm{H}(25 \mathrm{~A})-\mathrm{C}(25)-\mathrm{H}(25 \mathrm{C})$ & 109.5 \\
\hline $\mathrm{H}(25 \mathrm{~B})-\mathrm{C}(25)-\mathrm{H}(25 \mathrm{C})$ & 109.5 \\
\hline $\mathrm{C}(14)-\mathrm{C}(17)-\mathrm{H}(17 \mathrm{~A})$ & 109.5 \\
\hline $\mathrm{C}(14)-\mathrm{C}(17)-\mathrm{H}(17 \mathrm{~B})$ & 109.5 \\
\hline $\mathrm{C}(14)-\mathrm{C}(17)-\mathrm{H}(17 \mathrm{C})$ & 109.5 \\
\hline $\mathrm{H}(17 \mathrm{~A})-\mathrm{C}(17)-\mathrm{H}(17 \mathrm{~B})$ & 109.5 \\
\hline $\mathrm{H}(17 \mathrm{~A})-\mathrm{C}(17)-\mathrm{H}(17 \mathrm{C})$ & 109.5 \\
\hline $\mathrm{H}(17 \mathrm{~B})-\mathrm{C}(17)-\mathrm{H}(17 \mathrm{C})$ & 109.5 \\
\hline $\mathrm{C}(22)-\mathrm{C}(23)-\mathrm{H}(23 \mathrm{~A})$ & 109.5 \\
\hline $\mathrm{C}(22)-\mathrm{C}(23)-\mathrm{H}(23 \mathrm{~B})$ & 109.5 \\
\hline $\mathrm{C}(22)-\mathrm{C}(23)-\mathrm{H}(23 \mathrm{C})$ & 109.5 \\
\hline $\mathrm{H}(23 \mathrm{~A})-\mathrm{C}(23)-\mathrm{H}(23 \mathrm{~B})$ & 109.5 \\
\hline $\mathrm{H}(23 \mathrm{~A})-\mathrm{C}(23)-\mathrm{H}(23 \mathrm{C})$ & 109.5 \\
\hline $\mathrm{H}(23 \mathrm{~B})-\mathrm{C}(23)-\mathrm{H}(23 \mathrm{C})$ & 109.5 \\
\hline $\mathrm{C}(5)-\mathrm{C}(4)-\mathrm{H}(4)$ & 120.4 \\
\hline$C(3)-C(4)-C(5)$ & $119.2(2)$ \\
\hline $\mathrm{C}(3)-\mathrm{C}(4)-\mathrm{H}(4)$ & 120.4 \\
\hline
\end{tabular}




\begin{tabular}{|c|c|}
\hline $\mathrm{C}(14)-\mathrm{C}(15)-\mathrm{H}(15 \mathrm{~A})$ & 109.5 \\
\hline $\mathrm{C}(14)-\mathrm{C}(15)-\mathrm{H}(15 \mathrm{~B})$ & 109.5 \\
\hline $\mathrm{C}(14)-\mathrm{C}(15)-\mathrm{H}(15 \mathrm{C})$ & 109.5 \\
\hline $\mathrm{H}(15 \mathrm{~A})-\mathrm{C}(15)-\mathrm{H}(15 \mathrm{~B})$ & 109.5 \\
\hline $\mathrm{H}(15 \mathrm{~A})-\mathrm{C}(15)-\mathrm{H}(15 \mathrm{C})$ & 109.5 \\
\hline $\mathrm{H}(15 \mathrm{~B})-\mathrm{C}(15)-\mathrm{H}(15 \mathrm{C})$ & 109.5 \\
\hline $\mathrm{C}(18)-\mathrm{C}(21)-\mathrm{H}(21 \mathrm{~A})$ & 109.5 \\
\hline $\mathrm{C}(18)-\mathrm{C}(21)-\mathrm{H}(21 \mathrm{~B})$ & 109.5 \\
\hline $\mathrm{C}(18)-\mathrm{C}(21)-\mathrm{H}(21 \mathrm{C})$ & 109.5 \\
\hline $\mathrm{H}(21 \mathrm{~A})-\mathrm{C}(21)-\mathrm{H}(21 \mathrm{~B})$ & 109.5 \\
\hline $\mathrm{H}(21 \mathrm{~A})-\mathrm{C}(21)-\mathrm{H}(21 \mathrm{C})$ & 109.5 \\
\hline $\mathrm{H}(21 \mathrm{~B})-\mathrm{C}(21)-\mathrm{H}(21 \mathrm{C})$ & 109.5 \\
\hline C(18)-C(19)-H(19A) & 109.5 \\
\hline C(18)-C(19)-H(19B) & 109.5 \\
\hline C(18)-C(19)-H(19C) & 109.5 \\
\hline H(19A)-C(19)-H(19B) & 109.5 \\
\hline $\mathrm{H}(19 \mathrm{~A})-\mathrm{C}(19)-\mathrm{H}(19 \mathrm{C})$ & 109.5 \\
\hline H(19B)-C(19)-H(19C) & 109.5 \\
\hline $\mathrm{C}(18)-\mathrm{C}(20)-\mathrm{H}(20 \mathrm{~A})$ & 109.5 \\
\hline $\mathrm{C}(18)-\mathrm{C}(20)-\mathrm{H}(20 \mathrm{~B})$ & 109.5 \\
\hline $\mathrm{C}(18)-\mathrm{C}(20)-\mathrm{H}(20 \mathrm{C})$ & 109.5 \\
\hline $\mathrm{H}(20 \mathrm{~A})-\mathrm{C}(20)-\mathrm{H}(20 \mathrm{~B})$ & 109.5 \\
\hline$H(20 A)-C(20)-H(20 C)$ & 109.5 \\
\hline $\mathrm{H}(20 \mathrm{~B})-\mathrm{C}(20)-\mathrm{H}(20 \mathrm{C})$ & 109.5 \\
\hline $\mathrm{C}(2)-\mathrm{C}(3)-\mathrm{H}(3)$ & 120.3 \\
\hline $\mathrm{C}(4)-\mathrm{C}(3)-\mathrm{C}(2)$ & $119.36(19)$ \\
\hline $\mathrm{C}(4)-\mathrm{C}(3)-\mathrm{H}(3)$ & 120.3 \\
\hline $\mathrm{C}(14)-\mathrm{C}(16)-\mathrm{H}(16 \mathrm{~A})$ & 109.5 \\
\hline $\mathrm{C}(14)-\mathrm{C}(16)-\mathrm{H}(16 \mathrm{~B})$ & 109.5 \\
\hline $\mathrm{C}(14)-\mathrm{C}(16)-\mathrm{H}(16 \mathrm{C})$ & 109.5 \\
\hline $\mathrm{H}(16 \mathrm{~A})-\mathrm{C}(16)-\mathrm{H}(16 \mathrm{~B})$ & 109.5 \\
\hline $\mathrm{H}(16 \mathrm{~A})-\mathrm{C}(16)-\mathrm{H}(16 \mathrm{C})$ & 109.5 \\
\hline $\mathrm{H}(16 \mathrm{~B})-\mathrm{C}(16)-\mathrm{H}(16 \mathrm{C})$ & 109.5 \\
\hline $\mathrm{C}(2)-\mathrm{C}(1)-\mathrm{H}(1 \mathrm{~A})$ & 109.5 \\
\hline $\mathrm{C}(2)-\mathrm{C}(1)-\mathrm{H}(1 \mathrm{~B})$ & 109.5 \\
\hline $\mathrm{C}(2)-\mathrm{C}(1)-\mathrm{H}(1 \mathrm{C})$ & 109.5 \\
\hline
\end{tabular}




$\begin{array}{ll}\mathrm{H}(1 \mathrm{~A})-\mathrm{C}(1)-\mathrm{H}(1 \mathrm{~B}) & 109.5 \\ \mathrm{H}(1 \mathrm{~A})-\mathrm{C}(1)-\mathrm{H}(1 \mathrm{C}) & 109.5 \\ \mathrm{H}(1 \mathrm{~B})-\mathrm{C}(1)-\mathrm{H}(1 \mathrm{C}) & 109.5\end{array}$

Symmetry transformations used to generate equivalent atoms: 
Table S4. Anisotropic displacement parameters $\left(\AA^{2} \times 10^{3}\right)$ for $\mathbf{2 b}$. The anisotropic displacement factor exponent takes the form: $-2 \pi^{2}\left[h^{2} a^{* 2} U^{11}+\ldots+2 h k a^{*} b^{*} U^{12}\right]$

\begin{tabular}{|c|c|c|c|c|c|c|}
\hline & $\mathrm{U}^{11}$ & $\mathrm{U}^{22}$ & $\mathrm{U}^{33}$ & $\mathrm{U}^{23}$ & $\mathrm{U}^{13}$ & $\mathrm{U}^{12}$ \\
\hline $\mathrm{P}(1)$ & 11(1) & $18(1)$ & 21(1) & $1(1)$ & $-4(1)$ & $-2(1)$ \\
\hline $\mathrm{N}(1)$ & $14(1)$ & $28(1)$ & $16(1)$ & $4(1)$ & $-2(1)$ & $0(1)$ \\
\hline $\mathrm{C}(11)$ & $9(1)$ & $11(1)$ & $19(1)$ & $-2(1)$ & $0(1)$ & $3(1)$ \\
\hline$C(10)$ & $14(1)$ & $13(1)$ & $16(1)$ & $0(1)$ & $2(1)$ & $1(1)$ \\
\hline$C(12)$ & $12(1)$ & $16(1)$ & $12(1)$ & $-1(1)$ & $-1(1)$ & $4(1)$ \\
\hline$C(13)$ & $12(1)$ & $11(1)$ & $16(1)$ & $-1(1)$ & $1(1)$ & $3(1)$ \\
\hline$C(6)$ & $15(1)$ & $26(1)$ & 11(1) & $3(1)$ & $0(1)$ & 1(1) \\
\hline$C(7)$ & $13(1)$ & $22(1)$ & $13(1)$ & $-2(1)$ & $-2(1)$ & $-2(1)$ \\
\hline C(9) & $12(1)$ & $10(1)$ & $16(1)$ & $0(1)$ & $-1(1)$ & $4(1)$ \\
\hline$C(8)$ & $11(1)$ & $8(1)$ & $17(1)$ & $-1(1)$ & $-2(1)$ & $2(1)$ \\
\hline$C(18)$ & 11(1) & $19(1)$ & $20(1)$ & $-1(1)$ & $-1(1)$ & $-4(1)$ \\
\hline$C(14)$ & $17(1)$ & $16(1)$ & $13(1)$ & $-1(1)$ & $-2(1)$ & $2(1)$ \\
\hline$C(24)$ & $22(1)$ & $16(1)$ & $20(1)$ & $2(1)$ & $3(1)$ & $-1(1)$ \\
\hline$C(22)$ & $12(1)$ & $15(1)$ & $16(1)$ & $0(1)$ & $0(1)$ & $2(1)$ \\
\hline$C(2)$ & $14(1)$ & $39(1)$ & $14(1)$ & 11(1) & $-1(1)$ & $0(1)$ \\
\hline$C(5)$ & $24(1)$ & $27(1)$ & $16(1)$ & $2(1)$ & $1(1)$ & $3(1)$ \\
\hline$C(25)$ & $16(1)$ & $20(1)$ & $23(1)$ & 2(1) & $6(1)$ & $1(1)$ \\
\hline$C(17)$ & $32(1)$ & $21(1)$ & $17(1)$ & $-4(1)$ & $-3(1)$ & $-2(1)$ \\
\hline$C(23)$ & $22(1)$ & $27(1)$ & $16(1)$ & $4(1)$ & $2(1)$ & $-2(1)$ \\
\hline $\mathrm{C}(4)$ & $27(1)$ & $32(1)$ & $24(1)$ & $5(1)$ & $1(1)$ & $15(1)$ \\
\hline$C(15)$ & $24(1)$ & $34(1)$ & $15(1)$ & $-3(1)$ & $2(1)$ & $2(1)$ \\
\hline $\mathrm{C}(21)$ & 21(1) & $27(1)$ & $28(1)$ & $-8(1)$ & $-2(1)$ & $-7(1)$ \\
\hline$C(19)$ & $20(1)$ & $41(1)$ & $27(1)$ & $3(1)$ & $-2(1)$ & $-16(1)$ \\
\hline$C(20)$ & $18(1)$ & $26(1)$ & $50(2)$ & $-4(1)$ & $-12(1)$ & $2(1)$ \\
\hline C(3) & $15(1)$ & $47(2)$ & $19(1)$ & $10(1)$ & $1(1)$ & $10(1)$ \\
\hline$C(16)$ & $28(1)$ & $23(1)$ & $18(1)$ & $3(1)$ & $-2(1)$ & $5(1)$ \\
\hline$C(1)$ & $17(1)$ & $49(2)$ & $36(1)$ & $17(1)$ & $-9(1)$ & $-6(1)$ \\
\hline
\end{tabular}


Table S5. Hydrogen coordinates ( $\left.\times 10^{4}\right)$ and isotropic displacement parameters $\left(\AA^{2} \times 10^{3}\right)$ for $\mathbf{2 b}$.

\begin{tabular}{|c|c|c|c|c|}
\hline & $\mathrm{x}$ & $\mathrm{y}$ & z & $\mathrm{U}(\mathrm{eq})$ \\
\hline $\mathrm{H}(10)$ & 807 & 2655 & 6661 & 17 \\
\hline $\mathrm{H}(12)$ & 1203 & 3590 & 4872 & 16 \\
\hline $\mathrm{H}(24 \mathrm{~A})$ & 2142 & 6351 & 5271 & 29 \\
\hline $\mathrm{H}(24 \mathrm{~B})$ & 2841 & 6355 & 4844 & 29 \\
\hline $\mathrm{H}(24 \mathrm{C})$ & 2877 & 5908 & 5559 & 29 \\
\hline $\mathrm{H}(5)$ & 4015 & 786 & 6346 & 26 \\
\hline $\mathrm{H}(25 \mathrm{~A})$ & 3429 & 4064 & 5118 & 30 \\
\hline $\mathrm{H}(25 \mathrm{~B})$ & 3318 & 4477 & 4403 & 30 \\
\hline $\mathrm{H}(25 \mathrm{C})$ & 2986 & 3277 & 4637 & 30 \\
\hline $\mathrm{H}(17 \mathrm{~A})$ & 2677 & 4789 & 7319 & 35 \\
\hline $\mathrm{H}(17 \mathrm{~B})$ & 2188 & 4887 & 7934 & 35 \\
\hline $\mathrm{H}(17 \mathrm{C})$ & 1930 & 5438 & 7279 & 35 \\
\hline $\mathrm{H}(23 \mathrm{~A})$ & 1900 & 4085 & 4153 & 33 \\
\hline $\mathrm{H}(23 \mathrm{~B})$ & 2248 & 5333 & 4043 & 33 \\
\hline $\mathrm{H}(23 \mathrm{C})$ & 1529 & 5216 & 4439 & 33 \\
\hline $\mathrm{H}(4)$ & 5189 & 260 & 6570 & 33 \\
\hline $\mathrm{H}(15 \mathrm{~A})$ & 798 & 4257 & 7429 & 36 \\
\hline $\mathrm{H}(15 \mathrm{~B})$ & 1164 & 3874 & 8079 & 36 \\
\hline $\mathrm{H}(15 \mathrm{C})$ & 876 & 2913 & 7598 & 36 \\
\hline $\mathrm{H}(21 \mathrm{~A})$ & 628 & 2021 & 4597 & 38 \\
\hline $\mathrm{H}(21 \mathrm{~B})$ & -26 & 1209 & 4782 & 38 \\
\hline $\mathrm{H}(21 \mathrm{C})$ & 742 & 955 & 5070 & 38 \\
\hline $\mathrm{H}(19 \mathrm{~A})$ & 261 & 1034 & 6166 & 44 \\
\hline $\mathrm{H}(19 \mathrm{~B})$ & -499 & 1188 & 5841 & 44 \\
\hline $\mathrm{H}(19 \mathrm{C})$ & -240 & 2090 & 6367 & 44 \\
\hline $\mathrm{H}(20 \mathrm{~A})$ & -403 & 3773 & 5652 & 47 \\
\hline $\mathrm{H}(20 \mathrm{~B})$ & -719 & 2899 & 5137 & 47 \\
\hline $\mathrm{H}(20 \mathrm{C})$ & -81 & 3745 & 4952 & 47 \\
\hline $\mathrm{H}(3)$ & 6004 & 1698 & 6834 & 32 \\
\hline $\mathrm{H}(16 \mathrm{~A})$ & 2003 & 1901 & 7467 & 34 \\
\hline
\end{tabular}




\begin{tabular}{lllll}
$\mathrm{H}(16 \mathrm{~B})$ & 2255 & 2702 & 8042 & 34 \\
$\mathrm{H}(16 \mathrm{C})$ & 2722 & 2625 & 7412 & 34 \\
$\mathrm{H}(1 \mathrm{~A})$ & 5758 & 4644 & 6584 & 51 \\
$\mathrm{H}(1 \mathrm{~B})$ & 6308 & 3720 & 6861 & 51 \\
$\mathrm{H}(1 \mathrm{C})$ & 5734 & 4304 & 7317 & 51 \\
$\mathrm{H}(7)$ & $3124(10)$ & $2267(17)$ & $6267(9)$ & $16(5)$ \\
\hline
\end{tabular}


Table S6. Crystal data and structure refinement for $\mathbf{2 d}$.

Identification code

Empirical formula

Formula weight

Temperature

Wavelength

Crystal system

Space group

Unit cell dimensions

Volume

Z

Density (calculated)

Absorption coefficient

$\mathrm{F}(000)$

Crystal size

Theta range for data collection

Index ranges

Reflections collected

Independent reflections

Completeness to theta $=26.000^{\circ}$

Absorption correction

Max. and min. transmission

Refinement method

Data / restraints / parameters

Goodness-of-fit on $\mathrm{F}^{2}$

Final $\mathrm{R}$ indices [I $>2 \operatorname{sigma}(\mathrm{I})]$

$\mathrm{R}$ indices (all data)

Extinction coefficient

Largest diff. peak and hole

Twinning
MN-203

C25 H36 N O P

397.52

$100 \mathrm{~K}$

$0.71073 \AA$

Monoclinic

P 21/c

$\mathrm{a}=11.1367(15) \AA$

$\alpha=90^{\circ}$.

$\mathrm{b}=19.189(2) \AA$

$\beta=113.283(4)^{\circ}$.

$c=11.9757(17) \AA$

$2350.8(5) \AA^{3}$

4

$1.123 \mathrm{Mg} / \mathrm{m}^{3}$

$0.131 \mathrm{~mm}^{-1}$

864

$0.29 \times 0.13 \times 0.09 \mathrm{~mm}^{3}$

2.134 to $25.000^{\circ}$.

$-13<=\mathrm{h}<=13,-22<=\mathrm{k}<=22,-14<=\mathrm{l}<=14$

7678

$7678[\mathrm{R}$ (int) $=$ ?]

$99.6 \%$

Semi-empirical from equivalents

0.4901 and 0.4493

Full-matrix least-squares on $\mathrm{F}^{2}$

$7678 / 0 / 268$

1.054

$\mathrm{R} 1=0.0671, \mathrm{wR} 2=0.1756$

$\mathrm{R} 1=0.0820, \mathrm{wR} 2=0.1879$

$\mathrm{n} / \mathrm{a}$

1.890 and -0.482 e. $\AA^{-3}$

180

deg

rotational

twin 
Table S7. Atomic coordinates $\left(\times 10^{4}\right)$ and equivalent isotropic displacement parameters $\left(\AA^{2} \times 10^{3}\right)$ for $\mathbf{2 d}$. $U(e q)$ is defined as one third of the trace of the orthogonalized $U^{i j}$ tensor.

\begin{tabular}{|c|c|c|c|c|}
\hline & $\mathrm{x}$ & $\mathrm{y}$ & z & $\mathrm{U}(\mathrm{eq})$ \\
\hline $\mathrm{P}(1)$ & $7423(1)$ & $3298(1)$ & $4837(1)$ & $25(1)$ \\
\hline $\mathrm{O}(1)$ & $8581(3)$ & $5871(1)$ & $4451(2)$ & $29(1)$ \\
\hline $\mathrm{N}(1)$ & 8092(3) & $4705(1)$ & $4005(3)$ & $20(1)$ \\
\hline$C(4)$ & $6537(3)$ & $1007(2)$ & $3515(3)$ & $15(1)$ \\
\hline$C(2)$ & $5840(3)$ & 2073(2) & $4202(3)$ & $15(1)$ \\
\hline$C(3)$ & $5588(3)$ & $1417(2)$ & $3670(3)$ & $15(1)$ \\
\hline$C(5)$ & 7806(3) & $1246(2)$ & $4019(3)$ & $18(1)$ \\
\hline $\mathrm{C}(11)$ & $6146(3)$ & $304(2)$ & $2852(3)$ & $20(1)$ \\
\hline$C(7)$ & 4721(3) & $2465(2)$ & 4394(3) & $20(1)$ \\
\hline $\mathrm{C}(1)$ & $7139(3)$ & $2338(2)$ & $4584(3)$ & $18(1)$ \\
\hline$C(6)$ & $8153(3)$ & 1891(2) & $4599(3)$ & $18(1)$ \\
\hline$C(24)$ & $8380(3)$ & $5319(2)$ & $3680(3)$ & $26(1)$ \\
\hline$C(21)$ & 7930(3) & $4277(2)$ & $2056(3)$ & $26(1)$ \\
\hline$C(20)$ & 7867(3) & $4177(2)$ & $3179(3)$ & 21(1) \\
\hline$C(19)$ & $7559(3)$ & $3498(2)$ & $3514(3)$ & $20(1)$ \\
\hline$C(15)$ & $9639(3)$ & 2051(2) & $5272(3)$ & $26(1)$ \\
\hline$C(12)$ & $5155(4)$ & $431(2)$ & $1547(3)$ & $30(1)$ \\
\hline$C(13)$ & $5516(4)$ & $-162(2)$ & $3507(3)$ & $29(1)$ \\
\hline$C(23)$ & $8512(3)$ & $5466(2)$ & 2593(3) & $26(1)$ \\
\hline$C(22)$ & $8276(3)$ & $4927(2)$ & $1770(3)$ & $27(1)$ \\
\hline$C(10)$ & 3491(3) & $2009(2)$ & $4055(4)$ & $31(1)$ \\
\hline$C(25)$ & $8487(4)$ & $5723(2)$ & $5590(3)$ & $30(1)$ \\
\hline$C(8)$ & $5143(4)$ & $2650(2)$ & $5740(3)$ & $28(1)$ \\
\hline$C(16)$ & $10204(4)$ & $2452(2)$ & $4515(4)$ & $41(1)$ \\
\hline$C(9)$ & 4272(4) & $3123(2)$ & $3592(4)$ & $31(1)$ \\
\hline$C(14)$ & $7328(4)$ & $-75(2)$ & 2799(4) & $37(1)$ \\
\hline$C(17)$ & $9900(4)$ & $2465(3)$ & $6445(4)$ & $60(2)$ \\
\hline$C(18)$ & $10423(4)$ & $1376(2)$ & $5670(5)$ & $52(1)$ \\
\hline
\end{tabular}


Table S8. Bond lengths $[\AA]$ and angles $\left[{ }^{\circ}\right]$ for $\mathbf{2 d}$.

\begin{tabular}{|c|c|}
\hline $\mathrm{P}(1)-\mathrm{C}(1)$ & $1.873(3)$ \\
\hline $\mathrm{P}(1)-\mathrm{C}(19)$ & $1.694(4)$ \\
\hline $\mathrm{O}(1)-\mathrm{C}(24)$ & $1.364(4)$ \\
\hline $\mathrm{O}(1)-\mathrm{C}(25)$ & $1.437(4)$ \\
\hline $\mathrm{N}(1)-\mathrm{C}(24)$ & $1.319(5)$ \\
\hline $\mathrm{N}(1)-\mathrm{C}(20)$ & $1.368(4)$ \\
\hline$C(4)-C(3)$ & $1.386(4)$ \\
\hline $\mathrm{C}(4)-\mathrm{C}(5)$ & $1.378(4)$ \\
\hline$C(4)-C(11)$ & $1.538(4)$ \\
\hline$C(2)-C(3)$ & $1.388(4)$ \\
\hline$C(2)-C(7)$ & $1.548(4)$ \\
\hline $\mathrm{C}(2)-\mathrm{C}(1)$ & $1.428(4)$ \\
\hline $\mathrm{C}(3)-\mathrm{H}(3)$ & 0.9500 \\
\hline $\mathrm{C}(5)-\mathrm{H}(5)$ & 0.9500 \\
\hline$C(5)-C(6)$ & $1.398(5)$ \\
\hline $\mathrm{C}(11)-\mathrm{C}(12)$ & $1.535(5)$ \\
\hline$C(11)-C(13)$ & $1.530(5)$ \\
\hline$C(11)-C(14)$ & $1.527(5)$ \\
\hline$C(7)-C(10)$ & $1.539(5)$ \\
\hline$C(7)-C(8)$ & $1.532(5)$ \\
\hline$C(7)-C(9)$ & $1.546(5)$ \\
\hline$C(1)-C(6)$ & $1.414(5)$ \\
\hline$C(6)-C(15)$ & $1.558(4)$ \\
\hline$C(24)-C(23)$ & $1.395(5)$ \\
\hline $\mathrm{C}(21)-\mathrm{H}(21)$ & 0.9500 \\
\hline$C(21)-C(20)$ & $1.387(5)$ \\
\hline$C(21)-C(22)$ & $1.388(5)$ \\
\hline$C(20)-C(19)$ & $1.444(5)$ \\
\hline $\mathrm{C}(19)-\mathrm{H}(19)$ & $0.85(4)$ \\
\hline$C(15)-C(16)$ & $1.504(5)$ \\
\hline$C(15)-C(17)$ & $1.538(6)$ \\
\hline$C(15)-C(18)$ & $1.529(6)$ \\
\hline $\mathrm{C}(12)-\mathrm{H}(12 \mathrm{~A})$ & 0.9800 \\
\hline $\mathrm{C}(12)-\mathrm{H}(12 \mathrm{~B})$ & 0.9800 \\
\hline
\end{tabular}




\begin{tabular}{|c|c|}
\hline $\mathrm{C}(12)-\mathrm{H}(12 \mathrm{C})$ & 0.9800 \\
\hline $\mathrm{C}(13)-\mathrm{H}(13 \mathrm{~A})$ & 0.9800 \\
\hline C(13)-H(13B) & 0.9800 \\
\hline $\mathrm{C}(13)-\mathrm{H}(13 \mathrm{C})$ & 0.9800 \\
\hline C(23)-H(23) & 0.9500 \\
\hline$C(23)-C(22)$ & $1.380(5)$ \\
\hline $\mathrm{C}(22)-\mathrm{H}(22)$ & 0.9500 \\
\hline $\mathrm{C}(10)-\mathrm{H}(10 \mathrm{~A})$ & 0.9800 \\
\hline $\mathrm{C}(10)-\mathrm{H}(10 \mathrm{~B})$ & 0.9800 \\
\hline $\mathrm{C}(10)-\mathrm{H}(10 \mathrm{C})$ & 0.9800 \\
\hline $\mathrm{C}(25)-\mathrm{H}(25 \mathrm{~A})$ & 0.9800 \\
\hline $\mathrm{C}(25)-\mathrm{H}(25 \mathrm{~B})$ & 0.9800 \\
\hline $\mathrm{C}(25)-\mathrm{H}(25 \mathrm{C})$ & 0.9800 \\
\hline C(8)-H(8A) & 0.9800 \\
\hline $\mathrm{C}(8)-\mathrm{H}(8 \mathrm{~B})$ & 0.9800 \\
\hline $\mathrm{C}(8)-\mathrm{H}(8 \mathrm{C})$ & 0.9800 \\
\hline $\mathrm{C}(16)-\mathrm{H}(16 \mathrm{~A})$ & 0.9800 \\
\hline $\mathrm{C}(16)-\mathrm{H}(16 \mathrm{~B})$ & 0.9800 \\
\hline C(16)-H(16C) & 0.9800 \\
\hline C(9)-H(9A) & 0.9800 \\
\hline C(9)-H(9B) & 0.9800 \\
\hline C(9)-H(9C) & 0.9800 \\
\hline $\mathrm{C}(14)-\mathrm{H}(14 \mathrm{~A})$ & 0.9800 \\
\hline C(14)-H(14B) & 0.9800 \\
\hline $\mathrm{C}(14)-\mathrm{H}(14 \mathrm{C})$ & 0.9800 \\
\hline $\mathrm{C}(17)-\mathrm{H}(17 \mathrm{~A})$ & 0.9800 \\
\hline C(17)-H(17B) & 0.9800 \\
\hline C(17)-H(17C) & 0.9800 \\
\hline $\mathrm{C}(18)-\mathrm{H}(18 \mathrm{~A})$ & 0.9800 \\
\hline $\mathrm{C}(18)-\mathrm{H}(18 \mathrm{~B})$ & 0.9800 \\
\hline C(18)-H(18C) & 0.9800 \\
\hline $\mathrm{C}(19)-\mathrm{P}(1)-\mathrm{C}(1)$ & $98.27(16)$ \\
\hline$C(24)-O(1)-C(25)$ & $115.8(3)$ \\
\hline $\mathrm{C}(24)-\mathrm{N}(1)-\mathrm{C}(20)$ & $116.4(3)$ \\
\hline C(3)-C(4)-C(11) & 119.4(3) \\
\hline
\end{tabular}




\begin{tabular}{|c|c|}
\hline$C(5)-C(4)-C(3)$ & $117.2(3)$ \\
\hline $\mathrm{C}(5)-\mathrm{C}(4)-\mathrm{C}(11)$ & $123.3(3)$ \\
\hline $\mathrm{C}(3)-\mathrm{C}(2)-\mathrm{C}(7)$ & $118.7(3)$ \\
\hline$C(3)-C(2)-C(1)$ & $117.8(3)$ \\
\hline $\mathrm{C}(1)-\mathrm{C}(2)-\mathrm{C}(7)$ & $123.5(3)$ \\
\hline $\mathrm{C}(4)-\mathrm{C}(3)-\mathrm{C}(2)$ & $123.3(3)$ \\
\hline $\mathrm{C}(4)-\mathrm{C}(3)-\mathrm{H}(3)$ & 118.4 \\
\hline $\mathrm{C}(2)-\mathrm{C}(3)-\mathrm{H}(3)$ & 118.4 \\
\hline $\mathrm{C}(4)-\mathrm{C}(5)-\mathrm{H}(5)$ & 118.4 \\
\hline$C(4)-C(5)-C(6)$ & $123.1(3)$ \\
\hline $\mathrm{C}(6)-\mathrm{C}(5)-\mathrm{H}(5)$ & 118.4 \\
\hline$C(12)-C(11)-C(4)$ & $109.2(3)$ \\
\hline$C(13)-C(11)-C(4)$ & $110.0(3)$ \\
\hline $\mathrm{C}(13)-\mathrm{C}(11)-\mathrm{C}(12)$ & $108.9(3)$ \\
\hline $\mathrm{C}(14)-\mathrm{C}(11)-\mathrm{C}(4)$ & $111.5(3)$ \\
\hline $\mathrm{C}(14)-\mathrm{C}(11)-\mathrm{C}(12)$ & $108.5(3)$ \\
\hline$C(14)-C(11)-C(13)$ & $108.6(3)$ \\
\hline $\mathrm{C}(10)-\mathrm{C}(7)-\mathrm{C}(2)$ & $111.7(3)$ \\
\hline $\mathrm{C}(10)-\mathrm{C}(7)-\mathrm{C}(9)$ & $105.4(3)$ \\
\hline $\mathrm{C}(8)-\mathrm{C}(7)-\mathrm{C}(2)$ & $109.8(3)$ \\
\hline $\mathrm{C}(8)-\mathrm{C}(7)-\mathrm{C}(10)$ & $106.3(3)$ \\
\hline $\mathrm{C}(8)-\mathrm{C}(7)-\mathrm{C}(9)$ & $110.7(3)$ \\
\hline $\mathrm{C}(9)-\mathrm{C}(7)-\mathrm{C}(2)$ & $112.8(3)$ \\
\hline $\mathrm{C}(2)-\mathrm{C}(1)-\mathrm{P}(1)$ & $119.2(2)$ \\
\hline $\mathrm{C}(6)-\mathrm{C}(1)-\mathrm{P}(1)$ & $120.8(2)$ \\
\hline$C(6)-C(1)-C(2)$ & $119.4(3)$ \\
\hline$C(5)-C(6)-C(1)$ & $118.0(3)$ \\
\hline$C(5)-C(6)-C(15)$ & $117.4(3)$ \\
\hline$C(1)-C(6)-C(15)$ & $124.5(3)$ \\
\hline $\mathrm{O}(1)-\mathrm{C}(24)-\mathrm{C}(23)$ & $115.2(3)$ \\
\hline $\mathrm{N}(1)-\mathrm{C}(24)-\mathrm{O}(1)$ & 119.1(3) \\
\hline $\mathrm{N}(1)-\mathrm{C}(24)-\mathrm{C}(23)$ & $125.6(3)$ \\
\hline $\mathrm{C}(20)-\mathrm{C}(21)-\mathrm{H}(21)$ & 120.4 \\
\hline$C(20)-C(21)-C(22)$ & $119.2(3)$ \\
\hline $\mathrm{C}(22)-\mathrm{C}(21)-\mathrm{H}(21)$ & 120.4 \\
\hline $\mathrm{N}(1)-\mathrm{C}(20)-\mathrm{C}(21)$ & $122.3(3)$ \\
\hline
\end{tabular}




\begin{tabular}{|c|c|}
\hline $\mathrm{N}(1)-\mathrm{C}(20)-\mathrm{C}(19)$ & $117.6(3)$ \\
\hline $\mathrm{C}(21)-\mathrm{C}(20)-\mathrm{C}(19)$ & $120.1(3)$ \\
\hline $\mathrm{P}(1)-\mathrm{C}(19)-\mathrm{H}(19)$ & $118(3)$ \\
\hline $\mathrm{C}(20)-\mathrm{C}(19)-\mathrm{P}(1)$ & $125.7(3)$ \\
\hline $\mathrm{C}(20)-\mathrm{C}(19)-\mathrm{H}(19)$ & $117(3)$ \\
\hline$C(16)-C(15)-C(6)$ & $113.6(3)$ \\
\hline$C(16)-C(15)-C(17)$ & $108.3(4)$ \\
\hline$C(16)-C(15)-C(18)$ & 107.3(3) \\
\hline$C(17)-C(15)-C(6)$ & $110.7(3)$ \\
\hline C(18)-C(15)-C(6) & $110.7(3)$ \\
\hline C(18)-C(15)-C(17) & $105.9(4)$ \\
\hline $\mathrm{C}(11)-\mathrm{C}(12)-\mathrm{H}(12 \mathrm{~A})$ & 109.5 \\
\hline $\mathrm{C}(11)-\mathrm{C}(12)-\mathrm{H}(12 \mathrm{~B})$ & 109.5 \\
\hline $\mathrm{C}(11)-\mathrm{C}(12)-\mathrm{H}(12 \mathrm{C})$ & 109.5 \\
\hline $\mathrm{H}(12 \mathrm{~A})-\mathrm{C}(12)-\mathrm{H}(12 \mathrm{~B})$ & 109.5 \\
\hline $\mathrm{H}(12 \mathrm{~A})-\mathrm{C}(12)-\mathrm{H}(12 \mathrm{C})$ & 109.5 \\
\hline $\mathrm{H}(12 \mathrm{~B})-\mathrm{C}(12)-\mathrm{H}(12 \mathrm{C})$ & 109.5 \\
\hline $\mathrm{C}(11)-\mathrm{C}(13)-\mathrm{H}(13 \mathrm{~A})$ & 109.5 \\
\hline $\mathrm{C}(11)-\mathrm{C}(13)-\mathrm{H}(13 \mathrm{~B})$ & 109.5 \\
\hline $\mathrm{C}(11)-\mathrm{C}(13)-\mathrm{H}(13 \mathrm{C})$ & 109.5 \\
\hline $\mathrm{H}(13 \mathrm{~A})-\mathrm{C}(13)-\mathrm{H}(13 \mathrm{~B})$ & 109.5 \\
\hline $\mathrm{H}(13 \mathrm{~A})-\mathrm{C}(13)-\mathrm{H}(13 \mathrm{C})$ & 109.5 \\
\hline $\mathrm{H}(13 \mathrm{~B})-\mathrm{C}(13)-\mathrm{H}(13 \mathrm{C})$ & 109.5 \\
\hline $\mathrm{C}(24)-\mathrm{C}(23)-\mathrm{H}(23)$ & 121.5 \\
\hline $\mathrm{C}(22)-\mathrm{C}(23)-\mathrm{C}(24)$ & $117.1(3)$ \\
\hline $\mathrm{C}(22)-\mathrm{C}(23)-\mathrm{H}(23)$ & 121.5 \\
\hline $\mathrm{C}(21)-\mathrm{C}(22)-\mathrm{H}(22)$ & 120.3 \\
\hline$C(23)-C(22)-C(21)$ & $119.4(3)$ \\
\hline $\mathrm{C}(23)-\mathrm{C}(22)-\mathrm{H}(22)$ & 120.3 \\
\hline $\mathrm{C}(7)-\mathrm{C}(10)-\mathrm{H}(10 \mathrm{~A})$ & 109.5 \\
\hline $\mathrm{C}(7)-\mathrm{C}(10)-\mathrm{H}(10 \mathrm{~B})$ & 109.5 \\
\hline $\mathrm{C}(7)-\mathrm{C}(10)-\mathrm{H}(10 \mathrm{C})$ & 109.5 \\
\hline $\mathrm{H}(10 \mathrm{~A})-\mathrm{C}(10)-\mathrm{H}(10 \mathrm{~B})$ & 109.5 \\
\hline $\mathrm{H}(10 \mathrm{~A})-\mathrm{C}(10)-\mathrm{H}(10 \mathrm{C})$ & 109.5 \\
\hline $\mathrm{H}(10 \mathrm{~B})-\mathrm{C}(10)-\mathrm{H}(10 \mathrm{C})$ & 109.5 \\
\hline $\mathrm{O}(1)-\mathrm{C}(25)-\mathrm{H}(25 \mathrm{~A})$ & 109.5 \\
\hline
\end{tabular}




\begin{tabular}{|c|c|}
\hline $\mathrm{O}(1)-\mathrm{C}(25)-\mathrm{H}(25 \mathrm{~B})$ & 109.5 \\
\hline $\mathrm{O}(1)-\mathrm{C}(25)-\mathrm{H}(25 \mathrm{C})$ & 109.5 \\
\hline $\mathrm{H}(25 \mathrm{~A})-\mathrm{C}(25)-\mathrm{H}(25 \mathrm{~B})$ & 109.5 \\
\hline $\mathrm{H}(25 \mathrm{~A})-\mathrm{C}(25)-\mathrm{H}(25 \mathrm{C})$ & 109.5 \\
\hline $\mathrm{H}(25 \mathrm{~B})-\mathrm{C}(25)-\mathrm{H}(25 \mathrm{C})$ & 109.5 \\
\hline $\mathrm{C}(7)-\mathrm{C}(8)-\mathrm{H}(8 \mathrm{~A})$ & 109.5 \\
\hline $\mathrm{C}(7)-\mathrm{C}(8)-\mathrm{H}(8 \mathrm{~B})$ & 109.5 \\
\hline $\mathrm{C}(7)-\mathrm{C}(8)-\mathrm{H}(8 \mathrm{C})$ & 109.5 \\
\hline $\mathrm{H}(8 \mathrm{~A})-\mathrm{C}(8)-\mathrm{H}(8 \mathrm{~B})$ & 109.5 \\
\hline $\mathrm{H}(8 \mathrm{~A})-\mathrm{C}(8)-\mathrm{H}(8 \mathrm{C})$ & 109.5 \\
\hline $\mathrm{H}(8 \mathrm{~B})-\mathrm{C}(8)-\mathrm{H}(8 \mathrm{C})$ & 109.5 \\
\hline$C(15)-C(16)-H(16 A)$ & 109.5 \\
\hline $\mathrm{C}(15)-\mathrm{C}(16)-\mathrm{H}(16 \mathrm{~B})$ & 109.5 \\
\hline $\mathrm{C}(15)-\mathrm{C}(16)-\mathrm{H}(16 \mathrm{C})$ & 109.5 \\
\hline $\mathrm{H}(16 \mathrm{~A})-\mathrm{C}(16)-\mathrm{H}(16 \mathrm{~B})$ & 109.5 \\
\hline $\mathrm{H}(16 \mathrm{~A})-\mathrm{C}(16)-\mathrm{H}(16 \mathrm{C})$ & 109.5 \\
\hline $\mathrm{H}(16 \mathrm{~B})-\mathrm{C}(16)-\mathrm{H}(16 \mathrm{C})$ & 109.5 \\
\hline $\mathrm{C}(7)-\mathrm{C}(9)-\mathrm{H}(9 \mathrm{~A})$ & 109.5 \\
\hline $\mathrm{C}(7)-\mathrm{C}(9)-\mathrm{H}(9 \mathrm{~B})$ & 109.5 \\
\hline $\mathrm{C}(7)-\mathrm{C}(9)-\mathrm{H}(9 \mathrm{C})$ & 109.5 \\
\hline $\mathrm{H}(9 \mathrm{~A})-\mathrm{C}(9)-\mathrm{H}(9 \mathrm{~B})$ & 109.5 \\
\hline $\mathrm{H}(9 \mathrm{~A})-\mathrm{C}(9)-\mathrm{H}(9 \mathrm{C})$ & 109.5 \\
\hline $\mathrm{H}(9 \mathrm{~B})-\mathrm{C}(9)-\mathrm{H}(9 \mathrm{C})$ & 109.5 \\
\hline $\mathrm{C}(11)-\mathrm{C}(14)-\mathrm{H}(14 \mathrm{~A})$ & 109.5 \\
\hline $\mathrm{C}(11)-\mathrm{C}(14)-\mathrm{H}(14 \mathrm{~B})$ & 109.5 \\
\hline $\mathrm{C}(11)-\mathrm{C}(14)-\mathrm{H}(14 \mathrm{C})$ & 109.5 \\
\hline $\mathrm{H}(14 \mathrm{~A})-\mathrm{C}(14)-\mathrm{H}(14 \mathrm{~B})$ & 109.5 \\
\hline $\mathrm{H}(14 \mathrm{~A})-\mathrm{C}(14)-\mathrm{H}(14 \mathrm{C})$ & 109.5 \\
\hline $\mathrm{H}(14 \mathrm{~B})-\mathrm{C}(14)-\mathrm{H}(14 \mathrm{C})$ & 109.5 \\
\hline $\mathrm{C}(15)-\mathrm{C}(17)-\mathrm{H}(17 \mathrm{~A})$ & 109.5 \\
\hline $\mathrm{C}(15)-\mathrm{C}(17)-\mathrm{H}(17 \mathrm{~B})$ & 109.5 \\
\hline $\mathrm{C}(15)-\mathrm{C}(17)-\mathrm{H}(17 \mathrm{C})$ & 109.5 \\
\hline $\mathrm{H}(17 \mathrm{~A})-\mathrm{C}(17)-\mathrm{H}(17 \mathrm{~B})$ & 109.5 \\
\hline $\mathrm{H}(17 \mathrm{~A})-\mathrm{C}(17)-\mathrm{H}(17 \mathrm{C})$ & 109.5 \\
\hline $\mathrm{H}(17 \mathrm{~B})-\mathrm{C}(17)-\mathrm{H}(17 \mathrm{C})$ & 109.5 \\
\hline $\mathrm{C}(15)-\mathrm{C}(18)-\mathrm{H}(18 \mathrm{~A})$ & 109. \\
\hline
\end{tabular}




$\begin{array}{ll}\text { C(15)-C(18)-H(18B) } & 109.5 \\ \text { C(15)-C(18)-H(18C) } & 109.5 \\ \text { H(18A)-C(18)-H(18B) } & 109.5 \\ \text { H(18A)-C(18)-H(18C) } & 109.5 \\ \text { H(18B)-C(18)-H(18C) } & 109.5\end{array}$

Symmetry transformations used to generate equivalent atoms: 
Table S9. Anisotropic displacement parameters $\left(\AA^{2} \times 10^{3}\right)$ for $\mathbf{2 d}$. The anisotropic displacement factor exponent takes the form: $-2 \pi^{2}\left[h^{2} a^{* 2} U^{11}+\ldots+2 h k a^{*} b^{*} U^{12}\right]$

\begin{tabular}{|c|c|c|c|c|c|c|}
\hline & $\mathrm{U}^{11}$ & $\mathrm{U}^{22}$ & $\mathrm{U}^{33}$ & $\mathrm{U}^{23}$ & $\mathrm{U}^{13}$ & $\mathrm{U}^{12}$ \\
\hline $\mathrm{P}(1)$ & $34(1)$ & $18(1)$ & $26(1)$ & $-5(1)$ & $15(1)$ & $-6(1)$ \\
\hline $\mathrm{O}(1)$ & $42(2)$ & $18(1)$ & $33(1)$ & $-2(1)$ & $20(1)$ & $-5(1)$ \\
\hline $\mathrm{N}(1)$ & $18(1)$ & $15(2)$ & $27(2)$ & $-2(1)$ & $8(1)$ & $-3(1)$ \\
\hline$C(4)$ & $21(2)$ & $12(2)$ & $12(2)$ & $3(1)$ & $6(1)$ & $3(1)$ \\
\hline$C(2)$ & $20(2)$ & $12(2)$ & $16(2)$ & $1(1)$ & $8(1)$ & $0(1)$ \\
\hline$C(3)$ & $14(2)$ & $15(2)$ & $16(2)$ & $2(1)$ & $4(1)$ & $-1(1)$ \\
\hline$C(5)$ & $19(2)$ & $16(2)$ & $19(2)$ & $5(1)$ & $9(1)$ & $6(1)$ \\
\hline$C(11)$ & $27(2)$ & $12(2)$ & $22(2)$ & $0(1)$ & $11(2)$ & $0(1)$ \\
\hline$C(7)$ & $24(2)$ & $14(2)$ & $25(2)$ & $1(1)$ & $14(1)$ & $3(1)$ \\
\hline $\mathrm{C}(1)$ & $24(2)$ & $18(2)$ & $13(2)$ & $-4(1)$ & $9(1)$ & $-6(1)$ \\
\hline$C(6)$ & $16(2)$ & $23(2)$ & $13(2)$ & $5(1)$ & $4(1)$ & $-3(1)$ \\
\hline$C(24)$ & $24(2)$ & $27(2)$ & $27(2)$ & $-4(2)$ & $12(2)$ & $2(1)$ \\
\hline$C(21)$ & $25(2)$ & $32(2)$ & $18(2)$ & $-1(2)$ & $6(2)$ & $0(2)$ \\
\hline$C(20)$ & $18(2)$ & $23(2)$ & $22(2)$ & $-1(1)$ & $6(1)$ & $2(1)$ \\
\hline$C(19)$ & $19(2)$ & $19(2)$ & $21(2)$ & $-6(1)$ & $6(1)$ & $0(1)$ \\
\hline$C(15)$ & $15(2)$ & $32(2)$ & $25(2)$ & $4(2)$ & $2(1)$ & $-7(1)$ \\
\hline$C(12)$ & $47(2)$ & $18(2)$ & $18(2)$ & $-5(1)$ & $7(2)$ & $-7(2)$ \\
\hline$C(13)$ & $45(2)$ & $16(2)$ & $26(2)$ & $-3(1)$ & $13(2)$ & $-6(2)$ \\
\hline$C(23)$ & $28(2)$ & $21(2)$ & $29(2)$ & $8(2)$ & $11(2)$ & $0(2)$ \\
\hline$C(22)$ & $29(2)$ & $29(2)$ & $24(2)$ & $7(2)$ & $12(2)$ & $4(2)$ \\
\hline$C(10)$ & $20(2)$ & $27(2)$ & $47(2)$ & $-7(2)$ & $16(2)$ & $2(2)$ \\
\hline$C(25)$ & $38(2)$ & $26(2)$ & $28(2)$ & $-6(2)$ & $15(2)$ & $-3(2)$ \\
\hline$C(8)$ & $33(2)$ & $29(2)$ & $28(2)$ & $-3(2)$ & $19(2)$ & $5(2)$ \\
\hline$C(16)$ & $21(2)$ & $51(3)$ & $50(3)$ & $17(2)$ & $14(2)$ & $1(2)$ \\
\hline$C(9)$ & $38(2)$ & $25(2)$ & $35(2)$ & $9(2)$ & $21(2)$ & $13(2)$ \\
\hline$C(14)$ & $43(2)$ & $21(2)$ & $51(3)$ & $-12(2)$ & $23(2)$ & $3(2)$ \\
\hline$C(17)$ & $28(2)$ & $103(4)$ & $37(3)$ & $-23(3)$ & $1(2)$ & $-11(3)$ \\
\hline$C(18)$ & $27(2)$ & $43(3)$ & $68(3)$ & $14(2)$ & $-1(2)$ & $-1(2)$ \\
\hline
\end{tabular}


Table S10. Hydrogen coordinates ( $\left.\times 10^{4}\right)$ and isotropic displacement parameters $\left(\AA^{2} \times 10^{3}\right)$ for $\mathbf{2 d}$.

\begin{tabular}{|c|c|c|c|c|}
\hline & $\mathrm{x}$ & $\mathrm{y}$ & $\mathrm{z}$ & $\mathrm{U}(\mathrm{eq})$ \\
\hline $\mathrm{H}(3)$ & 4723 & 1239 & 3400 & 19 \\
\hline $\mathrm{H}(5)$ & 8478 & 958 & 3970 & 21 \\
\hline $\mathrm{H}(21)$ & 7737 & 3904 & 1489 & 31 \\
\hline $\mathrm{H}(12 \mathrm{~A})$ & 4406 & 692 & 1565 & 45 \\
\hline $\mathrm{H}(12 \mathrm{~B})$ & 4855 & -17 & 1140 & 45 \\
\hline $\mathrm{H}(12 \mathrm{C})$ & 5571 & 699 & 1101 & 45 \\
\hline $\mathrm{H}(13 \mathrm{~A})$ & 6143 & -246 & 4341 & 43 \\
\hline $\mathrm{H}(13 \mathrm{~B})$ & 5265 & -607 & 3076 & 43 \\
\hline $\mathrm{H}(13 \mathrm{C})$ & 4738 & 69 & 3522 & 43 \\
\hline $\mathrm{H}(23)$ & 8753 & 5916 & 2427 & 32 \\
\hline $\mathrm{H}(22)$ & 8351 & 5000 & 1016 & 32 \\
\hline $\mathrm{H}(10 \mathrm{~A})$ & 3127 & 1917 & 3178 & 46 \\
\hline $\mathrm{H}(10 \mathrm{~B})$ & 2840 & 2252 & 4272 & 46 \\
\hline $\mathrm{H}(10 \mathrm{C})$ & 3723 & 1566 & 4498 & 46 \\
\hline $\mathrm{H}(25 \mathrm{~A})$ & 7606 & 5553 & 5438 & 45 \\
\hline $\mathrm{H}(25 \mathrm{~B})$ & 8661 & 6148 & 6081 & 45 \\
\hline $\mathrm{H}(25 \mathrm{C})$ & 9129 & 5365 & 6026 & 45 \\
\hline $\mathrm{H}(8 \mathrm{~A})$ & 5350 & 2222 & 6226 & 42 \\
\hline $\mathrm{H}(8 \mathrm{~B})$ & 4430 & 2896 & 5856 & 42 \\
\hline $\mathrm{H}(8 \mathrm{C})$ & 5917 & 2950 & 5997 & 42 \\
\hline $\mathrm{H}(16 \mathrm{~A})$ & 10071 & 2190 & 3774 & 62 \\
\hline $\mathrm{H}(16 \mathrm{~B})$ & 11141 & 2524 & 4982 & 62 \\
\hline $\mathrm{H}(16 \mathrm{C})$ & 9767 & 2905 & 4297 & 62 \\
\hline $\mathrm{H}(9 \mathrm{~A})$ & 4983 & 3465 & 3842 & 46 \\
\hline $\mathrm{H}(9 \mathrm{~B})$ & 3509 & 3325 & 3687 & 46 \\
\hline $\mathrm{H}(9 \mathrm{C})$ & 4038 & 2997 & 2738 & 46 \\
\hline $\mathrm{H}(14 \mathrm{~A})$ & 7722 & 212 & 2356 & 56 \\
\hline $\mathrm{H}(14 \mathrm{~B})$ & 7048 & -521 & 2381 & 56 \\
\hline $\mathrm{H}(14 \mathrm{C})$ & 7971 & -157 & 3627 & 56 \\
\hline $\mathrm{H}(17 \mathrm{~A})$ & 9531 & 2934 & 6237 & 90 \\
\hline
\end{tabular}




\begin{tabular}{lclll}
$\mathrm{H}(17 \mathrm{~B})$ & 10844 & 2498 & 6918 & 90 \\
$\mathrm{H}(17 \mathrm{C})$ & 9490 & 2226 & 6927 & 90 \\
$\mathrm{H}(18 \mathrm{~A})$ & 10060 & 1093 & 6142 & 78 \\
$\mathrm{H}(18 \mathrm{~B})$ & 11338 & 1488 & 6171 & 78 \\
$\mathrm{H}(18 \mathrm{C})$ & 10375 & 1115 & 4950 & 78 \\
$\mathrm{H}(19)$ & $7450(40)$ & $3170(20)$ & $3000(40)$ & $30(11)$ \\
\hline
\end{tabular}


Table S11. Crystal data and structure refinement for $\mathbf{2 e}$.

Identification code

MN-196

Empirical formula

C30 H37 N2 O2 P

Formula weight

488.58

Temperature

$200 \mathrm{~K}$

Wavelength

Crystal system

Space group

Unit cell dimensions

$0.71073 \AA$

Orthorhombic

$\mathrm{P} 22_{1} 2_{1}$

$\mathrm{a}=10.081(3) \AA$

$\alpha=90^{\circ}$.

$\mathrm{b}=11.160(3) \AA$

$\beta=90^{\circ}$.

$\mathrm{c}=24.777(8) \AA$

Volume

$2787.6(15) \AA^{3}$

Z

Density (calculated)

4

Absorption coefficient

$1.164 \mathrm{Mg} / \mathrm{m}^{3}$

$\mathrm{F}(000)$

Crystal size

Theta range for data collection

Index ranges

Reflections collected

$0.127 \mathrm{~mm}^{-1}$

1048

$0.3 \times 0.28 \times 0.26 \mathrm{~mm}^{3}$

2.001 to $27.158^{\circ}$.

$-12<=\mathrm{h}<=10,-8<=\mathrm{k}<=14,-31<=\mathrm{l}<=20$

14089

Independent reflections

Completeness to theta $=25.242^{\circ}$

$6162[\mathrm{R}(\mathrm{int})=0.0434]$

$100.0 \%$

Absorption correction

Semi-empirical from equivalents

Max. and min. transmission

0.2612 and 0.2271

Refinement method

Data / restraints / parameters

Goodness-of-fit on $\mathrm{F}^{2}$

Full-matrix least-squares on $\mathrm{F}^{2}$

$6162 / 0 / 356$

Final $\mathrm{R}$ indices [I $>2 \operatorname{sigma}(\mathrm{I})]$

$\mathrm{R}$ indices (all data)

Absolute structure parameter

Extinction coefficient

1.028

$\mathrm{R} 1=0.0450, \mathrm{wR} 2=0.1017$

$\mathrm{R} 1=0.0588, \mathrm{wR} 2=0.1097$

$-0.05(6)$

$\mathrm{n} / \mathrm{a}$

Largest diff. peak and hole

0.219 and -0.245 e. $\AA^{-3}$ 
Table S12. Atomic coordinates ( x 10 $0^{4}$ ) and equivalent isotropic displacement parameters $\left(\AA^{2} \mathrm{x} 10^{3}\right)$ for 2e. $U(e q)$ is defined as one third of the trace of the orthogonalized $U^{i j}$ tensor.

\begin{tabular}{|c|c|c|c|c|}
\hline & $\mathrm{x}$ & $\mathrm{y}$ & z & $\mathrm{U}(\mathrm{eq})$ \\
\hline $\mathrm{P}(1)$ & $4317(1)$ & $3554(1)$ & $6343(1)$ & $34(1)$ \\
\hline $\mathrm{O}(2)$ & 1699(3) & $-2753(2)$ & $6667(1)$ & $80(1)$ \\
\hline $\mathrm{N}(1)$ & $1436(2)$ & $2814(2)$ & $6475(1)$ & $29(1)$ \\
\hline $\mathrm{O}(1)$ & $2718(3)$ & $-1357(3)$ & $6243(1)$ & $83(1)$ \\
\hline $\mathrm{N}(2)$ & $1835(3)$ & $-1704(3)$ & $6538(1)$ & $54(1)$ \\
\hline$C(3)$ & $884(3)$ & $-830(3)$ & $6752(1)$ & $40(1)$ \\
\hline$C(4)$ & $1006(3)$ & $343(3)$ & $6587(1)$ & $35(1)$ \\
\hline$C(5)$ & $90(3)$ & 1172(3) & $6767(1)$ & $32(1)$ \\
\hline$C(6)$ & 206(3) & $2440(2)$ & $6580(1)$ & $29(1)$ \\
\hline$C(7)$ & $1605(3)$ & $3941(2)$ & 6291(1) & $29(1)$ \\
\hline$C(8)$ & $2963(3)$ & $4356(2)$ & $6202(1)$ & $30(1)$ \\
\hline$C(9)$ & $5576(3)$ & $4683(2)$ & $6150(1)$ & $27(1)$ \\
\hline$C(10)$ & $6057(3)$ & $4733(2)$ & $5616(1)$ & $28(1)$ \\
\hline $\mathrm{C}(11)$ & $6598(3)$ & $5805(3)$ & $5435(1)$ & $33(1)$ \\
\hline$C(12)$ & $6755(3)$ & $6802(3)$ & $5759(1)$ & $36(1)$ \\
\hline$C(13)$ & $7350(5)$ & 7969(3) & $5548(1)$ & $58(1)$ \\
\hline$C(14)$ & $7509(17)$ & $7964(14)$ & $4949(7)$ & $92(6)$ \\
\hline$C(15)$ & $6460(30)$ & $8984(11)$ & $5687(10)$ & $149(10)$ \\
\hline$C(16)$ & $5894(3)$ & $5629(2)$ & $6515(1)$ & $29(1)$ \\
\hline$C(17)$ & $6445(3)$ & $6663(2)$ & $6299(1)$ & $36(1)$ \\
\hline$C(18)$ & $5709(3)$ & $5585(3)$ & 7134(1) & $38(1)$ \\
\hline$C(19)$ & $6720(4)$ & $6397(4)$ & $7418(1)$ & $54(1)$ \\
\hline$C(20)$ & $5944(5)$ & $4336(3)$ & $7362(1)$ & $66(1)$ \\
\hline$C(21)$ & $4326(4)$ & $6034(4)$ & $7294(1)$ & $61(1)$ \\
\hline$C(22)$ & $-904(3)$ & $3156(3)$ & $6506(1)$ & $38(1)$ \\
\hline $\mathrm{C}(23)$ & $-731(3)$ & $4306(3)$ & $6309(1)$ & $41(1)$ \\
\hline$C(24)$ & $534(3)$ & 4703(3) & $6198(1)$ & $38(1)$ \\
\hline$C(25)$ & $-92(4)$ & $-1218(3)$ & $7097(1)$ & $48(1)$ \\
\hline$C(26)$ & $-994(4)$ & $-380(3)$ & $7281(1)$ & $52(1)$ \\
\hline$C(27)$ & $-913(3)$ & $798(3)$ & $7116(1)$ & $43(1)$ \\
\hline$C(28)$ & 6033(3) & $3677(2)$ & $5210(1)$ & $34(1)$ \\
\hline
\end{tabular}




$\begin{array}{llllc}\mathrm{C}(29) & 4792(4) & 3747(3) & 4859(1) & 52(1) \\ \mathrm{C}(30) & 7247(4) & 3751(3) & 4835(1) & 56(1) \\ \mathrm{C}(31) & 6114(4) & 2437(3) & 5476(1) & 53(1) \\ \mathrm{C}(1) & 8732(18) & 8081(17) & 5794(6) & 119(9) \\ \mathrm{C}(15 \mathrm{~A}) & 7161(14) & 9029(9) & 5936(5) & 61(4) \\ \mathrm{C}(1 \mathrm{~A}) & 8842(17) & 7770(14) & 5493(12) & 143(11) \\ \mathrm{C}(14 \mathrm{~A}) & 6770(40) & 8357(18) & 5011(9) & 175(16) \\ \end{array}$


Table S13. Bond lengths $[\AA]$ and angles $\left[{ }^{\circ}\right]$ for 2 e.

\begin{tabular}{|c|c|}
\hline $\mathrm{P}(1)-\mathrm{C}(8)$ & $1.669(3)$ \\
\hline $\mathrm{P}(1)-\mathrm{C}(9)$ & $1.851(3)$ \\
\hline $\mathrm{O}(2)-\mathrm{N}(2)$ & $1.222(4)$ \\
\hline $\mathrm{N}(1)-\mathrm{C}(6)$ & $1.334(3)$ \\
\hline $\mathrm{N}(1)-\mathrm{C}(7)$ & $1.348(3)$ \\
\hline $\mathrm{O}(1)-\mathrm{N}(2)$ & $1.215(4)$ \\
\hline $\mathrm{N}(2)-\mathrm{C}(3)$ & $1.467(4)$ \\
\hline$C(3)-C(4)$ & $1.376(4)$ \\
\hline$C(3)-C(25)$ & $1.375(5)$ \\
\hline $\mathrm{C}(4)-\mathrm{H}(4)$ & 0.9500 \\
\hline$C(4)-C(5)$ & $1.381(4)$ \\
\hline$C(5)-C(6)$ & $1.494(4)$ \\
\hline$C(5)-C(27)$ & $1.395(4)$ \\
\hline$C(6)-C(22)$ & $1.386(4)$ \\
\hline$C(7)-C(8)$ & $1.461(4)$ \\
\hline$C(7)-C(24)$ & $1.394(4)$ \\
\hline $\mathrm{C}(8)-\mathrm{H}(8)$ & 0.9500 \\
\hline C(9)-C(10) & $1.409(4)$ \\
\hline$C(9)-C(16)$ & $1.427(4)$ \\
\hline$C(10)-C(11)$ & $1.390(4)$ \\
\hline $\mathrm{C}(10)-\mathrm{C}(28)$ & $1.551(4)$ \\
\hline $\mathrm{C}(11)-\mathrm{H}(11)$ & 0.9500 \\
\hline $\mathrm{C}(11)-\mathrm{C}(12)$ & $1.381(4)$ \\
\hline$C(12)-C(13)$ & $1.526(4)$ \\
\hline$C(12)-C(17)$ & $1.381(4)$ \\
\hline$C(13)-C(14)$ & $1.493(18)$ \\
\hline$C(13)-C(15)$ & $1.485(18)$ \\
\hline $\mathrm{C}(13)-\mathrm{C}(1)$ & $1.526(14)$ \\
\hline$C(13)-C(15 A)$ & $1.536(12)$ \\
\hline$C(13)-C(1 A)$ & $1.527(17)$ \\
\hline$C(13)-C(14 A)$ & $1.519(19)$ \\
\hline $\mathrm{C}(14)-\mathrm{H}(14 \mathrm{~A})$ & 0.9800 \\
\hline $\mathrm{C}(14)-\mathrm{H}(14 \mathrm{~B})$ & 0.9800 \\
\hline $\mathrm{C}(14)-\mathrm{H}(14 \mathrm{C})$ & 0.9800 \\
\hline
\end{tabular}




\begin{tabular}{|c|c|}
\hline C(15)-H(15A) & 0.9800 \\
\hline $\mathrm{C}(15)-\mathrm{H}(15 \mathrm{~B})$ & 0.9800 \\
\hline $\mathrm{C}(15)-\mathrm{H}(15 \mathrm{C})$ & 0.9800 \\
\hline$C(16)-C(17)$ & $1.389(4)$ \\
\hline$C(16)-C(18)$ & $1.547(4)$ \\
\hline C(17)-H(17) & 0.9500 \\
\hline C(18)-C(19) & $1.534(5)$ \\
\hline$C(18)-C(20)$ & $1.523(5)$ \\
\hline$C(18)-C(21)$ & $1.534(5)$ \\
\hline C(19)-H(19A) & 0.9800 \\
\hline C(19)-H(19B) & 0.9800 \\
\hline C(19)-H(19C) & 0.9800 \\
\hline $\mathrm{C}(20)-\mathrm{H}(20 \mathrm{~A})$ & 0.9800 \\
\hline $\mathrm{C}(20)-\mathrm{H}(20 \mathrm{~B})$ & 0.9800 \\
\hline $\mathrm{C}(20)-\mathrm{H}(20 \mathrm{C})$ & 0.9800 \\
\hline $\mathrm{C}(21)-\mathrm{H}(21 \mathrm{~A})$ & 0.9800 \\
\hline $\mathrm{C}(21)-\mathrm{H}(21 \mathrm{~B})$ & 0.9800 \\
\hline $\mathrm{C}(21)-\mathrm{H}(21 \mathrm{C})$ & 0.9800 \\
\hline $\mathrm{C}(22)-\mathrm{H}(22)$ & 0.9500 \\
\hline$C(22)-C(23)$ & $1.385(4)$ \\
\hline C(23)-H(23) & 0.9500 \\
\hline$C(23)-C(24)$ & $1.377(4)$ \\
\hline $\mathrm{C}(24)-\mathrm{H}(24)$ & 0.9500 \\
\hline $\mathrm{C}(25)-\mathrm{H}(25)$ & 0.9500 \\
\hline$C(25)-C(26)$ & $1.381(5)$ \\
\hline C(26)-H(26) & 0.9500 \\
\hline$C(26)-C(27)$ & $1.379(5)$ \\
\hline C(27)-H(27) & 0.9500 \\
\hline C(28)-C(29) & $1.526(4)$ \\
\hline C(28)-C(30) & $1.539(4)$ \\
\hline C(28)-C(31) & $1.535(4)$ \\
\hline C(29)-H(29A) & 0.9800 \\
\hline C(29)-H(29B) & 0.9800 \\
\hline C(29)-H(29C) & 0.9800 \\
\hline $\mathrm{C}(30)-\mathrm{H}(30 \mathrm{~A})$ & 0.9800 \\
\hline $\mathrm{C}(30)-\mathrm{H}(30 \mathrm{~B})$ & 0.9800 \\
\hline
\end{tabular}




\begin{tabular}{|c|c|}
\hline $\mathrm{C}(30)-\mathrm{H}(30 \mathrm{C})$ & 0.9800 \\
\hline $\mathrm{C}(31)-\mathrm{H}(31 \mathrm{~A})$ & 0.9800 \\
\hline $\mathrm{C}(31)-\mathrm{H}(31 \mathrm{~B})$ & 0.9800 \\
\hline $\mathrm{C}(31)-\mathrm{H}(31 \mathrm{C})$ & 0.9800 \\
\hline $\mathrm{C}(1)-\mathrm{H}(1 \mathrm{~A})$ & 0.9800 \\
\hline C(1)-H(1B) & 0.9800 \\
\hline $\mathrm{C}(1)-\mathrm{H}(1 \mathrm{C})$ & 0.9800 \\
\hline $\mathrm{C}(15 \mathrm{~A})-\mathrm{H}(15 \mathrm{D})$ & 0.9800 \\
\hline $\mathrm{C}(15 \mathrm{~A})-\mathrm{H}(15 \mathrm{E})$ & 0.9800 \\
\hline $\mathrm{C}(15 \mathrm{~A})-\mathrm{H}(15 \mathrm{~F})$ & 0.9800 \\
\hline $\mathrm{C}(1 \mathrm{~A})-\mathrm{H}(1 \mathrm{AA})$ & 0.9800 \\
\hline $\mathrm{C}(1 \mathrm{~A})-\mathrm{H}(1 \mathrm{AB})$ & 0.9800 \\
\hline $\mathrm{C}(1 \mathrm{~A})-\mathrm{H}(1 \mathrm{AC})$ & 0.9800 \\
\hline $\mathrm{C}(14 \mathrm{~A})-\mathrm{H}(14 \mathrm{D})$ & 0.9800 \\
\hline $\mathrm{C}(14 \mathrm{~A})-\mathrm{H}(14 \mathrm{E})$ & 0.9800 \\
\hline $\mathrm{C}(14 \mathrm{~A})-\mathrm{H}(14 \mathrm{~F})$ & 0.9800 \\
\hline $\mathrm{C}(8)-\mathrm{P}(1)-\mathrm{C}(9)$ & $98.17(13)$ \\
\hline $\mathrm{C}(6)-\mathrm{N}(1)-\mathrm{C}(7)$ & $118.4(2)$ \\
\hline $\mathrm{O}(2)-\mathrm{N}(2)-\mathrm{C}(3)$ & $118.0(3)$ \\
\hline $\mathrm{O}(1)-\mathrm{N}(2)-\mathrm{O}(2)$ & $123.1(3)$ \\
\hline $\mathrm{O}(1)-\mathrm{N}(2)-\mathrm{C}(3)$ & $118.9(3)$ \\
\hline $\mathrm{C}(4)-\mathrm{C}(3)-\mathrm{N}(2)$ & $117.9(3)$ \\
\hline $\mathrm{C}(25)-\mathrm{C}(3)-\mathrm{N}(2)$ & $118.9(3)$ \\
\hline$C(25)-C(3)-C(4)$ & $123.2(3)$ \\
\hline $\mathrm{C}(3)-\mathrm{C}(4)-\mathrm{H}(4)$ & 120.6 \\
\hline$C(3)-C(4)-C(5)$ & $118.8(3)$ \\
\hline $\mathrm{C}(5)-\mathrm{C}(4)-\mathrm{H}(4)$ & 120.6 \\
\hline$C(4)-C(5)-C(6)$ & $118.9(2)$ \\
\hline $\mathrm{C}(4)-\mathrm{C}(5)-\mathrm{C}(27)$ & $119.0(3)$ \\
\hline $\mathrm{C}(27)-\mathrm{C}(5)-\mathrm{C}(6)$ & $122.1(3)$ \\
\hline $\mathrm{N}(1)-\mathrm{C}(6)-\mathrm{C}(5)$ & $115.4(2)$ \\
\hline $\mathrm{N}(1)-\mathrm{C}(6)-\mathrm{C}(22)$ & $123.0(3)$ \\
\hline$C(22)-C(6)-C(5)$ & $121.6(2)$ \\
\hline $\mathrm{N}(1)-\mathrm{C}(7)-\mathrm{C}(8)$ & $117.7(2)$ \\
\hline $\mathrm{N}(1)-\mathrm{C}(7)-\mathrm{C}(24)$ & $121.8(3)$ \\
\hline
\end{tabular}




$\begin{array}{ll}\mathrm{C}(24)-\mathrm{C}(7)-\mathrm{C}(8) & 120.5(2) \\ \mathrm{P}(1)-\mathrm{C}(8)-\mathrm{H}(8) & 117.8 \\ \mathrm{C}(7)-\mathrm{C}(8)-\mathrm{P}(1) & 124.4(2) \\ \mathrm{C}(7)-\mathrm{C}(8)-\mathrm{H}(8) & 117.8 \\ \mathrm{C}(10)-\mathrm{C}(9)-\mathrm{P}(1) & 120.4(2) \\ \mathrm{C}(10)-\mathrm{C}(9)-\mathrm{C}(16) & 119.2(2) \\ \mathrm{C}(16)-\mathrm{C}(9)-\mathrm{P}(1) & 119.55(19) \\ \mathrm{C}(9)-\mathrm{C}(10)-\mathrm{C}(28) & 125.0(2) \\ \mathrm{C}(11)-\mathrm{C}(10)-\mathrm{C}(9) & 118.2(2) \\ \mathrm{C}(11)-\mathrm{C}(10)-\mathrm{C}(28) & 116.8(2) \\ \mathrm{C}(10)-\mathrm{C}(11)-\mathrm{H}(11) & 118.3 \\ \mathrm{C}(12)-\mathrm{C}(11)-\mathrm{C}(10) & 123.4(3) \\ \mathrm{C}(12)-\mathrm{C}(11)-\mathrm{H}(11) & 118.3 \\ \mathrm{C}(11)-\mathrm{C}(12)-\mathrm{C}(13) & 122.2(3) \\ \mathrm{C}(17)-\mathrm{C}(12)-\mathrm{C}(11) & 116.6(3) \\ \mathrm{C}(17)-\mathrm{C}(12)-\mathrm{C}(13) & 121.0(3) \\ \mathrm{C}(12)-\mathrm{C}(13)-\mathrm{C}(1) & 107.0(6) \\ \mathrm{C}(12)-\mathrm{C}(13)-\mathrm{C}(15 \mathrm{~A}) & 113.2(5) \\ \mathrm{C}(14)-\mathrm{C}(13)-\mathrm{C}(12) & 112.3(6) \\ \mathrm{C}(14)-\mathrm{C}(13)-\mathrm{C}(1) & 107.3(8) \\ \mathrm{C}(15)-\mathrm{C}(13)-\mathrm{C}(12) & 109.6(8) \\ \mathrm{C}(15)-\mathrm{C}(13)-\mathrm{C}(14) & 107.3(9) \\ \mathrm{C}(15)-\mathrm{C}(13)-\mathrm{C}(1) & 113.4(11) \\ \mathrm{C}(1 \mathrm{~A})-\mathrm{C}(13)-\mathrm{C}(12) & 107.0(7) \\ \mathrm{C}(1 \mathrm{~A})-\mathrm{C}(13)-\mathrm{C}(15 \mathrm{~A}) & 106.9(9) \\ \mathrm{C}(14 \mathrm{~A})-\mathrm{C}(13)-\mathrm{C}(12) & 113.1(9) \\ \mathrm{C}(14 \mathrm{~A})-\mathrm{C}(13)-\mathrm{C}(15 \mathrm{~A}) & 106.3(11) \\ \mathrm{C}(14 \mathrm{~A})-\mathrm{C}(13)-\mathrm{C}(1 \mathrm{~A}) & 110.1(14) \\ \mathrm{C}(13)-\mathrm{C}(14)-\mathrm{H}(14 \mathrm{~A}) & 109.5 \\ \mathrm{C}(13)-\mathrm{C}(14)-\mathrm{H}(14 \mathrm{~B}) & 109.5 \\ \mathrm{C}(13)-\mathrm{C}(14)-\mathrm{H}(14 \mathrm{C}) & 109.5 \\ \mathrm{H}(14 \mathrm{~A})-\mathrm{C}(14)-\mathrm{H}(14 \mathrm{~B}) & 109.5 \\ \mathrm{H}(14 \mathrm{~A})-\mathrm{C}(14)-\mathrm{H}(14 \mathrm{C}) & 109.5 \\ & \end{array}$




\begin{tabular}{|c|c|}
\hline $\mathrm{C}(13)-\mathrm{C}(15)-\mathrm{H}(15 \mathrm{C})$ & 109.5 \\
\hline $\mathrm{H}(15 \mathrm{~A})-\mathrm{C}(15)-\mathrm{H}(15 \mathrm{~B})$ & 109.5 \\
\hline $\mathrm{H}(15 \mathrm{~A})-\mathrm{C}(15)-\mathrm{H}(15 \mathrm{C})$ & 109.5 \\
\hline $\mathrm{H}(15 \mathrm{~B})-\mathrm{C}(15)-\mathrm{H}(15 \mathrm{C})$ & 109.5 \\
\hline C(9)-C(16)-C(18) & $125.4(2)$ \\
\hline$C(17)-C(16)-C(9)$ & $117.4(2)$ \\
\hline$C(17)-C(16)-C(18)$ & $117.2(2)$ \\
\hline$C(12)-C(17)-C(16)$ & $123.8(3)$ \\
\hline $\mathrm{C}(12)-\mathrm{C}(17)-\mathrm{H}(17)$ & 118.1 \\
\hline $\mathrm{C}(16)-\mathrm{C}(17)-\mathrm{H}(17)$ & 118.1 \\
\hline$C(19)-C(18)-C(16)$ & $110.9(3)$ \\
\hline $\mathrm{C}(20)-\mathrm{C}(18)-\mathrm{C}(16)$ & $112.2(2)$ \\
\hline$C(20)-C(18)-C(19)$ & $105.5(3)$ \\
\hline$C(20)-C(18)-C(21)$ & $110.1(3)$ \\
\hline$C(21)-C(18)-C(16)$ & $110.8(3)$ \\
\hline$C(21)-C(18)-C(19)$ & $107.0(3)$ \\
\hline C(18)-C(19)-H(19A) & 109.5 \\
\hline C(18)-C(19)-H(19B) & 109.5 \\
\hline C(18)-C(19)-H(19C) & 109.5 \\
\hline $\mathrm{H}(19 \mathrm{~A})-\mathrm{C}(19)-\mathrm{H}(19 \mathrm{~B})$ & 109.5 \\
\hline $\mathrm{H}(19 \mathrm{~A})-\mathrm{C}(19)-\mathrm{H}(19 \mathrm{C})$ & 109.5 \\
\hline H(19B)-C(19)-H(19C) & 109.5 \\
\hline $\mathrm{C}(18)-\mathrm{C}(20)-\mathrm{H}(20 \mathrm{~A})$ & 109.5 \\
\hline $\mathrm{C}(18)-\mathrm{C}(20)-\mathrm{H}(20 \mathrm{~B})$ & 109.5 \\
\hline C(18)-C(20)-H(20C) & 109.5 \\
\hline $\mathrm{H}(20 \mathrm{~A})-\mathrm{C}(20)-\mathrm{H}(20 \mathrm{~B})$ & 109.5 \\
\hline $\mathrm{H}(20 \mathrm{~A})-\mathrm{C}(20)-\mathrm{H}(20 \mathrm{C})$ & 109.5 \\
\hline $\mathrm{H}(20 \mathrm{~B})-\mathrm{C}(20)-\mathrm{H}(20 \mathrm{C})$ & 109.5 \\
\hline $\mathrm{C}(18)-\mathrm{C}(21)-\mathrm{H}(21 \mathrm{~A})$ & 109.5 \\
\hline $\mathrm{C}(18)-\mathrm{C}(21)-\mathrm{H}(21 \mathrm{~B})$ & 109.5 \\
\hline $\mathrm{C}(18)-\mathrm{C}(21)-\mathrm{H}(21 \mathrm{C})$ & 109.5 \\
\hline $\mathrm{H}(21 \mathrm{~A})-\mathrm{C}(21)-\mathrm{H}(21 \mathrm{~B})$ & 109.5 \\
\hline $\mathrm{H}(21 \mathrm{~A})-\mathrm{C}(21)-\mathrm{H}(21 \mathrm{C})$ & 109.5 \\
\hline $\mathrm{H}(21 \mathrm{~B})-\mathrm{C}(21)-\mathrm{H}(21 \mathrm{C})$ & 109.5 \\
\hline $\mathrm{C}(6)-\mathrm{C}(22)-\mathrm{H}(22)$ & 120.7 \\
\hline $\mathrm{C}(23)-\mathrm{C}(22)-\mathrm{C}(6)$ & $118.6(3)$ \\
\hline
\end{tabular}




\begin{tabular}{|c|c|}
\hline $\mathrm{C}(23)-\mathrm{C}(22)-\mathrm{H}(22)$ & 120.7 \\
\hline $\mathrm{C}(22)-\mathrm{C}(23)-\mathrm{H}(23)$ & 120.5 \\
\hline $\mathrm{C}(24)-\mathrm{C}(23)-\mathrm{C}(22)$ & $119.0(3)$ \\
\hline $\mathrm{C}(24)-\mathrm{C}(23)-\mathrm{H}(23)$ & 120.5 \\
\hline $\mathrm{C}(7)-\mathrm{C}(24)-\mathrm{H}(24)$ & 120.4 \\
\hline$C(23)-C(24)-C(7)$ & $119.2(3)$ \\
\hline $\mathrm{C}(23)-\mathrm{C}(24)-\mathrm{H}(24)$ & 120.4 \\
\hline $\mathrm{C}(3)-\mathrm{C}(25)-\mathrm{H}(25)$ & 121.2 \\
\hline$C(3)-C(25)-C(26)$ & $117.5(3)$ \\
\hline $\mathrm{C}(26)-\mathrm{C}(25)-\mathrm{H}(25)$ & 121.2 \\
\hline $\mathrm{C}(25)-\mathrm{C}(26)-\mathrm{H}(26)$ & 119.7 \\
\hline $\mathrm{C}(27)-\mathrm{C}(26)-\mathrm{C}(25)$ & $120.7(3)$ \\
\hline $\mathrm{C}(27)-\mathrm{C}(26)-\mathrm{H}(26)$ & 119.7 \\
\hline $\mathrm{C}(5)-\mathrm{C}(27)-\mathrm{H}(27)$ & 119.6 \\
\hline$C(26)-C(27)-C(5)$ & $120.7(3)$ \\
\hline $\mathrm{C}(26)-\mathrm{C}(27)-\mathrm{H}(27)$ & 119.6 \\
\hline$C(29)-C(28)-C(10)$ & $110.1(2)$ \\
\hline $\mathrm{C}(29)-\mathrm{C}(28)-\mathrm{C}(30)$ & $107.8(3)$ \\
\hline$C(29)-C(28)-C(31)$ & $109.6(3)$ \\
\hline $\mathrm{C}(30)-\mathrm{C}(28)-\mathrm{C}(10)$ & $109.8(3)$ \\
\hline $\mathrm{C}(31)-\mathrm{C}(28)-\mathrm{C}(10)$ & $113.9(2)$ \\
\hline $\mathrm{C}(31)-\mathrm{C}(28)-\mathrm{C}(30)$ & $105.4(3)$ \\
\hline $\mathrm{C}(28)-\mathrm{C}(29)-\mathrm{H}(29 \mathrm{~A})$ & 109.5 \\
\hline $\mathrm{C}(28)-\mathrm{C}(29)-\mathrm{H}(29 \mathrm{~B})$ & 109.5 \\
\hline C(28)-C(29)-H(29C) & 109.5 \\
\hline $\mathrm{H}(29 \mathrm{~A})-\mathrm{C}(29)-\mathrm{H}(29 \mathrm{~B})$ & 109.5 \\
\hline $\mathrm{H}(29 \mathrm{~A})-\mathrm{C}(29)-\mathrm{H}(29 \mathrm{C})$ & 109.5 \\
\hline $\mathrm{H}(29 \mathrm{~B})-\mathrm{C}(29)-\mathrm{H}(29 \mathrm{C})$ & 109.5 \\
\hline $\mathrm{C}(28)-\mathrm{C}(30)-\mathrm{H}(30 \mathrm{~A})$ & 109.5 \\
\hline $\mathrm{C}(28)-\mathrm{C}(30)-\mathrm{H}(30 \mathrm{~B})$ & 109.5 \\
\hline $\mathrm{C}(28)-\mathrm{C}(30)-\mathrm{H}(30 \mathrm{C})$ & 109.5 \\
\hline $\mathrm{H}(30 \mathrm{~A})-\mathrm{C}(30)-\mathrm{H}(30 \mathrm{~B})$ & 109.5 \\
\hline $\mathrm{H}(30 \mathrm{~A})-\mathrm{C}(30)-\mathrm{H}(30 \mathrm{C})$ & 109.5 \\
\hline $\mathrm{H}(30 \mathrm{~B})-\mathrm{C}(30)-\mathrm{H}(30 \mathrm{C})$ & 109.5 \\
\hline $\mathrm{C}(28)-\mathrm{C}(31)-\mathrm{H}(31 \mathrm{~A})$ & 109.5 \\
\hline $\mathrm{C}(28)-\mathrm{C}(31)-\mathrm{H}(31 \mathrm{~B})$ & 109.5 \\
\hline
\end{tabular}




$\begin{array}{ll}\text { C(28)-C(31)-H(31C) } & 109.5 \\ \text { H(31A)-C(31)-H(31B) } & 109.5 \\ \text { H(31A)-C(31)-H(31C) } & 109.5 \\ \text { H(31B)-C(31)-H(31C) } & 109.5 \\ \text { C(13)-C(1)-H(1A) } & 109.5 \\ \text { C(13)-C(1)-H(1B) } & 109.5 \\ \text { C(13)-C(1)-H(1C) } & 109.5 \\ \text { H(1A)-C(1)-H(1B) } & 109.5 \\ \text { H(1A)-C(1)-H(1C) } & 109.5 \\ \text { H(1B)-C(1)-H(1C) } & 109.5 \\ \text { C(13)-C(15A)-H(15D) } & 109.5 \\ \text { C(13)-C(15A)-H(15E) } & 109.5 \\ \text { C(13)-C(15A)-H(15F) } & 109.5 \\ \text { H(15D)-C(15A)-H(15E) } & 109.5 \\ \text { H(15D)-C(15A)-H(15F) } & 109.5 \\ \text { H(15E)-C(15A)-H(15F) } & 109.5 \\ \text { C(13)-C(1A)-H(1AA) } & 109.5 \\ \text { C(13)-C(1A)-H(1AB) } & 109.5 \\ \text { C(13)-C(1A)-H(1AC) } & 109.5 \\ \text { H(1AA)-C(1A)-H(1AB) } & 109.5 \\ \text { H(1AA)-C(1A)-H(1AC) } & 109.5 \\ \text { H(1AB)-C(1A)-H(1AC) } & 109.5 \\ \text { C(13)-C(14A)-H(14D) } & 109.5 \\ \text { C(13)-C(14A)-H(14E) } & 109.5 \\ \text { C(13)-C(14A)-H(14F) } & 109.5 \\ \text { H(14D)-C(14A)-H(14E) } & 109.5 \\ \text { H(14D)-C(14A)-H(14F) } & 109.5 \\ \text { H(14E)-C(14A)-H(14F) } & 109.5 \\ \end{array}$

Symmetry transformations used to generate equivalent atoms: 
Table S14. Anisotropic displacement parameters $\left(\AA^{2} \times 10^{3}\right)$ for $2 \mathrm{e}$. The anisotropic displacement factor exponent takes the form: $-2 \pi^{2}\left[h^{2} a^{* 2} U^{11}+\ldots+2 h k a^{*} b^{*} U^{12}\right]$

\begin{tabular}{|c|c|c|c|c|c|c|}
\hline & $\mathrm{U}^{11}$ & $\mathrm{U}^{22}$ & $\mathrm{U}^{33}$ & $\mathrm{U}^{23}$ & $\mathrm{U}^{13}$ & $\mathrm{U}^{12}$ \\
\hline $\mathrm{P}(1)$ & $26(1)$ & $29(1)$ & $47(1)$ & $8(1)$ & $1(1)$ & $-2(1)$ \\
\hline $\mathrm{O}(2)$ & $90(2)$ & $37(1)$ & $112(2)$ & $12(2)$ & $-15(2)$ & $5(2)$ \\
\hline $\mathrm{N}(1)$ & $26(1)$ & $30(1)$ & $31(1)$ & $-2(1)$ & $-1(1)$ & $-3(1)$ \\
\hline $\mathrm{O}(1)$ & $94(2)$ & $57(2)$ & $96(2)$ & $4(2)$ & $34(2)$ & $24(2)$ \\
\hline $\mathrm{N}(2)$ & $65(2)$ & $39(2)$ & $58(2)$ & $0(1)$ & $-16(2)$ & $6(1)$ \\
\hline$C(3)$ & $49(2)$ & $36(2)$ & $36(2)$ & $0(1)$ & $-11(1)$ & $-3(1)$ \\
\hline$C(4)$ & $35(2)$ & $38(2)$ & $31(1)$ & $2(1)$ & $-3(1)$ & $-5(1)$ \\
\hline$C(5)$ & $30(2)$ & $38(2)$ & $28(1)$ & $-1(1)$ & $-4(1)$ & $-8(1)$ \\
\hline$C(6)$ & $26(1)$ & $35(2)$ & $26(1)$ & $-4(1)$ & $0(1)$ & $-3(1)$ \\
\hline$C(7)$ & $26(1)$ & $30(1)$ & $30(1)$ & $-2(1)$ & $-3(1)$ & $-1(1)$ \\
\hline$C(8)$ & $31(2)$ & $26(1)$ & $32(1)$ & $3(1)$ & $0(1)$ & $-3(1)$ \\
\hline$C(9)$ & $21(1)$ & $25(1)$ & $35(1)$ & $5(1)$ & $-1(1)$ & $1(1)$ \\
\hline$C(10)$ & $25(1)$ & $28(1)$ & $32(1)$ & $2(1)$ & $-6(1)$ & $1(1)$ \\
\hline$C(11)$ & $33(2)$ & $35(2)$ & $32(1)$ & $5(1)$ & $-1(1)$ & $-5(1)$ \\
\hline$C(12)$ & $37(2)$ & $32(2)$ & $40(2)$ & $7(1)$ & $-6(1)$ & $-7(1)$ \\
\hline$C(13)$ & $83(3)$ & $39(2)$ & $53(2)$ & $9(2)$ & $-9(2)$ & $-23(2)$ \\
\hline$C(14)$ & $135(12)$ & $65(9)$ & $76(7)$ & $55(7)$ & $-40(8)$ & $-65(8)$ \\
\hline$C(15)$ & $270(30)$ & $38(6)$ & 133(16) & $28(8)$ & $29(16)$ & $-2(11)$ \\
\hline$C(16)$ & $24(1)$ & $29(1)$ & $34(1)$ & $0(1)$ & $-1(1)$ & $4(1)$ \\
\hline$C(17)$ & $38(2)$ & $28(1)$ & $41(2)$ & $-5(1)$ & $-5(1)$ & $-3(1)$ \\
\hline$C(18)$ & $40(2)$ & $42(2)$ & $31(1)$ & $-4(1)$ & $2(1)$ & $3(2)$ \\
\hline$C(19)$ & $50(2)$ & $73(2)$ & $37(2)$ & $-7(2)$ & $-9(2)$ & $-3(2)$ \\
\hline$C(20)$ & $108(4)$ & $56(2)$ & $35(2)$ & $8(2)$ & $-4(2)$ & $4(2)$ \\
\hline$C(21)$ & $42(2)$ & $94(3)$ & $47(2)$ & $-24(2)$ & $9(2)$ & $3(2)$ \\
\hline$C(22)$ & $24(1)$ & $47(2)$ & $43(2)$ & $-6(1)$ & $3(1)$ & $-3(1)$ \\
\hline$C(23)$ & $26(1)$ & $44(2)$ & $53(2)$ & $-1(2)$ & $-2(2)$ & $10(1)$ \\
\hline$C(24)$ & $35(2)$ & $33(2)$ & $46(2)$ & $2(1)$ & $-3(1)$ & $4(1)$ \\
\hline$C(25)$ & $61(2)$ & $43(2)$ & $41(2)$ & $11(2)$ & $-10(2)$ & $-18(2)$ \\
\hline$C(26)$ & $57(2)$ & $59(2)$ & $41(2)$ & $7(2)$ & $5(2)$ & $-21(2)$ \\
\hline$C(27)$ & $44(2)$ & $49(2)$ & $36(2)$ & $-2(1)$ & $6(1)$ & $-14(2)$ \\
\hline $\mathrm{C}(28)$ & $37(2)$ & $32(2)$ & $34(1)$ & $-5(1)$ & $-3(1)$ & $2(1)$ \\
\hline
\end{tabular}




$\begin{array}{lcccccc}\mathrm{C}(29) & 53(2) & 53(2) & 49(2) & -12(2) & -16(2) & 1(2) \\ \mathrm{C}(30) & 61(2) & 56(2) & 51(2) & -13(2) & 14(2) & 2(2) \\ \mathrm{C}(31) & 82(3) & 32(2) & 46(2) & -7(1) & -1(2) & 9(2) \\ \mathrm{C}(1) & 128(15) & 132(15) & 97(9) & 66(9) & -69(9) & -103(12) \\ \mathrm{C}(15 \mathrm{~A}) & 96(9) & 26(4) & 60(6) & 4(4) & 1(5) & -15(5) \\ \mathrm{C}(1 \mathrm{~A}) & 73(10) & 53(6) & 300(30) & 5(13) & 96(16) & -23(7) \\ \mathrm{C}(14 \mathrm{~A}) & 380(40) & 62(11) & 81(11) & 41(9) & -120(20) & -58(17) \\ \end{array}$


Table S15. Hydrogen coordinates ( $\left.\times 10^{4}\right)$ and isotropic displacement parameters $\left(\AA^{2} \times 10^{3}\right)$ for $2 \mathrm{e}$.

\begin{tabular}{|c|c|c|c|c|}
\hline & $\mathrm{x}$ & $\mathrm{y}$ & z & $\mathrm{U}(\mathrm{eq})$ \\
\hline $\mathrm{H}(4)$ & 1707 & 578 & 6354 & 42 \\
\hline $\mathrm{H}(8)$ & 3082 & 5133 & 6054 & 36 \\
\hline $\mathrm{H}(11)$ & 6875 & 5856 & 5069 & 40 \\
\hline $\mathrm{H}(14 \mathrm{~A})$ & 7981 & 7236 & 4839 & 138 \\
\hline $\mathrm{H}(14 \mathrm{~B})$ & 8019 & 8669 & 4838 & 138 \\
\hline $\mathrm{H}(14 \mathrm{C})$ & 6634 & 7982 & 4778 & 138 \\
\hline $\mathrm{H}(15 \mathrm{~A})$ & 5639 & 8922 & 5476 & 223 \\
\hline $\mathrm{H}(15 \mathrm{~B})$ & 6904 & 9742 & 5603 & 223 \\
\hline $\mathrm{H}(15 \mathrm{C})$ & 6248 & 8957 & 6073 & 223 \\
\hline $\mathrm{H}(17)$ & 6620 & 7314 & 6536 & 43 \\
\hline $\mathrm{H}(19 \mathrm{~A})$ & 6639 & 6299 & 7810 & 80 \\
\hline $\mathrm{H}(19 \mathrm{~B})$ & 6548 & 7234 & 7322 & 80 \\
\hline $\mathrm{H}(19 \mathrm{C})$ & 7618 & 6176 & 7305 & 80 \\
\hline $\mathrm{H}(20 \mathrm{~A})$ & 5853 & 4356 & 7756 & 100 \\
\hline $\mathrm{H}(20 \mathrm{~B})$ & 6839 & 4067 & 7267 & 100 \\
\hline $\mathrm{H}(20 \mathrm{C})$ & 5290 & 3780 & 7210 & 100 \\
\hline $\mathrm{H}(21 \mathrm{~A})$ & 3654 & 5474 & 7160 & 91 \\
\hline $\mathrm{H}(21 \mathrm{~B})$ & 4175 & 6827 & 7137 & 91 \\
\hline $\mathrm{H}(21 \mathrm{C})$ & 4266 & 6086 & 7688 & 91 \\
\hline $\mathrm{H}(22)$ & -1764 & 2863 & 6590 & 45 \\
\hline $\mathrm{H}(23)$ & -1474 & 4814 & 6252 & 49 \\
\hline $\mathrm{H}(24)$ & 674 & 5486 & 6060 & 46 \\
\hline $\mathrm{H}(25)$ & -146 & -2034 & 7206 & 58 \\
\hline $\mathrm{H}(26)$ & -1676 & -617 & 7523 & 63 \\
\hline $\mathrm{H}(27)$ & -1548 & 1361 & 7242 & 51 \\
\hline $\mathrm{H}(29 \mathrm{~A})$ & 4778 & 3068 & 4608 & 78 \\
\hline $\mathrm{H}(29 \mathrm{~B})$ & 4799 & 4498 & 4654 & 78 \\
\hline $\mathrm{H}(29 \mathrm{C})$ & 4002 & 3719 & 5089 & 78 \\
\hline $\mathrm{H}(30 \mathrm{~A})$ & 8056 & 3811 & 5053 & 84 \\
\hline $\mathrm{H}(30 \mathrm{~B})$ & 7170 & 4460 & 4603 & 84 \\
\hline
\end{tabular}




\begin{tabular}{lrrrr}
$\mathrm{H}(30 \mathrm{C})$ & 7289 & 3030 & 4610 & 84 \\
$\mathrm{H}(31 \mathrm{~A})$ & 5304 & 2290 & 5685 & 80 \\
$\mathrm{H}(31 \mathrm{~B})$ & 6884 & 2409 & 5717 & 80 \\
$\mathrm{H}(31 \mathrm{C})$ & 6205 & 1821 & 5197 & 80 \\
$\mathrm{H}(1 \mathrm{~A})$ & 8656 & 8294 & 6176 & 179 \\
$\mathrm{H}(1 \mathrm{~B})$ & 9230 & 8705 & 5603 & 179 \\
$\mathrm{H}(1 \mathrm{C})$ & 9200 & 7314 & 5760 & 179 \\
$\mathrm{H}(15 \mathrm{D})$ & 6238 & 9051 & 6060 & 91 \\
$\mathrm{H}(15 \mathrm{E})$ & 7372 & 9778 & 5748 & 91 \\
$\mathrm{H}(15 \mathrm{~F})$ & 7752 & 8934 & 6247 & 91 \\
$\mathrm{H}(1 \mathrm{AA})$ & 9220 & 7583 & 5848 & 214 \\
$\mathrm{H}(1 \mathrm{AB})$ & 9258 & 8497 & 5350 & 214 \\
$\mathrm{H}(1 \mathrm{AC})$ & 9006 & 7101 & 5246 & 214 \\
$\mathrm{H}(14 \mathrm{D})$ & 6838 & 7698 & 4751 & 263 \\
$\mathrm{H}(14 \mathrm{E})$ & 7254 & 9054 & 4875 & 263 \\
$\mathrm{H}(14 \mathrm{~F})$ & 5831 & 8569 & 5060 & 263 \\
\hline
\end{tabular}


Table S16. Crystal data and structure refinement for $\mathbf{3 b}$.

Identification code

$\mathrm{MN}-215$

Empirical formula

C47 H59 C12 N O P2 Ru

Formula weight

887.86

Temperature

$100.0 \mathrm{~K}$

Wavelength

$0.71073 \AA$

Crystal system

Monoclinic

Space group

Unit cell dimensions

P 21/c

$\mathrm{a}=10.8476(3) \AA$

$\alpha=90^{\circ}$.

$\mathrm{b}=18.0541(6) \AA$

$\beta=100.416(2)^{\circ}$.

$\mathrm{c}=23.2380(7) \AA$

$\gamma=90^{\circ}$.

Volume

$4476.0(2) \AA^{3}$

Z

Density (calculated)

4

Absorption coefficient

$1.318 \mathrm{Mg} / \mathrm{m}^{3}$

$\mathrm{F}(000)$

Crystal size

Theta range for data collection

Index ranges

Reflections collected

$0.576 \mathrm{~mm}^{-1}$

1856

$0.32 \times 0.28 \times 0.25 \mathrm{~mm}^{3}$

2.217 to $28.262^{\circ}$.

$-14<=\mathrm{h}<=14,-24<=\mathrm{k}<=14,-30<=1<=28$

29772

11004 [R(int) $=0.0484]$

Independent reflections

Completeness to theta $=25.242^{\circ}$

Absorption correction

Max. and min. transmission

$99.8 \%$

Refinement method

Data / restraints / parameters

Goodness-of-fit on $\mathrm{F}^{2}$

Semi-empirical from equivalents

0.4920 and 0.4417

Full-matrix least-squares on $\mathrm{F}^{2}$

11004 / 0 / 501

1.017

Final $\mathrm{R}$ indices [I $>2 \operatorname{sigma}(\mathrm{I})]$

$\mathrm{R}$ indices (all data)

Extinction coefficient

Largest diff. peak and hole SQUEEZE

$\mathrm{R} 1=0.0393, \mathrm{wR} 2=0.0792$

$\mathrm{R} 1=0.0620, \mathrm{wR} 2=0.0876$

$\mathrm{n} / \mathrm{a}$

0.736 and -0.633 e. $\AA^{-3}$

80e/uc; two THF on -1 site 
Table S17. Atomic coordinates ( $\left.\times 10^{4}\right)$ and equivalent isotropic displacement parameters $\left(\AA^{2} \times 10^{3}\right)$ for $3 \mathbf{b}$. $U(e q)$ is defined as one third of the trace of the orthogonalized $U^{i j}$ tensor.

\begin{tabular}{|c|c|c|c|c|}
\hline & $\mathrm{x}$ & $\mathrm{y}$ & $\mathrm{z}$ & $\mathrm{U}(\mathrm{eq})$ \\
\hline $\operatorname{Ru}(1)$ & 4184(1) & 6889(1) & $5859(1)$ & $9(1)$ \\
\hline $\mathrm{Cl}(2)$ & $6083(1)$ & $7016(1)$ & $6520(1)$ & $14(1)$ \\
\hline $\mathrm{Cl}(1)$ & $3031(1)$ & $6552(1)$ & 4934(1) & $14(1)$ \\
\hline $\mathrm{P}(1)$ & $4428(1)$ & $8109(1)$ & $5560(1)$ & $10(1)$ \\
\hline $\mathrm{P}(2)$ & 2766(1) & 6941(1) & $6395(1)$ & $10(1)$ \\
\hline $\mathrm{N}(1)$ & $4067(2)$ & $5737(1)$ & 6071(1) & $10(1)$ \\
\hline $\mathrm{O}(1)$ & $642(2)$ & $2215(1)$ & 6644(1) & $30(1)$ \\
\hline$C(26)$ & $5227(2)$ & $8763(1)$ & 6104(1) & $12(1)$ \\
\hline$C(3)$ & $4682(3)$ & 4471(1) & 5998(1) & $17(1)$ \\
\hline$C(38)$ & $5416(2)$ & $8089(1)$ & 4996(1) & $11(1)$ \\
\hline $\mathrm{C}(31)$ & $6119(2)$ & $9269(1)$ & 5992(1) & $15(1)$ \\
\hline$C(32)$ & $3112(2)$ & $8671(1)$ & $5185(1)$ & $10(1)$ \\
\hline$C(13)$ & 1101(2) & 8131(1) & 6421(1) & 11(1) \\
\hline$C(2)$ & $4836(2)$ & $5222(1)$ & 5902(1) & $13(1)$ \\
\hline $\mathrm{C}(9)$ & $2627(2)$ & $7908(1)$ & 7334(1) & $10(1)$ \\
\hline $\mathrm{C}(8)$ & $2058(2)$ & $7684(1)$ & 6757(1) & $10(1)$ \\
\hline$C(6)$ & $3120(2)$ & $5508(1)$ & 6351(1) & $12(1)$ \\
\hline $\mathrm{C}(4)$ & $3728(3)$ & $4237(1)$ & $6280(1)$ & $17(1)$ \\
\hline$C(22)$ & $93(2)$ & $7830(1)$ & $5917(1)$ & $13(1)$ \\
\hline$C(27)$ & $4869(2)$ & $8776(2)$ & $6650(1)$ & $17(1)$ \\
\hline$C(14)$ & $3359(2)$ & $7392(1)$ & 7814(1) & $12(1)$ \\
\hline $\mathrm{C}(41)$ & $6973(2)$ & $8030(2)$ & $4154(1)$ & $18(1)$ \\
\hline$C(7)$ & $2366(2)$ & $6076(1)$ & $6542(1)$ & $13(1)$ \\
\hline$C(15)$ & $4554(2)$ & $7053(2)$ & $7658(1)$ & $18(1)$ \\
\hline$C(12)$ & $956(2)$ & $8849(1)$ & $6622(1)$ & $13(1)$ \\
\hline$C(43)$ & $5422(2)$ & $8664(1)$ & 4593(1) & $15(1)$ \\
\hline$C(11)$ & $1643(2)$ & $9130(1)$ & 7134(1) & $13(1)$ \\
\hline $\mathrm{C}(40)$ & $6965(2)$ & $7449(2)$ & 4549(1) & $16(1)$ \\
\hline$C(10)$ & $2406(2)$ & $8638(1)$ & $7497(1)$ & $12(1)$ \\
\hline C(39) & $6200(2)$ & $7484(1)$ & $4968(1)$ & $14(1)$ \\
\hline$C(42)$ & $6196(3)$ & $8635(2)$ & 4183(1) & $18(1)$ \\
\hline
\end{tabular}




\begin{tabular}{|c|c|c|c|c|}
\hline$C(28)$ & $5367(3)$ & 9291(2) & $7068(1)$ & 21(1) \\
\hline$C(37)$ & $2436(2)$ & $8428(1)$ & $4650(1)$ & $14(1)$ \\
\hline$C(30)$ & $6620(3)$ & $9785(2)$ & $6414(1)$ & $20(1)$ \\
\hline$C(5)$ & 2943(2) & $4756(1)$ & $6458(1)$ & $15(1)$ \\
\hline$C(24)$ & $575(3)$ & $7387(2)$ & $5441(1)$ & $23(1)$ \\
\hline$C(17)$ & $3778(3)$ & $7805(2)$ & $8397(1)$ & $17(1)$ \\
\hline$C(18)$ & $1543(3)$ & 9953(1) & 7291(1) & $18(1)$ \\
\hline$C(33)$ & 2794(2) & $9347(1)$ & $5408(1)$ & $16(1)$ \\
\hline$C(16)$ & 2457(3) & $6779(1)$ & $7945(1)$ & $18(1)$ \\
\hline$C(36)$ & $1460(2)$ & $8848(1)$ & $4342(1)$ & $16(1)$ \\
\hline$C(29)$ & $6242(3)$ & $9802(2)$ & 6951(1) & $19(1)$ \\
\hline$C(1)$ & $5865(2)$ & $5487(1)$ & $5598(1)$ & $16(1)$ \\
\hline $\mathrm{C}(35)$ & $1166(3)$ & $9530(2)$ & $4569(1)$ & $18(1)$ \\
\hline$C(34)$ & 1834(3) & $9776(2)$ & $5095(1)$ & $19(1)$ \\
\hline$C(20)$ & $162(3)$ & $10178(2)$ & $7255(1)$ & $25(1)$ \\
\hline$C(23)$ & $-764(3)$ & $7321(2)$ & $6196(1)$ & $27(1)$ \\
\hline$C(21)$ & 2110(3) & $10408(2)$ & $6840(1)$ & $26(1)$ \\
\hline$C(19)$ & $2256(3)$ & $10142(2)$ & $7905(1)$ & $26(1)$ \\
\hline$C(25)$ & $-705(3)$ & $8459(2)$ & $5595(1)$ & $33(1)$ \\
\hline $\mathrm{C}(44)$ & $210(3)$ & $2864(2)$ & $6308(1)$ & $35(1)$ \\
\hline$C(45)$ & $245(4)$ & $3492(2)$ & $6726(2)$ & $42(1)$ \\
\hline$C(47)$ & 1171(4) & $2439(2)$ & $7223(1)$ & $51(1)$ \\
\hline$C(46)$ & $1283(4)$ & $3249(2)$ & $7231(2)$ & $52(1)$ \\
\hline
\end{tabular}


Table S18. Bond lengths $[\AA]$ and angles $\left[^{\circ}\right]$ for $\mathbf{3 b}$.

\begin{tabular}{|c|c|}
\hline $\mathrm{Ru}(1)-\mathrm{Cl}(2)$ & $2.3475(7)$ \\
\hline $\mathrm{Ru}(1)-\mathrm{Cl}(1)$ & $2.3627(6)$ \\
\hline $\mathrm{Ru}(1)-\mathrm{P}(1)$ & $2.3382(7)$ \\
\hline $\mathrm{Ru}(1)-\mathrm{P}(2)$ & $2.1498(6)$ \\
\hline $\mathrm{Ru}(1)-\mathrm{N}(1)$ & $2.147(2)$ \\
\hline$P(1)-C(26)$ & $1.831(3)$ \\
\hline $\mathrm{P}(1)-\mathrm{C}(38)$ & $1.838(2)$ \\
\hline $\mathrm{P}(1)-\mathrm{C}(32)$ & $1.837(3)$ \\
\hline $\mathrm{P}(2)-\mathrm{C}(8)$ & $1.825(2)$ \\
\hline $\mathrm{P}(2)-\mathrm{C}(7)$ & $1.671(3)$ \\
\hline $\mathrm{N}(1)-\mathrm{C}(2)$ & $1.354(3)$ \\
\hline $\mathrm{N}(1)-\mathrm{C}(6)$ & $1.376(3)$ \\
\hline $\mathrm{O}(1)-\mathrm{C}(44)$ & $1.438(4)$ \\
\hline $\mathrm{O}(1)-\mathrm{C}(47)$ & $1.423(4)$ \\
\hline$C(26)-C(31)$ & $1.390(3)$ \\
\hline$C(26)-C(27)$ & $1.392(3)$ \\
\hline $\mathrm{C}(3)-\mathrm{H}(3)$ & 0.9500 \\
\hline$C(3)-C(2)$ & $1.389(3)$ \\
\hline$C(3)-C(4)$ & $1.387(4)$ \\
\hline$C(38)-C(43)$ & $1.399(3)$ \\
\hline C(38)-C(39) & $1.392(3)$ \\
\hline $\mathrm{C}(31)-\mathrm{H}(31)$ & 0.9500 \\
\hline $\mathrm{C}(31)-\mathrm{C}(30)$ & $1.389(4)$ \\
\hline$C(32)-C(37)$ & $1.395(3)$ \\
\hline$C(32)-C(33)$ & $1.393(3)$ \\
\hline $\mathrm{C}(13)-\mathrm{C}(8)$ & $1.430(3)$ \\
\hline$C(13)-C(22)$ & $1.549(3)$ \\
\hline$C(13)-C(12)$ & $1.396(3)$ \\
\hline $\mathrm{C}(2)-\mathrm{C}(1)$ & $1.503(3)$ \\
\hline$C(9)-C(8)$ & $1.429(3)$ \\
\hline $\mathrm{C}(9)-\mathrm{C}(14)$ & $1.557(3)$ \\
\hline $\mathrm{C}(9)-\mathrm{C}(10)$ & $1.404(3)$ \\
\hline$C(6)-C(7)$ & $1.431(3)$ \\
\hline$C(6)-C(5)$ & $1.400(3)$ \\
\hline
\end{tabular}




\begin{tabular}{|c|c|}
\hline $\mathrm{C}(4)-\mathrm{H}(4)$ & 0.9500 \\
\hline$C(4)-C(5)$ & $1.379(3)$ \\
\hline$C(22)-C(24)$ & $1.531(3)$ \\
\hline$C(22)-C(23)$ & $1.532(4)$ \\
\hline$C(22)-C(25)$ & $1.538(4)$ \\
\hline C(27)-H(27) & 0.9500 \\
\hline C(27)-C(28) & $1.382(4)$ \\
\hline$C(14)-C(15)$ & $1.535(3)$ \\
\hline C(14)-C(17) & $1.542(3)$ \\
\hline C(14)-C(16) & $1.543(3)$ \\
\hline $\mathrm{C}(41)-\mathrm{H}(41)$ & 0.9500 \\
\hline $\mathrm{C}(41)-\mathrm{C}(40)$ & $1.394(4)$ \\
\hline $\mathrm{C}(41)-\mathrm{C}(42)$ & $1.389(4)$ \\
\hline $\mathrm{C}(7)-\mathrm{H}(7)$ & $0.96(2)$ \\
\hline $\mathrm{C}(15)-\mathrm{H}(15 \mathrm{~A})$ & 0.9800 \\
\hline $\mathrm{C}(15)-\mathrm{H}(15 \mathrm{~B})$ & 0.9800 \\
\hline $\mathrm{C}(15)-\mathrm{H}(15 \mathrm{C})$ & 0.9800 \\
\hline $\mathrm{C}(12)-\mathrm{H}(12)$ & 0.9500 \\
\hline $\mathrm{C}(12)-\mathrm{C}(11)$ & $1.382(3)$ \\
\hline C(43)-H(43) & 0.9500 \\
\hline C(43)-C(42) & $1.380(3)$ \\
\hline C(11)-C(10) & $1.391(3)$ \\
\hline C(11)-C(18) & $1.538(3)$ \\
\hline $\mathrm{C}(40)-\mathrm{H}(40)$ & 0.9500 \\
\hline C(40)-C(39) & $1.391(3)$ \\
\hline $\mathrm{C}(10)-\mathrm{H}(10)$ & 0.9500 \\
\hline C(39)-H(39) & 0.9500 \\
\hline $\mathrm{C}(42)-\mathrm{H}(42)$ & 0.9500 \\
\hline C(28)-H(28) & 0.9500 \\
\hline C(28)-C(29) & $1.385(4)$ \\
\hline C(37)-H(37) & 0.9500 \\
\hline C(37)-C(36) & $1.390(4)$ \\
\hline $\mathrm{C}(30)-\mathrm{H}(30)$ & 0.9500 \\
\hline C(30)-C(29) & $1.383(3)$ \\
\hline $\mathrm{C}(5)-\mathrm{H}(5)$ & 0.9500 \\
\hline $\mathrm{C}(24)-\mathrm{H}(24 \mathrm{~A})$ & 0.9800 \\
\hline
\end{tabular}




\begin{tabular}{|c|c|}
\hline $\mathrm{C}(24)-\mathrm{H}(24 \mathrm{~B})$ & 0.9800 \\
\hline $\mathrm{C}(24)-\mathrm{H}(24 \mathrm{C})$ & 0.9800 \\
\hline $\mathrm{C}(17)-\mathrm{H}(17 \mathrm{~A})$ & 0.9800 \\
\hline C(17)-H(17B) & 0.9800 \\
\hline C(17)-H(17C) & 0.9800 \\
\hline C(18)-C(20) & $1.540(4)$ \\
\hline$C(18)-C(21)$ & $1.544(4)$ \\
\hline C(18)-C(19) & $1.534(4)$ \\
\hline C(33)-H(33) & 0.9500 \\
\hline C(33)-C(34) & $1.393(4)$ \\
\hline $\mathrm{C}(16)-\mathrm{H}(16 \mathrm{~A})$ & 0.9800 \\
\hline $\mathrm{C}(16)-\mathrm{H}(16 \mathrm{~B})$ & 0.9800 \\
\hline $\mathrm{C}(16)-\mathrm{H}(16 \mathrm{C})$ & 0.9800 \\
\hline C(36)-H(36) & 0.9500 \\
\hline C(36)-C(35) & $1.399(4)$ \\
\hline C(29)-H(29) & 0.9500 \\
\hline $\mathrm{C}(1)-\mathrm{H}(1 \mathrm{~A})$ & 0.9800 \\
\hline C(1)-H(1B) & 0.9800 \\
\hline $\mathrm{C}(1)-\mathrm{H}(1 \mathrm{C})$ & 0.9800 \\
\hline C(35)-H(35) & 0.9500 \\
\hline C(35)-C(34) & $1.377(4)$ \\
\hline C(34)-H(34) & 0.9500 \\
\hline $\mathrm{C}(20)-\mathrm{H}(20 \mathrm{~A})$ & 0.9800 \\
\hline $\mathrm{C}(20)-\mathrm{H}(20 \mathrm{~B})$ & 0.9800 \\
\hline $\mathrm{C}(20)-\mathrm{H}(20 \mathrm{C})$ & 0.9800 \\
\hline $\mathrm{C}(23)-\mathrm{H}(23 \mathrm{~A})$ & 0.9800 \\
\hline C(23)-H(23B) & 0.9800 \\
\hline $\mathrm{C}(23)-\mathrm{H}(23 \mathrm{C})$ & 0.9800 \\
\hline $\mathrm{C}(21)-\mathrm{H}(21 \mathrm{~A})$ & 0.9800 \\
\hline $\mathrm{C}(21)-\mathrm{H}(21 \mathrm{~B})$ & 0.9800 \\
\hline $\mathrm{C}(21)-\mathrm{H}(21 \mathrm{C})$ & 0.9800 \\
\hline C(19)-H(19A) & 0.9800 \\
\hline C(19)-H(19B) & 0.9800 \\
\hline C(19)-H(19C) & 0.9800 \\
\hline $\mathrm{C}(25)-\mathrm{H}(25 \mathrm{~A})$ & 0.9800 \\
\hline $\mathrm{C}(25)-\mathrm{H}(25 \mathrm{~B})$ & 0.9800 \\
\hline
\end{tabular}




\begin{tabular}{|c|c|}
\hline $\mathrm{C}(25)-\mathrm{H}(25 \mathrm{C})$ & 0.9800 \\
\hline $\mathrm{C}(44)-\mathrm{H}(44 \mathrm{~A})$ & 0.9900 \\
\hline $\mathrm{C}(44)-\mathrm{H}(44 \mathrm{~B})$ & 0.9900 \\
\hline C(44)-C(45) & $1.489(4)$ \\
\hline $\mathrm{C}(45)-\mathrm{H}(45 \mathrm{~A})$ & 0.9900 \\
\hline $\mathrm{C}(45)-\mathrm{H}(45 \mathrm{~B})$ & 0.9900 \\
\hline$C(45)-C(46)$ & $1.536(5)$ \\
\hline $\mathrm{C}(47)-\mathrm{H}(47 \mathrm{~A})$ & 0.9900 \\
\hline $\mathrm{C}(47)-\mathrm{H}(47 \mathrm{~B})$ & 0.9900 \\
\hline C(47)-C(46) & $1.468(5)$ \\
\hline $\mathrm{C}(46)-\mathrm{H}(46 \mathrm{~A})$ & 0.9900 \\
\hline $\mathrm{C}(46)-\mathrm{H}(46 \mathrm{~B})$ & 0.9900 \\
\hline $\mathrm{Cl}(2)-\mathrm{Ru}(1)-\mathrm{Cl}(1)$ & $151.41(2)$ \\
\hline $\mathrm{P}(1)-\mathrm{Ru}(1)-\mathrm{Cl}(2)$ & $88.26(2)$ \\
\hline $\mathrm{P}(1)-\mathrm{Ru}(1)-\mathrm{Cl}(1)$ & $92.40(2)$ \\
\hline $\mathrm{P}(2)-\mathrm{Ru}(1)-\mathrm{Cl}(2)$ & $104.58(2)$ \\
\hline $\mathrm{P}(2)-\mathrm{Ru}(1)-\mathrm{Cl}(1)$ & $102.80(2)$ \\
\hline $\mathrm{P}(2)-\mathrm{Ru}(1)-\mathrm{P}(1)$ & $105.23(2)$ \\
\hline $\mathrm{N}(1)-\mathrm{Ru}(1)-\mathrm{Cl}(2)$ & $91.47(6)$ \\
\hline $\mathrm{N}(1)-\mathrm{Ru}(1)-\mathrm{Cl}(1)$ & $85.17(6)$ \\
\hline $\mathrm{N}(1)-\mathrm{Ru}(1)-\mathrm{P}(1)$ & $174.42(5)$ \\
\hline $\mathrm{N}(1)-\mathrm{Ru}(1)-\mathrm{P}(2)$ & $80.23(5)$ \\
\hline$C(26)-P(1)-R u(1)$ & $117.86(8)$ \\
\hline $\mathrm{C}(26)-\mathrm{P}(1)-\mathrm{C}(38)$ & $103.98(11)$ \\
\hline$C(26)-P(1)-C(32)$ & $101.59(11)$ \\
\hline $\mathrm{C}(38)-\mathrm{P}(1)-\mathrm{Ru}(1)$ & $107.78(8)$ \\
\hline $\mathrm{C}(32)-\mathrm{P}(1)-\mathrm{Ru}(1)$ & $122.74(8)$ \\
\hline $\mathrm{C}(32)-\mathrm{P}(1)-\mathrm{C}(38)$ & $100.17(11)$ \\
\hline $\mathrm{C}(8)-\mathrm{P}(2)-\mathrm{Ru}(1)$ & $134.73(8)$ \\
\hline $\mathrm{C}(7)-\mathrm{P}(2)-\mathrm{Ru}(1)$ & $108.50(9)$ \\
\hline $\mathrm{C}(7)-\mathrm{P}(2)-\mathrm{C}(8)$ & $116.48(11)$ \\
\hline $\mathrm{C}(2)-\mathrm{N}(1)-\mathrm{Ru}(1)$ & $122.22(16)$ \\
\hline $\mathrm{C}(2)-\mathrm{N}(1)-\mathrm{C}(6)$ & $118.9(2)$ \\
\hline $\mathrm{C}(6)-\mathrm{N}(1)-\mathrm{Ru}(1)$ & $118.71(16)$ \\
\hline $\mathrm{C}(47)-\mathrm{O}(1)-\mathrm{C}(44)$ & $108.6(2)$ \\
\hline
\end{tabular}




\begin{tabular}{|c|c|}
\hline $\mathrm{C}(31)-\mathrm{C}(26)-\mathrm{P}(1)$ & $123.59(19)$ \\
\hline$C(31)-C(26)-C(27)$ & $118.6(2)$ \\
\hline C(27)-C(26)-P(1) & $117.70(19)$ \\
\hline $\mathrm{C}(2)-\mathrm{C}(3)-\mathrm{H}(3)$ & 120.2 \\
\hline $\mathrm{C}(4)-\mathrm{C}(3)-\mathrm{H}(3)$ & 120.2 \\
\hline $\mathrm{C}(4)-\mathrm{C}(3)-\mathrm{C}(2)$ & $119.7(2)$ \\
\hline $\mathrm{C}(43)-\mathrm{C}(38)-\mathrm{P}(1)$ & $122.43(19)$ \\
\hline $\mathrm{C}(39)-\mathrm{C}(38)-\mathrm{P}(1)$ & $119.22(19)$ \\
\hline $\mathrm{C}(39)-\mathrm{C}(38)-\mathrm{C}(43)$ & $118.3(2)$ \\
\hline $\mathrm{C}(26)-\mathrm{C}(31)-\mathrm{H}(31)$ & 119.8 \\
\hline $\mathrm{C}(30)-\mathrm{C}(31)-\mathrm{C}(26)$ & $120.3(2)$ \\
\hline $\mathrm{C}(30)-\mathrm{C}(31)-\mathrm{H}(31)$ & 119.8 \\
\hline $\mathrm{C}(37)-\mathrm{C}(32)-\mathrm{P}(1)$ & $119.12(18)$ \\
\hline $\mathrm{C}(33)-\mathrm{C}(32)-\mathrm{P}(1)$ & $122.13(19)$ \\
\hline $\mathrm{C}(33)-\mathrm{C}(32)-\mathrm{C}(37)$ & $118.7(2)$ \\
\hline $\mathrm{C}(8)-\mathrm{C}(13)-\mathrm{C}(22)$ & $123.9(2)$ \\
\hline$C(12)-C(13)-C(8)$ & 117.1(2) \\
\hline$C(12)-C(13)-C(22)$ & $118.2(2)$ \\
\hline $\mathrm{N}(1)-\mathrm{C}(2)-\mathrm{C}(3)$ & $121.7(2)$ \\
\hline $\mathrm{N}(1)-\mathrm{C}(2)-\mathrm{C}(1)$ & $117.9(2)$ \\
\hline $\mathrm{C}(3)-\mathrm{C}(2)-\mathrm{C}(1)$ & $120.5(2)$ \\
\hline C(8)-C(9)-C(14) & $125.5(2)$ \\
\hline $\mathrm{C}(10)-\mathrm{C}(9)-\mathrm{C}(8)$ & $116.9(2)$ \\
\hline$C(10)-C(9)-C(14)$ & $117.3(2)$ \\
\hline $\mathrm{C}(13)-\mathrm{C}(8)-\mathrm{P}(2)$ & $119.19(18)$ \\
\hline $\mathrm{C}(9)-\mathrm{C}(8)-\mathrm{P}(2)$ & 119.61(18) \\
\hline $\mathrm{C}(9)-\mathrm{C}(8)-\mathrm{C}(13)$ & $120.0(2)$ \\
\hline $\mathrm{N}(1)-\mathrm{C}(6)-\mathrm{C}(7)$ & $116.6(2)$ \\
\hline $\mathrm{N}(1)-\mathrm{C}(6)-\mathrm{C}(5)$ & $120.9(2)$ \\
\hline$C(5)-C(6)-C(7)$ & $122.4(2)$ \\
\hline $\mathrm{C}(3)-\mathrm{C}(4)-\mathrm{H}(4)$ & 120.4 \\
\hline $\mathrm{C}(5)-\mathrm{C}(4)-\mathrm{C}(3)$ & $119.3(2)$ \\
\hline $\mathrm{C}(5)-\mathrm{C}(4)-\mathrm{H}(4)$ & 120.4 \\
\hline$C(24)-C(22)-C(13)$ & $116.2(2)$ \\
\hline$C(24)-C(22)-C(23)$ & $107.8(2)$ \\
\hline$C(24)-C(22)-C(25)$ & $105.6(2)$ \\
\hline
\end{tabular}




\begin{tabular}{|c|c|}
\hline$C(23)-C(22)-C(13)$ & $106.9(2)$ \\
\hline $\mathrm{C}(23)-\mathrm{C}(22)-\mathrm{C}(25)$ & $108.5(2)$ \\
\hline $\mathrm{C}(25)-\mathrm{C}(22)-\mathrm{C}(13)$ & $111.5(2)$ \\
\hline $\mathrm{C}(26)-\mathrm{C}(27)-\mathrm{H}(27)$ & 119.6 \\
\hline $\mathrm{C}(28)-\mathrm{C}(27)-\mathrm{C}(26)$ & $120.9(2)$ \\
\hline $\mathrm{C}(28)-\mathrm{C}(27)-\mathrm{H}(27)$ & 119.6 \\
\hline$C(15)-C(14)-C(9)$ & $114.32(19)$ \\
\hline$C(15)-C(14)-C(17)$ & $105.8(2)$ \\
\hline$C(15)-C(14)-C(16)$ & $110.7(2)$ \\
\hline$C(17)-C(14)-C(9)$ & $111.8(2)$ \\
\hline$C(17)-C(14)-C(16)$ & $105.66(19)$ \\
\hline$C(16)-C(14)-C(9)$ & $108.1(2)$ \\
\hline $\mathrm{C}(40)-\mathrm{C}(41)-\mathrm{H}(41)$ & 120.5 \\
\hline $\mathrm{C}(42)-\mathrm{C}(41)-\mathrm{H}(41)$ & 120.5 \\
\hline$C(42)-C(41)-C(40)$ & $119.0(2)$ \\
\hline $\mathrm{P}(2)-\mathrm{C}(7)-\mathrm{H}(7)$ & $125.4(16)$ \\
\hline $\mathrm{C}(6)-\mathrm{C}(7)-\mathrm{P}(2)$ & $114.98(18)$ \\
\hline $\mathrm{C}(6)-\mathrm{C}(7)-\mathrm{H}(7)$ & $119.6(16)$ \\
\hline $\mathrm{C}(14)-\mathrm{C}(15)-\mathrm{H}(15 \mathrm{~A})$ & 109.5 \\
\hline $\mathrm{C}(14)-\mathrm{C}(15)-\mathrm{H}(15 \mathrm{~B})$ & 109.5 \\
\hline $\mathrm{C}(14)-\mathrm{C}(15)-\mathrm{H}(15 \mathrm{C})$ & 109.5 \\
\hline $\mathrm{H}(15 \mathrm{~A})-\mathrm{C}(15)-\mathrm{H}(15 \mathrm{~B})$ & 109.5 \\
\hline $\mathrm{H}(15 \mathrm{~A})-\mathrm{C}(15)-\mathrm{H}(15 \mathrm{C})$ & 109.5 \\
\hline $\mathrm{H}(15 \mathrm{~B})-\mathrm{C}(15)-\mathrm{H}(15 \mathrm{C})$ & 109.5 \\
\hline $\mathrm{C}(13)-\mathrm{C}(12)-\mathrm{H}(12)$ & 118.2 \\
\hline$C(11)-C(12)-C(13)$ & $123.7(2)$ \\
\hline $\mathrm{C}(11)-\mathrm{C}(12)-\mathrm{H}(12)$ & 118.2 \\
\hline $\mathrm{C}(38)-\mathrm{C}(43)-\mathrm{H}(43)$ & 119.7 \\
\hline$C(42)-C(43)-C(38)$ & $120.7(2)$ \\
\hline $\mathrm{C}(42)-\mathrm{C}(43)-\mathrm{H}(43)$ & 119.7 \\
\hline$C(12)-C(11)-C(10)$ & $117.1(2)$ \\
\hline$C(12)-C(11)-C(18)$ & $120.6(2)$ \\
\hline$C(10)-C(11)-C(18)$ & $122.3(2)$ \\
\hline $\mathrm{C}(41)-\mathrm{C}(40)-\mathrm{H}(40)$ & 120.0 \\
\hline$C(39)-C(40)-C(41)$ & $120.1(2)$ \\
\hline $\mathrm{C}(39)-\mathrm{C}(40)-\mathrm{H}(40)$ & 120.0 \\
\hline
\end{tabular}




\begin{tabular}{|c|c|}
\hline $\mathrm{C}(9)-\mathrm{C}(10)-\mathrm{H}(10)$ & 118.4 \\
\hline$C(11)-C(10)-C(9)$ & $123.3(2)$ \\
\hline $\mathrm{C}(11)-\mathrm{C}(10)-\mathrm{H}(10)$ & 118.4 \\
\hline $\mathrm{C}(38)-\mathrm{C}(39)-\mathrm{H}(39)$ & 119.5 \\
\hline $\mathrm{C}(40)-\mathrm{C}(39)-\mathrm{C}(38)$ & $121.0(2)$ \\
\hline $\mathrm{C}(40)-\mathrm{C}(39)-\mathrm{H}(39)$ & 119.5 \\
\hline $\mathrm{C}(41)-\mathrm{C}(42)-\mathrm{H}(42)$ & 119.6 \\
\hline $\mathrm{C}(43)-\mathrm{C}(42)-\mathrm{C}(41)$ & $120.9(2)$ \\
\hline $\mathrm{C}(43)-\mathrm{C}(42)-\mathrm{H}(42)$ & 119.6 \\
\hline $\mathrm{C}(27)-\mathrm{C}(28)-\mathrm{H}(28)$ & 119.8 \\
\hline$C(27)-C(28)-C(29)$ & $120.3(2)$ \\
\hline $\mathrm{C}(29)-\mathrm{C}(28)-\mathrm{H}(28)$ & 119.8 \\
\hline $\mathrm{C}(32)-\mathrm{C}(37)-\mathrm{H}(37)$ & 119.5 \\
\hline$C(36)-C(37)-C(32)$ & $121.1(2)$ \\
\hline $\mathrm{C}(36)-\mathrm{C}(37)-\mathrm{H}(37)$ & 119.5 \\
\hline $\mathrm{C}(31)-\mathrm{C}(30)-\mathrm{H}(30)$ & 119.7 \\
\hline$C(29)-C(30)-C(31)$ & $120.6(2)$ \\
\hline $\mathrm{C}(29)-\mathrm{C}(30)-\mathrm{H}(30)$ & 119.7 \\
\hline $\mathrm{C}(6)-\mathrm{C}(5)-\mathrm{H}(5)$ & 120.2 \\
\hline$C(4)-C(5)-C(6)$ & $119.6(2)$ \\
\hline $\mathrm{C}(4)-\mathrm{C}(5)-\mathrm{H}(5)$ & 120.2 \\
\hline $\mathrm{C}(22)-\mathrm{C}(24)-\mathrm{H}(24 \mathrm{~A})$ & 109.5 \\
\hline $\mathrm{C}(22)-\mathrm{C}(24)-\mathrm{H}(24 \mathrm{~B})$ & 109.5 \\
\hline $\mathrm{C}(22)-\mathrm{C}(24)-\mathrm{H}(24 \mathrm{C})$ & 109.5 \\
\hline $\mathrm{H}(24 \mathrm{~A})-\mathrm{C}(24)-\mathrm{H}(24 \mathrm{~B})$ & 109.5 \\
\hline $\mathrm{H}(24 \mathrm{~A})-\mathrm{C}(24)-\mathrm{H}(24 \mathrm{C})$ & 109.5 \\
\hline $\mathrm{H}(24 \mathrm{~B})-\mathrm{C}(24)-\mathrm{H}(24 \mathrm{C})$ & 109.5 \\
\hline $\mathrm{C}(14)-\mathrm{C}(17)-\mathrm{H}(17 \mathrm{~A})$ & 109.5 \\
\hline $\mathrm{C}(14)-\mathrm{C}(17)-\mathrm{H}(17 \mathrm{~B})$ & 109.5 \\
\hline $\mathrm{C}(14)-\mathrm{C}(17)-\mathrm{H}(17 \mathrm{C})$ & 109.5 \\
\hline $\mathrm{H}(17 \mathrm{~A})-\mathrm{C}(17)-\mathrm{H}(17 \mathrm{~B})$ & 109.5 \\
\hline $\mathrm{H}(17 \mathrm{~A})-\mathrm{C}(17)-\mathrm{H}(17 \mathrm{C})$ & 109.5 \\
\hline $\mathrm{H}(17 \mathrm{~B})-\mathrm{C}(17)-\mathrm{H}(17 \mathrm{C})$ & 109.5 \\
\hline $\mathrm{C}(11)-\mathrm{C}(18)-\mathrm{C}(20)$ & $110.6(2)$ \\
\hline $\mathrm{C}(11)-\mathrm{C}(18)-\mathrm{C}(21)$ & $107.4(2)$ \\
\hline$C(20)-C(18)-C(21)$ & $109.1(2)$ \\
\hline
\end{tabular}




\begin{tabular}{|c|c|}
\hline $\mathrm{C}(19)-\mathrm{C}(18)-\mathrm{C}(11)$ & $112.9(2)$ \\
\hline$C(19)-C(18)-C(20)$ & $108.2(2)$ \\
\hline $\mathrm{C}(19)-\mathrm{C}(18)-\mathrm{C}(21)$ & $108.6(2)$ \\
\hline $\mathrm{C}(32)-\mathrm{C}(33)-\mathrm{H}(33)$ & 119.8 \\
\hline $\mathrm{C}(34)-\mathrm{C}(33)-\mathrm{C}(32)$ & $120.4(2)$ \\
\hline $\mathrm{C}(34)-\mathrm{C}(33)-\mathrm{H}(33)$ & 119.8 \\
\hline $\mathrm{C}(14)-\mathrm{C}(16)-\mathrm{H}(16 \mathrm{~A})$ & 109.5 \\
\hline $\mathrm{C}(14)-\mathrm{C}(16)-\mathrm{H}(16 \mathrm{~B})$ & 109.5 \\
\hline $\mathrm{C}(14)-\mathrm{C}(16)-\mathrm{H}(16 \mathrm{C})$ & 109.5 \\
\hline $\mathrm{H}(16 \mathrm{~A})-\mathrm{C}(16)-\mathrm{H}(16 \mathrm{~B})$ & 109.5 \\
\hline $\mathrm{H}(16 \mathrm{~A})-\mathrm{C}(16)-\mathrm{H}(16 \mathrm{C})$ & 109.5 \\
\hline $\mathrm{H}(16 \mathrm{~B})-\mathrm{C}(16)-\mathrm{H}(16 \mathrm{C})$ & 109.5 \\
\hline $\mathrm{C}(37)-\mathrm{C}(36)-\mathrm{H}(36)$ & 120.3 \\
\hline C(37)-C(36)-C(35) & $119.4(2)$ \\
\hline $\mathrm{C}(35)-\mathrm{C}(36)-\mathrm{H}(36)$ & 120.3 \\
\hline $\mathrm{C}(28)-\mathrm{C}(29)-\mathrm{H}(29)$ & 120.4 \\
\hline$C(30)-C(29)-C(28)$ & $119.2(2)$ \\
\hline $\mathrm{C}(30)-\mathrm{C}(29)-\mathrm{H}(29)$ & 120.4 \\
\hline $\mathrm{C}(2)-\mathrm{C}(1)-\mathrm{H}(1 \mathrm{~A})$ & 109.5 \\
\hline $\mathrm{C}(2)-\mathrm{C}(1)-\mathrm{H}(1 \mathrm{~B})$ & 109.5 \\
\hline $\mathrm{C}(2)-\mathrm{C}(1)-\mathrm{H}(1 \mathrm{C})$ & 109.5 \\
\hline $\mathrm{H}(1 \mathrm{~A})-\mathrm{C}(1)-\mathrm{H}(1 \mathrm{~B})$ & 109.5 \\
\hline $\mathrm{H}(1 \mathrm{~A})-\mathrm{C}(1)-\mathrm{H}(1 \mathrm{C})$ & 109.5 \\
\hline $\mathrm{H}(1 \mathrm{~B})-\mathrm{C}(1)-\mathrm{H}(1 \mathrm{C})$ & 109.5 \\
\hline $\mathrm{C}(36)-\mathrm{C}(35)-\mathrm{H}(35)$ & 120.0 \\
\hline $\mathrm{C}(34)-\mathrm{C}(35)-\mathrm{C}(36)$ & $119.9(2)$ \\
\hline $\mathrm{C}(34)-\mathrm{C}(35)-\mathrm{H}(35)$ & 120.0 \\
\hline $\mathrm{C}(33)-\mathrm{C}(34)-\mathrm{H}(34)$ & 119.7 \\
\hline $\mathrm{C}(35)-\mathrm{C}(34)-\mathrm{C}(33)$ & $120.5(2)$ \\
\hline $\mathrm{C}(35)-\mathrm{C}(34)-\mathrm{H}(34)$ & 119.7 \\
\hline $\mathrm{C}(18)-\mathrm{C}(20)-\mathrm{H}(20 \mathrm{~A})$ & 109.5 \\
\hline $\mathrm{C}(18)-\mathrm{C}(20)-\mathrm{H}(20 \mathrm{~B})$ & 109.5 \\
\hline $\mathrm{C}(18)-\mathrm{C}(20)-\mathrm{H}(20 \mathrm{C})$ & 109.5 \\
\hline $\mathrm{H}(20 \mathrm{~A})-\mathrm{C}(20)-\mathrm{H}(20 \mathrm{~B})$ & 109.5 \\
\hline $\mathrm{H}(20 \mathrm{~A})-\mathrm{C}(20)-\mathrm{H}(20 \mathrm{C})$ & 109.5 \\
\hline $\mathrm{H}(20 \mathrm{~B})-\mathrm{C}(20)-\mathrm{H}(20 \mathrm{C})$ & 109.5 \\
\hline
\end{tabular}




\begin{tabular}{|c|c|}
\hline $\mathrm{C}(22)-\mathrm{C}(23)-\mathrm{H}(23 \mathrm{~A})$ & 109.5 \\
\hline $\mathrm{C}(22)-\mathrm{C}(23)-\mathrm{H}(23 \mathrm{~B})$ & 109.5 \\
\hline $\mathrm{C}(22)-\mathrm{C}(23)-\mathrm{H}(23 \mathrm{C})$ & 109.5 \\
\hline $\mathrm{H}(23 \mathrm{~A})-\mathrm{C}(23)-\mathrm{H}(23 \mathrm{~B})$ & 109.5 \\
\hline $\mathrm{H}(23 \mathrm{~A})-\mathrm{C}(23)-\mathrm{H}(23 \mathrm{C})$ & 109.5 \\
\hline $\mathrm{H}(23 \mathrm{~B})-\mathrm{C}(23)-\mathrm{H}(23 \mathrm{C})$ & 109.5 \\
\hline $\mathrm{C}(18)-\mathrm{C}(21)-\mathrm{H}(21 \mathrm{~A})$ & 109.5 \\
\hline $\mathrm{C}(18)-\mathrm{C}(21)-\mathrm{H}(21 \mathrm{~B})$ & 109.5 \\
\hline $\mathrm{C}(18)-\mathrm{C}(21)-\mathrm{H}(21 \mathrm{C})$ & 109.5 \\
\hline $\mathrm{H}(21 \mathrm{~A})-\mathrm{C}(21)-\mathrm{H}(21 \mathrm{~B})$ & 109.5 \\
\hline $\mathrm{H}(21 \mathrm{~A})-\mathrm{C}(21)-\mathrm{H}(21 \mathrm{C})$ & 109.5 \\
\hline $\mathrm{H}(21 \mathrm{~B})-\mathrm{C}(21)-\mathrm{H}(21 \mathrm{C})$ & 109.5 \\
\hline $\mathrm{C}(18)-\mathrm{C}(19)-\mathrm{H}(19 \mathrm{~A})$ & 109.5 \\
\hline C(18)-C(19)-H(19B) & 109.5 \\
\hline C(18)-C(19)-H(19C) & 109.5 \\
\hline $\mathrm{H}(19 \mathrm{~A})-\mathrm{C}(19)-\mathrm{H}(19 \mathrm{~B})$ & 109.5 \\
\hline $\mathrm{H}(19 \mathrm{~A})-\mathrm{C}(19)-\mathrm{H}(19 \mathrm{C})$ & 109.5 \\
\hline $\mathrm{H}(19 \mathrm{~B})-\mathrm{C}(19)-\mathrm{H}(19 \mathrm{C})$ & 109.5 \\
\hline $\mathrm{C}(22)-\mathrm{C}(25)-\mathrm{H}(25 \mathrm{~A})$ & 109.5 \\
\hline $\mathrm{C}(22)-\mathrm{C}(25)-\mathrm{H}(25 \mathrm{~B})$ & 109.5 \\
\hline $\mathrm{C}(22)-\mathrm{C}(25)-\mathrm{H}(25 \mathrm{C})$ & 109.5 \\
\hline $\mathrm{H}(25 \mathrm{~A})-\mathrm{C}(25)-\mathrm{H}(25 \mathrm{~B})$ & 109.5 \\
\hline $\mathrm{H}(25 \mathrm{~A})-\mathrm{C}(25)-\mathrm{H}(25 \mathrm{C})$ & 109.5 \\
\hline $\mathrm{H}(25 \mathrm{~B})-\mathrm{C}(25)-\mathrm{H}(25 \mathrm{C})$ & 109.5 \\
\hline $\mathrm{O}(1)-\mathrm{C}(44)-\mathrm{H}(44 \mathrm{~A})$ & 110.2 \\
\hline $\mathrm{O}(1)-\mathrm{C}(44)-\mathrm{H}(44 \mathrm{~B})$ & 110.2 \\
\hline $\mathrm{O}(1)-\mathrm{C}(44)-\mathrm{C}(45)$ & $107.4(2)$ \\
\hline $\mathrm{H}(44 \mathrm{~A})-\mathrm{C}(44)-\mathrm{H}(44 \mathrm{~B})$ & 108.5 \\
\hline $\mathrm{C}(45)-\mathrm{C}(44)-\mathrm{H}(44 \mathrm{~A})$ & 110.2 \\
\hline $\mathrm{C}(45)-\mathrm{C}(44)-\mathrm{H}(44 \mathrm{~B})$ & 110.2 \\
\hline $\mathrm{C}(44)-\mathrm{C}(45)-\mathrm{H}(45 \mathrm{~A})$ & 111.4 \\
\hline $\mathrm{C}(44)-\mathrm{C}(45)-\mathrm{H}(45 \mathrm{~B})$ & 111.4 \\
\hline$C(44)-C(45)-C(46)$ & $102.1(3)$ \\
\hline $\mathrm{H}(45 \mathrm{~A})-\mathrm{C}(45)-\mathrm{H}(45 \mathrm{~B})$ & 109.2 \\
\hline $\mathrm{C}(46)-\mathrm{C}(45)-\mathrm{H}(45 \mathrm{~A})$ & 111.4 \\
\hline $\mathrm{C}(46)-\mathrm{C}(45)-\mathrm{H}(45 \mathrm{~B})$ & 111.4 \\
\hline
\end{tabular}




$\begin{array}{ll}\mathrm{O}(1)-\mathrm{C}(47)-\mathrm{H}(47 \mathrm{~A}) & 110.1 \\ \mathrm{O}(1)-\mathrm{C}(47)-\mathrm{H}(47 \mathrm{~B}) & 110.1 \\ \mathrm{O}(1)-\mathrm{C}(47)-\mathrm{C}(46) & 108.2(3) \\ \mathrm{H}(47 \mathrm{~A})-\mathrm{C}(47)-\mathrm{H}(47 \mathrm{~B}) & 108.4 \\ \mathrm{C}(46)-\mathrm{C}(47)-\mathrm{H}(47 \mathrm{~A}) & 110.1 \\ \mathrm{C}(46)-\mathrm{C}(47)-\mathrm{H}(47 \mathrm{~B}) & 110.1 \\ \mathrm{C}(45)-\mathrm{C}(46)-\mathrm{H}(46 \mathrm{~A}) & 111.1 \\ \mathrm{C}(45)-\mathrm{C}(46)-\mathrm{H}(46 \mathrm{~B}) & 111.1 \\ \mathrm{C}(47)-\mathrm{C}(46)-\mathrm{C}(45) & 103.1(3) \\ \mathrm{C}(47)-\mathrm{C}(46)-\mathrm{H}(46 \mathrm{~A}) & 111.1 \\ \mathrm{C}(47)-\mathrm{C}(46)-\mathrm{H}(46 \mathrm{~B}) & 111.1 \\ \mathrm{H}(46 \mathrm{~A})-\mathrm{C}(46)-\mathrm{H}(46 \mathrm{~B}) & 109.1\end{array}$

Symmetry transformations used to generate equivalent atoms: 
Table S19. Anisotropic displacement parameters $\left(\AA^{2} \times 10^{3}\right)$ for $\mathbf{3 b}$. The anisotropic displacement factor exponent takes the form: $-2 \pi^{2}\left[h^{2} a^{* 2} U^{11}+\ldots+2 h \mathrm{k}^{*} b^{*} U^{12}\right]$

\begin{tabular}{|c|c|c|c|c|c|c|}
\hline & $\mathrm{U}^{11}$ & $\mathrm{U}^{22}$ & $\mathrm{U}^{33}$ & $\mathrm{U}^{23}$ & $\mathrm{U}^{13}$ & $\mathrm{U}^{12}$ \\
\hline $\mathrm{Ru}(1)$ & $9(1)$ & $9(1)$ & $8(1)$ & $0(1)$ & 2(1) & 1(1) \\
\hline $\mathrm{Cl}(2)$ & $10(1)$ & $20(1)$ & $13(1)$ & $0(1)$ & $0(1)$ & 1(1) \\
\hline $\mathrm{Cl}(1)$ & $17(1)$ & $13(1)$ & $11(1)$ & $-2(1)$ & $1(1)$ & 1(1) \\
\hline $\mathrm{P}(1)$ & $10(1)$ & $10(1)$ & $9(1)$ & $0(1)$ & $2(1)$ & $-1(1)$ \\
\hline $\mathrm{P}(2)$ & $10(1)$ & $10(1)$ & $10(1)$ & $-1(1)$ & $3(1)$ & $0(1)$ \\
\hline $\mathrm{N}(1)$ & $13(1)$ & $9(1)$ & $7(1)$ & $-1(1)$ & $1(1)$ & 1(1) \\
\hline $\mathrm{O}(1)$ & $36(1)$ & $30(1)$ & $22(1)$ & $-9(1)$ & $-3(1)$ & $10(1)$ \\
\hline$C(26)$ & 11(1) & $10(1)$ & $13(1)$ & $1(1)$ & $0(1)$ & $0(1)$ \\
\hline$C(3)$ & $22(2)$ & $16(1)$ & $13(1)$ & $1(1)$ & $1(1)$ & $10(1)$ \\
\hline$C(38)$ & $10(1)$ & $15(1)$ & $10(1)$ & $-4(1)$ & $3(1)$ & $-6(1)$ \\
\hline$C(31)$ & $15(1)$ & $16(1)$ & $14(1)$ & $1(1)$ & $4(1)$ & $-3(1)$ \\
\hline$C(32)$ & $9(1)$ & 11(1) & $11(1)$ & $2(1)$ & $3(1)$ & $-1(1)$ \\
\hline$C(13)$ & $11(1)$ & $13(1)$ & $10(1)$ & $1(1)$ & $3(1)$ & $0(1)$ \\
\hline$C(2)$ & $17(1)$ & $14(1)$ & $8(1)$ & $-1(1)$ & $1(1)$ & $4(1)$ \\
\hline$C(9)$ & $7(1)$ & 11(1) & $10(1)$ & $1(1)$ & $2(1)$ & $-1(1)$ \\
\hline$C(8)$ & $10(1)$ & $6(1)$ & $13(1)$ & $1(1)$ & $4(1)$ & $-1(1)$ \\
\hline$C(6)$ & $14(1)$ & $13(1)$ & $8(1)$ & $-2(1)$ & $0(1)$ & $-1(1)$ \\
\hline$C(4)$ & $24(2)$ & $9(1)$ & $16(1)$ & $0(1)$ & $-2(1)$ & 2(1) \\
\hline$C(22)$ & $10(1)$ & $14(1)$ & $14(1)$ & $0(1)$ & $-1(1)$ & 1(1) \\
\hline$C(27)$ & $18(1)$ & 19(1) & $14(1)$ & $0(1)$ & $5(1)$ & $-9(1)$ \\
\hline$C(14)$ & $13(1)$ & $12(1)$ & $9(1)$ & $1(1)$ & $0(1)$ & $2(1)$ \\
\hline$C(41)$ & $15(1)$ & $27(2)$ & $12(1)$ & $-7(1)$ & $4(1)$ & $-8(1)$ \\
\hline$C(7)$ & $14(1)$ & $12(1)$ & $12(1)$ & $0(1)$ & $4(1)$ & $-3(1)$ \\
\hline$C(15)$ & $17(1)$ & $22(2)$ & $14(1)$ & $2(1)$ & $3(1)$ & $5(1)$ \\
\hline$C(12)$ & $12(1)$ & $12(1)$ & $14(1)$ & $2(1)$ & $-1(1)$ & $6(1)$ \\
\hline$C(43)$ & $18(1)$ & $13(1)$ & $14(1)$ & $-2(1)$ & $2(1)$ & $-2(1)$ \\
\hline$C(11)$ & $14(1)$ & $12(1)$ & $12(1)$ & $-1(1)$ & $2(1)$ & 2(1) \\
\hline$C(40)$ & $10(1)$ & $22(1)$ & $16(1)$ & $-7(1)$ & $0(1)$ & $-1(1)$ \\
\hline$C(10)$ & $13(1)$ & $15(1)$ & $7(1)$ & $-2(1)$ & $-1(1)$ & $-1(1)$ \\
\hline C(39) & $12(1)$ & $17(1)$ & $13(1)$ & $-1(1)$ & $-1(1)$ & $-2(1)$ \\
\hline $\mathrm{C}(42)$ & $22(2)$ & $20(1)$ & 11(1) & $0(1)$ & $4(1)$ & $-9(1)$ \\
\hline
\end{tabular}




\begin{tabular}{|c|c|c|c|c|c|c|}
\hline$C(28)$ & $24(2)$ & $25(2)$ & $13(1)$ & $-3(1)$ & $3(1)$ & $-4(1)$ \\
\hline$C(37)$ & $16(1)$ & $12(1)$ & $13(1)$ & $1(1)$ & $2(1)$ & $2(1)$ \\
\hline$C(30)$ & $21(2)$ & $20(1)$ & $21(2)$ & $0(1)$ & $5(1)$ & $-8(1)$ \\
\hline$C(5)$ & $16(1)$ & $13(1)$ & $15(1)$ & $-1(1)$ & $2(1)$ & $-3(1)$ \\
\hline$C(24)$ & $17(2)$ & $38(2)$ & $13(1)$ & $-7(1)$ & $1(1)$ & $-2(1)$ \\
\hline$C(17)$ & $21(2)$ & $18(1)$ & 11(1) & $1(1)$ & $-2(1)$ & $3(1)$ \\
\hline$C(18)$ & $22(2)$ & $13(1)$ & $16(1)$ & $-2(1)$ & $-1(1)$ & $4(1)$ \\
\hline$C(33)$ & $18(1)$ & $16(1)$ & $14(1)$ & $-1(1)$ & $2(1)$ & $2(1)$ \\
\hline$C(16)$ & $24(2)$ & $17(1)$ & $15(1)$ & $2(1)$ & $5(1)$ & $-2(1)$ \\
\hline$C(36)$ & $17(1)$ & $17(1)$ & $12(1)$ & $1(1)$ & $1(1)$ & $-2(1)$ \\
\hline$C(29)$ & $22(2)$ & $17(1)$ & $16(1)$ & $-3(1)$ & $-1(1)$ & $-6(1)$ \\
\hline$C(1)$ & $18(1)$ & $17(1)$ & $13(1)$ & $0(1)$ & $4(1)$ & $5(1)$ \\
\hline$C(35)$ & $17(1)$ & $21(2)$ & $15(1)$ & $6(1)$ & $1(1)$ & $7(1)$ \\
\hline$C(34)$ & $21(2)$ & $16(1)$ & $21(1)$ & $-2(1)$ & $4(1)$ & $4(1)$ \\
\hline$C(20)$ & $29(2)$ & $16(1)$ & $30(2)$ & $-4(1)$ & $2(1)$ & $8(1)$ \\
\hline$C(23)$ & $23(2)$ & $41(2)$ & $17(2)$ & $-5(1)$ & $3(1)$ & $-15(1)$ \\
\hline$C(21)$ & $35(2)$ & $13(1)$ & $29(2)$ & $1(1)$ & $7(1)$ & $2(1)$ \\
\hline$C(19)$ & $39(2)$ & $12(1)$ & $24(2)$ & $-8(1)$ & $-2(1)$ & 1(1) \\
\hline$C(25)$ & $30(2)$ & $21(2)$ & $37(2)$ & $-6(1)$ & $-23(2)$ & $7(1)$ \\
\hline $\mathrm{C}(44)$ & $36(2)$ & $44(2)$ & $22(2)$ & $0(1)$ & $0(2)$ & $22(2)$ \\
\hline$C(45)$ & $52(2)$ & $26(2)$ & $46(2)$ & $3(2)$ & $5(2)$ & $-9(2)$ \\
\hline $\mathrm{C}(47)$ & $82(3)$ & $34(2)$ & $27(2)$ & $-12(2)$ & $-18(2)$ & $5(2)$ \\
\hline$C(46)$ & $86(3)$ & $32(2)$ & $32(2)$ & $6(2)$ & $-8(2)$ & $-20(2)$ \\
\hline
\end{tabular}


Table S20. Hydrogen coordinates ( $\left.\times 10^{4}\right)$ and isotropic displacement parameters $\left(\AA^{2} \times 10^{3}\right)$ for $\mathbf{3 b}$.

\begin{tabular}{|c|c|c|c|c|}
\hline & $\mathrm{x}$ & $\mathrm{y}$ & $\mathrm{z}$ & $\mathrm{U}(\mathrm{eq})$ \\
\hline $\mathrm{H}(3)$ & 5227 & 4119 & 5871 & 20 \\
\hline $\mathrm{H}(31)$ & 6388 & 9263 & 5625 & 18 \\
\hline $\mathrm{H}(4)$ & 3617 & 3725 & 6349 & 20 \\
\hline $\mathrm{H}(27)$ & 4274 & 8426 & 6736 & 20 \\
\hline $\mathrm{H}(41)$ & 7501 & 8012 & 3870 & 22 \\
\hline $\mathrm{H}(15 \mathrm{~A})$ & 4330 & 6725 & 7319 & 27 \\
\hline $\mathrm{H}(15 \mathrm{~B})$ & 4995 & 6769 & 7992 & 27 \\
\hline $\mathrm{H}(15 \mathrm{C})$ & 5100 & 7450 & 7563 & 27 \\
\hline $\mathrm{H}(12)$ & 351 & 9161 & 6395 & 16 \\
\hline $\mathrm{H}(43)$ & 4888 & 9080 & 4602 & 18 \\
\hline $\mathrm{H}(40)$ & 7484 & 7028 & 4531 & 20 \\
\hline $\mathrm{H}(10)$ & 2798 & 8803 & 7874 & 14 \\
\hline $\mathrm{H}(39)$ & 6211 & 7090 & 5239 & 17 \\
\hline $\mathrm{H}(42)$ & 6198 & 9035 & 3917 & 21 \\
\hline $\mathrm{H}(28)$ & 5107 & 9295 & 7437 & 25 \\
\hline $\mathrm{H}(37)$ & 2645 & 7968 & 4494 & 17 \\
\hline $\mathrm{H}(30)$ & 7227 & 10130 & 6332 & 24 \\
\hline $\mathrm{H}(5)$ & 2286 & 4604 & 6652 & 18 \\
\hline $\mathrm{H}(24 \mathrm{~A})$ & 1224 & 7673 & 5297 & 34 \\
\hline $\mathrm{H}(24 \mathrm{~B})$ & -120 & 7288 & 5118 & 34 \\
\hline $\mathrm{H}(24 \mathrm{C})$ & 930 & 6917 & 5605 & 34 \\
\hline $\mathrm{H}(17 \mathrm{~A})$ & 4352 & 8207 & 8341 & 25 \\
\hline $\mathrm{H}(17 \mathrm{~B})$ & 4207 & 7459 & 8692 & 25 \\
\hline $\mathrm{H}(17 \mathrm{C})$ & 3042 & 8012 & 8530 & 25 \\
\hline $\mathrm{H}(33)$ & 3235 & 9517 & 5775 & 19 \\
\hline $\mathrm{H}(16 \mathrm{~A})$ & 1717 & 7006 & 8060 & 28 \\
\hline $\mathrm{H}(16 \mathrm{~B})$ & 2884 & 6466 & 8265 & 28 \\
\hline $\mathrm{H}(16 \mathrm{C})$ & 2198 & 6476 & 7594 & 28 \\
\hline $\mathrm{H}(36)$ & 998 & 8673 & 3981 & 19 \\
\hline H(29) & 6579 & 10159 & 7236 & 22 \\
\hline
\end{tabular}




\begin{tabular}{|c|c|c|c|c|}
\hline $\mathrm{H}(1 \mathrm{~A})$ & 5506 & 5792 & 5260 & 24 \\
\hline $\mathrm{H}(1 \mathrm{~B})$ & 6295 & 5059 & 5465 & 24 \\
\hline $\mathrm{H}(1 \mathrm{C})$ & 6466 & 5782 & 5870 & 24 \\
\hline $\mathrm{H}(35)$ & 506 & 9823 & 4360 & 21 \\
\hline $\mathrm{H}(34)$ & 1638 & 10242 & 5245 & 23 \\
\hline $\mathrm{H}(20 \mathrm{~A})$ & -301 & 10086 & 6859 & 38 \\
\hline $\mathrm{H}(20 \mathrm{~B})$ & 115 & 10706 & 7347 & 38 \\
\hline $\mathrm{H}(20 \mathrm{C})$ & -207 & 9887 & 7537 & 38 \\
\hline $\mathrm{H}(23 \mathrm{~A})$ & -276 & 6904 & 6388 & 41 \\
\hline $\mathrm{H}(23 \mathrm{~B})$ & -1439 & 7134 & 5893 & 41 \\
\hline $\mathrm{H}(23 \mathrm{C})$ & -1126 & 7600 & 6487 & 41 \\
\hline $\mathrm{H}(21 \mathrm{~A})$ & 2999 & 10281 & 6871 & 39 \\
\hline $\mathrm{H}(21 \mathrm{~B})$ & 2029 & 10937 & 6917 & 39 \\
\hline $\mathrm{H}(21 \mathrm{C})$ & 1664 & 10292 & 6444 & 39 \\
\hline $\mathrm{H}(19 \mathrm{~A})$ & 1905 & 9857 & 8197 & 39 \\
\hline $\mathrm{H}(19 \mathrm{~B})$ & 2175 & 10672 & 7979 & 39 \\
\hline $\mathrm{H}(19 \mathrm{C})$ & 3143 & 10016 & 7933 & 39 \\
\hline $\mathrm{H}(25 \mathrm{~A})$ & -1161 & 8710 & 5866 & 49 \\
\hline $\mathrm{H}(25 \mathrm{~B})$ & -1305 & 8253 & 5268 & 49 \\
\hline $\mathrm{H}(25 \mathrm{C})$ & -158 & 8815 & 5445 & 49 \\
\hline $\mathrm{H}(44 \mathrm{~A})$ & -655 & 2787 & 6093 & 42 \\
\hline $\mathrm{H}(44 \mathrm{~B})$ & 759 & 2969 & 6021 & 42 \\
\hline $\mathrm{H}(45 \mathrm{~A})$ & -567 & 3551 & 6858 & 50 \\
\hline $\mathrm{H}(45 \mathrm{~B})$ & 463 & 3963 & 6550 & 50 \\
\hline $\mathrm{H}(47 \mathrm{~A})$ & 2006 & 2210 & 7344 & 61 \\
\hline $\mathrm{H}(47 \mathrm{~B})$ & 626 & 2277 & 7499 & 61 \\
\hline $\mathrm{H}(46 \mathrm{~A})$ & 2119 & 3406 & 7162 & 62 \\
\hline $\mathrm{H}(46 \mathrm{~B})$ & 1142 & 3455 & 7608 & 62 \\
\hline $\mathrm{H}(7)$ & $1680(20)$ & $5942(14)$ & $6726(11)$ & $13(7)$ \\
\hline
\end{tabular}


Table S21. Crystal data and structure refinement for $\mathbf{4 b}$.

Identification code

$\mathrm{MN}-225 \mathrm{~A}$

Empirical formula

C43 H51 Cl2 N P2 Ru

Formula weight

815.76

Temperature

$100.0 \mathrm{~K}$

Wavelength

$0.71073 \AA$

Crystal system

Space group

Unit cell dimensions

Monoclinic

P $21 / \mathrm{n}$

$\mathrm{a}=13.546(4) \AA$

$\alpha=90^{\circ}$.

$\mathrm{b}=16.538(3) \AA$

$\beta=107.378(8)^{\circ}$.

$\mathrm{c}=21.279(5) \AA$

Volume

4549.4(19) $\AA^{3}$

$\gamma=90^{\circ}$.

Z

Density (calculated)

4

Absorption coefficient

$1.191 \mathrm{Mg} / \mathrm{m}^{3}$

$\mathrm{F}(000)$

Crystal size

Theta range for data collection

Index ranges

Reflections collected

Independent reflections

Completeness to theta $=24.999^{\circ}$

Absorption correction

Max. and min. transmission

$0.559 \mathrm{~mm}^{-1}$

1696

$0.32 \times 0.3 \times 0.27 \mathrm{~mm}^{3}$

1.588 to $24.999^{\circ}$.

$-16<=\mathrm{h}<=16,-19<=\mathrm{k}<=15,-25<=1<=25$

36814

$8013[\mathrm{R}(\mathrm{int})=0.0679]$

$100.0 \%$

Semi-empirical from equivalents

0.0962 and 0.0653

Refinement method

Data / restraints / parameters

Goodness-of-fit on $\mathrm{F}^{2}$

Full-matrix least-squares on $\mathrm{F}^{2}$

8013 / 0 / 451

Final $\mathrm{R}$ indices [I $>2 \operatorname{sigma}(\mathrm{I})]$

$\mathrm{R}$ indices (all data)

Extinction coefficient

Largest diff. peak and hole

1.087

$\mathrm{R} 1=0.0375, \mathrm{wR} 2=0.0867$

$\mathrm{R} 1=0.0502, \mathrm{wR} 2=0.0989$

$\mathrm{n} / \mathrm{a}$

0.610 and -0.406 e. $\AA^{-3}$ 
Table S22. Atomic coordinates ( x 10 $0^{4}$ ) and equivalent isotropic displacement parameters $\left(\AA^{2} \mathrm{x} 10^{3}\right)$ for $\mathbf{4 b}$. $U(\mathrm{eq})$ is defined as one third of the trace of the orthogonalized $\mathrm{U}^{\mathrm{ij}}$ tensor.

\begin{tabular}{|c|c|c|c|c|}
\hline & $\mathrm{x}$ & $\mathrm{y}$ & z & $\mathrm{U}(\mathrm{eq})$ \\
\hline $\mathrm{Ru}(1)$ & 1206(1) & $6870(1)$ & 6063(1) & $20(1)$ \\
\hline $\mathrm{Cl}(1)$ & $-483(1)$ & $6376(1)$ & $5516(1)$ & $26(1)$ \\
\hline $\mathrm{P}(1)$ & $905(1)$ & $8146(1)$ & $5618(1)$ & $22(1)$ \\
\hline $\mathrm{Cl}(2)$ & $2663(1)$ & $7404(1)$ & $6887(1)$ & $29(1)$ \\
\hline $\mathrm{P}(2)$ & $1884(1)$ & $6272(1)$ & $5376(1)$ & $21(1)$ \\
\hline $\mathrm{N}(1)$ & $1352(2)$ & $5706(2)$ & $6527(1)$ & $23(1)$ \\
\hline$C(7)$ & $1639(2)$ & $5204(2)$ & 5518(1) & $27(1)$ \\
\hline$C(6)$ & $1510(2)$ & $5057(2)$ & $6186(2)$ & $26(1)$ \\
\hline$C(26)$ & 1994(2) & $8837(2)$ & 5719(1) & $24(1)$ \\
\hline$C(15)$ & $4972(2)$ & $6680(2)$ & $5414(2)$ & $27(1)$ \\
\hline$C(13)$ & $3828(2)$ & $6813(2)$ & 4338(2) & $28(1)$ \\
\hline$C(5)$ & $1549(2)$ & $4277(2)$ & 6434(2) & $32(1)$ \\
\hline$C(27)$ & 2907(3) & $8539(2)$ & $5638(2)$ & $32(1)$ \\
\hline$C(31)$ & 1911(2) & $9664(2)$ & $5820(1)$ & $28(1)$ \\
\hline$C(4)$ & $1408(3)$ & $4157(2)$ & 7042(2) & $38(1)$ \\
\hline$C(12)$ & $3019(2)$ & $6563(2)$ & 4572(1) & $24(1)$ \\
\hline$C(14)$ & $4822(2)$ & $6906(2)$ & 4764(2) & $27(1)$ \\
\hline$C(1)$ & $957(3)$ & $6329(2)$ & 7463(2) & $38(1)$ \\
\hline$C(32)$ & $111(2)$ & $8626(2)$ & 6078(2) & $26(1)$ \\
\hline$C(8)$ & $1195(2)$ & $6457(2)$ & 4510(1) & $27(1)$ \\
\hline$C(23)$ & $3816(2)$ & $5632(2)$ & 6621(2) & $31(1)$ \\
\hline$C(43)$ & $545(3)$ & $8848(2)$ & 4351(1) & $29(1)$ \\
\hline$C(16)$ & $4208(2)$ & 6391(2) & 5671(1) & $24(1)$ \\
\hline$C(3)$ & $1223(3)$ & $4821(2)$ & 7384(2) & $36(1)$ \\
\hline$C(38)$ & $172(2)$ & $8334(2)$ & 4747(1) & $24(1)$ \\
\hline$C(11)$ & 1943(3) & $5514(2)$ & $3840(2)$ & $39(1)$ \\
\hline$C(42)$ & $1(3)$ & $8953(2)$ & $3690(2)$ & $35(1)$ \\
\hline$C(33)$ & $-897(3)$ & $8885(2)$ & $5789(2)$ & $39(1)$ \\
\hline$C(21)$ & $6208(3)$ & $7956(2)$ & 4949(2) & $51(1)$ \\
\hline$C(22)$ & $4629(2)$ & $6053(2)$ & $6380(1)$ & $29(1)$ \\
\hline$C(40)$ & $-1287(3)$ & $8030(2)$ & $3805(2)$ & $33(1)$ \\
\hline
\end{tabular}




\begin{tabular}{lrrrl}
$\mathrm{C}(29)$ & $3617(3)$ & $9880(2)$ & $5732(2)$ & $40(1)$ \\
$\mathrm{C}(17)$ & $3176(2)$ & $6389(2)$ & $5244(1)$ & $22(1)$ \\
$\mathrm{C}(37)$ & $523(3)$ & $8664(2)$ & $6764(2)$ & $37(1)$ \\
$\mathrm{C}(39)$ & $-753(3)$ & $7922(2)$ & $4466(2)$ & $30(1)$ \\
$\mathrm{C}(30)$ & $2717(3)$ & $10183(2)$ & $5825(2)$ & $33(1)$ \\
$\mathrm{C}(28)$ & $3713(3)$ & $9058(2)$ & $5644(2)$ & $42(1)$ \\
$\mathrm{C}(9)$ & $1944(2)$ & $6385(2)$ & $4098(1)$ & $27(1)$ \\
$\mathrm{C}(10)$ & $1615(3)$ & $6971(2)$ & $3514(2)$ & $39(1)$ \\
$\mathrm{C}(41)$ & $-910(3)$ & $8537(2)$ & $3414(2)$ & $35(1)$ \\
$\mathrm{C}(2)$ & $1181(2)$ & $5590(2)$ & $7120(1)$ & $28(1)$ \\
$\mathrm{C}(24)$ & $5129(3)$ & $6739(2)$ & $6859(2)$ & $40(1)$ \\
$\mathrm{C}(25)$ & $5453(3)$ & $5408(2)$ & $6379(2)$ & $44(1)$ \\
$\mathrm{C}(18)$ & $5746(3)$ & $7203(2)$ & $4552(2)$ & $36(1)$ \\
$\mathrm{C}(36)$ & $-75(3)$ & $8949(2)$ & $7145(2)$ & $46(1)$ \\
$\mathrm{C}(34)$ & $-1492(3)$ & $9167(3)$ & $6171(2)$ & $58(1)$ \\
$\mathrm{C}(20)$ & $6577(3)$ & $6538(2)$ & $4692(2)$ & $50(1)$ \\
$\mathrm{C}(35)$ & $-1083(3)$ & $9188(3)$ & $6852(2)$ & $57(1)$ \\
$\mathrm{C}(19)$ & $5456(3)$ & $7422(3)$ & $3828(2)$ & $69(1)$ \\
& & & & \\
\hline
\end{tabular}


Table S23. Bond lengths $[\AA]$ and angles $\left[^{\circ}\right]$ for $\mathbf{4 b}$.

\begin{tabular}{|c|c|}
\hline $\mathrm{Ru}(1)-\mathrm{Cl}(1)$ & $2.3809(9)$ \\
\hline $\mathrm{Ru}(1)-\mathrm{P}(1)$ & $2.2986(9)$ \\
\hline $\mathrm{Ru}(1)-\mathrm{Cl}(2)$ & $2.3834(9)$ \\
\hline $\mathrm{Ru}(1)-\mathrm{P}(2)$ & $2.1826(9)$ \\
\hline $\mathrm{Ru}(1)-\mathrm{N}(1)$ & $2.145(2)$ \\
\hline $\mathrm{P}(1)-\mathrm{C}(26)$ & $1.829(3)$ \\
\hline $\mathrm{P}(1)-\mathrm{C}(32)$ & $1.837(3)$ \\
\hline $\mathrm{P}(1)-\mathrm{C}(38)$ & $1.849(3)$ \\
\hline$P(2)-C(7)$ & $1.838(3)$ \\
\hline $\mathrm{P}(2)-\mathrm{C}(8)$ & $1.826(3)$ \\
\hline $\mathrm{P}(2)-\mathrm{C}(17)$ & $1.863(3)$ \\
\hline $\mathrm{N}(1)-\mathrm{C}(6)$ & $1.349(4)$ \\
\hline $\mathrm{N}(1)-\mathrm{C}(2)$ & $1.362(4)$ \\
\hline $\mathrm{C}(7)-\mathrm{H}(7 \mathrm{~A})$ & 0.9900 \\
\hline $\mathrm{C}(7)-\mathrm{H}(7 \mathrm{~B})$ & 0.9900 \\
\hline$C(7)-C(6)$ & $1.504(4)$ \\
\hline $\mathrm{C}(6)-\mathrm{C}(5)$ & $1.389(4)$ \\
\hline$C(26)-C(27)$ & $1.389(5)$ \\
\hline$C(26)-C(31)$ & $1.395(4)$ \\
\hline $\mathrm{C}(15)-\mathrm{H}(15)$ & 0.9500 \\
\hline$C(15)-C(14)$ & $1.387(4)$ \\
\hline$C(15)-C(16)$ & $1.391(4)$ \\
\hline $\mathrm{C}(13)-\mathrm{H}(13)$ & 0.9500 \\
\hline $\mathrm{C}(13)-\mathrm{C}(12)$ & $1.395(4)$ \\
\hline$C(13)-C(14)$ & $1.389(4)$ \\
\hline $\mathrm{C}(5)-\mathrm{H}(5)$ & 0.9500 \\
\hline$C(5)-C(4)$ & $1.376(5)$ \\
\hline $\mathrm{C}(27)-\mathrm{H}(27)$ & 0.9500 \\
\hline$C(27)-C(28)$ & $1.386(5)$ \\
\hline $\mathrm{C}(31)-\mathrm{H}(31)$ & 0.9500 \\
\hline$C(31)-C(30)$ & $1.386(5)$ \\
\hline $\mathrm{C}(4)-\mathrm{H}(4)$ & 0.9500 \\
\hline $\mathrm{C}(4)-\mathrm{C}(3)$ & $1.380(5)$ \\
\hline$C(12)-C(17)$ & $1.411(4)$ \\
\hline
\end{tabular}




\begin{tabular}{|c|c|}
\hline $\mathrm{C}(12)-\mathrm{C}(9)$ & $1.532(4)$ \\
\hline$C(14)-C(18)$ & $1.533(4)$ \\
\hline C(1)-H(1A) & 0.9800 \\
\hline $\mathrm{C}(1)-\mathrm{H}(1 \mathrm{~B})$ & 0.9800 \\
\hline C(1)-H(1C) & 0.9800 \\
\hline$C(1)-C(2)$ & $1.500(5)$ \\
\hline C(32)-C(33) & $1.388(5)$ \\
\hline C(32)-C(37) & $1.398(4)$ \\
\hline $\mathrm{C}(8)-\mathrm{H}(8 \mathrm{~A})$ & 0.9900 \\
\hline C(8)-H(8B) & 0.9900 \\
\hline $\mathrm{C}(8)-\mathrm{C}(9)$ & $1.530(4)$ \\
\hline $\mathrm{C}(23)-\mathrm{H}(23 \mathrm{~A})$ & 0.9800 \\
\hline $\mathrm{C}(23)-\mathrm{H}(23 \mathrm{~B})$ & 0.9800 \\
\hline $\mathrm{C}(23)-\mathrm{H}(23 \mathrm{C})$ & 0.9800 \\
\hline $\mathrm{C}(23)-\mathrm{C}(22)$ & $1.516(4)$ \\
\hline C(43)-H(43) & 0.9500 \\
\hline C(43)-C(38) & $1.393(4)$ \\
\hline C(43)-C(42) & $1.390(4)$ \\
\hline$C(16)-C(22)$ & $1.549(4)$ \\
\hline C(16)-C(17) & $1.422(4)$ \\
\hline $\mathrm{C}(3)-\mathrm{H}(3)$ & 0.9500 \\
\hline$C(3)-C(2)$ & $1.385(5)$ \\
\hline C(38)-C(39) & $1.394(4)$ \\
\hline $\mathrm{C}(11)-\mathrm{H}(11 \mathrm{~A})$ & 0.9800 \\
\hline $\mathrm{C}(11)-\mathrm{H}(11 \mathrm{~B})$ & 0.9800 \\
\hline C(11)-H(11C) & 0.9800 \\
\hline C(11)-C(9) & $1.542(5)$ \\
\hline $\mathrm{C}(42)-\mathrm{H}(42)$ & 0.9500 \\
\hline $\mathrm{C}(42)-\mathrm{C}(41)$ & $1.381(5)$ \\
\hline C(33)-H(33) & 0.9500 \\
\hline C(33)-C(34) & $1.387(5)$ \\
\hline $\mathrm{C}(21)-\mathrm{H}(21 \mathrm{~A})$ & 0.9800 \\
\hline $\mathrm{C}(21)-\mathrm{H}(21 \mathrm{~B})$ & 0.9800 \\
\hline $\mathrm{C}(21)-\mathrm{H}(21 \mathrm{C})$ & 0.9800 \\
\hline $\mathrm{C}(21)-\mathrm{C}(18)$ & $1.529(5)$ \\
\hline C(22)-C(24) & $1.540(5)$ \\
\hline
\end{tabular}




\begin{tabular}{|c|c|}
\hline$C(22)-C(25)$ & $1.544(5)$ \\
\hline $\mathrm{C}(40)-\mathrm{H}(40)$ & 0.9500 \\
\hline C(40)-C(39) & $1.388(4)$ \\
\hline $\mathrm{C}(40)-\mathrm{C}(41)$ & $1.381(5)$ \\
\hline C(29)-H(29) & 0.9500 \\
\hline C(29)-C(30) & $1.386(5)$ \\
\hline C(29)-C(28) & $1.385(5)$ \\
\hline C(37)-H(37) & 0.9500 \\
\hline C(37)-C(36) & $1.389(5)$ \\
\hline C(39)-H(39) & 0.9500 \\
\hline $\mathrm{C}(30)-\mathrm{H}(30)$ & 0.9500 \\
\hline C(28)-H(28) & 0.9500 \\
\hline$C(9)-C(10)$ & $1.533(4)$ \\
\hline $\mathrm{C}(10)-\mathrm{H}(10 \mathrm{~A})$ & 0.9800 \\
\hline C(10)-H(10B) & 0.9800 \\
\hline $\mathrm{C}(10)-\mathrm{H}(10 \mathrm{C})$ & 0.9800 \\
\hline $\mathrm{C}(41)-\mathrm{H}(41)$ & 0.9500 \\
\hline $\mathrm{C}(24)-\mathrm{H}(24 \mathrm{~A})$ & 0.9800 \\
\hline C(24)-H(24B) & 0.9800 \\
\hline $\mathrm{C}(24)-\mathrm{H}(24 \mathrm{C})$ & 0.9800 \\
\hline $\mathrm{C}(25)-\mathrm{H}(25 \mathrm{~A})$ & 0.9800 \\
\hline $\mathrm{C}(25)-\mathrm{H}(25 \mathrm{~B})$ & 0.9800 \\
\hline $\mathrm{C}(25)-\mathrm{H}(25 \mathrm{C})$ & 0.9800 \\
\hline$C(18)-C(20)$ & $1.538(5)$ \\
\hline C(18)-C(19) & $1.516(5)$ \\
\hline C(36)-H(36) & 0.9500 \\
\hline$C(36)-C(35)$ & $1.379(6)$ \\
\hline C(34)-H(34) & 0.9500 \\
\hline C(34)-C(35) & $1.387(6)$ \\
\hline $\mathrm{C}(20)-\mathrm{H}(20 \mathrm{~A})$ & 0.9800 \\
\hline $\mathrm{C}(20)-\mathrm{H}(20 \mathrm{~B})$ & 0.9800 \\
\hline $\mathrm{C}(20)-\mathrm{H}(20 \mathrm{C})$ & 0.9800 \\
\hline C(35)-H(35) & 0.9500 \\
\hline C(19)-H(19A) & 0.9800 \\
\hline C(19)-H(19B) & 0.9800 \\
\hline C(19)-H(19C) & 0.9800 \\
\hline
\end{tabular}




\begin{tabular}{|c|c|}
\hline $\mathrm{Cl}(1)-\mathrm{Ru}(1)-\mathrm{Cl}(2)$ & $161.88(3)$ \\
\hline $\mathrm{P}(1)-\mathrm{Ru}(1)-\mathrm{Cl}(1)$ & $94.98(3)$ \\
\hline $\mathrm{P}(1)-\mathrm{Ru}(1)-\mathrm{Cl}(2)$ & $88.25(3)$ \\
\hline $\mathrm{P}(2)-\mathrm{Ru}(1)-\mathrm{Cl}(1)$ & $93.01(3)$ \\
\hline $\mathrm{P}(2)-\mathrm{Ru}(1)-\mathrm{P}(1)$ & $101.44(3)$ \\
\hline $\mathrm{P}(2)-\mathrm{Ru}(1)-\mathrm{Cl}(2)$ & $103.84(3)$ \\
\hline $\mathrm{N}(1)-\mathrm{Ru}(1)-\mathrm{Cl}(1)$ & $81.94(7)$ \\
\hline $\mathrm{N}(1)-\mathrm{Ru}(1)-\mathrm{P}(1)$ & $173.55(7)$ \\
\hline $\mathrm{N}(1)-\mathrm{Ru}(1)-\mathrm{Cl}(2)$ & $92.97(7)$ \\
\hline $\mathrm{N}(1)-\mathrm{Ru}(1)-\mathrm{P}(2)$ & $84.43(7)$ \\
\hline$C(26)-P(1)-R u(1)$ & $119.19(10)$ \\
\hline C(26)-P(1)-C(32) & $104.28(14)$ \\
\hline C(26)-P(1)-C(38) & $100.66(14)$ \\
\hline $\mathrm{C}(32)-\mathrm{P}(1)-\mathrm{Ru}(1)$ & $103.73(10)$ \\
\hline C(32)-P(1)-C(38) & $103.73(14)$ \\
\hline C(38)-P(1)-Ru(1) & $122.98(10)$ \\
\hline$C(7)-P(2)-R u(1)$ & $101.08(10)$ \\
\hline $\mathrm{C}(7)-\mathrm{P}(2)-\mathrm{C}(17)$ & $110.42(14)$ \\
\hline $\mathrm{C}(8)-\mathrm{P}(2)-\mathrm{Ru}(1)$ & $114.16(10)$ \\
\hline $\mathrm{C}(8)-\mathrm{P}(2)-\mathrm{C}(7)$ & $105.29(14)$ \\
\hline C(8)-P(2)-C(17) & $92.94(14)$ \\
\hline $\mathrm{C}(17)-\mathrm{P}(2)-\mathrm{Ru}(1)$ & $130.98(9)$ \\
\hline $\mathrm{C}(6)-\mathrm{N}(1)-\mathrm{Ru}(1)$ & $118.30(19)$ \\
\hline $\mathrm{C}(6)-\mathrm{N}(1)-\mathrm{C}(2)$ & $119.0(3)$ \\
\hline $\mathrm{C}(2)-\mathrm{N}(1)-\mathrm{Ru}(1)$ & $122.4(2)$ \\
\hline $\mathrm{P}(2)-\mathrm{C}(7)-\mathrm{H}(7 \mathrm{~A})$ & 109.0 \\
\hline $\mathrm{P}(2)-\mathrm{C}(7)-\mathrm{H}(7 \mathrm{~B})$ & 109.0 \\
\hline $\mathrm{H}(7 \mathrm{~A})-\mathrm{C}(7)-\mathrm{H}(7 \mathrm{~B})$ & 107.8 \\
\hline $\mathrm{C}(6)-\mathrm{C}(7)-\mathrm{P}(2)$ & $112.9(2)$ \\
\hline $\mathrm{C}(6)-\mathrm{C}(7)-\mathrm{H}(7 \mathrm{~A})$ & 109.0 \\
\hline $\mathrm{C}(6)-\mathrm{C}(7)-\mathrm{H}(7 \mathrm{~B})$ & 109.0 \\
\hline $\mathrm{N}(1)-\mathrm{C}(6)-\mathrm{C}(7)$ & $117.5(3)$ \\
\hline $\mathrm{N}(1)-\mathrm{C}(6)-\mathrm{C}(5)$ & $122.0(3)$ \\
\hline$C(5)-C(6)-C(7)$ & $120.5(3)$ \\
\hline $\mathrm{C}(27)-\mathrm{C}(26)-\mathrm{P}(1)$ & $118.7(2)$ \\
\hline
\end{tabular}




\begin{tabular}{|c|c|}
\hline $\mathrm{C}(27)-\mathrm{C}(26)-\mathrm{C}(31)$ & $118.9(3)$ \\
\hline $\mathrm{C}(31)-\mathrm{C}(26)-\mathrm{P}(1)$ & $122.1(2)$ \\
\hline $\mathrm{C}(14)-\mathrm{C}(15)-\mathrm{H}(15)$ & 117.3 \\
\hline$C(14)-C(15)-C(16)$ & $125.4(3)$ \\
\hline $\mathrm{C}(16)-\mathrm{C}(15)-\mathrm{H}(15)$ & 117.3 \\
\hline $\mathrm{C}(12)-\mathrm{C}(13)-\mathrm{H}(13)$ & 119.6 \\
\hline $\mathrm{C}(14)-\mathrm{C}(13)-\mathrm{H}(13)$ & 119.6 \\
\hline $\mathrm{C}(14)-\mathrm{C}(13)-\mathrm{C}(12)$ & $120.8(3)$ \\
\hline $\mathrm{C}(6)-\mathrm{C}(5)-\mathrm{H}(5)$ & 120.3 \\
\hline$C(4)-C(5)-C(6)$ & $119.3(3)$ \\
\hline $\mathrm{C}(4)-\mathrm{C}(5)-\mathrm{H}(5)$ & 120.3 \\
\hline $\mathrm{C}(26)-\mathrm{C}(27)-\mathrm{H}(27)$ & 119.7 \\
\hline $\mathrm{C}(28)-\mathrm{C}(27)-\mathrm{C}(26)$ & $120.5(3)$ \\
\hline $\mathrm{C}(28)-\mathrm{C}(27)-\mathrm{H}(27)$ & 119.7 \\
\hline $\mathrm{C}(26)-\mathrm{C}(31)-\mathrm{H}(31)$ & 119.8 \\
\hline $\mathrm{C}(30)-\mathrm{C}(31)-\mathrm{C}(26)$ & $120.5(3)$ \\
\hline $\mathrm{C}(30)-\mathrm{C}(31)-\mathrm{H}(31)$ & 119.8 \\
\hline $\mathrm{C}(5)-\mathrm{C}(4)-\mathrm{H}(4)$ & 120.7 \\
\hline $\mathrm{C}(5)-\mathrm{C}(4)-\mathrm{C}(3)$ & $118.6(3)$ \\
\hline $\mathrm{C}(3)-\mathrm{C}(4)-\mathrm{H}(4)$ & 120.7 \\
\hline $\mathrm{C}(13)-\mathrm{C}(12)-\mathrm{C}(17)$ & $121.7(3)$ \\
\hline$C(13)-C(12)-C(9)$ & 121.1(3) \\
\hline$C(17)-C(12)-C(9)$ & $117.0(3)$ \\
\hline$C(15)-C(14)-C(13)$ & $116.5(3)$ \\
\hline $\mathrm{C}(15)-\mathrm{C}(14)-\mathrm{C}(18)$ & $119.3(3)$ \\
\hline $\mathrm{C}(13)-\mathrm{C}(14)-\mathrm{C}(18)$ & $124.1(3)$ \\
\hline $\mathrm{H}(1 \mathrm{~A})-\mathrm{C}(1)-\mathrm{H}(1 \mathrm{~B})$ & 109.5 \\
\hline $\mathrm{H}(1 \mathrm{~A})-\mathrm{C}(1)-\mathrm{H}(1 \mathrm{C})$ & 109.5 \\
\hline $\mathrm{H}(1 \mathrm{~B})-\mathrm{C}(1)-\mathrm{H}(1 \mathrm{C})$ & 109.5 \\
\hline $\mathrm{C}(2)-\mathrm{C}(1)-\mathrm{H}(1 \mathrm{~A})$ & 109.5 \\
\hline $\mathrm{C}(2)-\mathrm{C}(1)-\mathrm{H}(1 \mathrm{~B})$ & 109.5 \\
\hline $\mathrm{C}(2)-\mathrm{C}(1)-\mathrm{H}(1 \mathrm{C})$ & 109.5 \\
\hline C(33)-C(32)-P(1) & $123.4(2)$ \\
\hline $\mathrm{C}(33)-\mathrm{C}(32)-\mathrm{C}(37)$ & $118.8(3)$ \\
\hline $\mathrm{C}(37)-\mathrm{C}(32)-\mathrm{P}(1)$ & $117.5(2)$ \\
\hline $\mathrm{P}(2)-\mathrm{C}(8)-\mathrm{H}(8 \mathrm{~A})$ & 109.7 \\
\hline
\end{tabular}




\begin{tabular}{|c|c|}
\hline $\mathrm{P}(2)-\mathrm{C}(8)-\mathrm{H}(8 \mathrm{~B})$ & 109.7 \\
\hline $\mathrm{H}(8 \mathrm{~A})-\mathrm{C}(8)-\mathrm{H}(8 \mathrm{~B})$ & 108.2 \\
\hline $\mathrm{C}(9)-\mathrm{C}(8)-\mathrm{P}(2)$ & $109.7(2)$ \\
\hline $\mathrm{C}(9)-\mathrm{C}(8)-\mathrm{H}(8 \mathrm{~A})$ & 109.7 \\
\hline $\mathrm{C}(9)-\mathrm{C}(8)-\mathrm{H}(8 \mathrm{~B})$ & 109.7 \\
\hline $\mathrm{H}(23 \mathrm{~A})-\mathrm{C}(23)-\mathrm{H}(23 \mathrm{~B})$ & 109.5 \\
\hline $\mathrm{H}(23 \mathrm{~A})-\mathrm{C}(23)-\mathrm{H}(23 \mathrm{C})$ & 109.5 \\
\hline $\mathrm{H}(23 \mathrm{~B})-\mathrm{C}(23)-\mathrm{H}(23 \mathrm{C})$ & 109.5 \\
\hline $\mathrm{C}(22)-\mathrm{C}(23)-\mathrm{H}(23 \mathrm{~A})$ & 109.5 \\
\hline $\mathrm{C}(22)-\mathrm{C}(23)-\mathrm{H}(23 \mathrm{~B})$ & 109.5 \\
\hline $\mathrm{C}(22)-\mathrm{C}(23)-\mathrm{H}(23 \mathrm{C})$ & 109.5 \\
\hline $\mathrm{C}(38)-\mathrm{C}(43)-\mathrm{H}(43)$ & 119.7 \\
\hline $\mathrm{C}(42)-\mathrm{C}(43)-\mathrm{H}(43)$ & 119.7 \\
\hline$C(42)-C(43)-C(38)$ & $120.6(3)$ \\
\hline $\mathrm{C}(15)-\mathrm{C}(16)-\mathrm{C}(22)$ & $114.0(3)$ \\
\hline$C(15)-C(16)-C(17)$ & $117.0(3)$ \\
\hline $\mathrm{C}(17)-\mathrm{C}(16)-\mathrm{C}(22)$ & $128.8(3)$ \\
\hline $\mathrm{C}(4)-\mathrm{C}(3)-\mathrm{H}(3)$ & 119.7 \\
\hline$C(4)-C(3)-C(2)$ & $120.6(3)$ \\
\hline $\mathrm{C}(2)-\mathrm{C}(3)-\mathrm{H}(3)$ & 119.7 \\
\hline $\mathrm{C}(43)-\mathrm{C}(38)-\mathrm{P}(1)$ & $121.7(2)$ \\
\hline $\mathrm{C}(43)-\mathrm{C}(38)-\mathrm{C}(39)$ & $118.8(3)$ \\
\hline $\mathrm{C}(39)-\mathrm{C}(38)-\mathrm{P}(1)$ & $119.4(2)$ \\
\hline $\mathrm{H}(11 \mathrm{~A})-\mathrm{C}(11)-\mathrm{H}(11 \mathrm{~B})$ & 109.5 \\
\hline $\mathrm{H}(11 \mathrm{~A})-\mathrm{C}(11)-\mathrm{H}(11 \mathrm{C})$ & 109.5 \\
\hline $\mathrm{H}(11 \mathrm{~B})-\mathrm{C}(11)-\mathrm{H}(11 \mathrm{C})$ & 109.5 \\
\hline $\mathrm{C}(9)-\mathrm{C}(11)-\mathrm{H}(11 \mathrm{~A})$ & 109.5 \\
\hline $\mathrm{C}(9)-\mathrm{C}(11)-\mathrm{H}(11 \mathrm{~B})$ & 109.5 \\
\hline $\mathrm{C}(9)-\mathrm{C}(11)-\mathrm{H}(11 \mathrm{C})$ & 109.5 \\
\hline $\mathrm{C}(43)-\mathrm{C}(42)-\mathrm{H}(42)$ & 119.9 \\
\hline $\mathrm{C}(41)-\mathrm{C}(42)-\mathrm{C}(43)$ & $120.2(3)$ \\
\hline $\mathrm{C}(41)-\mathrm{C}(42)-\mathrm{H}(42)$ & 119.9 \\
\hline $\mathrm{C}(32)-\mathrm{C}(33)-\mathrm{H}(33)$ & 119.6 \\
\hline $\mathrm{C}(34)-\mathrm{C}(33)-\mathrm{C}(32)$ & $120.7(3)$ \\
\hline $\mathrm{C}(34)-\mathrm{C}(33)-\mathrm{H}(33)$ & 119.6 \\
\hline $\mathrm{H}(21 \mathrm{~A})-\mathrm{C}(21)-\mathrm{H}(21 \mathrm{~B})$ & 109.5 \\
\hline
\end{tabular}




\begin{tabular}{|c|c|}
\hline $\mathrm{H}(21 \mathrm{~A})-\mathrm{C}(21)-\mathrm{H}(21 \mathrm{C})$ & 109.5 \\
\hline $\mathrm{H}(21 \mathrm{~B})-\mathrm{C}(21)-\mathrm{H}(21 \mathrm{C})$ & 109.5 \\
\hline $\mathrm{C}(18)-\mathrm{C}(21)-\mathrm{H}(21 \mathrm{~A})$ & 109.5 \\
\hline $\mathrm{C}(18)-\mathrm{C}(21)-\mathrm{H}(21 \mathrm{~B})$ & 109.5 \\
\hline $\mathrm{C}(18)-\mathrm{C}(21)-\mathrm{H}(21 \mathrm{C})$ & 109.5 \\
\hline$C(23)-C(22)-C(16)$ & $113.7(3)$ \\
\hline$C(23)-C(22)-C(24)$ & $109.6(3)$ \\
\hline$C(23)-C(22)-C(25)$ & $106.4(3)$ \\
\hline$C(24)-C(22)-C(16)$ & $109.8(3)$ \\
\hline $\mathrm{C}(24)-\mathrm{C}(22)-\mathrm{C}(25)$ & $109.5(3)$ \\
\hline$C(25)-C(22)-C(16)$ & $107.7(3)$ \\
\hline $\mathrm{C}(39)-\mathrm{C}(40)-\mathrm{H}(40)$ & 119.5 \\
\hline $\mathrm{C}(41)-\mathrm{C}(40)-\mathrm{H}(40)$ & 119.5 \\
\hline$C(41)-C(40)-C(39)$ & $120.9(3)$ \\
\hline $\mathrm{C}(30)-\mathrm{C}(29)-\mathrm{H}(29)$ & 120.1 \\
\hline $\mathrm{C}(28)-\mathrm{C}(29)-\mathrm{H}(29)$ & 120.1 \\
\hline$C(28)-C(29)-C(30)$ & $119.8(3)$ \\
\hline $\mathrm{C}(12)-\mathrm{C}(17)-\mathrm{P}(2)$ & $108.0(2)$ \\
\hline$C(12)-C(17)-C(16)$ & $118.0(3)$ \\
\hline $\mathrm{C}(16)-\mathrm{C}(17)-\mathrm{P}(2)$ & $133.8(2)$ \\
\hline $\mathrm{C}(32)-\mathrm{C}(37)-\mathrm{H}(37)$ & 119.8 \\
\hline$C(36)-C(37)-C(32)$ & $120.3(3)$ \\
\hline $\mathrm{C}(36)-\mathrm{C}(37)-\mathrm{H}(37)$ & 119.8 \\
\hline $\mathrm{C}(38)-\mathrm{C}(39)-\mathrm{H}(39)$ & 120.0 \\
\hline $\mathrm{C}(40)-\mathrm{C}(39)-\mathrm{C}(38)$ & $120.0(3)$ \\
\hline $\mathrm{C}(40)-\mathrm{C}(39)-\mathrm{H}(39)$ & 120.0 \\
\hline $\mathrm{C}(31)-\mathrm{C}(30)-\mathrm{H}(30)$ & 120.0 \\
\hline$C(29)-C(30)-C(31)$ & $120.0(3)$ \\
\hline $\mathrm{C}(29)-\mathrm{C}(30)-\mathrm{H}(30)$ & 120.0 \\
\hline $\mathrm{C}(27)-\mathrm{C}(28)-\mathrm{H}(28)$ & 119.9 \\
\hline$C(29)-C(28)-C(27)$ & $120.2(3)$ \\
\hline $\mathrm{C}(29)-\mathrm{C}(28)-\mathrm{H}(28)$ & 119.9 \\
\hline$C(12)-C(9)-C(11)$ & $108.3(2)$ \\
\hline$C(12)-C(9)-C(10)$ & 113.6(3) \\
\hline $\mathrm{C}(8)-\mathrm{C}(9)-\mathrm{C}(12)$ & $105.7(2)$ \\
\hline $\mathrm{C}(8)-\mathrm{C}(9)-\mathrm{C}(11)$ & $110.3(3)$ \\
\hline
\end{tabular}




\begin{tabular}{|c|c|}
\hline $\mathrm{C}(8)-\mathrm{C}(9)-\mathrm{C}(10)$ & $109.4(3)$ \\
\hline$C(10)-C(9)-C(11)$ & $109.4(3)$ \\
\hline $\mathrm{C}(9)-\mathrm{C}(10)-\mathrm{H}(10 \mathrm{~A})$ & 109.5 \\
\hline $\mathrm{C}(9)-\mathrm{C}(10)-\mathrm{H}(10 \mathrm{~B})$ & 109.5 \\
\hline $\mathrm{C}(9)-\mathrm{C}(10)-\mathrm{H}(10 \mathrm{C})$ & 109.5 \\
\hline $\mathrm{H}(10 \mathrm{~A})-\mathrm{C}(10)-\mathrm{H}(10 \mathrm{~B})$ & 109.5 \\
\hline $\mathrm{H}(10 \mathrm{~A})-\mathrm{C}(10)-\mathrm{H}(10 \mathrm{C})$ & 109.5 \\
\hline $\mathrm{H}(10 \mathrm{~B})-\mathrm{C}(10)-\mathrm{H}(10 \mathrm{C})$ & 109.5 \\
\hline $\mathrm{C}(42)-\mathrm{C}(41)-\mathrm{C}(40)$ & $119.4(3)$ \\
\hline $\mathrm{C}(42)-\mathrm{C}(41)-\mathrm{H}(41)$ & 120.3 \\
\hline $\mathrm{C}(40)-\mathrm{C}(41)-\mathrm{H}(41)$ & 120.3 \\
\hline $\mathrm{N}(1)-\mathrm{C}(2)-\mathrm{C}(1)$ & $116.8(3)$ \\
\hline $\mathrm{N}(1)-\mathrm{C}(2)-\mathrm{C}(3)$ & $120.4(3)$ \\
\hline$C(3)-C(2)-C(1)$ & $122.7(3)$ \\
\hline $\mathrm{C}(22)-\mathrm{C}(24)-\mathrm{H}(24 \mathrm{~A})$ & 109.5 \\
\hline $\mathrm{C}(22)-\mathrm{C}(24)-\mathrm{H}(24 \mathrm{~B})$ & 109.5 \\
\hline $\mathrm{C}(22)-\mathrm{C}(24)-\mathrm{H}(24 \mathrm{C})$ & 109.5 \\
\hline $\mathrm{H}(24 \mathrm{~A})-\mathrm{C}(24)-\mathrm{H}(24 \mathrm{~B})$ & 109.5 \\
\hline $\mathrm{H}(24 \mathrm{~A})-\mathrm{C}(24)-\mathrm{H}(24 \mathrm{C})$ & 109.5 \\
\hline $\mathrm{H}(24 \mathrm{~B})-\mathrm{C}(24)-\mathrm{H}(24 \mathrm{C})$ & 109.5 \\
\hline $\mathrm{C}(22)-\mathrm{C}(25)-\mathrm{H}(25 \mathrm{~A})$ & 109.5 \\
\hline $\mathrm{C}(22)-\mathrm{C}(25)-\mathrm{H}(25 \mathrm{~B})$ & 109.5 \\
\hline $\mathrm{C}(22)-\mathrm{C}(25)-\mathrm{H}(25 \mathrm{C})$ & 109.5 \\
\hline $\mathrm{H}(25 \mathrm{~A})-\mathrm{C}(25)-\mathrm{H}(25 \mathrm{~B})$ & 109.5 \\
\hline $\mathrm{H}(25 \mathrm{~A})-\mathrm{C}(25)-\mathrm{H}(25 \mathrm{C})$ & 109.5 \\
\hline $\mathrm{H}(25 \mathrm{~B})-\mathrm{C}(25)-\mathrm{H}(25 \mathrm{C})$ & 109.5 \\
\hline $\mathrm{C}(14)-\mathrm{C}(18)-\mathrm{C}(20)$ & $109.2(3)$ \\
\hline $\mathrm{C}(21)-\mathrm{C}(18)-\mathrm{C}(14)$ & $109.5(3)$ \\
\hline$C(21)-C(18)-C(20)$ & $108.7(3)$ \\
\hline $\mathrm{C}(19)-\mathrm{C}(18)-\mathrm{C}(14)$ & $112.9(3)$ \\
\hline $\mathrm{C}(19)-\mathrm{C}(18)-\mathrm{C}(21)$ & $107.8(3)$ \\
\hline $\mathrm{C}(19)-\mathrm{C}(18)-\mathrm{C}(20)$ & $108.6(3)$ \\
\hline $\mathrm{C}(37)-\mathrm{C}(36)-\mathrm{H}(36)$ & 119.9 \\
\hline $\mathrm{C}(35)-\mathrm{C}(36)-\mathrm{C}(37)$ & $120.2(3)$ \\
\hline $\mathrm{C}(35)-\mathrm{C}(36)-\mathrm{H}(36)$ & 119.9 \\
\hline $\mathrm{C}(33)-\mathrm{C}(34)-\mathrm{H}(34)$ & 120.0 \\
\hline
\end{tabular}




\begin{tabular}{ll}
$\mathrm{C}(35)-\mathrm{C}(34)-\mathrm{C}(33)$ & $119.9(4)$ \\
$\mathrm{C}(35)-\mathrm{C}(34)-\mathrm{H}(34)$ & 120.0 \\
$\mathrm{C}(18)-\mathrm{C}(20)-\mathrm{H}(20 \mathrm{~A})$ & 109.5 \\
$\mathrm{C}(18)-\mathrm{C}(20)-\mathrm{H}(20 \mathrm{~B})$ & 109.5 \\
$\mathrm{C}(18)-\mathrm{C}(20)-\mathrm{H}(20 \mathrm{C})$ & 109.5 \\
$\mathrm{H}(20 \mathrm{~A})-\mathrm{C}(20)-\mathrm{H}(20 \mathrm{~B})$ & 109.5 \\
$\mathrm{H}(20 \mathrm{~A})-\mathrm{C}(20)-\mathrm{H}(20 \mathrm{C})$ & 109.5 \\
$\mathrm{H}(20 \mathrm{~B})-\mathrm{C}(20)-\mathrm{H}(20 \mathrm{C})$ & 109.5 \\
$\mathrm{C}(36)-\mathrm{C}(35)-\mathrm{C}(34)$ & $120.0(4)$ \\
$\mathrm{C}(36)-\mathrm{C}(35)-\mathrm{H}(35)$ & 120.0 \\
$\mathrm{C}(34)-\mathrm{C}(35)-\mathrm{H}(35)$ & 120.0 \\
$\mathrm{C}(18)-\mathrm{C}(19)-\mathrm{H}(19 \mathrm{~A})$ & 109.5 \\
$\mathrm{C}(18)-\mathrm{C}(19)-\mathrm{H}(19 B)$ & 109.5 \\
$\mathrm{C}(18)-\mathrm{C}(19)-\mathrm{H}(19 \mathrm{C})$ & 109.5 \\
$\mathrm{H}(19 \mathrm{~A})-\mathrm{C}(19)-\mathrm{H}(19 B)$ & 109.5 \\
$\mathrm{H}(19 \mathrm{~A})-\mathrm{C}(19)-\mathrm{H}(19 \mathrm{C})$ & 109.5 \\
$\mathrm{H}(19 \mathrm{~B})-\mathrm{C}(19)-\mathrm{H}(19 \mathrm{C})$ & 109.5 \\
\hline
\end{tabular}

Symmetry transformations used to generate equivalent atoms: 
Table S24. Anisotropic displacement parameters $\left(\AA^{2} \times 10^{3}\right)$ for $\mathbf{4 b}$. The anisotropic displacement factor exponent takes the form: $-2 \pi^{2}\left[h^{2} a^{* 2} U^{11}+\ldots+2 h \mathrm{k}^{*} b^{*} U^{12}\right]$

\begin{tabular}{|c|c|c|c|c|c|c|}
\hline & $\mathrm{U}^{11}$ & $\mathrm{U}^{22}$ & $\mathrm{U}^{33}$ & $\mathrm{U}^{23}$ & $\mathrm{U}^{13}$ & $\mathrm{U}^{12}$ \\
\hline $\mathrm{Ru}(1)$ & $25(1)$ & $18(1)$ & $16(1)$ & $-1(1)$ & $4(1)$ & $0(1)$ \\
\hline $\mathrm{Cl}(1)$ & $25(1)$ & $25(1)$ & $25(1)$ & $-1(1)$ & $4(1)$ & $-2(1)$ \\
\hline $\mathrm{P}(1)$ & $27(1)$ & $19(1)$ & $18(1)$ & $-2(1)$ & $4(1)$ & $2(1)$ \\
\hline $\mathrm{Cl}(2)$ & $31(1)$ & $25(1)$ & $25(1)$ & $-2(1)$ & $-2(1)$ & $-1(1)$ \\
\hline $\mathrm{P}(2)$ & $24(1)$ & $20(1)$ & $19(1)$ & $-1(1)$ & $5(1)$ & $1(1)$ \\
\hline $\mathrm{N}(1)$ & $24(1)$ & $22(1)$ & $22(1)$ & $2(1)$ & $4(1)$ & $-2(1)$ \\
\hline$C(7)$ & $29(2)$ & $23(2)$ & $27(2)$ & $-4(1)$ & $8(1)$ & $-2(1)$ \\
\hline$C(6)$ & $22(2)$ & $21(2)$ & $32(2)$ & $0(1)$ & $5(1)$ & $-2(1)$ \\
\hline$C(26)$ & $32(2)$ & $20(2)$ & $18(1)$ & $0(1)$ & $2(1)$ & $1(1)$ \\
\hline$C(15)$ & $26(2)$ & $24(2)$ & $29(2)$ & $-4(1)$ & $6(1)$ & $3(1)$ \\
\hline$C(13)$ & $36(2)$ & $31(2)$ & $22(2)$ & $-1(1)$ & $14(1)$ & $6(1)$ \\
\hline$C(5)$ & $31(2)$ & $20(2)$ & $44(2)$ & $5(1)$ & $7(2)$ & $0(1)$ \\
\hline$C(27)$ & $36(2)$ & $24(2)$ & $38(2)$ & $1(1)$ & $12(2)$ & $3(1)$ \\
\hline $\mathrm{C}(31)$ & $32(2)$ & $22(2)$ & $25(2)$ & $1(1)$ & $4(1)$ & $4(1)$ \\
\hline$C(4)$ & $34(2)$ & $29(2)$ & $47(2)$ & $18(2)$ & $6(2)$ & $1(2)$ \\
\hline$C(12)$ & $29(2)$ & $19(2)$ & $25(2)$ & $-5(1)$ & $8(1)$ & $2(1)$ \\
\hline$C(14)$ & $30(2)$ & $25(2)$ & $29(2)$ & $-4(1)$ & $13(1)$ & $3(1)$ \\
\hline$C(1)$ & $44(2)$ & $43(2)$ & $30(2)$ & $-1(2)$ & $17(2)$ & $-3(2)$ \\
\hline$C(32)$ & $30(2)$ & $19(2)$ & $30(2)$ & $-3(1)$ & $10(1)$ & $2(1)$ \\
\hline$C(8)$ & $26(2)$ & $32(2)$ & $21(1)$ & $-3(1)$ & $6(1)$ & $6(1)$ \\
\hline$C(23)$ & $32(2)$ & $32(2)$ & $26(2)$ & $6(1)$ & $6(1)$ & $6(1)$ \\
\hline$C(43)$ & $36(2)$ & $30(2)$ & $23(2)$ & $-1(1)$ & $10(1)$ & $4(1)$ \\
\hline$C(16)$ & $27(2)$ & $20(2)$ & $24(2)$ & $-2(1)$ & $8(1)$ & $4(1)$ \\
\hline$C(3)$ & $33(2)$ & $44(2)$ & $28(2)$ & $12(2)$ & $7(1)$ & $-3(2)$ \\
\hline$C(38)$ & $30(2)$ & $19(2)$ & $21(1)$ & $-3(1)$ & $5(1)$ & $6(1)$ \\
\hline$C(11)$ & $36(2)$ & $42(2)$ & $37(2)$ & $-20(2)$ & $7(2)$ & $4(2)$ \\
\hline$C(42)$ & $46(2)$ & $36(2)$ & $24(2)$ & $3(1)$ & $10(1)$ & $2(2)$ \\
\hline$C(33)$ & $36(2)$ & $46(2)$ & $35(2)$ & $-14(2)$ & $10(2)$ & $-2(2)$ \\
\hline$C(21)$ & $61(3)$ & $40(2)$ & $60(2)$ & $4(2)$ & $33(2)$ & $-14(2)$ \\
\hline$C(22)$ & $24(2)$ & $36(2)$ & $26(2)$ & $4(1)$ & $5(1)$ & 2(1) \\
\hline $\mathrm{C}(40)$ & $37(2)$ & $24(2)$ & $29(2)$ & $-5(1)$ & $-4(1)$ & $5(1)$ \\
\hline
\end{tabular}




\begin{tabular}{lllllll}
$\mathrm{C}(29)$ & $39(2)$ & $30(2)$ & $50(2)$ & $4(2)$ & $14(2)$ & $-4(2)$ \\
$\mathrm{C}(17)$ & $26(2)$ & $17(2)$ & $23(1)$ & $-1(1)$ & $9(1)$ & $2(1)$ \\
$\mathrm{C}(37)$ & $56(2)$ & $26(2)$ & $29(2)$ & $-1(1)$ & $11(2)$ & $7(2)$ \\
$\mathrm{C}(39)$ & $36(2)$ & $24(2)$ & $26(2)$ & $0(1)$ & $4(1)$ & $4(1)$ \\
$\mathrm{C}(30)$ & $39(2)$ & $21(2)$ & $36(2)$ & $0(1)$ & $6(2)$ & $1(1)$ \\
$\mathrm{C}(28)$ & $41(2)$ & $34(2)$ & $60(2)$ & $1(2)$ & $26(2)$ & $3(2)$ \\
$\mathrm{C}(9)$ & $27(2)$ & $35(2)$ & $19(1)$ & $-4(1)$ & $6(1)$ & $4(1)$ \\
$\mathrm{C}(10)$ & $39(2)$ & $54(2)$ & $23(2)$ & $7(2)$ & $7(1)$ & $10(2)$ \\
$\mathrm{C}(41)$ & $47(2)$ & $36(2)$ & $19(2)$ & $0(1)$ & $3(1)$ & $11(2)$ \\
$\mathrm{C}(2)$ & $23(2)$ & $35(2)$ & $24(2)$ & $6(1)$ & $3(1)$ & $-3(1)$ \\
$\mathrm{C}(24)$ & $31(2)$ & $60(3)$ & $25(2)$ & $-1(2)$ & $3(1)$ & $-13(2)$ \\
$\mathrm{C}(25)$ & $32(2)$ & $50(2)$ & $50(2)$ & $21(2)$ & $13(2)$ & $14(2)$ \\
$\mathrm{C}(18)$ & $35(2)$ & $42(2)$ & $34(2)$ & $2(2)$ & $17(2)$ & $-1(2)$ \\
$\mathrm{C}(36)$ & $75(3)$ & $39(2)$ & $29(2)$ & $-4(2)$ & $22(2)$ & $-1(2)$ \\
$\mathrm{C}(34)$ & $34(2)$ & $81(3)$ & $60(3)$ & $-29(2)$ & $16(2)$ & $0(2)$ \\
$\mathrm{C}(20)$ & $35(2)$ & $52(3)$ & $69(3)$ & $-9(2)$ & $26(2)$ & $-1(2)$ \\
$\mathrm{C}(35)$ & $60(3)$ & $69(3)$ & $55(2)$ & $-26(2)$ & $36(2)$ & $-10(2)$ \\
$\mathrm{C}(19)$ & $47(3)$ & $127(5)$ & $42(2)$ & $14(3)$ & $25(2)$ & $-4(3)$ \\
& & & & & & \\
\hline
\end{tabular}


Table S25. Hydrogen coordinates ( $\left.\times 10^{4}\right)$ and isotropic displacement parameters $\left(\AA^{2} \times 10^{3}\right)$ for $\mathbf{4 b}$.

\begin{tabular}{|c|c|c|c|c|}
\hline & $\mathrm{x}$ & $\mathrm{y}$ & $\mathrm{z}$ & $\mathrm{U}(\mathrm{eq})$ \\
\hline $\mathrm{H}(7 \mathrm{~A})$ & 1005 & 5026 & 5176 & 32 \\
\hline $\mathrm{H}(7 \mathrm{~B})$ & 2222 & 4873 & 5471 & 32 \\
\hline $\mathrm{H}(15)$ & 5653 & 6728 & 5708 & 32 \\
\hline $\mathrm{H}(13)$ & 3698 & 6921 & 3882 & 34 \\
\hline $\mathrm{H}(5)$ & 1672 & 3830 & 6187 & 39 \\
\hline $\mathrm{H}(27)$ & 2979 & 7975 & 5579 & 39 \\
\hline $\mathrm{H}(31)$ & 1296 & 9874 & 5886 & 33 \\
\hline $\mathrm{H}(4)$ & 1436 & 3629 & 7222 & 45 \\
\hline $\mathrm{H}(1 \mathrm{~A})$ & 1570 & 6676 & 7590 & 57 \\
\hline $\mathrm{H}(1 \mathrm{~B})$ & 776 & 6166 & 7858 & 57 \\
\hline $\mathrm{H}(1 \mathrm{C})$ & 377 & 6625 & 7166 & 57 \\
\hline $\mathrm{H}(8 \mathrm{~A})$ & 628 & 6059 & 4354 & 32 \\
\hline $\mathrm{H}(8 \mathrm{~B})$ & 886 & 7005 & 4458 & 32 \\
\hline $\mathrm{H}(23 \mathrm{~A})$ & 3243 & 6005 & 6594 & 46 \\
\hline $\mathrm{H}(23 \mathrm{~B})$ & 4124 & 5461 & 7079 & 46 \\
\hline $\mathrm{H}(23 \mathrm{C})$ & 3557 & 5156 & 6346 & 46 \\
\hline $\mathrm{H}(43)$ & 1176 & 9130 & 4535 & 35 \\
\hline $\mathrm{H}(3)$ & 1124 & 4749 & 7804 & 43 \\
\hline $\mathrm{H}(11 \mathrm{~A})$ & 2467 & 5465 & 3608 & 58 \\
\hline $\mathrm{H}(11 \mathrm{~B})$ & 1260 & 5389 & 3535 & 58 \\
\hline $\mathrm{H}(11 \mathrm{C})$ & 2102 & 5135 & 4210 & 58 \\
\hline $\mathrm{H}(42)$ & 257 & 9313 & 3427 & 42 \\
\hline $\mathrm{H}(33)$ & -1183 & 8868 & 5323 & 47 \\
\hline $\mathrm{H}(21 \mathrm{~A})$ & 5684 & 8384 & 4866 & 76 \\
\hline $\mathrm{H}(21 \mathrm{~B})$ & 6803 & 8142 & 4816 & 76 \\
\hline $\mathrm{H}(21 \mathrm{C})$ & 6432 & 7823 & 5419 & 76 \\
\hline $\mathrm{H}(40)$ & -1920 & 7751 & 3618 & 40 \\
\hline $\mathrm{H}(29)$ & 4166 & 10236 & 5729 & 48 \\
\hline $\mathrm{H}(37)$ & 1214 & 8493 & 6969 & 45 \\
\hline H(39) & -1018 & 7567 & 4728 & 36 \\
\hline
\end{tabular}




\begin{tabular}{lrrrr}
$\mathrm{H}(30)$ & 2653 & 10746 & 5892 & 40 \\
$\mathrm{H}(28)$ & 4334 & 8848 & 5586 & 51 \\
$\mathrm{H}(10 \mathrm{~A})$ & 1663 & 7528 & 3679 & 59 \\
$\mathrm{H}(10 B)$ & 900 & 6856 & 3254 & 59 \\
$\mathrm{H}(10 \mathrm{C})$ & 2072 & 6905 & 3237 & 59 \\
$\mathrm{H}(41)$ & -1274 & 8599 & 2960 & 42 \\
$\mathrm{H}(24 \mathrm{~A})$ & 4607 & 7149 & 6857 & 59 \\
$\mathrm{H}(24 \mathrm{~B})$ & 5688 & 6985 & 6720 & 59 \\
$\mathrm{H}(24 \mathrm{C})$ & 5411 & 6518 & 7304 & 59 \\
$\mathrm{H}(25 \mathrm{~A})$ & 5680 & 5142 & 6810 & 66 \\
$\mathrm{H}(25 \mathrm{~B})$ & 6047 & 5668 & 6288 & 66 \\
$\mathrm{H}(25 \mathrm{C})$ & 5156 & 5005 & 6037 & 66 \\
$\mathrm{H}(36)$ & 212 & 8979 & 7610 & 55 \\
$\mathrm{H}(34)$ & -2180 & 9346 & 5968 & 69 \\
$\mathrm{H}(20 \mathrm{~A})$ & 6802 & 6414 & 5164 & 75 \\
$\mathrm{H}(20 B)$ & 7170 & 6726 & 4558 & 75 \\
$\mathrm{H}(20 \mathrm{C})$ & 6289 & 6050 & 4443 & 75 \\
$\mathrm{H}(35)$ & -1496 & 9366 & 7115 & 68 \\
$\mathrm{H}(19 \mathrm{~A})$ & 5192 & 6941 & 3562 & 104 \\
$\mathrm{H}(19 B)$ & 6068 & 7625 & 3723 & 104 \\
$\mathrm{H}(19 \mathrm{C})$ & 4920 & 7841 & 3731 & 104 \\
\hline & & & &
\end{tabular}


Table S26. Crystal data and structure refinement for 4 e.

Identification code

Empirical formula

Formula weight

Temperature

Wavelength

Crystal system

Space group

Unit cell dimensions

Volume

Z

Density (calculated)

Absorption coefficient

$\mathrm{F}(000)$

Crystal size

Theta range for data collection

Index ranges

Reflections collected

Independent reflections

Completeness to theta $=25.000^{\circ}$

Absorption correction

Max. and min. transmission

Refinement method

Data / restraints / parameters

Goodness-of-fit on $\mathrm{F}^{2}$

Final $\mathrm{R}$ indices [I $>2 \operatorname{sigma}(\mathrm{I})]$

$\mathrm{R}$ indices (all data)

Extinction coefficient

Largest diff. peak and hole

SQUEEZE
$\mathrm{MN}-210$

C48 H52 Cl2 N2 O2 P2 Ru

922.82

$100.0 \mathrm{~K}$

$0.71073 \AA$

Monoclinic

P 21/c

$\mathrm{a}=16.3545(6) \AA$

$\alpha=90^{\circ}$.

$\mathrm{b}=14.7944(4) \AA$

$\beta=98.791(2)^{\circ}$.

$\mathrm{c}=19.3941(7) \AA$

$\gamma=90^{\circ}$.

4637.4(3) $\AA^{3}$

4

$1.322 \mathrm{Mg} / \mathrm{m}^{3}$

$0.561 \mathrm{~mm}^{-1}$

1912

$0.32 \times 0.3 \times 0.27 \mathrm{~mm}^{3}$

1.260 to $28.286^{\circ}$.

$-21<=\mathrm{h}<=21,-19<=\mathrm{k}<=12,-25<=\mathrm{l}<=25$

52460

$11467[\mathrm{R}$ (int) $=0.1152]$

$99.9 \%$

Semi-empirical from equivalents

0.2572 and 0.2273

Full-matrix least-squares on $\mathrm{F}^{2}$

$11467 / 0$ / 522

1.017

$\mathrm{R} 1=0.0412, \mathrm{wR} 2=0.1012$

$\mathrm{R} 1=0.0566, \mathrm{wR} 2=0.1101$

$\mathrm{n} / \mathrm{a}$

0.878 and -1.366 e. $\AA^{-3}$

$88 \mathrm{e} / \mathrm{uc} \quad(2$ pentane on $-1 \quad$ site $)$ 
Table S27. Atomic coordinates ( $\left.\times 10^{4}\right)$ and equivalent isotropic displacement parameters $\left(\AA^{2} \times 10^{3}\right)$ for 4e. $U(e q)$ is defined as one third of the trace of the orthogonalized $U^{i j}$ tensor.

\begin{tabular}{|c|c|c|c|c|}
\hline & $\mathrm{x}$ & $\mathrm{y}$ & z & $\mathrm{U}(\mathrm{eq})$ \\
\hline $\mathrm{Ru}(1)$ & 6633(1) & $3728(1)$ & $6591(1)$ & $12(1)$ \\
\hline $\mathrm{Cl}(1)$ & $6149(1)$ & $4033(1)$ & $7666(1)$ & $16(1)$ \\
\hline $\mathrm{Cl}(2)$ & $6659(1)$ & $3542(1)$ & $5364(1)$ & $17(1)$ \\
\hline $\mathrm{P}(1)$ & $7213(1)$ & $5152(1)$ & $6636(1)$ & $13(1)$ \\
\hline $\mathrm{P}(2)$ & 7764(1) & 2902(1) & $6776(1)$ & $14(1)$ \\
\hline $\mathrm{O}(1)$ & $2307(1)$ & $4778(1)$ & $6922(1)$ & $50(1)$ \\
\hline $\mathrm{N}(1)$ & $5995(1)$ & $2447(1)$ & $6570(1)$ & $16(1)$ \\
\hline $\mathrm{O}(2)$ & $2922(2)$ & $3862(1)$ & $7706(1)$ & $43(1)$ \\
\hline $\mathrm{N}(2)$ & $2875(2)$ & $4263(2)$ & $7146(1)$ & $34(1)$ \\
\hline$C(31)$ & $8608(1)$ & 2861(2) & $7536(1)$ & $13(1)$ \\
\hline$C(32)$ & $9367(1)$ & $3005(2)$ & $7278(1)$ & $15(1)$ \\
\hline$C(35)$ & $9362(1)$ & $3123(2)$ & $8684(1)$ & $16(1)$ \\
\hline$C(36)$ & $8630(1)$ & $2836(2)$ & $8270(1)$ & $14(1)$ \\
\hline$C(8)$ & $8045(2)$ & $5323(2)$ & $5477(1)$ & $19(1)$ \\
\hline$C(1)$ & $6444(2)$ & 5992(2) & $6271(1)$ & $17(1)$ \\
\hline$C(38)$ & $9363(1)$ & 2813(2) & $6508(1)$ & $18(1)$ \\
\hline$C(34)$ & $10076(1)$ & $3377(2)$ & $8428(1)$ & $17(1)$ \\
\hline$C(12)$ & $8876(1)$ & $5644(2)$ & $6582(1)$ & $20(1)$ \\
\hline$C(47)$ & $7645(2)$ & $3195(2)$ & $9134(1)$ & $20(1)$ \\
\hline$C(29)$ & $6447(2)$ & $1677(2)$ & $6620(1)$ & $17(1)$ \\
\hline$C(14)$ & 7491(2) & $6579(2)$ & $7616(1)$ & $22(1)$ \\
\hline$C(13)$ & $7562(1)$ & $5662(2)$ & $7494(1)$ & $14(1)$ \\
\hline$C(7)$ & $8109(1)$ & $5401(2)$ & $6202(1)$ & $14(1)$ \\
\hline$C(40)$ & $10021(2)$ & $3324(2)$ & $6180(1)$ & $25(1)$ \\
\hline$C(33)$ & 10081(1) & $3249(2)$ & $7715(1)$ & $18(1)$ \\
\hline$C(46)$ & $8347(1)$ & 1694(2) & $9153(1)$ & $19(1)$ \\
\hline$C(30)$ & $7344(2)$ & $1759(2)$ & $6547(1)$ & $19(1)$ \\
\hline$C(23)$ & $4053(2)$ & $3383(2)$ & $6889(1)$ & 21(1) \\
\hline$C(6)$ & $6590(2)$ & $6683(2)$ & $5814(1)$ & $26(1)$ \\
\hline$C(11)$ & $9552(2)$ & $5811(2)$ & $6243(1)$ & $23(1)$ \\
\hline$C(18)$ & $7970(2)$ & $5119(2)$ & $8024(1)$ & $20(1)$ \\
\hline
\end{tabular}




\begin{tabular}{|c|c|c|c|c|}
\hline$C(22)$ & $3529(2)$ & $4112(2)$ & $6720(1)$ & $24(1)$ \\
\hline$C(24)$ & $4659(1)$ & $3208(2)$ & $6474(1)$ & $18(1)$ \\
\hline $\mathrm{C}(9)$ & $8720(2)$ & $5487(2)$ & $5143(1)$ & $21(1)$ \\
\hline$C(25)$ & $5173(2)$ & $2384(2)$ & $6599(1)$ & 19(1) \\
\hline$C(15)$ & $7822(2)$ & $6949(2)$ & $8258(2)$ & $28(1)$ \\
\hline$C(26)$ & $4802(2)$ & $1567(2)$ & 6724(1) & $23(1)$ \\
\hline$C(48)$ & $7222(1)$ & $2047(2)$ & $8205(1)$ & 19(1) \\
\hline$C(2)$ & $5690(2)$ & $5987(2)$ & $6525(2)$ & $28(1)$ \\
\hline$C(45)$ & 7957(1) & 2462(2) & $8674(1)$ & $16(1)$ \\
\hline$C(19)$ & $4739(2)$ & $3784(2)$ & 5918(1) & $22(1)$ \\
\hline$C(41)$ & $10833(2)$ & $3752(2)$ & 8901(1) & $23(1)$ \\
\hline$C(5)$ & 6001(2) & 7349(2) & $5628(2)$ & $34(1)$ \\
\hline $\mathrm{C}(28)$ & $6098(2)$ & $838(2)$ & $6728(1)$ & $23(1)$ \\
\hline$C(4)$ & $5263(2)$ & $7341(2)$ & $5888(2)$ & $37(1)$ \\
\hline$C(21)$ & $3593(2)$ & $4685(2)$ & $6166(1)$ & $26(1)$ \\
\hline$C(16)$ & $8230(2)$ & 6404(2) & $8780(1)$ & $29(1)$ \\
\hline$C(37)$ & 8491(1) & $3081(2)$ & $6156(1)$ & $17(1)$ \\
\hline$C(20)$ & $4207(2)$ & $4523(2)$ & $5770(1)$ & $28(1)$ \\
\hline$C(39)$ & $9503(2)$ & $1790(2)$ & $6424(1)$ & $25(1)$ \\
\hline$C(27)$ & $5275(2)$ & $781(2)$ & $6793(1)$ & $25(1)$ \\
\hline$C(10)$ & $9479(2)$ & $5731(2)$ & $5529(1)$ & $23(1)$ \\
\hline$C(17)$ & $8306(2)$ & $5491(2)$ & $8661(1)$ & $26(1)$ \\
\hline$C(3)$ & $5109(2)$ & $6662(2)$ & $6342(2)$ & $38(1)$ \\
\hline$C(42)$ & 11282(3) & $4431(3)$ & $8493(2)$ & $69(1)$ \\
\hline$C(44)$ & $10592(2)$ & $4295(3)$ & $9499(2)$ & $55(1)$ \\
\hline$C(43)$ & $11414(2)$ & $3000(2)$ & $9155(3)$ & $99(2)$ \\
\hline
\end{tabular}


Table S28. Bond lengths $[\AA]$ and angles $\left[{ }^{\circ}\right]$ for $\mathbf{4 e}$.

\begin{tabular}{|c|c|}
\hline $\mathrm{Ru}(1)-\mathrm{Cl}(1)$ & $2.3835(6)$ \\
\hline $\mathrm{Ru}(1)-\mathrm{Cl}(2)$ & $2.4016(6)$ \\
\hline $\mathrm{Ru}(1)-\mathrm{P}(1)$ & $2.3072(6)$ \\
\hline $\mathrm{Ru}(1)-\mathrm{P}(2)$ & $2.2007(6)$ \\
\hline $\mathrm{Ru}(1)-\mathrm{N}(1)$ & $2.1608(19)$ \\
\hline $\mathrm{P}(1)-\mathrm{C}(1)$ & $1.832(2)$ \\
\hline $\mathrm{P}(1)-\mathrm{C}(13)$ & $1.837(2)$ \\
\hline $\mathrm{P}(1)-\mathrm{C}(7)$ & $1.836(2)$ \\
\hline $\mathrm{P}(2)-\mathrm{C}(31)$ & $1.860(2)$ \\
\hline $\mathrm{P}(2)-\mathrm{C}(30)$ & $1.853(2)$ \\
\hline $\mathrm{P}(2)-\mathrm{C}(37)$ & $1.834(2)$ \\
\hline $\mathrm{O}(1)-\mathrm{N}(2)$ & $1.229(3)$ \\
\hline $\mathrm{N}(1)-\mathrm{C}(29)$ & $1.353(3)$ \\
\hline $\mathrm{N}(1)-\mathrm{C}(25)$ & $1.357(3)$ \\
\hline $\mathrm{O}(2)-\mathrm{N}(2)$ & $1.230(3)$ \\
\hline $\mathrm{N}(2)-\mathrm{C}(22)$ & $1.465(3)$ \\
\hline $\mathrm{C}(31)-\mathrm{C}(32)$ & $1.424(3)$ \\
\hline$C(31)-C(36)$ & $1.419(3)$ \\
\hline$C(32)-C(38)$ & $1.520(3)$ \\
\hline$C(32)-C(33)$ & $1.382(3)$ \\
\hline $\mathrm{C}(35)-\mathrm{H}(35)$ & 0.9500 \\
\hline$C(35)-C(36)$ & $1.401(3)$ \\
\hline$C(35)-C(34)$ & $1.389(3)$ \\
\hline$C(36)-C(45)$ & $1.547(3)$ \\
\hline $\mathrm{C}(8)-\mathrm{H}(8)$ & 0.9500 \\
\hline $\mathrm{C}(8)-\mathrm{C}(7)$ & $1.397(3)$ \\
\hline $\mathrm{C}(8)-\mathrm{C}(9)$ & $1.385(3)$ \\
\hline $\mathrm{C}(1)-\mathrm{C}(6)$ & $1.397(3)$ \\
\hline $\mathrm{C}(1)-\mathrm{C}(2)$ & $1.397(3)$ \\
\hline $\mathrm{C}(38)-\mathrm{C}(40)$ & $1.531(3)$ \\
\hline $\mathrm{C}(38)-\mathrm{C}(37)$ & $1.537(3)$ \\
\hline$C(38)-C(39)$ & $1.542(3)$ \\
\hline$C(34)-C(33)$ & $1.397(3)$ \\
\hline$C(34)-C(41)$ & $1.528(3)$ \\
\hline
\end{tabular}




\begin{tabular}{|c|c|}
\hline $\mathrm{C}(12)-\mathrm{H}(12)$ & 0.9500 \\
\hline$C(12)-C(7)$ & $1.400(3)$ \\
\hline$C(12)-C(11)$ & $1.392(3)$ \\
\hline $\mathrm{C}(47)-\mathrm{H}(47 \mathrm{~A})$ & 0.9800 \\
\hline C(47)-H(47B) & 0.9800 \\
\hline $\mathrm{C}(47)-\mathrm{H}(47 \mathrm{C})$ & 0.9800 \\
\hline C(47)-C(45) & $1.540(3)$ \\
\hline C(29)-C(30) & $1.500(3)$ \\
\hline C(29)-C(28) & $1.395(3)$ \\
\hline C(14)-H(14) & 0.9500 \\
\hline C(14)-C(13) & $1.384(3)$ \\
\hline C(14)-C(15) & $1.391(3)$ \\
\hline C(13)-C(18) & $1.391(3)$ \\
\hline $\mathrm{C}(40)-\mathrm{H}(40 \mathrm{~A})$ & 0.9800 \\
\hline $\mathrm{C}(40)-\mathrm{H}(40 \mathrm{~B})$ & 0.9800 \\
\hline $\mathrm{C}(40)-\mathrm{H}(40 \mathrm{C})$ & 0.9800 \\
\hline C(33)-H(33) & 0.9500 \\
\hline $\mathrm{C}(46)-\mathrm{H}(46 \mathrm{~A})$ & 0.9800 \\
\hline C(46)-H(46B) & 0.9800 \\
\hline $\mathrm{C}(46)-\mathrm{H}(46 \mathrm{C})$ & 0.9800 \\
\hline C(46)-C(45) & $1.543(3)$ \\
\hline $\mathrm{C}(30)-\mathrm{H}(30 \mathrm{~A})$ & 0.9900 \\
\hline $\mathrm{C}(30)-\mathrm{H}(30 \mathrm{~B})$ & 0.9900 \\
\hline C(23)-H(23) & 0.9500 \\
\hline$C(23)-C(22)$ & $1.385(4)$ \\
\hline C(23)-C(24) & $1.393(3)$ \\
\hline $\mathrm{C}(6)-\mathrm{H}(6)$ & 0.9500 \\
\hline$C(6)-C(5)$ & $1.386(4)$ \\
\hline $\mathrm{C}(11)-\mathrm{H}(11)$ & 0.9500 \\
\hline C(11)-C(10) & $1.376(3)$ \\
\hline C(18)-H(18) & 0.9500 \\
\hline C(18)-C(17) & $1.387(3)$ \\
\hline$C(22)-C(21)$ & $1.387(4)$ \\
\hline$C(24)-C(25)$ & $1.479(3)$ \\
\hline C(24)-C(19) & $1.395(3)$ \\
\hline $\mathrm{C}(9)-\mathrm{H}(9)$ & 0.9500 \\
\hline
\end{tabular}




\begin{tabular}{|c|c|}
\hline C(9)-C(10) & $1.396(3)$ \\
\hline$C(25)-C(26)$ & $1.391(3)$ \\
\hline $\mathrm{C}(15)-\mathrm{H}(15)$ & 0.9500 \\
\hline$C(15)-C(16)$ & $1.384(4)$ \\
\hline C(26)-H(26) & 0.9500 \\
\hline$C(26)-C(27)$ & $1.391(4)$ \\
\hline $\mathrm{C}(48)-\mathrm{H}(48 \mathrm{~A})$ & 0.9800 \\
\hline $\mathrm{C}(48)-\mathrm{H}(48 \mathrm{~B})$ & 0.9800 \\
\hline $\mathrm{C}(48)-\mathrm{H}(48 \mathrm{C})$ & 0.9800 \\
\hline C(48)-C(45) & $1.522(3)$ \\
\hline $\mathrm{C}(2)-\mathrm{H}(2)$ & 0.9500 \\
\hline$C(2)-C(3)$ & $1.387(4)$ \\
\hline C(19)-H(19) & 0.9500 \\
\hline C(19)-C(20) & $1.399(4)$ \\
\hline $\mathrm{C}(41)-\mathrm{C}(42)$ & $1.534(4)$ \\
\hline C(41)-C(44) & $1.511(4)$ \\
\hline C(41)-C(43) & $1.497(4)$ \\
\hline $\mathrm{C}(5)-\mathrm{H}(5)$ & 0.9500 \\
\hline$C(5)-C(4)$ & $1.378(4)$ \\
\hline $\mathrm{C}(28)-\mathrm{H}(28)$ & 0.9500 \\
\hline C(28)-C(27) & $1.374(3)$ \\
\hline $\mathrm{C}(4)-\mathrm{H}(4)$ & 0.9500 \\
\hline$C(4)-C(3)$ & $1.383(4)$ \\
\hline $\mathrm{C}(21)-\mathrm{H}(21)$ & 0.9500 \\
\hline $\mathrm{C}(21)-\mathrm{C}(20)$ & $1.374(4)$ \\
\hline $\mathrm{C}(16)-\mathrm{H}(16)$ & 0.9500 \\
\hline$C(16)-C(17)$ & $1.378(4)$ \\
\hline C(37)-H(37A) & 0.9900 \\
\hline $\mathrm{C}(37)-\mathrm{H}(37 \mathrm{~B})$ & 0.9900 \\
\hline C(20)-H(20) & 0.9500 \\
\hline C(39)-H(39A) & 0.9800 \\
\hline C(39)-H(39B) & 0.9800 \\
\hline C(39)-H(39C) & 0.9800 \\
\hline C(27)-H(27) & 0.9500 \\
\hline $\mathrm{C}(10)-\mathrm{H}(10)$ & 0.9500 \\
\hline $\mathrm{C}(17)-\mathrm{H}(17)$ & 0.9500 \\
\hline
\end{tabular}




\begin{tabular}{|c|c|}
\hline $\mathrm{C}(3)-\mathrm{H}(3)$ & 0.9500 \\
\hline $\mathrm{C}(42)-\mathrm{H}(42 \mathrm{~A})$ & 0.9800 \\
\hline $\mathrm{C}(42)-\mathrm{H}(42 \mathrm{~B})$ & 0.9800 \\
\hline $\mathrm{C}(42)-\mathrm{H}(42 \mathrm{C})$ & 0.9800 \\
\hline $\mathrm{C}(44)-\mathrm{H}(44 \mathrm{~A})$ & 0.9800 \\
\hline $\mathrm{C}(44)-\mathrm{H}(44 \mathrm{~B})$ & 0.9800 \\
\hline $\mathrm{C}(44)-\mathrm{H}(44 \mathrm{C})$ & 0.9800 \\
\hline $\mathrm{C}(43)-\mathrm{H}(43 \mathrm{~A})$ & 0.9800 \\
\hline C(43)-H(43B) & 0.9800 \\
\hline $\mathrm{C}(43)-\mathrm{H}(43 \mathrm{C})$ & 0.9800 \\
\hline $\mathrm{Cl}(1)-\mathrm{Ru}(1)-\mathrm{Cl}(2)$ & $161.22(2)$ \\
\hline $\mathrm{P}(1)-\mathrm{Ru}(1)-\mathrm{Cl}(1)$ & $89.02(2)$ \\
\hline $\mathrm{P}(1)-\mathrm{Ru}(1)-\mathrm{Cl}(2)$ & $94.21(2)$ \\
\hline $\mathrm{P}(2)-\mathrm{Ru}(1)-\mathrm{Cl}(1)$ & $110.22(2)$ \\
\hline $\mathrm{P}(2)-\mathrm{Ru}(1)-\mathrm{Cl}(2)$ & $87.49(2)$ \\
\hline $\mathrm{P}(2)-\mathrm{Ru}(1)-\mathrm{P}(1)$ & $99.81(2)$ \\
\hline $\mathrm{N}(1)-\mathrm{Ru}(1)-\mathrm{Cl}(1)$ & $87.63(5)$ \\
\hline $\mathrm{N}(1)-\mathrm{Ru}(1)-\mathrm{Cl}(2)$ & $87.85(5)$ \\
\hline $\mathrm{N}(1)-\mathrm{Ru}(1)-\mathrm{P}(1)$ & $175.12(5)$ \\
\hline $\mathrm{N}(1)-\mathrm{Ru}(1)-\mathrm{P}(2)$ & $84.69(5)$ \\
\hline $\mathrm{C}(1)-\mathrm{P}(1)-\mathrm{Ru}(1)$ & $110.54(8)$ \\
\hline $\mathrm{C}(1)-\mathrm{P}(1)-\mathrm{C}(13)$ & $99.89(11)$ \\
\hline $\mathrm{C}(1)-\mathrm{P}(1)-\mathrm{C}(7)$ & $103.60(11)$ \\
\hline $\mathrm{C}(13)-\mathrm{P}(1)-\mathrm{Ru}(1)$ & $118.63(7)$ \\
\hline $\mathrm{C}(7)-\mathrm{P}(1)-\mathrm{Ru}(1)$ & $121.18(7)$ \\
\hline C(7)-P(1)-C(13) & $100.05(10)$ \\
\hline $\mathrm{C}(31)-\mathrm{P}(2)-\mathrm{Ru}(1)$ & $130.57(7)$ \\
\hline $\mathrm{C}(30)-\mathrm{P}(2)-\mathrm{Ru}(1)$ & $101.29(8)$ \\
\hline $\mathrm{C}(30)-\mathrm{P}(2)-\mathrm{C}(31)$ & $111.30(10)$ \\
\hline $\mathrm{C}(37)-\mathrm{P}(2)-\mathrm{Ru}(1)$ & $115.20(8)$ \\
\hline $\mathrm{C}(37)-\mathrm{P}(2)-\mathrm{C}(31)$ & $92.75(10)$ \\
\hline $\mathrm{C}(37)-\mathrm{P}(2)-\mathrm{C}(30)$ & $103.23(11)$ \\
\hline $\mathrm{C}(29)-\mathrm{N}(1)-\mathrm{Ru}(1)$ & $118.66(16)$ \\
\hline $\mathrm{C}(29)-\mathrm{N}(1)-\mathrm{C}(25)$ & $118.2(2)$ \\
\hline $\mathrm{C}(25)-\mathrm{N}(1)-\mathrm{Ru}(1)$ & $122.54(16)$ \\
\hline
\end{tabular}




\begin{tabular}{|c|c|}
\hline $\mathrm{O}(1)-\mathrm{N}(2)-\mathrm{O}(2)$ & $123.6(3)$ \\
\hline $\mathrm{O}(1)-\mathrm{N}(2)-\mathrm{C}(22)$ & $118.3(3)$ \\
\hline $\mathrm{O}(2)-\mathrm{N}(2)-\mathrm{C}(22)$ & $118.1(2)$ \\
\hline $\mathrm{C}(32)-\mathrm{C}(31)-\mathrm{P}(2)$ & $107.31(16)$ \\
\hline C(36)-C(31)-P(2) & $134.30(17)$ \\
\hline $\mathrm{C}(36)-\mathrm{C}(31)-\mathrm{C}(32)$ & $117.8(2)$ \\
\hline $\mathrm{C}(31)-\mathrm{C}(32)-\mathrm{C}(38)$ & $116.4(2)$ \\
\hline$C(33)-C(32)-C(31)$ & $121.6(2)$ \\
\hline $\mathrm{C}(33)-\mathrm{C}(32)-\mathrm{C}(38)$ & $121.9(2)$ \\
\hline $\mathrm{C}(36)-\mathrm{C}(35)-\mathrm{H}(35)$ & 117.6 \\
\hline $\mathrm{C}(34)-\mathrm{C}(35)-\mathrm{H}(35)$ & 117.6 \\
\hline $\mathrm{C}(34)-\mathrm{C}(35)-\mathrm{C}(36)$ & $124.7(2)$ \\
\hline$C(31)-C(36)-C(45)$ & $127.3(2)$ \\
\hline$C(35)-C(36)-C(31)$ & $117.1(2)$ \\
\hline$C(35)-C(36)-C(45)$ & $115.5(2)$ \\
\hline $\mathrm{C}(7)-\mathrm{C}(8)-\mathrm{H}(8)$ & 119.6 \\
\hline $\mathrm{C}(9)-\mathrm{C}(8)-\mathrm{H}(8)$ & 119.6 \\
\hline$C(9)-C(8)-C(7)$ & $120.9(2)$ \\
\hline $\mathrm{C}(6)-\mathrm{C}(1)-\mathrm{P}(1)$ & $124.7(2)$ \\
\hline$C(6)-C(1)-C(2)$ & $118.2(2)$ \\
\hline $\mathrm{C}(2)-\mathrm{C}(1)-\mathrm{P}(1)$ & $116.80(18)$ \\
\hline$C(32)-C(38)-C(40)$ & $114.7(2)$ \\
\hline$C(32)-C(38)-C(37)$ & $104.59(18)$ \\
\hline C(32)-C(38)-C(39) & $107.95(19)$ \\
\hline $\mathrm{C}(40)-\mathrm{C}(38)-\mathrm{C}(37)$ & $110.6(2)$ \\
\hline$C(40)-C(38)-C(39)$ & $108.5(2)$ \\
\hline $\mathrm{C}(37)-\mathrm{C}(38)-\mathrm{C}(39)$ & $110.4(2)$ \\
\hline$C(35)-C(34)-C(33)$ & $116.7(2)$ \\
\hline$C(35)-C(34)-C(41)$ & $122.2(2)$ \\
\hline$C(33)-C(34)-C(41)$ & $121.1(2)$ \\
\hline $\mathrm{C}(7)-\mathrm{C}(12)-\mathrm{H}(12)$ & 119.7 \\
\hline $\mathrm{C}(11)-\mathrm{C}(12)-\mathrm{H}(12)$ & 119.7 \\
\hline$C(11)-C(12)-C(7)$ & $120.5(2)$ \\
\hline $\mathrm{H}(47 \mathrm{~A})-\mathrm{C}(47)-\mathrm{H}(47 \mathrm{~B})$ & 109.5 \\
\hline $\mathrm{H}(47 \mathrm{~A})-\mathrm{C}(47)-\mathrm{H}(47 \mathrm{C})$ & 109.5 \\
\hline H(47B)-C(47)-H(47C) & 109.5 \\
\hline
\end{tabular}




\begin{tabular}{|c|c|}
\hline $\mathrm{C}(45)-\mathrm{C}(47)-\mathrm{H}(47 \mathrm{~A})$ & 109.5 \\
\hline $\mathrm{C}(45)-\mathrm{C}(47)-\mathrm{H}(47 \mathrm{~B})$ & 109.5 \\
\hline C(45)-C(47)-H(47C) & 109.5 \\
\hline N(1)-C(29)-C(30) & $117.2(2)$ \\
\hline $\mathrm{N}(1)-\mathrm{C}(29)-\mathrm{C}(28)$ & $121.9(2)$ \\
\hline $\mathrm{C}(28)-\mathrm{C}(29)-\mathrm{C}(30)$ & $120.9(2)$ \\
\hline $\mathrm{C}(13)-\mathrm{C}(14)-\mathrm{H}(14)$ & 119.8 \\
\hline$C(13)-C(14)-C(15)$ & $120.5(2)$ \\
\hline $\mathrm{C}(15)-\mathrm{C}(14)-\mathrm{H}(14)$ & 119.8 \\
\hline $\mathrm{C}(14)-\mathrm{C}(13)-\mathrm{P}(1)$ & $122.31(18)$ \\
\hline $\mathrm{C}(14)-\mathrm{C}(13)-\mathrm{C}(18)$ & $118.9(2)$ \\
\hline $\mathrm{C}(18)-\mathrm{C}(13)-\mathrm{P}(1)$ & $118.61(17)$ \\
\hline $\mathrm{C}(8)-\mathrm{C}(7)-\mathrm{P}(1)$ & $120.15(18)$ \\
\hline $\mathrm{C}(8)-\mathrm{C}(7)-\mathrm{C}(12)$ & $118.4(2)$ \\
\hline $\mathrm{C}(12)-\mathrm{C}(7)-\mathrm{P}(1)$ & $121.46(17)$ \\
\hline $\mathrm{C}(38)-\mathrm{C}(40)-\mathrm{H}(40 \mathrm{~A})$ & 109.5 \\
\hline $\mathrm{C}(38)-\mathrm{C}(40)-\mathrm{H}(40 \mathrm{~B})$ & 109.5 \\
\hline $\mathrm{C}(38)-\mathrm{C}(40)-\mathrm{H}(40 \mathrm{C})$ & 109.5 \\
\hline $\mathrm{H}(40 \mathrm{~A})-\mathrm{C}(40)-\mathrm{H}(40 \mathrm{~B})$ & 109.5 \\
\hline $\mathrm{H}(40 \mathrm{~A})-\mathrm{C}(40)-\mathrm{H}(40 \mathrm{C})$ & 109.5 \\
\hline $\mathrm{H}(40 \mathrm{~B})-\mathrm{C}(40)-\mathrm{H}(40 \mathrm{C})$ & 109.5 \\
\hline C(32)-C(33)-C(34) & $120.7(2)$ \\
\hline $\mathrm{C}(32)-\mathrm{C}(33)-\mathrm{H}(33)$ & 119.7 \\
\hline C(34)-C(33)-H(33) & 119.7 \\
\hline $\mathrm{H}(46 \mathrm{~A})-\mathrm{C}(46)-\mathrm{H}(46 \mathrm{~B})$ & 109.5 \\
\hline $\mathrm{H}(46 \mathrm{~A})-\mathrm{C}(46)-\mathrm{H}(46 \mathrm{C})$ & 109.5 \\
\hline $\mathrm{H}(46 \mathrm{~B})-\mathrm{C}(46)-\mathrm{H}(46 \mathrm{C})$ & 109.5 \\
\hline $\mathrm{C}(45)-\mathrm{C}(46)-\mathrm{H}(46 \mathrm{~A})$ & 109.5 \\
\hline $\mathrm{C}(45)-\mathrm{C}(46)-\mathrm{H}(46 \mathrm{~B})$ & 109.5 \\
\hline $\mathrm{C}(45)-\mathrm{C}(46)-\mathrm{H}(46 \mathrm{C})$ & 109.5 \\
\hline $\mathrm{P}(2)-\mathrm{C}(30)-\mathrm{H}(30 \mathrm{~A})$ & 109.1 \\
\hline $\mathrm{P}(2)-\mathrm{C}(30)-\mathrm{H}(30 \mathrm{~B})$ & 109.1 \\
\hline C(29)-C(30)-P(2) & $112.55(16)$ \\
\hline $\mathrm{C}(29)-\mathrm{C}(30)-\mathrm{H}(30 \mathrm{~A})$ & 109.1 \\
\hline C(29)-C(30)-H(30B) & 109.1 \\
\hline $\mathrm{H}(30 \mathrm{~A})-\mathrm{C}(30)-\mathrm{H}(30 \mathrm{~B})$ & 107.8 \\
\hline
\end{tabular}




\begin{tabular}{|c|c|}
\hline $\mathrm{C}(22)-\mathrm{C}(23)-\mathrm{H}(23)$ & 120.7 \\
\hline $\mathrm{C}(22)-\mathrm{C}(23)-\mathrm{C}(24)$ & $118.5(2)$ \\
\hline $\mathrm{C}(24)-\mathrm{C}(23)-\mathrm{H}(23)$ & 120.7 \\
\hline $\mathrm{C}(1)-\mathrm{C}(6)-\mathrm{H}(6)$ & 119.8 \\
\hline$C(5)-C(6)-C(1)$ & $120.4(3)$ \\
\hline $\mathrm{C}(5)-\mathrm{C}(6)-\mathrm{H}(6)$ & 119.8 \\
\hline $\mathrm{C}(12)-\mathrm{C}(11)-\mathrm{H}(11)$ & 119.8 \\
\hline$C(10)-C(11)-C(12)$ & $120.5(2)$ \\
\hline $\mathrm{C}(10)-\mathrm{C}(11)-\mathrm{H}(11)$ & 119.8 \\
\hline $\mathrm{C}(13)-\mathrm{C}(18)-\mathrm{H}(18)$ & 119.7 \\
\hline$C(17)-C(18)-C(13)$ & $120.6(2)$ \\
\hline $\mathrm{C}(17)-\mathrm{C}(18)-\mathrm{H}(18)$ & 119.7 \\
\hline $\mathrm{C}(23)-\mathrm{C}(22)-\mathrm{N}(2)$ & $117.7(2)$ \\
\hline$C(23)-C(22)-C(21)$ & $122.9(2)$ \\
\hline $\mathrm{C}(21)-\mathrm{C}(22)-\mathrm{N}(2)$ & $119.4(2)$ \\
\hline$C(23)-C(24)-C(25)$ & $119.7(2)$ \\
\hline$C(23)-C(24)-C(19)$ & $119.4(2)$ \\
\hline$C(19)-C(24)-C(25)$ & $120.8(2)$ \\
\hline $\mathrm{C}(8)-\mathrm{C}(9)-\mathrm{H}(9)$ & 120.0 \\
\hline $\mathrm{C}(8)-\mathrm{C}(9)-\mathrm{C}(10)$ & $120.1(2)$ \\
\hline $\mathrm{C}(10)-\mathrm{C}(9)-\mathrm{H}(9)$ & 120.0 \\
\hline $\mathrm{N}(1)-\mathrm{C}(25)-\mathrm{C}(24)$ & $118.5(2)$ \\
\hline $\mathrm{N}(1)-\mathrm{C}(25)-\mathrm{C}(26)$ & $121.9(2)$ \\
\hline$C(26)-C(25)-C(24)$ & $119.5(2)$ \\
\hline $\mathrm{C}(14)-\mathrm{C}(15)-\mathrm{H}(15)$ & 119.9 \\
\hline$C(16)-C(15)-C(14)$ & $120.2(2)$ \\
\hline $\mathrm{C}(16)-\mathrm{C}(15)-\mathrm{H}(15)$ & 119.9 \\
\hline $\mathrm{C}(25)-\mathrm{C}(26)-\mathrm{H}(26)$ & 120.3 \\
\hline$C(25)-C(26)-C(27)$ & $119.5(2)$ \\
\hline $\mathrm{C}(27)-\mathrm{C}(26)-\mathrm{H}(26)$ & 120.3 \\
\hline $\mathrm{H}(48 \mathrm{~A})-\mathrm{C}(48)-\mathrm{H}(48 \mathrm{~B})$ & 109.5 \\
\hline $\mathrm{H}(48 \mathrm{~A})-\mathrm{C}(48)-\mathrm{H}(48 \mathrm{C})$ & 109.5 \\
\hline $\mathrm{H}(48 \mathrm{~B})-\mathrm{C}(48)-\mathrm{H}(48 \mathrm{C})$ & 109.5 \\
\hline $\mathrm{C}(45)-\mathrm{C}(48)-\mathrm{H}(48 \mathrm{~A})$ & 109.5 \\
\hline $\mathrm{C}(45)-\mathrm{C}(48)-\mathrm{H}(48 \mathrm{~B})$ & 109.5 \\
\hline $\mathrm{C}(45)-\mathrm{C}(48)-\mathrm{H}(48 \mathrm{C})$ & 109.5 \\
\hline
\end{tabular}




\begin{tabular}{|c|c|}
\hline $\mathrm{C}(1)-\mathrm{C}(2)-\mathrm{H}(2)$ & 119.5 \\
\hline $\mathrm{C}(3)-\mathrm{C}(2)-\mathrm{C}(1)$ & $120.9(3)$ \\
\hline $\mathrm{C}(3)-\mathrm{C}(2)-\mathrm{H}(2)$ & 119.5 \\
\hline $\mathrm{C}(47)-\mathrm{C}(45)-\mathrm{C}(36)$ & $111.44(19)$ \\
\hline$C(47)-C(45)-C(46)$ & $108.28(19)$ \\
\hline $\mathrm{C}(46)-\mathrm{C}(45)-\mathrm{C}(36)$ & $107.95(18)$ \\
\hline $\mathrm{C}(48)-\mathrm{C}(45)-\mathrm{C}(36)$ & $113.56(19)$ \\
\hline $\mathrm{C}(48)-\mathrm{C}(45)-\mathrm{C}(47)$ & $109.15(19)$ \\
\hline$C(48)-C(45)-C(46)$ & $106.19(19)$ \\
\hline $\mathrm{C}(24)-\mathrm{C}(19)-\mathrm{H}(19)$ & 119.8 \\
\hline$C(24)-C(19)-C(20)$ & $120.5(2)$ \\
\hline $\mathrm{C}(20)-\mathrm{C}(19)-\mathrm{H}(19)$ & 119.8 \\
\hline$C(34)-C(41)-C(42)$ & $109.7(2)$ \\
\hline $\mathrm{C}(44)-\mathrm{C}(41)-\mathrm{C}(34)$ & $111.8(2)$ \\
\hline$C(44)-C(41)-C(42)$ & $104.5(3)$ \\
\hline $\mathrm{C}(43)-\mathrm{C}(41)-\mathrm{C}(34)$ & $110.0(2)$ \\
\hline$C(43)-C(41)-C(42)$ & $109.0(3)$ \\
\hline$C(43)-C(41)-C(44)$ & 111.7(3) \\
\hline $\mathrm{C}(6)-\mathrm{C}(5)-\mathrm{H}(5)$ & 119.6 \\
\hline$C(4)-C(5)-C(6)$ & $120.7(3)$ \\
\hline $\mathrm{C}(4)-\mathrm{C}(5)-\mathrm{H}(5)$ & 119.6 \\
\hline $\mathrm{C}(29)-\mathrm{C}(28)-\mathrm{H}(28)$ & 120.1 \\
\hline $\mathrm{C}(27)-\mathrm{C}(28)-\mathrm{C}(29)$ & $119.8(2)$ \\
\hline $\mathrm{C}(27)-\mathrm{C}(28)-\mathrm{H}(28)$ & 120.1 \\
\hline $\mathrm{C}(5)-\mathrm{C}(4)-\mathrm{H}(4)$ & 120.2 \\
\hline C(5)-C(4)-C(3) & $119.6(3)$ \\
\hline $\mathrm{C}(3)-\mathrm{C}(4)-\mathrm{H}(4)$ & 120.2 \\
\hline $\mathrm{C}(22)-\mathrm{C}(21)-\mathrm{H}(21)$ & 120.9 \\
\hline $\mathrm{C}(20)-\mathrm{C}(21)-\mathrm{C}(22)$ & $118.3(2)$ \\
\hline $\mathrm{C}(20)-\mathrm{C}(21)-\mathrm{H}(21)$ & 120.9 \\
\hline $\mathrm{C}(15)-\mathrm{C}(16)-\mathrm{H}(16)$ & 120.2 \\
\hline$C(17)-C(16)-C(15)$ & $119.7(2)$ \\
\hline $\mathrm{C}(17)-\mathrm{C}(16)-\mathrm{H}(16)$ & 120.2 \\
\hline $\mathrm{P}(2)-\mathrm{C}(37)-\mathrm{H}(37 \mathrm{~A})$ & 109.9 \\
\hline $\mathrm{P}(2)-\mathrm{C}(37)-\mathrm{H}(37 \mathrm{~B})$ & 109.9 \\
\hline $\mathrm{C}(38)-\mathrm{C}(37)-\mathrm{P}(2)$ & $108.83(16)$ \\
\hline
\end{tabular}




\begin{tabular}{|c|c|}
\hline $\mathrm{C}(38)-\mathrm{C}(37)-\mathrm{H}(37 \mathrm{~A})$ & 109.9 \\
\hline $\mathrm{C}(38)-\mathrm{C}(37)-\mathrm{H}(37 \mathrm{~B})$ & 109.9 \\
\hline $\mathrm{H}(37 \mathrm{~A})-\mathrm{C}(37)-\mathrm{H}(37 \mathrm{~B})$ & 108.3 \\
\hline $\mathrm{C}(19)-\mathrm{C}(20)-\mathrm{H}(20)$ & 119.8 \\
\hline$C(21)-C(20)-C(19)$ & $120.4(2)$ \\
\hline $\mathrm{C}(21)-\mathrm{C}(20)-\mathrm{H}(20)$ & 119.8 \\
\hline $\mathrm{C}(38)-\mathrm{C}(39)-\mathrm{H}(39 \mathrm{~A})$ & 109.5 \\
\hline $\mathrm{C}(38)-\mathrm{C}(39)-\mathrm{H}(39 \mathrm{~B})$ & 109.5 \\
\hline C(38)-C(39)-H(39C) & 109.5 \\
\hline $\mathrm{H}(39 \mathrm{~A})-\mathrm{C}(39)-\mathrm{H}(39 \mathrm{~B})$ & 109.5 \\
\hline $\mathrm{H}(39 \mathrm{~A})-\mathrm{C}(39)-\mathrm{H}(39 \mathrm{C})$ & 109.5 \\
\hline $\mathrm{H}(39 \mathrm{~B})-\mathrm{C}(39)-\mathrm{H}(39 \mathrm{C})$ & 109.5 \\
\hline $\mathrm{C}(26)-\mathrm{C}(27)-\mathrm{H}(27)$ & 120.7 \\
\hline $\mathrm{C}(28)-\mathrm{C}(27)-\mathrm{C}(26)$ & $118.5(2)$ \\
\hline $\mathrm{C}(28)-\mathrm{C}(27)-\mathrm{H}(27)$ & 120.7 \\
\hline $\mathrm{C}(11)-\mathrm{C}(10)-\mathrm{C}(9)$ & $119.7(2)$ \\
\hline $\mathrm{C}(11)-\mathrm{C}(10)-\mathrm{H}(10)$ & 120.2 \\
\hline $\mathrm{C}(9)-\mathrm{C}(10)-\mathrm{H}(10)$ & 120.2 \\
\hline $\mathrm{C}(18)-\mathrm{C}(17)-\mathrm{H}(17)$ & 119.9 \\
\hline$C(16)-C(17)-C(18)$ & $120.2(2)$ \\
\hline $\mathrm{C}(16)-\mathrm{C}(17)-\mathrm{H}(17)$ & 119.9 \\
\hline $\mathrm{C}(2)-\mathrm{C}(3)-\mathrm{H}(3)$ & 120.0 \\
\hline $\mathrm{C}(4)-\mathrm{C}(3)-\mathrm{C}(2)$ & $120.1(3)$ \\
\hline $\mathrm{C}(4)-\mathrm{C}(3)-\mathrm{H}(3)$ & 120.0 \\
\hline $\mathrm{C}(41)-\mathrm{C}(42)-\mathrm{H}(42 \mathrm{~A})$ & 109.5 \\
\hline $\mathrm{C}(41)-\mathrm{C}(42)-\mathrm{H}(42 \mathrm{~B})$ & 109.5 \\
\hline $\mathrm{C}(41)-\mathrm{C}(42)-\mathrm{H}(42 \mathrm{C})$ & 109.5 \\
\hline $\mathrm{H}(42 \mathrm{~A})-\mathrm{C}(42)-\mathrm{H}(42 \mathrm{~B})$ & 109.5 \\
\hline $\mathrm{H}(42 \mathrm{~A})-\mathrm{C}(42)-\mathrm{H}(42 \mathrm{C})$ & 109.5 \\
\hline $\mathrm{H}(42 \mathrm{~B})-\mathrm{C}(42)-\mathrm{H}(42 \mathrm{C})$ & 109.5 \\
\hline $\mathrm{C}(41)-\mathrm{C}(44)-\mathrm{H}(44 \mathrm{~A})$ & 109.5 \\
\hline $\mathrm{C}(41)-\mathrm{C}(44)-\mathrm{H}(44 \mathrm{~B})$ & 109.5 \\
\hline $\mathrm{C}(41)-\mathrm{C}(44)-\mathrm{H}(44 \mathrm{C})$ & 109.5 \\
\hline $\mathrm{H}(44 \mathrm{~A})-\mathrm{C}(44)-\mathrm{H}(44 \mathrm{~B})$ & 109.5 \\
\hline $\mathrm{H}(44 \mathrm{~A})-\mathrm{C}(44)-\mathrm{H}(44 \mathrm{C})$ & 109.5 \\
\hline $\mathrm{H}(44 \mathrm{~B})-\mathrm{C}(44)-\mathrm{H}(44 \mathrm{C})$ & 109.5 \\
\hline
\end{tabular}




$\begin{array}{ll}\text { C(41)-C(43)-H(43A) } & 109.5 \\ \text { C(41)-C(43)-H(43B) } & 109.5 \\ \text { C(41)-C(43)-H(43C) } & 109.5 \\ \text { H(43A)-C(43)-H(43B) } & 109.5 \\ \text { H(43A)-C(43)-H(43C) } & 109.5 \\ \text { H(43B)-C(43)-H(43C) } & 109.5\end{array}$

Symmetry transformations used to generate equivalent atoms: 
Table S29. Anisotropic displacement parameters $\left(\AA^{2} \times 10^{3}\right)$ for 4 e. The anisotropic displacement factor exponent takes the form: $-2 \pi^{2}\left[h^{2} a^{* 2} U^{11}+\ldots+2 h k a^{*} b^{*} U^{12}\right]$

\begin{tabular}{|c|c|c|c|c|c|c|}
\hline & $\mathrm{U}^{11}$ & $\mathrm{U}^{22}$ & $\mathrm{U}^{33}$ & $\mathrm{U}^{23}$ & $\mathrm{U}^{13}$ & $\mathrm{U}^{12}$ \\
\hline $\mathrm{Ru}(1)$ & $8(1)$ & $12(1)$ & $17(1)$ & $2(1)$ & $4(1)$ & $0(1)$ \\
\hline $\mathrm{Cl}(1)$ & $12(1)$ & $18(1)$ & 19(1) & $2(1)$ & $6(1)$ & $2(1)$ \\
\hline $\mathrm{Cl}(2)$ & $14(1)$ & $20(1)$ & $17(1)$ & $1(1)$ & $4(1)$ & $0(1)$ \\
\hline $\mathrm{P}(1)$ & $9(1)$ & $14(1)$ & $17(1)$ & $2(1)$ & $3(1)$ & $0(1)$ \\
\hline $\mathrm{P}(2)$ & $11(1)$ & $14(1)$ & $17(1)$ & $2(1)$ & $5(1)$ & $3(1)$ \\
\hline $\mathrm{O}(1)$ & $33(1)$ & $32(1)$ & $90(2)$ & $7(1)$ & $29(1)$ & $12(1)$ \\
\hline $\mathrm{N}(1)$ & 13(1) & $16(1)$ & $18(1)$ & $2(1)$ & $4(1)$ & $-2(1)$ \\
\hline $\mathrm{O}(2)$ & $50(2)$ & $32(1)$ & $55(2)$ & $-2(1)$ & $31(1)$ & $0(1)$ \\
\hline $\mathrm{N}(2)$ & 31(1) & $17(1)$ & $59(2)$ & $-7(1)$ & $18(1)$ & $-4(1)$ \\
\hline $\mathrm{C}(31)$ & $9(1)$ & $13(1)$ & $19(1)$ & $3(1)$ & $6(1)$ & $4(1)$ \\
\hline$C(32)$ & $13(1)$ & $14(1)$ & $20(1)$ & $7(1)$ & $7(1)$ & $4(1)$ \\
\hline$C(35)$ & $14(1)$ & $19(1)$ & $16(1)$ & $3(1)$ & $4(1)$ & $3(1)$ \\
\hline$C(36)$ & $12(1)$ & $12(1)$ & $20(1)$ & $5(1)$ & $6(1)$ & $4(1)$ \\
\hline C(8) & $18(1)$ & $16(1)$ & $24(1)$ & $0(1)$ & $5(1)$ & $-3(1)$ \\
\hline $\mathrm{C}(1)$ & $16(1)$ & $12(1)$ & $22(1)$ & $-1(1)$ & $-1(1)$ & $-1(1)$ \\
\hline $\mathrm{C}(38)$ & $15(1)$ & 21(1) & $21(1)$ & $3(1)$ & $9(1)$ & $5(1)$ \\
\hline$C(34)$ & $12(1)$ & $16(1)$ & $24(1)$ & $3(1)$ & $5(1)$ & $3(1)$ \\
\hline$C(12)$ & $14(1)$ & $22(1)$ & $25(1)$ & $1(1)$ & $7(1)$ & $0(1)$ \\
\hline $\mathrm{C}(47)$ & $16(1)$ & 21(1) & $24(1)$ & $4(1)$ & $9(1)$ & $3(1)$ \\
\hline$C(29)$ & 21(1) & $13(1)$ & $18(1)$ & $0(1)$ & $4(1)$ & $-1(1)$ \\
\hline$C(14)$ & $16(1)$ & $20(1)$ & $28(1)$ & $-3(1)$ & $0(1)$ & $1(1)$ \\
\hline$C(13)$ & $10(1)$ & $15(1)$ & $19(1)$ & $-1(1)$ & $6(1)$ & $-3(1)$ \\
\hline$C(7)$ & $13(1)$ & 11(1) & $21(1)$ & $3(1)$ & $7(1)$ & $-1(1)$ \\
\hline $\mathrm{C}(40)$ & $18(1)$ & $35(2)$ & $25(1)$ & $6(1)$ & $12(1)$ & $4(1)$ \\
\hline$C(33)$ & $10(1)$ & 21(1) & $24(1)$ & $7(1)$ & $9(1)$ & $4(1)$ \\
\hline $\mathrm{C}(46)$ & $14(1)$ & 21(1) & $22(1)$ & $7(1)$ & $7(1)$ & $4(1)$ \\
\hline $\mathrm{C}(30)$ & $16(1)$ & $14(1)$ & $27(1)$ & $0(1)$ & $5(1)$ & $1(1)$ \\
\hline$C(23)$ & $17(1)$ & $16(1)$ & $31(1)$ & $-1(1)$ & $4(1)$ & $-6(1)$ \\
\hline$C(6)$ & $29(2)$ & $18(1)$ & $32(1)$ & $6(1)$ & $7(1)$ & 1(1) \\
\hline $\mathrm{C}(11)$ & $13(1)$ & $24(1)$ & $32(1)$ & $-1(1)$ & $7(1)$ & $-3(1)$ \\
\hline$C(18)$ & $20(1)$ & $19(1)$ & $23(1)$ & $0(1)$ & $5(1)$ & $0(1)$ \\
\hline
\end{tabular}




\begin{tabular}{|c|c|c|c|c|c|c|}
\hline$C(22)$ & $16(1)$ & $20(1)$ & $37(2)$ & $-7(1)$ & $8(1)$ & $-6(1)$ \\
\hline$C(24)$ & $13(1)$ & $16(1)$ & $25(1)$ & $-1(1)$ & $0(1)$ & $-7(1)$ \\
\hline C(9) & $28(1)$ & $16(1)$ & $22(1)$ & $0(1)$ & $11(1)$ & $-4(1)$ \\
\hline$C(25)$ & $15(1)$ & $20(1)$ & $21(1)$ & $1(1)$ & $4(1)$ & $-1(1)$ \\
\hline$C(15)$ & $27(2)$ & $18(1)$ & $40(2)$ & $-9(1)$ & $2(1)$ & $-2(1)$ \\
\hline$C(26)$ & $17(1)$ & $23(1)$ & $31(1)$ & $0(1)$ & $8(1)$ & $-6(1)$ \\
\hline $\mathrm{C}(48)$ & $15(1)$ & $17(1)$ & $26(1)$ & $8(1)$ & $8(1)$ & $0(1)$ \\
\hline$C(2)$ & $15(1)$ & $22(1)$ & $44(2)$ & $9(1)$ & $2(1)$ & $0(1)$ \\
\hline$C(45)$ & 11(1) & $18(1)$ & $19(1)$ & $6(1)$ & $6(1)$ & $3(1)$ \\
\hline$C(19)$ & $13(1)$ & $28(2)$ & $26(1)$ & $5(1)$ & $2(1)$ & $-4(1)$ \\
\hline $\mathrm{C}(41)$ & $15(1)$ & $24(1)$ & $30(1)$ & $0(1)$ & $4(1)$ & $-1(1)$ \\
\hline$C(5)$ & $36(2)$ & $22(2)$ & $43(2)$ & $13(1)$ & $-1(1)$ & $7(1)$ \\
\hline$C(28)$ & $26(1)$ & $14(1)$ & $29(1)$ & $1(1)$ & $6(1)$ & $-2(1)$ \\
\hline$C(4)$ & $26(2)$ & $23(2)$ & $57(2)$ & $6(1)$ & $-8(1)$ & $8(1)$ \\
\hline $\mathrm{C}(21)$ & $16(1)$ & $20(1)$ & $38(2)$ & $-1(1)$ & $-2(1)$ & $2(1)$ \\
\hline$C(16)$ & $31(2)$ & $30(2)$ & $25(1)$ & $-7(1)$ & $1(1)$ & $-9(1)$ \\
\hline$C(37)$ & $14(1)$ & $19(1)$ & $20(1)$ & $3(1)$ & $5(1)$ & $2(1)$ \\
\hline$C(20)$ & $22(1)$ & $27(2)$ & $33(2)$ & $9(1)$ & $-2(1)$ & $-2(1)$ \\
\hline $\mathrm{C}(39)$ & $24(1)$ & $23(2)$ & $32(1)$ & $1(1)$ & $12(1)$ & $9(1)$ \\
\hline$C(27)$ & $30(2)$ & $17(1)$ & $30(1)$ & $2(1)$ & $9(1)$ & $-9(1)$ \\
\hline$C(10)$ & $21(1)$ & $19(1)$ & $34(1)$ & $1(1)$ & $18(1)$ & $-4(1)$ \\
\hline$C(17)$ & $30(2)$ & $26(2)$ & $21(1)$ & $3(1)$ & $-1(1)$ & $-4(1)$ \\
\hline$C(3)$ & $13(1)$ & $33(2)$ & $68(2)$ & $7(2)$ & $4(1)$ & $6(1)$ \\
\hline $\mathrm{C}(42)$ & $87(3)$ & $76(3)$ & $46(2)$ & $-16(2)$ & $16(2)$ & $-66(2)$ \\
\hline$C(44)$ & $26(2)$ & $103(3)$ & $36(2)$ & $-28(2)$ & $4(1)$ & $-13(2)$ \\
\hline $\mathrm{C}(43)$ & $54(3)$ & $30(2)$ & $183(5)$ & $-25(2)$ & $-79(3)$ & $15(2)$ \\
\hline
\end{tabular}


Table S30. Hydrogen coordinates ( $\left.\times 10^{4}\right)$ and isotropic displacement parameters $\left(\AA^{2} \times 10^{3}\right)$ for $4 \mathbf{e}$.

\begin{tabular}{|c|c|c|c|c|}
\hline & $\mathrm{x}$ & $\mathrm{y}$ & z & $\mathrm{U}(\mathrm{eq})$ \\
\hline $\mathrm{H}(35)$ & 9370 & 3145 & 9174 & 19 \\
\hline $\mathrm{H}(8)$ & 7531 & 5154 & 5211 & 23 \\
\hline $\mathrm{H}(12)$ & 8934 & 5695 & 7075 & 24 \\
\hline $\mathrm{H}(47 \mathrm{~A})$ & 8115 & 3456 & 9444 & 29 \\
\hline $\mathrm{H}(47 \mathrm{~B})$ & 7257 & 2925 & 9413 & 29 \\
\hline $\mathrm{H}(47 \mathrm{C})$ & 7363 & 3671 & 8836 & 29 \\
\hline $\mathrm{H}(14)$ & 7214 & 6958 & 7259 & 26 \\
\hline $\mathrm{H}(40 \mathrm{~A})$ & 9958 & 3975 & 6249 & 38 \\
\hline $\mathrm{H}(40 \mathrm{~B})$ & 9953 & 3193 & 5679 & 38 \\
\hline $\mathrm{H}(40 \mathrm{C})$ & 10573 & 3133 & 6401 & 38 \\
\hline $\mathrm{H}(33)$ & 10581 & 3331 & 7529 & 21 \\
\hline $\mathrm{H}(46 \mathrm{~A})$ & 8539 & 1214 & 8868 & 28 \\
\hline $\mathrm{H}(46 \mathrm{~B})$ & 7933 & 1448 & 9417 & 28 \\
\hline $\mathrm{H}(46 \mathrm{C})$ & 8816 & 1931 & 9477 & 28 \\
\hline $\mathrm{H}(30 \mathrm{~A})$ & 7412 & 1620 & 6060 & 22 \\
\hline $\mathrm{H}(30 \mathrm{~B})$ & 7666 & 1308 & 6854 & 22 \\
\hline $\mathrm{H}(23)$ & 4000 & 3011 & 7279 & 26 \\
\hline $\mathrm{H}(6)$ & 7096 & 6697 & 5629 & 31 \\
\hline $\mathrm{H}(11)$ & 10067 & 5982 & 6506 & 27 \\
\hline $\mathrm{H}(18)$ & 8018 & 4488 & 7948 & 24 \\
\hline $\mathrm{H}(9)$ & 8666 & 5435 & 4650 & 26 \\
\hline $\mathrm{H}(15)$ & 7769 & 7579 & 8337 & 34 \\
\hline $\mathrm{H}(26)$ & 4230 & 1545 & 6761 & 28 \\
\hline $\mathrm{H}(48 \mathrm{~A})$ & 6951 & 2510 & 7887 & 28 \\
\hline $\mathrm{H}(48 \mathrm{~B})$ & 6827 & 1811 & 8492 & 28 \\
\hline $\mathrm{H}(48 \mathrm{C})$ & 7414 & 1554 & 7933 & 28 \\
\hline $\mathrm{H}(2)$ & 5573 & 5515 & 6827 & 33 \\
\hline H(19) & 5157 & 3673 & 5638 & 27 \\
\hline $\mathrm{H}(5)$ & 6108 & 7817 & 5318 & 41 \\
\hline $\mathrm{H}(28)$ & 6429 & 308 & 6755 & 28 \\
\hline
\end{tabular}




\begin{tabular}{|c|c|c|c|c|}
\hline $\mathrm{H}(4)$ & 4862 & 7799 & 5757 & 44 \\
\hline $\mathrm{H}(21)$ & 3222 & 5178 & 6062 & 31 \\
\hline $\mathrm{H}(16)$ & 8455 & 6656 & 9218 & 35 \\
\hline $\mathrm{H}(37 \mathrm{~A})$ & 8326 & 2709 & 5733 & 20 \\
\hline $\mathrm{H}(37 \mathrm{~B})$ & 8485 & 3724 & 6014 & 20 \\
\hline $\mathrm{H}(20)$ & 4271 & 4915 & 5394 & 33 \\
\hline $\mathrm{H}(39 \mathrm{~A})$ & 10050 & 1624 & 6672 & 38 \\
\hline $\mathrm{H}(39 \mathrm{~B})$ & 9472 & 1644 & 5928 & 38 \\
\hline $\mathrm{H}(39 \mathrm{C})$ & 9076 & 1453 & 6619 & 38 \\
\hline $\mathrm{H}(27)$ & 5034 & 217 & 6884 & 30 \\
\hline $\mathrm{H}(10)$ & 9943 & 5841 & 5301 & 28 \\
\hline $\mathrm{H}(17)$ & 8590 & 5116 & 9017 & 32 \\
\hline $\mathrm{H}(3)$ & 4604 & 6658 & 6528 & 46 \\
\hline $\mathrm{H}(42 \mathrm{~A})$ & 11551 & 4108 & 8148 & 104 \\
\hline $\mathrm{H}(42 \mathrm{~B})$ & 11701 & 4755 & 8817 & 104 \\
\hline $\mathrm{H}(42 \mathrm{C})$ & 10882 & 4864 & 8255 & 104 \\
\hline $\mathrm{H}(44 \mathrm{~A})$ & 10166 & 4737 & 9318 & 83 \\
\hline $\mathrm{H}(44 \mathrm{~B})$ & 11079 & 4611 & 9741 & 83 \\
\hline $\mathrm{H}(44 \mathrm{C})$ & 10374 & 3888 & 9826 & 83 \\
\hline $\mathrm{H}(43 \mathrm{~A})$ & 11129 & 2558 & 9409 & 149 \\
\hline $\mathrm{H}(43 \mathrm{~B})$ & 11891 & 3245 & 9466 & 149 \\
\hline $\mathrm{H}(43 \mathrm{C})$ & 11604 & 2705 & 8755 & 149 \\
\hline
\end{tabular}


Table S31. Crystal data and structure refinement for $\mathbf{5 a}$.

Identification code

Empirical formula

Formula weight

Temperature

Wavelength

Crystal system

Space group

Unit cell dimensions

Volume

Z, Z'

Density (calculated)

Absorption coefficient

$\mathrm{F}(000)$

Crystal size

Theta range for data collection

Index ranges

Reflections collected

Independent reflections

Completeness to theta $=25.242^{\circ}$

Absorption correction

Max. and min. transmission

Refinement method

Data / restraints / parameters

Goodness-of-fit on $\mathrm{F}^{2}$

Final $\mathrm{R}$ indices [I $>2 \operatorname{sigma}(\mathrm{I})]$

$\mathrm{R}$ indices (all data)

Largest diff. peak and hole

Twinning
MN-292

C43 H49 Cl5 D N P2 Ru (with $\mathrm{CDCl}_{3}$ )

922.10

$100.0 \mathrm{~K}$

$0.71073 \AA$

Triclinic

P-1

$\mathrm{a}=13.8456(15) \AA$

$\alpha=100.556(3)^{\circ}$.

$\mathrm{b}=17.1964(19) \AA$

$\beta=91.015(3)^{\circ}$.

$\mathrm{c}=18.546(2) \AA$

$4338.0(8) \AA^{3}$

4, 2

$1.412 \mathrm{Mg} / \mathrm{m}^{3}$

$0.774 \mathrm{~mm}^{-1}$

1896

$0.29 \times 0.22 \times 0.18 \mathrm{~mm}^{3}$

1.205 to $25.386^{\circ}$.

$-15<=\mathrm{h}<=16,-20<=\mathrm{k}<=20,-22<=1<=22$

38139

$14139[\mathrm{R}(\mathrm{int})=0.046]$

$99.9 \%$

Semi-empirical from equivalents

0.2590 and 0.1721

Full-matrix least-squares on $\mathrm{F}^{2}$

14139 / 0 / 954

1.035

$\mathrm{R} 1=0.0578, \mathrm{wR} 2=0.1147$

$\mathrm{R} 1=0.1049, \mathrm{wR} 2=0.1349$

0.895 and -1.206 e. $\AA^{-}$

rotational

twin

ca.

$50 / 50$ 
Table S32. Atomic coordinates ( x 10 $0^{4}$ ) and equivalent isotropic displacement parameters $\left(\AA^{2} \mathrm{x} 10^{3}\right)$ for 5a. $U(e q)$ is defined as one third of the trace of the orthogonalized $U^{i j}$ tensor.

\begin{tabular}{|c|c|c|c|c|}
\hline & $\mathrm{x}$ & $\mathrm{y}$ & $\mathrm{z}$ & $\mathrm{U}(\mathrm{eq})$ \\
\hline $\mathrm{Ru}(1)$ & 7333(1) & $4779(1)$ & $6589(1)$ & $12(1)$ \\
\hline $\mathrm{Ru}(2)$ & $7328(1)$ & $-203(1)$ & $6688(1)$ & $14(1)$ \\
\hline $\mathrm{Cl}(1)$ & $7443(1)$ & $3823(1)$ & $5426(1)$ & $17(1)$ \\
\hline $\mathrm{Cl}(2)$ & $6857(1)$ & $3702(1)$ & $7205(1)$ & $17(1)$ \\
\hline $\mathrm{Cl}(3)$ & $7829(1)$ & $-1286(1)$ & $7275(1)$ & $18(1)$ \\
\hline $\mathrm{P}(1)$ & $8798(1)$ & $4890(1)$ & $7141(1)$ & $14(1)$ \\
\hline $\mathrm{P}(4)$ & $8088(1)$ & $802(1)$ & $7447(1)$ & $14(1)$ \\
\hline $\mathrm{P}(3)$ & $5915(1)$ & $-145(1)$ & $7268(1)$ & $15(1)$ \\
\hline $\mathrm{Cl}(4)$ & 7083(1) & $-1152(1)$ & $5529(1)$ & $22(1)$ \\
\hline $\mathrm{Cl}(9)$ & $9167(2)$ & $7817(1)$ & $8811(1)$ & $36(1)$ \\
\hline $\mathrm{Cl}(10)$ & $10877(2)$ & $8569(1)$ & $8366(1)$ & $51(1)$ \\
\hline $\mathrm{Cl}(5)$ & $5665(2)$ & $2789(1)$ & $8833(1)$ & $36(1)$ \\
\hline $\mathrm{P}(2)$ & $6663(1)$ & $5761(1)$ & $7362(1)$ & $13(1)$ \\
\hline $\mathrm{Cl}(6)$ & $4071(2)$ & $3708(1)$ & $8493(1)$ & $53(1)$ \\
\hline $\mathrm{Cl}(8)$ & $9469(2)$ & $9497(1)$ & $9224(1)$ & $61(1)$ \\
\hline $\mathrm{Cl}(7)$ & $5711(2)$ & $4467(1)$ & $9321(1)$ & $63(1)$ \\
\hline$C(57)$ & $10476(5)$ & $1382(4)$ & $8694(4)$ & $17(2)$ \\
\hline $\mathrm{N}(2)$ & $6970(4)$ & $673(3)$ & $6082(3)$ & $14(1)$ \\
\hline$C(20)$ & 2101(5) & $5280(4)$ & $8678(4)$ & $26(2)$ \\
\hline$C(13)$ & $3570(5)$ & $5963(4)$ & $8285(4)$ & $15(2)$ \\
\hline$C(56)$ & $11266(5)$ & $1046(4)$ & $8319(4)$ & $15(2)$ \\
\hline$C(55)$ & $11086(5)$ & $603(4)$ & $7621(4)$ & $18(2)$ \\
\hline$C(23)$ & $4095(5)$ & $4027(3)$ & $6501(4)$ & $24(2)$ \\
\hline$C(66)$ & $12742(5)$ & $359(4)$ & $8626(4)$ & $27(2)$ \\
\hline$C(79)$ & $5935(5)$ & $124(4)$ & $8273(4)$ & $16(2)$ \\
\hline$C(63)$ & $12295(5)$ & $1173(4)$ & $8630(4)$ & $21(2)$ \\
\hline$C(18)$ & $2600(5)$ & $6612(4)$ & $9368(4)$ & $26(2)$ \\
\hline$C(78)$ & $4470(5)$ & $-1149(4)$ & $7667(4)$ & $24(2)$ \\
\hline$C(19)$ & $1948(5)$ & $6483(4)$ & $8097(4)$ & $29(2)$ \\
\hline$C(28)$ & $10638(5)$ & $6800(4)$ & $6289(4)$ & $27(2)$ \\
\hline$C(21)$ & $4478(5)$ & $4868(4)$ & $6452(3)$ & $15(2)$ \\
\hline
\end{tabular}




\begin{tabular}{|c|c|c|c|c|}
\hline $\mathrm{C}(71)$ & $3740(5)$ & $842(4)$ & $6226(4)$ & $23(2)$ \\
\hline$C(52)$ & $9159(5)$ & $-168(4)$ & $6117(3)$ & $23(2)$ \\
\hline$C(82)$ & $6037(5)$ & 494(4) & $9805(4)$ & $23(2)$ \\
\hline$C(64)$ & $12342(5)$ & 1632(4) & $9422(4)$ & $28(2)$ \\
\hline$C(73)$ & $5154(5)$ & $-1063(4)$ & $7152(4)$ & $18(2)$ \\
\hline C(69) & 4852(5) & 1888(4) & 6767(4) & $22(2)$ \\
\hline$C(8)$ & $6196(5)$ & 6631(3) & $8693(3)$ & $14(2)$ \\
\hline$C(62)$ & $8675(5)$ & 1608(4) & 9597(3) & $19(2)$ \\
\hline$C(72)$ & $4264(5)$ & $313(4)$ & $6548(4)$ & $19(2)$ \\
\hline $\mathrm{C}(9)$ & $6174(5)$ & $7508(3)$ & $8613(4)$ & $21(2)$ \\
\hline$C(70)$ & $4046(5)$ & $1628(4)$ & $6328(4)$ & $23(2)$ \\
\hline$C(14)$ & $3675(5)$ & $5505(4)$ & $7590(4)$ & $17(2)$ \\
\hline$C(59)$ & $8697(4)$ & 1674(3) & $8777(3)$ & $12(2)$ \\
\hline$C(17)$ & $2566(5)$ & 6082(4) & $8605(4)$ & $17(2)$ \\
\hline$C(50)$ & $10180(5)$ & $460(3)$ & $7279(3)$ & $14(2)$ \\
\hline C(24) & $3780(5)$ & $5255(4)$ & $5983(3)$ & $22(2)$ \\
\hline$C(5)$ & $7273(5)$ & 6395(4) & $6156(3)$ & $15(2)$ \\
\hline$C(49)$ & 9381(5) & $798(4)$ & $7681(3)$ & $16(2)$ \\
\hline$C(41)$ & $10063(5)$ & 2812(4) & $6212(4)$ & $26(2)$ \\
\hline$C(37)$ & $9542(5)$ & 4002(4) & 6991(4) & $17(2)$ \\
\hline$C(10)$ & $6294(5)$ & $6588(4)$ & $9508(3)$ & $20(2)$ \\
\hline$C(58)$ & 9554(5) & 1261(4) & $8390(3)$ & $14(2)$ \\
\hline$C(76)$ & $3909(6)$ & $-2392(4)$ & $6952(4)$ & $32(2)$ \\
\hline$C(65)$ & $12899(5)$ & 1632(4) & $8151(4)$ & $30(2)$ \\
\hline$C(12)$ & $4402(5)$ & 6308(3) & $8640(4)$ & $16(2)$ \\
\hline$C(86)$ & $5325(5)$ & $3709(4)$ & $8624(4)$ & $28(2)$ \\
\hline $\mathrm{D}(86)$ & 5640 & 3785 & 8160 & 34 \\
\hline$C(74)$ & $5225(5)$ & $-1651(4)$ & $6536(4)$ & $22(2)$ \\
\hline$C(84)$ & $6454(5)$ & $-339(4)$ & $8678(4)$ & $20(2)$ \\
\hline$C(80)$ & $5456(5)$ & $764(4)$ & $8652(4)$ & $17(2)$ \\
\hline$C(53)$ & $10586(6)$ & $-841(4)$ & $6503(4)$ & $31(2)$ \\
\hline$C(35)$ & $9454(5)$ & $5958(4)$ & $9286(4)$ & $21(2)$ \\
\hline $\mathrm{N}(1)$ & 7648(4) & $5665(3)$ & $5984(3)$ & $16(1)$ \\
\hline$C(42)$ & $9463(5)$ & $3450(4)$ & $6341(4)$ & $21(2)$ \\
\hline$C(32)$ & $8427(5)$ & $4640(4)$ & $8559(4)$ & $20(2)$ \\
\hline $\mathrm{C}(81)$ & $5506(5)$ & $955(4)$ & $9416(4)$ & $21(2)$ \\
\hline
\end{tabular}




\begin{tabular}{|c|c|c|c|c|}
\hline$C(77)$ & $3848(6)$ & $-1814(4)$ & $7571(5)$ & $35(2)$ \\
\hline$C(30)$ & $9311(5)$ & 6431(4) & $6973(3)$ & $17(2)$ \\
\hline $\mathrm{C}(31)$ & $8883(5)$ & $5136(4)$ & $8148(4)$ & $15(2)$ \\
\hline$C(83)$ & $6501(5)$ & $-151(4)$ & $9439(4)$ & $22(2)$ \\
\hline$C(27)$ & $10943(5)$ & 6023(4) & $6171(4)$ & $25(2)$ \\
\hline$C(26)$ & $10413(5)$ & 5451(4) & $6446(4)$ & $20(2)$ \\
\hline$C(43)$ & $6309(5)$ & $533(4)$ & $5525(3)$ & $18(2)$ \\
\hline $\mathrm{C}(2)$ & $8475(5)$ & 6106(4) & $5005(4)$ & $23(2)$ \\
\hline$C(15)$ & $4555(5)$ & 5374(3) & $7244(3)$ & $13(2)$ \\
\hline$C(16)$ & 5394(4) & $5721(3)$ & $7624(3)$ & $11(2)$ \\
\hline $\mathrm{C}(38)$ & $10238(6)$ & $3898(4)$ & $7504(5)$ & $35(2)$ \\
\hline$C(25)$ & $9589(5)$ & $5643(4)$ & $6848(3)$ & $14(2)$ \\
\hline$C(68)$ & $5384(5)$ & $1355(4)$ & $7074(4)$ & $19(2)$ \\
\hline$C(75)$ & 4596(5) & $-2316(4)$ & $6445(4)$ & $25(2)$ \\
\hline $\mathrm{C}(11)$ & $5303(5)$ & 6195(3) & $8326(3)$ & $12(2)$ \\
\hline $\mathrm{C}(29)$ & $9830(5)$ & 7000(4) & $6696(4)$ & $24(2)$ \\
\hline$C(1)$ & $8253(5)$ & $5542(4)$ & $5410(4)$ & $19(2)$ \\
\hline$C(85)$ & $9635(5)$ & $8672(4)$ & $8544(4)$ & $29(2)$ \\
\hline $\mathrm{D}(85)$ & 9289 & 8747 & 8085 & 34 \\
\hline$C(7)$ & 7049(4) & 6210(3) & $8294(3)$ & $14(2)$ \\
\hline $\mathrm{C}(4)$ & $7457(5)$ & 6979(4) & $5751(4)$ & $19(2)$ \\
\hline$C(51)$ & $10167(5)$ & $-28(4)$ & $6492(4)$ & $17(2)$ \\
\hline$C(34)$ & $8992(5)$ & $5465(4)$ & $9680(4)$ & $24(2)$ \\
\hline$C(54)$ & 10782(6) & $411(4)$ & $5999(4)$ & $28(2)$ \\
\hline C(39) & $10840(6)$ & $3250(5)$ & $7383(5)$ & $47(3)$ \\
\hline $\mathrm{C}(40)$ & $10750(6)$ & 2712(4) & $6738(5)$ & $35(2)$ \\
\hline$C(47)$ & $7384(5)$ & 1413(4) & $6251(4)$ & $15(2)$ \\
\hline$C(6)$ & $6682(5)$ & 6572(3) & $6829(4)$ & $18(2)$ \\
\hline$C(45)$ & $6517(6)$ & $1835(4)$ & $5272(4)$ & $26(2)$ \\
\hline$C(36)$ & $9413(5)$ & $5788(4)$ & $8523(4)$ & $17(2)$ \\
\hline $\mathrm{C}(60)$ & 7801(5) & $1247(4)$ & $8383(3)$ & $16(2)$ \\
\hline$C(3)$ & $8049(6)$ & $6838(4)$ & $5168(4)$ & $27(2)$ \\
\hline$C(67)$ & $5102(5)$ & $553(4)$ & $6963(4)$ & $18(2)$ \\
\hline$C(61)$ & $8749(5)$ & $2558(3)$ & $8718(4)$ & $22(2)$ \\
\hline$C(22)$ & $5437(5)$ & 4784(4) & $6057(3)$ & $23(2)$ \\
\hline$C(48)$ & $8044(5)$ & 1611(4) & 6916(4) & $21(2)$ \\
\hline
\end{tabular}


C(46)

C(33)

C(44)
7182(5)

8477(5)

6061(5)
1985(4)

4797(4)

1088(4)
5846(4)

9318(4)

5122(4)
22(2)

28(2)

22(2) 
Table S33. Bond lengths $[\AA]$ and angles $\left[^{\circ}\right]$ for $\mathbf{5 a}$.

\begin{tabular}{|c|c|}
\hline $\mathrm{Ru}(1)-\mathrm{Cl}(1)$ & $2.4701(15)$ \\
\hline $\mathrm{Ru}(1)-\mathrm{Cl}(2)$ & $2.4293(17)$ \\
\hline $\mathrm{Ru}(1)-\mathrm{P}(1)$ & $2.2439(19)$ \\
\hline $\mathrm{Ru}(1)-\mathrm{P}(2)$ & $2.2397(17)$ \\
\hline $\mathrm{Ru}(1)-\mathrm{N}(1)$ & $2.093(5)$ \\
\hline $\mathrm{Ru}(2)-\mathrm{Cl}(3)$ & $2.4334(17)$ \\
\hline $\mathrm{Ru}(2)-\mathrm{P}(4)$ & $2.2436(17)$ \\
\hline $\mathrm{Ru}(2)-\mathrm{P}(3)$ & $2.2447(19)$ \\
\hline $\mathrm{Ru}(2)-\mathrm{Cl}(4)$ & $2.4612(16)$ \\
\hline $\mathrm{Ru}(2)-\mathrm{N}(2)$ & $2.103(5)$ \\
\hline $\mathrm{P}(1)-\mathrm{C}(37)$ & $1.845(6)$ \\
\hline $\mathrm{P}(1)-\mathrm{C}(31)$ & $1.837(7)$ \\
\hline$P(1)-C(25)$ & $1.837(7)$ \\
\hline $\mathrm{P}(4)-\mathrm{C}(49)$ & $1.835(7)$ \\
\hline$P(4)-C(60)$ & $1.820(6)$ \\
\hline $\mathrm{P}(4)-\mathrm{C}(48)$ & $1.847(7)$ \\
\hline $\mathrm{P}(3)-\mathrm{C}(79)$ & $1.836(7)$ \\
\hline $\mathrm{P}(3)-\mathrm{C}(73)$ & $1.850(7)$ \\
\hline$P(3)-C(67)$ & $1.824(7)$ \\
\hline $\mathrm{Cl}(9)-\mathrm{C}(85)$ & $1.747(7)$ \\
\hline $\mathrm{Cl}(10)-\mathrm{C}(85)$ & $1.765(8)$ \\
\hline $\mathrm{Cl}(5)-\mathrm{C}(86)$ & $1.772(7)$ \\
\hline $\mathrm{P}(2)-\mathrm{C}(16)$ & $1.834(6)$ \\
\hline$P(2)-C(7)$ & $1.824(6)$ \\
\hline$P(2)-C(6)$ & $1.852(7)$ \\
\hline $\mathrm{Cl}(6)-\mathrm{C}(86)$ & $1.748(8)$ \\
\hline $\mathrm{Cl}(8)-\mathrm{C}(85)$ & $1.741(7)$ \\
\hline $\mathrm{Cl}(7)-\mathrm{C}(86)$ & $1.724(7)$ \\
\hline $\mathrm{C}(57)-\mathrm{H}(57)$ & 0.9500 \\
\hline$C(57)-C(56)$ & $1.389(9)$ \\
\hline$C(57)-C(58)$ & $1.381(9)$ \\
\hline $\mathrm{N}(2)-\mathrm{C}(43)$ & $1.352(8)$ \\
\hline $\mathrm{N}(2)-\mathrm{C}(47)$ & $1.361(8)$ \\
\hline $\mathrm{C}(20)-\mathrm{H}(20 \mathrm{~A})$ & 0.9800 \\
\hline
\end{tabular}




\begin{tabular}{|c|c|}
\hline $\mathrm{C}(20)-\mathrm{H}(20 \mathrm{~B})$ & 0.9800 \\
\hline $\mathrm{C}(20)-\mathrm{H}(20 \mathrm{C})$ & 0.9800 \\
\hline C(20)-C(17) & $1.535(9)$ \\
\hline$C(13)-C(14)$ & $1.396(8)$ \\
\hline$C(13)-C(17)$ & $1.525(9)$ \\
\hline$C(13)-C(12)$ & $1.380(9)$ \\
\hline$C(56)-C(55)$ & $1.391(8)$ \\
\hline$C(56)-C(63)$ & $1.522(9)$ \\
\hline $\mathrm{C}(55)-\mathrm{H}(55)$ & 0.9500 \\
\hline$C(55)-C(50)$ & $1.392(9)$ \\
\hline $\mathrm{C}(23)-\mathrm{H}(23 \mathrm{~A})$ & 0.9800 \\
\hline $\mathrm{C}(23)-\mathrm{H}(23 \mathrm{~B})$ & 0.9800 \\
\hline $\mathrm{C}(23)-\mathrm{H}(23 \mathrm{C})$ & 0.9800 \\
\hline$C(23)-C(21)$ & $1.544(9)$ \\
\hline $\mathrm{C}(66)-\mathrm{H}(66 \mathrm{~A})$ & 0.9800 \\
\hline $\mathrm{C}(66)-\mathrm{H}(66 \mathrm{~B})$ & 0.9800 \\
\hline $\mathrm{C}(66)-\mathrm{H}(66 \mathrm{C})$ & 0.9800 \\
\hline$C(66)-C(63)$ & $1.544(9)$ \\
\hline C(79)-C(84) & $1.396(9)$ \\
\hline $\mathrm{C}(79)-\mathrm{C}(80)$ & $1.385(8)$ \\
\hline$C(63)-C(64)$ & $1.534(9)$ \\
\hline$C(63)-C(65)$ & $1.537(10)$ \\
\hline $\mathrm{C}(18)-\mathrm{H}(18 \mathrm{~A})$ & 0.9800 \\
\hline $\mathrm{C}(18)-\mathrm{H}(18 \mathrm{~B})$ & 0.9800 \\
\hline $\mathrm{C}(18)-\mathrm{H}(18 \mathrm{C})$ & 0.9800 \\
\hline C(18)-C(17) & $1.535(8)$ \\
\hline $\mathrm{C}(78)-\mathrm{H}(78)$ & 0.9500 \\
\hline$C(78)-C(73)$ & $1.380(10)$ \\
\hline$C(78)-C(77)$ & $1.395(9)$ \\
\hline $\mathrm{C}(19)-\mathrm{H}(19 \mathrm{~A})$ & 0.9800 \\
\hline C(19)-H(19B) & 0.9800 \\
\hline C(19)-H(19C) & 0.9800 \\
\hline C(19)-C(17) & $1.528(9)$ \\
\hline $\mathrm{C}(28)-\mathrm{H}(28)$ & 0.9500 \\
\hline$C(28)-C(27)$ & $1.392(9)$ \\
\hline $\mathrm{C}(28)-\mathrm{C}(29)$ & $1.377(10)$ \\
\hline
\end{tabular}




\begin{tabular}{|c|c|}
\hline C(21)-C(24) & $1.533(9)$ \\
\hline$C(21)-C(15)$ & $1.565(8)$ \\
\hline$C(21)-C(22)$ & $1.525(9)$ \\
\hline $\mathrm{C}(71)-\mathrm{H}(71)$ & 0.9500 \\
\hline$C(71)-C(72)$ & $1.389(9)$ \\
\hline$C(71)-C(70)$ & $1.383(9)$ \\
\hline $\mathrm{C}(52)-\mathrm{H}(52 \mathrm{~A})$ & 0.9800 \\
\hline $\mathrm{C}(52)-\mathrm{H}(52 \mathrm{~B})$ & 0.9800 \\
\hline $\mathrm{C}(52)-\mathrm{H}(52 \mathrm{C})$ & 0.9800 \\
\hline $\mathrm{C}(52)-\mathrm{C}(51)$ & $1.541(9)$ \\
\hline C(82)-H(82) & 0.9500 \\
\hline $\mathrm{C}(82)-\mathrm{C}(81)$ & $1.383(9)$ \\
\hline C(82)-C(83) & $1.373(9)$ \\
\hline $\mathrm{C}(64)-\mathrm{H}(64 \mathrm{~A})$ & 0.9800 \\
\hline C(64)-H(64B) & 0.9800 \\
\hline C(64)-H(64C) & 0.9800 \\
\hline$C(73)-C(74)$ & $1.387(8)$ \\
\hline C(69)-H(69) & 0.9500 \\
\hline C(69)-C(70) & $1.383(9)$ \\
\hline$C(69)-C(68)$ & $1.385(9)$ \\
\hline $\mathrm{C}(8)-\mathrm{C}(9)$ & $1.542(8)$ \\
\hline$C(8)-C(10)$ & $1.531(9)$ \\
\hline $\mathrm{C}(8)-\mathrm{C}(11)$ & $1.512(8)$ \\
\hline$C(8)-C(7)$ & $1.532(8)$ \\
\hline $\mathrm{C}(62)-\mathrm{H}(62 \mathrm{~A})$ & 0.9800 \\
\hline $\mathrm{C}(62)-\mathrm{H}(62 \mathrm{~B})$ & 0.9800 \\
\hline $\mathrm{C}(62)-\mathrm{H}(62 \mathrm{C})$ & 0.9800 \\
\hline $\mathrm{C}(62)-\mathrm{C}(59)$ & $1.548(9)$ \\
\hline $\mathrm{C}(72)-\mathrm{H}(72)$ & 0.9500 \\
\hline $\mathrm{C}(72)-\mathrm{C}(67)$ & $1.391(9)$ \\
\hline C(9)-H(9A) & 0.9800 \\
\hline C(9)-H(9B) & 0.9800 \\
\hline C(9)-H(9C) & 0.9800 \\
\hline $\mathrm{C}(70)-\mathrm{H}(70)$ & 0.9500 \\
\hline C(14)-H(14) & 0.9500 \\
\hline$C(14)-C(15)$ & $1.391(9)$ \\
\hline
\end{tabular}




\begin{tabular}{|c|c|}
\hline $\mathrm{C}(59)-\mathrm{C}(58)$ & $1.521(8)$ \\
\hline $\mathrm{C}(59)-\mathrm{C}(60)$ & $1.528(8)$ \\
\hline $\mathrm{C}(59)-\mathrm{C}(61)$ & $1.544(8)$ \\
\hline $\mathrm{C}(50)-\mathrm{C}(49)$ & $1.422(9)$ \\
\hline$C(50)-C(51)$ & $1.544(8)$ \\
\hline $\mathrm{C}(24)-\mathrm{H}(24 \mathrm{~A})$ & 0.9800 \\
\hline $\mathrm{C}(24)-\mathrm{H}(24 \mathrm{~B})$ & 0.9800 \\
\hline $\mathrm{C}(24)-\mathrm{H}(24 \mathrm{C})$ & 0.9800 \\
\hline $\mathrm{C}(5)-\mathrm{N}(1)$ & $1.357(7)$ \\
\hline$C(5)-C(4)$ & $1.380(9)$ \\
\hline$C(5)-C(6)$ & $1.493(9)$ \\
\hline $\mathrm{C}(49)-\mathrm{C}(58)$ & $1.420(8)$ \\
\hline $\mathrm{C}(41)-\mathrm{H}(41)$ & 0.9500 \\
\hline $\mathrm{C}(41)-\mathrm{C}(42)$ & $1.383(9)$ \\
\hline $\mathrm{C}(41)-\mathrm{C}(40)$ & $1.389(10)$ \\
\hline$C(37)-C(42)$ & $1.391(8)$ \\
\hline C(37)-C(38) & $1.381(10)$ \\
\hline $\mathrm{C}(10)-\mathrm{H}(10 \mathrm{~A})$ & 0.9800 \\
\hline $\mathrm{C}(10)-\mathrm{H}(10 \mathrm{~B})$ & 0.9800 \\
\hline $\mathrm{C}(10)-\mathrm{H}(10 \mathrm{C})$ & 0.9800 \\
\hline $\mathrm{C}(76)-\mathrm{H}(76)$ & 0.9500 \\
\hline$C(76)-C(77)$ & $1.380(9)$ \\
\hline$C(76)-C(75)$ & $1.370(10)$ \\
\hline $\mathrm{C}(65)-\mathrm{H}(65 \mathrm{~A})$ & 0.9800 \\
\hline $\mathrm{C}(65)-\mathrm{H}(65 \mathrm{~B})$ & 0.9800 \\
\hline $\mathrm{C}(65)-\mathrm{H}(65 \mathrm{C})$ & 0.9800 \\
\hline $\mathrm{C}(12)-\mathrm{H}(12)$ & 0.9500 \\
\hline$C(12)-C(11)$ & $1.390(9)$ \\
\hline $\mathrm{C}(86)-\mathrm{D}(86)$ & 1.0000 \\
\hline C(74)-H(74) & 0.9500 \\
\hline$C(74)-C(75)$ & $1.401(9)$ \\
\hline C(84)-H(84) & 0.9500 \\
\hline $\mathrm{C}(84)-\mathrm{C}(83)$ & $1.389(9)$ \\
\hline $\mathrm{C}(80)-\mathrm{H}(80)$ & 0.9500 \\
\hline $\mathrm{C}(80)-\mathrm{C}(81)$ & $1.394(9)$ \\
\hline $\mathrm{C}(53)-\mathrm{H}(53 \mathrm{~A})$ & 0.9800 \\
\hline
\end{tabular}




\begin{tabular}{|c|c|}
\hline C(53)-H(53B) & 0.9800 \\
\hline $\mathrm{C}(53)-\mathrm{H}(53 \mathrm{C})$ & 0.9800 \\
\hline $\mathrm{C}(53)-\mathrm{C}(51)$ & $1.532(9)$ \\
\hline C(35)-H(35) & 0.9500 \\
\hline C(35)-C(34) & $1.369(9)$ \\
\hline$C(35)-C(36)$ & $1.390(9)$ \\
\hline $\mathrm{N}(1)-\mathrm{C}(1)$ & $1.355(8)$ \\
\hline $\mathrm{C}(42)-\mathrm{H}(42)$ & 0.9500 \\
\hline $\mathrm{C}(32)-\mathrm{H}(32)$ & 0.9500 \\
\hline $\mathrm{C}(32)-\mathrm{C}(31)$ & $1.390(9)$ \\
\hline C(32)-C(33) & $1.384(9)$ \\
\hline $\mathrm{C}(81)-\mathrm{H}(81)$ & 0.9500 \\
\hline C(77)-H(77) & 0.9500 \\
\hline $\mathrm{C}(30)-\mathrm{H}(30)$ & 0.9500 \\
\hline C(30)-C(25) & $1.397(8)$ \\
\hline C(30)-C(29) & $1.379(9)$ \\
\hline $\mathrm{C}(31)-\mathrm{C}(36)$ & $1.390(8)$ \\
\hline C(83)-H(83) & 0.9500 \\
\hline C(27)-H(27) & 0.9500 \\
\hline C(27)-C(26) & $1.387(9)$ \\
\hline C(26)-H(26) & 0.9500 \\
\hline$C(26)-C(25)$ & $1.390(9)$ \\
\hline C(43)-H(43) & 0.9500 \\
\hline C(43)-C(44) & $1.363(9)$ \\
\hline $\mathrm{C}(2)-\mathrm{H}(2)$ & 0.9500 \\
\hline$C(2)-C(1)$ & $1.362(9)$ \\
\hline$C(2)-C(3)$ & 1.391(9) \\
\hline$C(15)-C(16)$ & $1.408(8)$ \\
\hline$C(16)-C(11)$ & $1.412(8)$ \\
\hline C(38)-H(38) & 0.9500 \\
\hline C(38)-C(39) & $1.397(9)$ \\
\hline C(68)-H(68) & 0.9500 \\
\hline C(68)-C(67) & $1.400(9)$ \\
\hline $\mathrm{C}(75)-\mathrm{H}(75)$ & 0.9500 \\
\hline C(29)-H(29) & 0.9500 \\
\hline $\mathrm{C}(1)-\mathrm{H}(1)$ & 0.9500 \\
\hline
\end{tabular}




\begin{tabular}{|c|c|}
\hline $\mathrm{C}(85)-\mathrm{D}(85)$ & 1.0000 \\
\hline $\mathrm{C}(7)-\mathrm{H}(7 \mathrm{~A})$ & 0.9900 \\
\hline $\mathrm{C}(7)-\mathrm{H}(7 \mathrm{~B})$ & 0.9900 \\
\hline $\mathrm{C}(4)-\mathrm{H}(4)$ & 0.9500 \\
\hline$C(4)-C(3)$ & $1.358(9)$ \\
\hline$C(51)-C(54)$ & $1.541(9)$ \\
\hline C(34)-H(34) & 0.9500 \\
\hline$C(34)-C(33)$ & $1.391(9)$ \\
\hline $\mathrm{C}(54)-\mathrm{H}(54 \mathrm{~A})$ & 0.9800 \\
\hline $\mathrm{C}(54)-\mathrm{H}(54 \mathrm{~B})$ & 0.9800 \\
\hline $\mathrm{C}(54)-\mathrm{H}(54 \mathrm{C})$ & 0.9800 \\
\hline C(39)-H(39) & 0.9500 \\
\hline C(39)-C(40) & $1.373(10)$ \\
\hline $\mathrm{C}(40)-\mathrm{H}(40)$ & 0.9500 \\
\hline C(47)-C(48) & $1.504(9)$ \\
\hline C(47)-C(46) & $1.374(9)$ \\
\hline $\mathrm{C}(6)-\mathrm{H}(6 \mathrm{~A})$ & 0.9900 \\
\hline $\mathrm{C}(6)-\mathrm{H}(6 \mathrm{~B})$ & 0.9900 \\
\hline $\mathrm{C}(45)-\mathrm{H}(45)$ & 0.9500 \\
\hline $\mathrm{C}(45)-\mathrm{C}(46)$ & $1.378(9)$ \\
\hline C(45)-C(44) & $1.394(9)$ \\
\hline C(36)-H(36) & 0.9500 \\
\hline $\mathrm{C}(60)-\mathrm{H}(60 \mathrm{~A})$ & 0.9900 \\
\hline $\mathrm{C}(60)-\mathrm{H}(60 \mathrm{~B})$ & 0.9900 \\
\hline $\mathrm{C}(3)-\mathrm{H}(3)$ & 0.9500 \\
\hline $\mathrm{C}(61)-\mathrm{H}(61 \mathrm{~A})$ & 0.9800 \\
\hline $\mathrm{C}(61)-\mathrm{H}(61 \mathrm{~B})$ & 0.9800 \\
\hline $\mathrm{C}(61)-\mathrm{H}(61 \mathrm{C})$ & 0.9800 \\
\hline $\mathrm{C}(22)-\mathrm{H}(22 \mathrm{~A})$ & 0.9800 \\
\hline $\mathrm{C}(22)-\mathrm{H}(22 \mathrm{~B})$ & 0.9800 \\
\hline $\mathrm{C}(22)-\mathrm{H}(22 \mathrm{C})$ & 0.9800 \\
\hline $\mathrm{C}(48)-\mathrm{H}(48 \mathrm{~A})$ & 0.9900 \\
\hline $\mathrm{C}(48)-\mathrm{H}(48 \mathrm{~B})$ & 0.9900 \\
\hline $\mathrm{C}(46)-\mathrm{H}(46)$ & 0.9500 \\
\hline C(33)-H(33) & 0.9500 \\
\hline $\mathrm{C}(44)-\mathrm{H}(44)$ & 0.9500 \\
\hline
\end{tabular}




\begin{tabular}{|c|c|}
\hline $\mathrm{Cl}(2)-\mathrm{Ru}(1)-\mathrm{Cl}(1)$ & $89.59(5)$ \\
\hline $\mathrm{P}(1)-\mathrm{Ru}(1)-\mathrm{Cl}(1)$ & $107.46(6)$ \\
\hline $\mathrm{P}(1)-\mathrm{Ru}(1)-\mathrm{Cl}(2)$ & $91.37(6)$ \\
\hline $\mathrm{P}(2)-\mathrm{Ru}(1)-\mathrm{Cl}(1)$ & $154.64(6)$ \\
\hline $\mathrm{P}(2)-\mathrm{Ru}(1)-\mathrm{Cl}(2)$ & $97.66(6)$ \\
\hline $\mathrm{P}(2)-\mathrm{Ru}(1)-\mathrm{P}(1)$ & $96.68(6)$ \\
\hline $\mathrm{N}(1)-\mathrm{Ru}(1)-\mathrm{Cl}(1)$ & $86.93(14)$ \\
\hline $\mathrm{N}(1)-\mathrm{Ru}(1)-\mathrm{Cl}(2)$ & $174.76(15)$ \\
\hline $\mathrm{N}(1)-\mathrm{Ru}(1)-\mathrm{P}(1)$ & $93.41(15)$ \\
\hline $\mathrm{N}(1)-\mathrm{Ru}(1)-\mathrm{P}(2)$ & $83.92(15)$ \\
\hline $\mathrm{Cl}(3)-\mathrm{Ru}(2)-\mathrm{Cl}(4)$ & $89.03(6)$ \\
\hline $\mathrm{P}(4)-\mathrm{Ru}(2)-\mathrm{Cl}(3)$ & $98.85(6)$ \\
\hline $\mathrm{P}(4)-\mathrm{Ru}(2)-\mathrm{P}(3)$ & $97.08(6)$ \\
\hline $\mathrm{P}(4)-\mathrm{Ru}(2)-\mathrm{Cl}(4)$ & $154.62(7)$ \\
\hline $\mathrm{P}(3)-\mathrm{Ru}(2)-\mathrm{Cl}(3)$ & $91.12(6)$ \\
\hline $\mathrm{P}(3)-\mathrm{Ru}(2)-\mathrm{Cl}(4)$ & 106.91(6) \\
\hline $\mathrm{N}(2)-\mathrm{Ru}(2)-\mathrm{Cl}(3)$ & $173.94(14)$ \\
\hline $\mathrm{N}(2)-\mathrm{Ru}(2)-\mathrm{P}(4)$ & $84.11(14)$ \\
\hline $\mathrm{N}(2)-\mathrm{Ru}(2)-\mathrm{P}(3)$ & $93.76(15)$ \\
\hline $\mathrm{N}(2)-\mathrm{Ru}(2)-\mathrm{Cl}(4)$ & $86.13(14)$ \\
\hline $\mathrm{C}(37)-\mathrm{P}(1)-\mathrm{Ru}(1)$ & $117.0(2)$ \\
\hline $\mathrm{C}(31)-\mathrm{P}(1)-\mathrm{Ru}(1)$ & $118.9(2)$ \\
\hline $\mathrm{C}(31)-\mathrm{P}(1)-\mathrm{C}(37)$ & $99.3(3)$ \\
\hline$C(25)-P(1)-R u(1)$ & $112.9(2)$ \\
\hline $\mathrm{C}(25)-\mathrm{P}(1)-\mathrm{C}(37)$ & $103.0(3)$ \\
\hline $\mathrm{C}(25)-\mathrm{P}(1)-\mathrm{C}(31)$ & $103.5(3)$ \\
\hline $\mathrm{C}(49)-\mathrm{P}(4)-\mathrm{Ru}(2)$ & $122.4(2)$ \\
\hline C(49)-P(4)-C(48) & $101.9(3)$ \\
\hline $\mathrm{C}(60)-\mathrm{P}(4)-\mathrm{Ru}(2)$ & $129.1(2)$ \\
\hline $\mathrm{C}(60)-\mathrm{P}(4)-\mathrm{C}(49)$ & $91.9(3)$ \\
\hline $\mathrm{C}(60)-\mathrm{P}(4)-\mathrm{C}(48)$ & $105.8(3)$ \\
\hline $\mathrm{C}(48)-\mathrm{P}(4)-\mathrm{Ru}(2)$ & $102.4(2)$ \\
\hline$C(79)-P(3)-R u(2)$ & $118.4(2)$ \\
\hline C(79)-P(3)-C(73) & $99.3(3)$ \\
\hline $\mathrm{C}(73)-\mathrm{P}(3)-\mathrm{Ru}(2)$ & $117.5(2)$ \\
\hline
\end{tabular}




\begin{tabular}{|c|c|}
\hline $\mathrm{C}(67)-\mathrm{P}(3)-\mathrm{Ru}(2)$ & $112.4(2)$ \\
\hline $\mathrm{C}(67)-\mathrm{P}(3)-\mathrm{C}(79)$ & $104.6(3)$ \\
\hline $\mathrm{C}(67)-\mathrm{P}(3)-\mathrm{C}(73)$ & $102.5(3)$ \\
\hline $\mathrm{C}(16)-\mathrm{P}(2)-\mathrm{Ru}(1)$ & $122.6(2)$ \\
\hline $\mathrm{C}(16)-\mathrm{P}(2)-\mathrm{C}(6)$ & $102.3(3)$ \\
\hline $\mathrm{C}(7)-\mathrm{P}(2)-\mathrm{Ru}(1)$ & $128.8(2)$ \\
\hline$C(7)-P(2)-C(16)$ & $91.8(3)$ \\
\hline$C(7)-P(2)-C(6)$ & $106.0(3)$ \\
\hline $\mathrm{C}(6)-\mathrm{P}(2)-\mathrm{Ru}(1)$ & $102.1(2)$ \\
\hline $\mathrm{C}(56)-\mathrm{C}(57)-\mathrm{H}(57)$ & 119.4 \\
\hline $\mathrm{C}(58)-\mathrm{C}(57)-\mathrm{H}(57)$ & 119.4 \\
\hline$C(58)-C(57)-C(56)$ & 121.1(6) \\
\hline $\mathrm{C}(43)-\mathrm{N}(2)-\mathrm{Ru}(2)$ & $121.8(4)$ \\
\hline $\mathrm{C}(43)-\mathrm{N}(2)-\mathrm{C}(47)$ & $117.4(5)$ \\
\hline $\mathrm{C}(47)-\mathrm{N}(2)-\mathrm{Ru}(2)$ & $120.8(4)$ \\
\hline$H(20 A)-C(20)-H(20 B)$ & 109.5 \\
\hline$H(20 A)-C(20)-H(20 C)$ & 109.5 \\
\hline $\mathrm{H}(20 \mathrm{~B})-\mathrm{C}(20)-\mathrm{H}(20 \mathrm{C})$ & 109.5 \\
\hline $\mathrm{C}(17)-\mathrm{C}(20)-\mathrm{H}(20 \mathrm{~A})$ & 109.5 \\
\hline $\mathrm{C}(17)-\mathrm{C}(20)-\mathrm{H}(20 \mathrm{~B})$ & 109.5 \\
\hline $\mathrm{C}(17)-\mathrm{C}(20)-\mathrm{H}(20 \mathrm{C})$ & 109.5 \\
\hline$C(14)-C(13)-C(17)$ & $119.9(6)$ \\
\hline $\mathrm{C}(12)-\mathrm{C}(13)-\mathrm{C}(14)$ & $116.7(6)$ \\
\hline$C(12)-C(13)-C(17)$ & $123.4(6)$ \\
\hline$C(57)-C(56)-C(55)$ & $116.9(6)$ \\
\hline $\mathrm{C}(57)-\mathrm{C}(56)-\mathrm{C}(63)$ & $122.7(6)$ \\
\hline$C(55)-C(56)-C(63)$ & $120.3(6)$ \\
\hline $\mathrm{C}(56)-\mathrm{C}(55)-\mathrm{H}(55)$ & 117.4 \\
\hline$C(56)-C(55)-C(50)$ & $125.1(6)$ \\
\hline $\mathrm{C}(50)-\mathrm{C}(55)-\mathrm{H}(55)$ & 117.4 \\
\hline $\mathrm{H}(23 \mathrm{~A})-\mathrm{C}(23)-\mathrm{H}(23 \mathrm{~B})$ & 109.5 \\
\hline $\mathrm{H}(23 \mathrm{~A})-\mathrm{C}(23)-\mathrm{H}(23 \mathrm{C})$ & 109.5 \\
\hline $\mathrm{H}(23 \mathrm{~B})-\mathrm{C}(23)-\mathrm{H}(23 \mathrm{C})$ & 109.5 \\
\hline $\mathrm{C}(21)-\mathrm{C}(23)-\mathrm{H}(23 \mathrm{~A})$ & 109.5 \\
\hline $\mathrm{C}(21)-\mathrm{C}(23)-\mathrm{H}(23 \mathrm{~B})$ & 109.5 \\
\hline $\mathrm{C}(21)-\mathrm{C}(23)-\mathrm{H}(23 \mathrm{C})$ & 109.5 \\
\hline
\end{tabular}




\begin{tabular}{|c|c|}
\hline $\mathrm{H}(66 \mathrm{~A})-\mathrm{C}(66)-\mathrm{H}(66 \mathrm{~B})$ & 109.5 \\
\hline $\mathrm{H}(66 \mathrm{~A})-\mathrm{C}(66)-\mathrm{H}(66 \mathrm{C})$ & 109.5 \\
\hline $\mathrm{H}(66 \mathrm{~B})-\mathrm{C}(66)-\mathrm{H}(66 \mathrm{C})$ & 109.5 \\
\hline $\mathrm{C}(63)-\mathrm{C}(66)-\mathrm{H}(66 \mathrm{~A})$ & 109.5 \\
\hline $\mathrm{C}(63)-\mathrm{C}(66)-\mathrm{H}(66 \mathrm{~B})$ & 109.5 \\
\hline $\mathrm{C}(63)-\mathrm{C}(66)-\mathrm{H}(66 \mathrm{C})$ & 109.5 \\
\hline $\mathrm{C}(84)-\mathrm{C}(79)-\mathrm{P}(3)$ & $118.6(5)$ \\
\hline $\mathrm{C}(80)-\mathrm{C}(79)-\mathrm{P}(3)$ & $123.3(5)$ \\
\hline$C(80)-C(79)-C(84)$ & $118.1(6)$ \\
\hline$C(56)-C(63)-C(66)$ & $109.0(5)$ \\
\hline$C(56)-C(63)-C(64)$ & $112.7(6)$ \\
\hline$C(56)-C(63)-C(65)$ & $109.5(6)$ \\
\hline$C(64)-C(63)-C(66)$ & $107.9(6)$ \\
\hline$C(64)-C(63)-C(65)$ & $108.6(6)$ \\
\hline$C(65)-C(63)-C(66)$ & $109.1(6)$ \\
\hline $\mathrm{H}(18 \mathrm{~A})-\mathrm{C}(18)-\mathrm{H}(18 \mathrm{~B})$ & 109.5 \\
\hline $\mathrm{H}(18 \mathrm{~A})-\mathrm{C}(18)-\mathrm{H}(18 \mathrm{C})$ & 109.5 \\
\hline $\mathrm{H}(18 \mathrm{~B})-\mathrm{C}(18)-\mathrm{H}(18 \mathrm{C})$ & 109.5 \\
\hline $\mathrm{C}(17)-\mathrm{C}(18)-\mathrm{H}(18 \mathrm{~A})$ & 109.5 \\
\hline $\mathrm{C}(17)-\mathrm{C}(18)-\mathrm{H}(18 \mathrm{~B})$ & 109.5 \\
\hline $\mathrm{C}(17)-\mathrm{C}(18)-\mathrm{H}(18 \mathrm{C})$ & 109.5 \\
\hline $\mathrm{C}(73)-\mathrm{C}(78)-\mathrm{H}(78)$ & 119.6 \\
\hline$C(73)-C(78)-C(77)$ & $120.9(7)$ \\
\hline $\mathrm{C}(77)-\mathrm{C}(78)-\mathrm{H}(78)$ & 119.6 \\
\hline $\mathrm{H}(19 \mathrm{~A})-\mathrm{C}(19)-\mathrm{H}(19 \mathrm{~B})$ & 109.5 \\
\hline $\mathrm{H}(19 \mathrm{~A})-\mathrm{C}(19)-\mathrm{H}(19 \mathrm{C})$ & 109.5 \\
\hline $\mathrm{H}(19 \mathrm{~B})-\mathrm{C}(19)-\mathrm{H}(19 \mathrm{C})$ & 109.5 \\
\hline $\mathrm{C}(17)-\mathrm{C}(19)-\mathrm{H}(19 \mathrm{~A})$ & 109.5 \\
\hline $\mathrm{C}(17)-\mathrm{C}(19)-\mathrm{H}(19 \mathrm{~B})$ & 109.5 \\
\hline $\mathrm{C}(17)-\mathrm{C}(19)-\mathrm{H}(19 \mathrm{C})$ & 109.5 \\
\hline $\mathrm{C}(27)-\mathrm{C}(28)-\mathrm{H}(28)$ & 120.1 \\
\hline $\mathrm{C}(29)-\mathrm{C}(28)-\mathrm{H}(28)$ & 120.1 \\
\hline$C(29)-C(28)-C(27)$ & $119.8(7)$ \\
\hline$C(23)-C(21)-C(15)$ & $108.7(5)$ \\
\hline $\mathrm{C}(24)-\mathrm{C}(21)-\mathrm{C}(23)$ & $109.9(6)$ \\
\hline$C(24)-C(21)-C(15)$ & $109.3(5)$ \\
\hline
\end{tabular}




\begin{tabular}{|c|c|}
\hline$C(22)-C(21)-C(23)$ & $107.6(5)$ \\
\hline$C(22)-C(21)-C(24)$ & $107.4(6)$ \\
\hline$C(22)-C(21)-C(15)$ & $113.9(5)$ \\
\hline $\mathrm{C}(72)-\mathrm{C}(71)-\mathrm{H}(71)$ & 120.2 \\
\hline $\mathrm{C}(70)-\mathrm{C}(71)-\mathrm{H}(71)$ & 120.2 \\
\hline $\mathrm{C}(70)-\mathrm{C}(71)-\mathrm{C}(72)$ & $119.6(7)$ \\
\hline $\mathrm{H}(52 \mathrm{~A})-\mathrm{C}(52)-\mathrm{H}(52 \mathrm{~B})$ & 109.5 \\
\hline $\mathrm{H}(52 \mathrm{~A})-\mathrm{C}(52)-\mathrm{H}(52 \mathrm{C})$ & 109.5 \\
\hline $\mathrm{H}(52 \mathrm{~B})-\mathrm{C}(52)-\mathrm{H}(52 \mathrm{C})$ & 109.5 \\
\hline $\mathrm{C}(51)-\mathrm{C}(52)-\mathrm{H}(52 \mathrm{~A})$ & 109.5 \\
\hline $\mathrm{C}(51)-\mathrm{C}(52)-\mathrm{H}(52 \mathrm{~B})$ & 109.5 \\
\hline $\mathrm{C}(51)-\mathrm{C}(52)-\mathrm{H}(52 \mathrm{C})$ & 109.5 \\
\hline $\mathrm{C}(81)-\mathrm{C}(82)-\mathrm{H}(82)$ & 120.0 \\
\hline $\mathrm{C}(83)-\mathrm{C}(82)-\mathrm{H}(82)$ & 120.0 \\
\hline $\mathrm{C}(83)-\mathrm{C}(82)-\mathrm{C}(81)$ & $120.0(6)$ \\
\hline $\mathrm{C}(63)-\mathrm{C}(64)-\mathrm{H}(64 \mathrm{~A})$ & 109.5 \\
\hline $\mathrm{C}(63)-\mathrm{C}(64)-\mathrm{H}(64 \mathrm{~B})$ & 109.5 \\
\hline $\mathrm{C}(63)-\mathrm{C}(64)-\mathrm{H}(64 \mathrm{C})$ & 109.5 \\
\hline $\mathrm{H}(64 \mathrm{~A})-\mathrm{C}(64)-\mathrm{H}(64 \mathrm{~B})$ & 109.5 \\
\hline $\mathrm{H}(64 \mathrm{~A})-\mathrm{C}(64)-\mathrm{H}(64 \mathrm{C})$ & 109.5 \\
\hline $\mathrm{H}(64 \mathrm{~B})-\mathrm{C}(64)-\mathrm{H}(64 \mathrm{C})$ & 109.5 \\
\hline $\mathrm{C}(78)-\mathrm{C}(73)-\mathrm{P}(3)$ & $119.7(5)$ \\
\hline $\mathrm{C}(78)-\mathrm{C}(73)-\mathrm{C}(74)$ & 119.3(6) \\
\hline $\mathrm{C}(74)-\mathrm{C}(73)-\mathrm{P}(3)$ & $121.0(5)$ \\
\hline $\mathrm{C}(70)-\mathrm{C}(69)-\mathrm{H}(69)$ & 119.9 \\
\hline $\mathrm{C}(70)-\mathrm{C}(69)-\mathrm{C}(68)$ & $120.2(6)$ \\
\hline $\mathrm{C}(68)-\mathrm{C}(69)-\mathrm{H}(69)$ & 119.9 \\
\hline $\mathrm{C}(10)-\mathrm{C}(8)-\mathrm{C}(9)$ & $108.8(5)$ \\
\hline $\mathrm{C}(10)-\mathrm{C}(8)-\mathrm{C}(7)$ & $108.3(5)$ \\
\hline $\mathrm{C}(11)-\mathrm{C}(8)-\mathrm{C}(9)$ & $109.5(5)$ \\
\hline $\mathrm{C}(11)-\mathrm{C}(8)-\mathrm{C}(10)$ & 113.1(6) \\
\hline $\mathrm{C}(11)-\mathrm{C}(8)-\mathrm{C}(7)$ & $105.2(5)$ \\
\hline $\mathrm{C}(7)-\mathrm{C}(8)-\mathrm{C}(9)$ & $111.9(5)$ \\
\hline $\mathrm{H}(62 \mathrm{~A})-\mathrm{C}(62)-\mathrm{H}(62 \mathrm{~B})$ & 109.5 \\
\hline $\mathrm{H}(62 \mathrm{~A})-\mathrm{C}(62)-\mathrm{H}(62 \mathrm{C})$ & 109.5 \\
\hline $\mathrm{H}(62 \mathrm{~B})-\mathrm{C}(62)-\mathrm{H}(62 \mathrm{C})$ & 109.5 \\
\hline
\end{tabular}




\begin{tabular}{|c|c|}
\hline $\mathrm{C}(59)-\mathrm{C}(62)-\mathrm{H}(62 \mathrm{~A})$ & 109.5 \\
\hline $\mathrm{C}(59)-\mathrm{C}(62)-\mathrm{H}(62 \mathrm{~B})$ & 109.5 \\
\hline $\mathrm{C}(59)-\mathrm{C}(62)-\mathrm{H}(62 \mathrm{C})$ & 109.5 \\
\hline $\mathrm{C}(71)-\mathrm{C}(72)-\mathrm{H}(72)$ & 119.3 \\
\hline $\mathrm{C}(71)-\mathrm{C}(72)-\mathrm{C}(67)$ & $121.4(6)$ \\
\hline $\mathrm{C}(67)-\mathrm{C}(72)-\mathrm{H}(72)$ & 119.3 \\
\hline $\mathrm{C}(8)-\mathrm{C}(9)-\mathrm{H}(9 \mathrm{~A})$ & 109.5 \\
\hline $\mathrm{C}(8)-\mathrm{C}(9)-\mathrm{H}(9 \mathrm{~B})$ & 109.5 \\
\hline $\mathrm{C}(8)-\mathrm{C}(9)-\mathrm{H}(9 \mathrm{C})$ & 109.5 \\
\hline $\mathrm{H}(9 \mathrm{~A})-\mathrm{C}(9)-\mathrm{H}(9 \mathrm{~B})$ & 109.5 \\
\hline $\mathrm{H}(9 \mathrm{~A})-\mathrm{C}(9)-\mathrm{H}(9 \mathrm{C})$ & 109.5 \\
\hline H(9B)-C(9)-H(9C) & 109.5 \\
\hline $\mathrm{C}(71)-\mathrm{C}(70)-\mathrm{H}(70)$ & 120.0 \\
\hline $\mathrm{C}(69)-\mathrm{C}(70)-\mathrm{C}(71)$ & $120.0(6)$ \\
\hline $\mathrm{C}(69)-\mathrm{C}(70)-\mathrm{H}(70)$ & 120.0 \\
\hline $\mathrm{C}(13)-\mathrm{C}(14)-\mathrm{H}(14)$ & 117.8 \\
\hline$C(15)-C(14)-C(13)$ & $124.3(6)$ \\
\hline $\mathrm{C}(15)-\mathrm{C}(14)-\mathrm{H}(14)$ & 117.8 \\
\hline$C(58)-C(59)-C(62)$ & $112.3(5)$ \\
\hline$C(58)-C(59)-C(60)$ & $105.5(5)$ \\
\hline $\mathrm{C}(58)-\mathrm{C}(59)-\mathrm{C}(61)$ & $109.3(5)$ \\
\hline $\mathrm{C}(60)-\mathrm{C}(59)-\mathrm{C}(62)$ & $109.0(5)$ \\
\hline $\mathrm{C}(60)-\mathrm{C}(59)-\mathrm{C}(61)$ & $112.0(5)$ \\
\hline $\mathrm{C}(61)-\mathrm{C}(59)-\mathrm{C}(62)$ & $108.7(5)$ \\
\hline $\mathrm{C}(13)-\mathrm{C}(17)-\mathrm{C}(20)$ & $109.9(5)$ \\
\hline $\mathrm{C}(13)-\mathrm{C}(17)-\mathrm{C}(18)$ & $111.9(5)$ \\
\hline $\mathrm{C}(13)-\mathrm{C}(17)-\mathrm{C}(19)$ & $108.7(6)$ \\
\hline $\mathrm{C}(18)-\mathrm{C}(17)-\mathrm{C}(20)$ & $108.0(6)$ \\
\hline$C(19)-C(17)-C(20)$ & $110.3(6)$ \\
\hline $\mathrm{C}(19)-\mathrm{C}(17)-\mathrm{C}(18)$ & $108.0(5)$ \\
\hline$C(55)-C(50)-C(49)$ & $116.7(6)$ \\
\hline $\mathrm{C}(55)-\mathrm{C}(50)-\mathrm{C}(51)$ & $115.6(6)$ \\
\hline $\mathrm{C}(49)-\mathrm{C}(50)-\mathrm{C}(51)$ & $127.6(6)$ \\
\hline $\mathrm{C}(21)-\mathrm{C}(24)-\mathrm{H}(24 \mathrm{~A})$ & 109.5 \\
\hline $\mathrm{C}(21)-\mathrm{C}(24)-\mathrm{H}(24 \mathrm{~B})$ & 109.5 \\
\hline $\mathrm{C}(21)-\mathrm{C}(24)-\mathrm{H}(24 \mathrm{C})$ & 109.5 \\
\hline
\end{tabular}




\begin{tabular}{|c|c|}
\hline $\mathrm{H}(24 \mathrm{~A})-\mathrm{C}(24)-\mathrm{H}(24 \mathrm{~B})$ & 109.5 \\
\hline $\mathrm{H}(24 \mathrm{~A})-\mathrm{C}(24)-\mathrm{H}(24 \mathrm{C})$ & 109.5 \\
\hline $\mathrm{H}(24 \mathrm{~B})-\mathrm{C}(24)-\mathrm{H}(24 \mathrm{C})$ & 109.5 \\
\hline $\mathrm{N}(1)-\mathrm{C}(5)-\mathrm{C}(4)$ & $122.2(6)$ \\
\hline $\mathrm{N}(1)-\mathrm{C}(5)-\mathrm{C}(6)$ & $118.0(6)$ \\
\hline$C(4)-C(5)-C(6)$ & $119.7(6)$ \\
\hline $\mathrm{C}(50)-\mathrm{C}(49)-\mathrm{P}(4)$ & $132.1(5)$ \\
\hline $\mathrm{C}(58)-\mathrm{C}(49)-\mathrm{P}(4)$ & $109.0(5)$ \\
\hline $\mathrm{C}(58)-\mathrm{C}(49)-\mathrm{C}(50)$ & $118.9(6)$ \\
\hline $\mathrm{C}(42)-\mathrm{C}(41)-\mathrm{H}(41)$ & 120.0 \\
\hline$C(42)-C(41)-C(40)$ & $119.9(7)$ \\
\hline $\mathrm{C}(40)-\mathrm{C}(41)-\mathrm{H}(41)$ & 120.0 \\
\hline $\mathrm{C}(42)-\mathrm{C}(37)-\mathrm{P}(1)$ & $121.2(5)$ \\
\hline $\mathrm{C}(38)-\mathrm{C}(37)-\mathrm{P}(1)$ & $120.4(5)$ \\
\hline $\mathrm{C}(38)-\mathrm{C}(37)-\mathrm{C}(42)$ & $118.2(6)$ \\
\hline $\mathrm{C}(8)-\mathrm{C}(10)-\mathrm{H}(10 \mathrm{~A})$ & 109.5 \\
\hline $\mathrm{C}(8)-\mathrm{C}(10)-\mathrm{H}(10 \mathrm{~B})$ & 109.5 \\
\hline $\mathrm{C}(8)-\mathrm{C}(10)-\mathrm{H}(10 \mathrm{C})$ & 109.5 \\
\hline $\mathrm{H}(10 \mathrm{~A})-\mathrm{C}(10)-\mathrm{H}(10 \mathrm{~B})$ & 109.5 \\
\hline $\mathrm{H}(10 \mathrm{~A})-\mathrm{C}(10)-\mathrm{H}(10 \mathrm{C})$ & 109.5 \\
\hline $\mathrm{H}(10 \mathrm{~B})-\mathrm{C}(10)-\mathrm{H}(10 \mathrm{C})$ & 109.5 \\
\hline $\mathrm{C}(57)-\mathrm{C}(58)-\mathrm{C}(59)$ & $121.2(6)$ \\
\hline $\mathrm{C}(57)-\mathrm{C}(58)-\mathrm{C}(49)$ & 121.2(6) \\
\hline $\mathrm{C}(49)-\mathrm{C}(58)-\mathrm{C}(59)$ & $117.5(6)$ \\
\hline $\mathrm{C}(77)-\mathrm{C}(76)-\mathrm{H}(76)$ & 120.2 \\
\hline $\mathrm{C}(75)-\mathrm{C}(76)-\mathrm{H}(76)$ & 120.2 \\
\hline$C(75)-C(76)-C(77)$ & $119.6(7)$ \\
\hline $\mathrm{C}(63)-\mathrm{C}(65)-\mathrm{H}(65 \mathrm{~A})$ & 109.5 \\
\hline $\mathrm{C}(63)-\mathrm{C}(65)-\mathrm{H}(65 \mathrm{~B})$ & 109.5 \\
\hline $\mathrm{C}(63)-\mathrm{C}(65)-\mathrm{H}(65 \mathrm{C})$ & 109.5 \\
\hline $\mathrm{H}(65 \mathrm{~A})-\mathrm{C}(65)-\mathrm{H}(65 \mathrm{~B})$ & 109.5 \\
\hline $\mathrm{H}(65 \mathrm{~A})-\mathrm{C}(65)-\mathrm{H}(65 \mathrm{C})$ & 109.5 \\
\hline $\mathrm{H}(65 \mathrm{~B})-\mathrm{C}(65)-\mathrm{H}(65 \mathrm{C})$ & 109.5 \\
\hline $\mathrm{C}(13)-\mathrm{C}(12)-\mathrm{H}(12)$ & 119.1 \\
\hline$C(13)-C(12)-C(11)$ & $121.7(6)$ \\
\hline $\mathrm{C}(11)-\mathrm{C}(12)-\mathrm{H}(12)$ & 119.1 \\
\hline
\end{tabular}




\begin{tabular}{|c|c|}
\hline $\mathrm{Cl}(5)-\mathrm{C}(86)-\mathrm{D}(86)$ & 108.8 \\
\hline $\mathrm{Cl}(6)-\mathrm{C}(86)-\mathrm{Cl}(5)$ & $109.7(4)$ \\
\hline $\mathrm{Cl}(6)-\mathrm{C}(86)-\mathrm{D}(86)$ & 108.8 \\
\hline $\mathrm{Cl}(7)-\mathrm{C}(86)-\mathrm{Cl}(5)$ & $109.8(4)$ \\
\hline $\mathrm{Cl}(7)-\mathrm{C}(86)-\mathrm{Cl}(6)$ & $111.0(4)$ \\
\hline $\mathrm{Cl}(7)-\mathrm{C}(86)-\mathrm{D}(86)$ & 108.8 \\
\hline $\mathrm{C}(73)-\mathrm{C}(74)-\mathrm{H}(74)$ & 120.3 \\
\hline$C(73)-C(74)-C(75)$ & $119.4(7)$ \\
\hline $\mathrm{C}(75)-\mathrm{C}(74)-\mathrm{H}(74)$ & 120.3 \\
\hline $\mathrm{C}(79)-\mathrm{C}(84)-\mathrm{H}(84)$ & 119.7 \\
\hline $\mathrm{C}(83)-\mathrm{C}(84)-\mathrm{C}(79)$ & $120.6(6)$ \\
\hline $\mathrm{C}(83)-\mathrm{C}(84)-\mathrm{H}(84)$ & 119.7 \\
\hline $\mathrm{C}(79)-\mathrm{C}(80)-\mathrm{H}(80)$ & 119.3 \\
\hline $\mathrm{C}(79)-\mathrm{C}(80)-\mathrm{C}(81)$ & $121.3(7)$ \\
\hline $\mathrm{C}(81)-\mathrm{C}(80)-\mathrm{H}(80)$ & 119.3 \\
\hline $\mathrm{H}(53 \mathrm{~A})-\mathrm{C}(53)-\mathrm{H}(53 \mathrm{~B})$ & 109.5 \\
\hline $\mathrm{H}(53 \mathrm{~A})-\mathrm{C}(53)-\mathrm{H}(53 \mathrm{C})$ & 109.5 \\
\hline $\mathrm{H}(53 \mathrm{~B})-\mathrm{C}(53)-\mathrm{H}(53 \mathrm{C})$ & 109.5 \\
\hline $\mathrm{C}(51)-\mathrm{C}(53)-\mathrm{H}(53 \mathrm{~A})$ & 109.5 \\
\hline $\mathrm{C}(51)-\mathrm{C}(53)-\mathrm{H}(53 \mathrm{~B})$ & 109.5 \\
\hline $\mathrm{C}(51)-\mathrm{C}(53)-\mathrm{H}(53 \mathrm{C})$ & 109.5 \\
\hline $\mathrm{C}(34)-\mathrm{C}(35)-\mathrm{H}(35)$ & 120.0 \\
\hline $\mathrm{C}(34)-\mathrm{C}(35)-\mathrm{C}(36)$ & $120.0(6)$ \\
\hline $\mathrm{C}(36)-\mathrm{C}(35)-\mathrm{H}(35)$ & 120.0 \\
\hline $\mathrm{C}(5)-\mathrm{N}(1)-\mathrm{Ru}(1)$ & $121.6(4)$ \\
\hline $\mathrm{C}(1)-\mathrm{N}(1)-\mathrm{Ru}(1)$ & $121.5(4)$ \\
\hline $\mathrm{C}(1)-\mathrm{N}(1)-\mathrm{C}(5)$ & $116.8(6)$ \\
\hline $\mathrm{C}(41)-\mathrm{C}(42)-\mathrm{C}(37)$ & $121.0(7)$ \\
\hline $\mathrm{C}(41)-\mathrm{C}(42)-\mathrm{H}(42)$ & 119.5 \\
\hline $\mathrm{C}(37)-\mathrm{C}(42)-\mathrm{H}(42)$ & 119.5 \\
\hline $\mathrm{C}(31)-\mathrm{C}(32)-\mathrm{H}(32)$ & 119.3 \\
\hline $\mathrm{C}(33)-\mathrm{C}(32)-\mathrm{H}(32)$ & 119.3 \\
\hline $\mathrm{C}(33)-\mathrm{C}(32)-\mathrm{C}(31)$ & $121.4(6)$ \\
\hline $\mathrm{C}(82)-\mathrm{C}(81)-\mathrm{C}(80)$ & $119.5(6)$ \\
\hline $\mathrm{C}(82)-\mathrm{C}(81)-\mathrm{H}(81)$ & 120.2 \\
\hline $\mathrm{C}(80)-\mathrm{C}(81)-\mathrm{H}(81)$ & 120.2 \\
\hline
\end{tabular}




\begin{tabular}{|c|c|}
\hline $\mathrm{C}(78)-\mathrm{C}(77)-\mathrm{H}(77)$ & 120.1 \\
\hline$C(76)-C(77)-C(78)$ & $119.8(8)$ \\
\hline $\mathrm{C}(76)-\mathrm{C}(77)-\mathrm{H}(77)$ & 120.1 \\
\hline $\mathrm{C}(25)-\mathrm{C}(30)-\mathrm{H}(30)$ & 119.5 \\
\hline $\mathrm{C}(29)-\mathrm{C}(30)-\mathrm{H}(30)$ & 119.5 \\
\hline$C(29)-C(30)-C(25)$ & $121.0(6)$ \\
\hline $\mathrm{C}(32)-\mathrm{C}(31)-\mathrm{P}(1)$ & $119.3(5)$ \\
\hline $\mathrm{C}(36)-\mathrm{C}(31)-\mathrm{P}(1)$ & $122.9(5)$ \\
\hline $\mathrm{C}(36)-\mathrm{C}(31)-\mathrm{C}(32)$ & $117.8(6)$ \\
\hline $\mathrm{C}(82)-\mathrm{C}(83)-\mathrm{C}(84)$ & $120.4(7)$ \\
\hline $\mathrm{C}(82)-\mathrm{C}(83)-\mathrm{H}(83)$ & 119.8 \\
\hline $\mathrm{C}(84)-\mathrm{C}(83)-\mathrm{H}(83)$ & 119.8 \\
\hline $\mathrm{C}(28)-\mathrm{C}(27)-\mathrm{H}(27)$ & 120.2 \\
\hline $\mathrm{C}(26)-\mathrm{C}(27)-\mathrm{C}(28)$ & $119.7(7)$ \\
\hline $\mathrm{C}(26)-\mathrm{C}(27)-\mathrm{H}(27)$ & 120.2 \\
\hline $\mathrm{C}(27)-\mathrm{C}(26)-\mathrm{H}(26)$ & 119.5 \\
\hline$C(27)-C(26)-C(25)$ & $121.0(6)$ \\
\hline$C(25)-C(26)-H(26)$ & 119.5 \\
\hline $\mathrm{N}(2)-\mathrm{C}(43)-\mathrm{H}(43)$ & 118.4 \\
\hline $\mathrm{N}(2)-\mathrm{C}(43)-\mathrm{C}(44)$ & $123.2(6)$ \\
\hline $\mathrm{C}(44)-\mathrm{C}(43)-\mathrm{H}(43)$ & 118.4 \\
\hline $\mathrm{C}(1)-\mathrm{C}(2)-\mathrm{H}(2)$ & 120.4 \\
\hline$C(1)-C(2)-C(3)$ & $119.3(7)$ \\
\hline $\mathrm{C}(3)-\mathrm{C}(2)-\mathrm{H}(2)$ & 120.4 \\
\hline$C(14)-C(15)-C(21)$ & $114.6(6)$ \\
\hline $\mathrm{C}(14)-\mathrm{C}(15)-\mathrm{C}(16)$ & $117.7(6)$ \\
\hline$C(16)-C(15)-C(21)$ & $127.6(6)$ \\
\hline $\mathrm{C}(15)-\mathrm{C}(16)-\mathrm{P}(2)$ & $132.3(5)$ \\
\hline$C(15)-C(16)-C(11)$ & $118.9(6)$ \\
\hline $\mathrm{C}(11)-\mathrm{C}(16)-\mathrm{P}(2)$ & $108.5(4)$ \\
\hline $\mathrm{C}(37)-\mathrm{C}(38)-\mathrm{H}(38)$ & 119.4 \\
\hline $\mathrm{C}(37)-\mathrm{C}(38)-\mathrm{C}(39)$ & 121.3(7) \\
\hline $\mathrm{C}(39)-\mathrm{C}(38)-\mathrm{H}(38)$ & 119.4 \\
\hline $\mathrm{C}(30)-\mathrm{C}(25)-\mathrm{P}(1)$ & $119.2(5)$ \\
\hline $\mathrm{C}(26)-\mathrm{C}(25)-\mathrm{P}(1)$ & $122.3(5)$ \\
\hline$C(26)-C(25)-C(30)$ & $118.2(6)$ \\
\hline
\end{tabular}




\begin{tabular}{|c|c|}
\hline $\mathrm{C}(69)-\mathrm{C}(68)-\mathrm{H}(68)$ & 119.6 \\
\hline$C(69)-C(68)-C(67)$ & $120.8(6)$ \\
\hline $\mathrm{C}(67)-\mathrm{C}(68)-\mathrm{H}(68)$ & 119.6 \\
\hline$C(76)-C(75)-C(74)$ & $121.1(7)$ \\
\hline $\mathrm{C}(76)-\mathrm{C}(75)-\mathrm{H}(75)$ & 119.5 \\
\hline $\mathrm{C}(74)-\mathrm{C}(75)-\mathrm{H}(75)$ & 119.5 \\
\hline $\mathrm{C}(12)-\mathrm{C}(11)-\mathrm{C}(8)$ & $121.0(5)$ \\
\hline$C(12)-C(11)-C(16)$ & $120.6(6)$ \\
\hline$C(16)-C(11)-C(8)$ & $118.2(5)$ \\
\hline $\mathrm{C}(28)-\mathrm{C}(29)-\mathrm{C}(30)$ & $120.2(7)$ \\
\hline $\mathrm{C}(28)-\mathrm{C}(29)-\mathrm{H}(29)$ & 119.9 \\
\hline $\mathrm{C}(30)-\mathrm{C}(29)-\mathrm{H}(29)$ & 119.9 \\
\hline $\mathrm{N}(1)-\mathrm{C}(1)-\mathrm{C}(2)$ & $123.1(6)$ \\
\hline $\mathrm{N}(1)-\mathrm{C}(1)-\mathrm{H}(1)$ & 118.5 \\
\hline $\mathrm{C}(2)-\mathrm{C}(1)-\mathrm{H}(1)$ & 118.5 \\
\hline $\mathrm{Cl}(9)-\mathrm{C}(85)-\mathrm{Cl}(10)$ & $109.7(4)$ \\
\hline $\mathrm{Cl}(9)-\mathrm{C}(85)-\mathrm{D}(85)$ & 108.9 \\
\hline $\mathrm{Cl}(10)-\mathrm{C}(85)-\mathrm{D}(85)$ & 108.9 \\
\hline $\mathrm{Cl}(8)-\mathrm{C}(85)-\mathrm{Cl}(9)$ & $110.6(4)$ \\
\hline $\mathrm{Cl}(8)-\mathrm{C}(85)-\mathrm{Cl}(10)$ & $109.9(4)$ \\
\hline $\mathrm{Cl}(8)-\mathrm{C}(85)-\mathrm{D}(85)$ & 108.9 \\
\hline $\mathrm{P}(2)-\mathrm{C}(7)-\mathrm{H}(7 \mathrm{~A})$ & 109.9 \\
\hline $\mathrm{P}(2)-\mathrm{C}(7)-\mathrm{H}(7 \mathrm{~B})$ & 109.9 \\
\hline $\mathrm{C}(8)-\mathrm{C}(7)-\mathrm{P}(2)$ & 109.1(4) \\
\hline $\mathrm{C}(8)-\mathrm{C}(7)-\mathrm{H}(7 \mathrm{~A})$ & 109.9 \\
\hline $\mathrm{C}(8)-\mathrm{C}(7)-\mathrm{H}(7 \mathrm{~B})$ & 109.9 \\
\hline $\mathrm{H}(7 \mathrm{~A})-\mathrm{C}(7)-\mathrm{H}(7 \mathrm{~B})$ & 108.3 \\
\hline $\mathrm{C}(5)-\mathrm{C}(4)-\mathrm{H}(4)$ & 120.0 \\
\hline$C(3)-C(4)-C(5)$ & $120.0(6)$ \\
\hline $\mathrm{C}(3)-\mathrm{C}(4)-\mathrm{H}(4)$ & 120.0 \\
\hline$C(52)-C(51)-C(50)$ & $114.9(5)$ \\
\hline$C(53)-C(51)-C(52)$ & $107.4(5)$ \\
\hline$C(53)-C(51)-C(50)$ & $109.5(6)$ \\
\hline$C(53)-C(51)-C(54)$ & $109.4(6)$ \\
\hline $\mathrm{C}(54)-\mathrm{C}(51)-\mathrm{C}(52)$ & $106.0(6)$ \\
\hline $\mathrm{C}(54)-\mathrm{C}(51)-\mathrm{C}(50)$ & $109.4(5)$ \\
\hline
\end{tabular}




\begin{tabular}{|c|c|}
\hline $\mathrm{C}(35)-\mathrm{C}(34)-\mathrm{H}(34)$ & 120.0 \\
\hline$C(35)-C(34)-C(33)$ & $120.1(6)$ \\
\hline $\mathrm{C}(33)-\mathrm{C}(34)-\mathrm{H}(34)$ & 120.0 \\
\hline $\mathrm{C}(51)-\mathrm{C}(54)-\mathrm{H}(54 \mathrm{~A})$ & 109.5 \\
\hline $\mathrm{C}(51)-\mathrm{C}(54)-\mathrm{H}(54 \mathrm{~B})$ & 109.5 \\
\hline $\mathrm{C}(51)-\mathrm{C}(54)-\mathrm{H}(54 \mathrm{C})$ & 109.5 \\
\hline $\mathrm{H}(54 \mathrm{~A})-\mathrm{C}(54)-\mathrm{H}(54 \mathrm{~B})$ & 109.5 \\
\hline $\mathrm{H}(54 \mathrm{~A})-\mathrm{C}(54)-\mathrm{H}(54 \mathrm{C})$ & 109.5 \\
\hline $\mathrm{H}(54 \mathrm{~B})-\mathrm{C}(54)-\mathrm{H}(54 \mathrm{C})$ & 109.5 \\
\hline $\mathrm{C}(38)-\mathrm{C}(39)-\mathrm{H}(39)$ & 120.2 \\
\hline $\mathrm{C}(40)-\mathrm{C}(39)-\mathrm{C}(38)$ & $119.6(8)$ \\
\hline $\mathrm{C}(40)-\mathrm{C}(39)-\mathrm{H}(39)$ & 120.2 \\
\hline $\mathrm{C}(41)-\mathrm{C}(40)-\mathrm{H}(40)$ & 120.0 \\
\hline$C(39)-C(40)-C(41)$ & $120.0(6)$ \\
\hline $\mathrm{C}(39)-\mathrm{C}(40)-\mathrm{H}(40)$ & 120.0 \\
\hline $\mathrm{N}(2)-\mathrm{C}(47)-\mathrm{C}(48)$ & $118.6(6)$ \\
\hline $\mathrm{N}(2)-\mathrm{C}(47)-\mathrm{C}(46)$ & $121.5(6)$ \\
\hline$C(46)-C(47)-C(48)$ & $119.8(6)$ \\
\hline $\mathrm{P}(2)-\mathrm{C}(6)-\mathrm{H}(6 \mathrm{~A})$ & 109.0 \\
\hline $\mathrm{P}(2)-\mathrm{C}(6)-\mathrm{H}(6 \mathrm{~B})$ & 109.0 \\
\hline $\mathrm{C}(5)-\mathrm{C}(6)-\mathrm{P}(2)$ & $112.9(4)$ \\
\hline $\mathrm{C}(5)-\mathrm{C}(6)-\mathrm{H}(6 \mathrm{~A})$ & 109.0 \\
\hline $\mathrm{C}(5)-\mathrm{C}(6)-\mathrm{H}(6 \mathrm{~B})$ & 109.0 \\
\hline $\mathrm{H}(6 \mathrm{~A})-\mathrm{C}(6)-\mathrm{H}(6 \mathrm{~B})$ & 107.8 \\
\hline $\mathrm{C}(46)-\mathrm{C}(45)-\mathrm{H}(45)$ & 121.1 \\
\hline $\mathrm{C}(46)-\mathrm{C}(45)-\mathrm{C}(44)$ & $117.7(7)$ \\
\hline $\mathrm{C}(44)-\mathrm{C}(45)-\mathrm{H}(45)$ & 121.1 \\
\hline $\mathrm{C}(35)-\mathrm{C}(36)-\mathrm{H}(36)$ & 119.4 \\
\hline$C(31)-C(36)-C(35)$ & $121.2(7)$ \\
\hline $\mathrm{C}(31)-\mathrm{C}(36)-\mathrm{H}(36)$ & 119.4 \\
\hline$P(4)-C(60)-H(60 A)$ & 109.7 \\
\hline $\mathrm{P}(4)-\mathrm{C}(60)-\mathrm{H}(60 \mathrm{~B})$ & 109.7 \\
\hline $\mathrm{C}(59)-\mathrm{C}(60)-\mathrm{P}(4)$ & $109.9(4)$ \\
\hline $\mathrm{C}(59)-\mathrm{C}(60)-\mathrm{H}(60 \mathrm{~A})$ & 109.7 \\
\hline $\mathrm{C}(59)-\mathrm{C}(60)-\mathrm{H}(60 \mathrm{~B})$ & 109.7 \\
\hline $\mathrm{H}(60 \mathrm{~A})-\mathrm{C}(60)-\mathrm{H}(60 \mathrm{~B})$ & 108.2 \\
\hline
\end{tabular}




$\begin{array}{ll}\mathrm{C}(2)-\mathrm{C}(3)-\mathrm{H}(3) & 120.8 \\ \mathrm{C}(4)-\mathrm{C}(3)-\mathrm{C}(2) & 118.5(7) \\ \mathrm{C}(4)-\mathrm{C}(3)-\mathrm{H}(3) & 120.8 \\ \mathrm{C}(72)-\mathrm{C}(67)-\mathrm{P}(3) & 122.7(5) \\ \mathrm{C}(72)-\mathrm{C}(67)-\mathrm{C}(68) & 117.9(6) \\ \mathrm{C}(68)-\mathrm{C}(67)-\mathrm{P}(3) & 118.9(5) \\ \mathrm{C}(59)-\mathrm{C}(61)-\mathrm{H}(61 \mathrm{~A}) & 109.5 \\ \mathrm{C}(59)-\mathrm{C}(61)-\mathrm{H}(61 \mathrm{~B}) & 109.5 \\ \mathrm{C}(59)-\mathrm{C}(61)-\mathrm{H}(61 \mathrm{C}) & 109.5 \\ \mathrm{H}(61 \mathrm{~A})-\mathrm{C}(61)-\mathrm{H}(61 \mathrm{~B}) & 109.5 \\ \mathrm{H}(61 \mathrm{~A})-\mathrm{C}(61)-\mathrm{H}(61 \mathrm{C}) & 109.5 \\ \mathrm{H}(61 \mathrm{~B})-\mathrm{C}(61)-\mathrm{H}(61 \mathrm{C}) & 109.5 \\ \mathrm{C}(21)-\mathrm{C}(22)-\mathrm{H}(22 \mathrm{~A}) & 109.5 \\ \mathrm{C}(21)-\mathrm{C}(22)-\mathrm{H}(22 \mathrm{~B}) & 109.5 \\ \mathrm{C}(21)-\mathrm{C}(22)-\mathrm{H}(22 \mathrm{C}) & 109.5 \\ \mathrm{H}(22 \mathrm{~A})-\mathrm{C}(22)-\mathrm{H}(22 \mathrm{~B}) & 109.5 \\ \mathrm{H}(22 \mathrm{~A})-\mathrm{C}(22)-\mathrm{H}(22 \mathrm{C}) & 109.5 \\ \mathrm{H}(22 \mathrm{~B})-\mathrm{C}(22)-\mathrm{H}(22 \mathrm{C}) & 109.5 \\ \mathrm{P}(4)-\mathrm{C}(48)-\mathrm{H}(48 \mathrm{~A}) & 109.1 \\ \mathrm{P}(4)-\mathrm{C}(48)-\mathrm{H}(48 \mathrm{~B}) & 109.1 \\ \mathrm{C}(47)-\mathrm{C}(48)-\mathrm{P}(4) & 112.6(4) \\ \mathrm{C}(47)-\mathrm{C}(48)-\mathrm{H}(48 \mathrm{~A}) & 109.1 \\ \mathrm{C}(47)-\mathrm{C}(48)-\mathrm{H}(48 \mathrm{~B}) & 109.1 \\ \mathrm{H}(48 \mathrm{~A})-\mathrm{C}(48)-\mathrm{H}(48 \mathrm{~B}) & 107.8 \\ \mathrm{C}(47)-\mathrm{C}(46)-\mathrm{C}(45) & 120.6(6) \\ \mathrm{C}(47)-\mathrm{C}(46)-\mathrm{H}(46) & 119.7 \\ \mathrm{C}(45)-\mathrm{C}(46)-\mathrm{H}(46) & 119.7 \\ \mathrm{C}(32)-\mathrm{C}(33)-\mathrm{C}(34) & 119.5(7) \\ \mathrm{C}(32)-\mathrm{C}(33)-\mathrm{H}(33) & 120.2 \\ \mathrm{C}(34)-\mathrm{C}(33)-\mathrm{H}(33) & 120.2 \\ \mathrm{C}(43)-\mathrm{C}(44)-\mathrm{C}(45) & 119.4(6) \\ \mathrm{C}(43)-\mathrm{C}(44)-\mathrm{H}(44) & 120.3 \\ & 120.3 \\ & \end{array}$

Symmetry transformations used to generate equivalent atoms: 
Table S34. Anisotropic displacement parameters $\left(\AA^{2} \times 10^{3}\right)$ for 5a. The anisotropic displacement factor exponent takes the form: $-2 \pi^{2}\left[h^{2} a^{* 2} U^{11}+\ldots+2 h k a^{*} b^{*} U^{12}\right]$

\begin{tabular}{|c|c|c|c|c|c|c|}
\hline & $\mathrm{U}^{11}$ & $\mathrm{U}^{22}$ & $\mathrm{U}^{33}$ & $\mathrm{U}^{23}$ & $\mathrm{U}^{13}$ & $\mathrm{U}^{12}$ \\
\hline $\mathrm{Ru}(1)$ & $16(1)$ & $10(1)$ & $12(1)$ & $4(1)$ & $3(1)$ & 1(1) \\
\hline $\mathrm{Ru}(2)$ & $21(1)$ & $10(1)$ & $12(1)$ & $5(1)$ & $-4(1)$ & $-1(1)$ \\
\hline $\mathrm{Cl}(1)$ & $28(1)$ & $12(1)$ & $13(1)$ & $4(1)$ & $5(1)$ & 2(1) \\
\hline $\mathrm{Cl}(2)$ & $23(1)$ & $15(1)$ & $16(1)$ & $7(1)$ & $3(1)$ & $-2(1)$ \\
\hline $\mathrm{Cl}(3)$ & $24(1)$ & $15(1)$ & $17(1)$ & $8(1)$ & $-3(1)$ & 2(1) \\
\hline $\mathrm{P}(1)$ & $16(1)$ & $12(1)$ & $16(1)$ & $4(1)$ & $4(1)$ & 1(1) \\
\hline $\mathrm{P}(4)$ & $18(1)$ & $12(1)$ & $14(1)$ & $5(1)$ & $-2(1)$ & $-1(1)$ \\
\hline $\mathrm{P}(3)$ & $19(1)$ & $12(1)$ & $17(1)$ & $7(1)$ & $-4(1)$ & $0(1)$ \\
\hline $\mathrm{Cl}(4)$ & $39(1)$ & 11(1) & $17(1)$ & $3(1)$ & $-9(1)$ & $-1(1)$ \\
\hline $\mathrm{Cl}(9)$ & $47(1)$ & $27(1)$ & $34(1)$ & $7(1)$ & $-2(1)$ & $-11(1)$ \\
\hline $\mathrm{Cl}(10)$ & $34(1)$ & $78(2)$ & $48(1)$ & $32(1)$ & $-3(1)$ & $-5(1)$ \\
\hline $\mathrm{Cl}(5)$ & $48(1)$ & $25(1)$ & $35(1)$ & $5(1)$ & $3(1)$ & 11(1) \\
\hline $\mathrm{P}(2)$ & $14(1)$ & $13(1)$ & $14(1)$ & $5(1)$ & $1(1)$ & 1(1) \\
\hline $\mathrm{Cl}(6)$ & $41(1)$ & $75(2)$ & $54(2)$ & $38(1)$ & $3(1)$ & $13(1)$ \\
\hline $\mathrm{Cl}(8)$ & $106(2)$ & $22(1)$ & $51(2)$ & $-3(1)$ & $-12(2)$ & $13(1)$ \\
\hline $\mathrm{Cl}(7)$ & $87(2)$ & $24(1)$ & $74(2)$ & $-2(1)$ & $0(2)$ & $-12(1)$ \\
\hline$C(57)$ & 21(4) & 17(4) & $14(4)$ & $4(3)$ & $0(3)$ & $-4(3)$ \\
\hline $\mathrm{N}(2)$ & $20(3)$ & $13(3)$ & $13(3)$ & $7(3)$ & $0(3)$ & $0(2)$ \\
\hline$C(20)$ & $25(4)$ & $28(5)$ & $25(4)$ & $5(4)$ & $7(4)$ & $-2(3)$ \\
\hline$C(13)$ & $17(4)$ & $14(4)$ & $16(4)$ & $5(3)$ & $-1(3)$ & 1(3) \\
\hline$C(56)$ & $15(4)$ & $13(4)$ & $18(4)$ & $7(3)$ & $-1(3)$ & $-2(3)$ \\
\hline$C(55)$ & $24(4)$ & $15(4)$ & $16(4)$ & $4(3)$ & $4(3)$ & $2(3)$ \\
\hline$C(23)$ & $34(5)$ & $18(4)$ & $18(4)$ & $3(3)$ & $5(4)$ & $-4(3)$ \\
\hline$C(66)$ & $31(5)$ & $27(4)$ & $24(4)$ & $3(4)$ & $-7(4)$ & $7(3)$ \\
\hline$C(79)$ & $17(4)$ & $16(4)$ & $17(4)$ & $7(3)$ & $-2(3)$ & $-6(3)$ \\
\hline$C(63)$ & $20(4)$ & 21(4) & $22(4)$ & $1(4)$ & $0(3)$ & $4(3)$ \\
\hline$C(18)$ & $20(4)$ & $35(5)$ & $22(4)$ & $-1(4)$ & $6(3)$ & $-1(3)$ \\
\hline$C(78)$ & $27(5)$ & 19(4) & $27(4)$ & $4(4)$ & $-2(4)$ & $-6(3)$ \\
\hline$C(19)$ & $17(4)$ & $35(5)$ & $36(5)$ & $12(4)$ & $5(4)$ & $4(3)$ \\
\hline$C(28)$ & $35(5)$ & $23(5)$ & $24(5)$ & $6(4)$ & $2(4)$ & $-15(3)$ \\
\hline $\mathrm{C}(21)$ & $18(4)$ & 16(4) & $11(4)$ & $3(3)$ & $1(3)$ & 1(3) \\
\hline
\end{tabular}




\begin{tabular}{|c|c|c|c|c|c|c|}
\hline $\mathrm{C}(71)$ & $25(4)$ & $25(4)$ & 19(4) & 6(4) & $-4(3)$ & 2(3) \\
\hline$C(52)$ & $27(4)$ & $31(4)$ & $8(4)$ & $-4(3)$ & $1(3)$ & $-7(3)$ \\
\hline$C(82)$ & $26(5)$ & $31(5)$ & $14(4)$ & $10(4)$ & $-2(3)$ & $-2(3)$ \\
\hline $\mathrm{C}(64)$ & $24(4)$ & $30(5)$ & $26(5)$ & $-5(4)$ & $-10(4)$ & $-1(3)$ \\
\hline$C(73)$ & $23(4)$ & $12(4)$ & $20(4)$ & $8(3)$ & $-9(3)$ & 2(3) \\
\hline $\mathrm{C}(69)$ & $36(5)$ & 11(4) & $22(4)$ & $5(3)$ & $0(4)$ & $7(3)$ \\
\hline$C(8)$ & 13(4) & $12(4)$ & $16(4)$ & $-1(3)$ & $1(3)$ & 2(3) \\
\hline$C(62)$ & $25(4)$ & 19(4) & 11(4) & $0(3)$ & $2(3)$ & 1(3) \\
\hline$C(72)$ & 21(4) & $16(4)$ & $20(4)$ & $2(3)$ & $-2(3)$ & $3(3)$ \\
\hline $\mathrm{C}(9)$ & $22(4)$ & $15(4)$ & $23(4)$ & $-2(3)$ & $3(3)$ & $3(3)$ \\
\hline$C(70)$ & $29(5)$ & $30(5)$ & 13(4) & $8(4)$ & $-1(3)$ & $15(3)$ \\
\hline$C(14)$ & $15(4)$ & $16(4)$ & $22(4)$ & $7(3)$ & $-2(3)$ & $-5(3)$ \\
\hline C(59) & $14(4)$ & $7(3)$ & $14(4)$ & $4(3)$ & $1(3)$ & 2(3) \\
\hline$C(17)$ & 19(4) & $17(4)$ & $16(4)$ & $5(3)$ & $3(3)$ & $-2(3)$ \\
\hline$C(50)$ & $22(4)$ & $10(4)$ & $12(4)$ & 4(3) & $2(3)$ & $-1(3)$ \\
\hline$C(24)$ & $28(4)$ & 26(4) & $12(4)$ & $2(3)$ & $2(3)$ & 7(3) \\
\hline$C(5)$ & 21(4) & 11(4) & $13(4)$ & $5(3)$ & $1(3)$ & 2(3) \\
\hline$C(49)$ & $22(4)$ & $12(4)$ & $14(4)$ & $8(3)$ & $-1(3)$ & $-2(3)$ \\
\hline $\mathrm{C}(41)$ & 21(4) & 14(4) & $40(5)$ & $-3(4)$ & $10(4)$ & $0(3)$ \\
\hline$C(37)$ & 13(4) & 13(4) & $25(4)$ & $3(3)$ & $5(3)$ & $3(3)$ \\
\hline$C(10)$ & $25(4)$ & 21(4) & 13(4) & $0(3)$ & $1(3)$ & $-1(3)$ \\
\hline$C(58)$ & 21(4) & 11(4) & $14(4)$ & $7(3)$ & $1(3)$ & $3(3)$ \\
\hline$C(76)$ & $34(5)$ & $10(4)$ & $53(6)$ & $7(4)$ & $-6(4)$ & $-4(3)$ \\
\hline$C(65)$ & $22(5)$ & $32(5)$ & $38(5)$ & $17(4)$ & $0(4)$ & $-2(3)$ \\
\hline$C(12)$ & 21(4) & 13(4) & $12(4)$ & $2(3)$ & $-2(3)$ & $4(3)$ \\
\hline$C(86)$ & $39(5)$ & $25(4)$ & $25(4)$ & 11(4) & $19(4)$ & $8(3)$ \\
\hline$C(74)$ & $25(4)$ & $16(4)$ & 27(4) & $7(4)$ & $-7(4)$ & $5(3)$ \\
\hline C(84) & $20(4)$ & $16(4)$ & $28(4)$ & $13(4)$ & $-2(3)$ & $-2(3)$ \\
\hline$C(80)$ & $14(4)$ & 17(4) & $23(4)$ & $10(3)$ & $-4(3)$ & $-1(3)$ \\
\hline$C(53)$ & $49(6)$ & 19(4) & $22(4)$ & $0(4)$ & $-4(4)$ & $7(4)$ \\
\hline$C(35)$ & $20(4)$ & 19(4) & $22(4)$ & $1(4)$ & $0(3)$ & $-5(3)$ \\
\hline $\mathrm{N}(1)$ & $17(3)$ & 13(3) & $17(3)$ & $3(3)$ & $0(3)$ & $-1(2)$ \\
\hline$C(42)$ & $17(4)$ & 20(4) & $28(4)$ & $5(4)$ & $2(3)$ & $5(3)$ \\
\hline$C(32)$ & 21(4) & $17(4)$ & 21(4) & $5(3)$ & $1(3)$ & $3(3)$ \\
\hline$C(81)$ & $22(4)$ & 20(4) & $22(4)$ & $4(4)$ & $5(3)$ & $-2(3)$ \\
\hline$C(77)$ & $37(5)$ & $22(5)$ & $48(6)$ & $8(4)$ & $5(4)$ & $-7(4)$ \\
\hline
\end{tabular}




\begin{tabular}{|c|c|c|c|c|c|c|}
\hline$C(30)$ & $27(4)$ & 13(4) & $9(4)$ & $-6(3)$ & $3(3)$ & $-3(3)$ \\
\hline$C(31)$ & $13(4)$ & $17(4)$ & $18(4)$ & 10(3) & $4(3)$ & 4(3) \\
\hline$C(83)$ & $30(5)$ & $27(4)$ & $14(4)$ & $15(4)$ & $1(3)$ & 2(3) \\
\hline $\mathrm{C}(27)$ & 19(4) & $37(5)$ & $18(4)$ & $1(4)$ & $8(3)$ & $-10(3)$ \\
\hline$C(26)$ & $22(4)$ & 20(4) & $17(4)$ & $-1(3)$ & $7(3)$ & $-2(3)$ \\
\hline $\mathrm{C}(43)$ & $30(4)$ & 11(4) & $11(4)$ & $1(3)$ & $-4(3)$ & $-3(3)$ \\
\hline$C(2)$ & $40(5)$ & 13(4) & $18(4)$ & $6(3)$ & $13(4)$ & $-5(3)$ \\
\hline$C(15)$ & 21(4) & $9(4)$ & $12(4)$ & $9(3)$ & $0(3)$ & $1(3)$ \\
\hline$C(16)$ & 11(4) & $9(3)$ & $15(4)$ & $5(3)$ & $0(3)$ & $1(3)$ \\
\hline $\mathrm{C}(38)$ & $34(5)$ & $25(5)$ & $44(5)$ & $-3(4)$ & $-6(4)$ & $17(4)$ \\
\hline$C(25)$ & $18(4)$ & 17(4) & $7(4)$ & $1(3)$ & $-3(3)$ & $-3(3)$ \\
\hline $\mathrm{C}(68)$ & $27(4)$ & 19(4) & $12(4)$ & $2(3)$ & $-3(3)$ & $2(3)$ \\
\hline$C(75)$ & 21(4) & $16(4)$ & $35(5)$ & $-2(4)$ & $-15(4)$ & $4(3)$ \\
\hline $\mathrm{C}(11)$ & $18(4)$ & $9(4)$ & $12(4)$ & $6(3)$ & $1(3)$ & $3(3)$ \\
\hline C(29) & $32(5)$ & 14(4) & $24(4)$ & $3(3)$ & $0(4)$ & $-9(3)$ \\
\hline $\mathrm{C}(1)$ & $25(4)$ & 14(4) & $17(4)$ & $-2(3)$ & $6(3)$ & $-2(3)$ \\
\hline$C(85)$ & $35(5)$ & 23(4) & $30(5)$ & $9(4)$ & $-6(4)$ & $-2(3)$ \\
\hline$C(7)$ & $12(4)$ & $12(4)$ & $15(4)$ & $2(3)$ & $-1(3)$ & $0(3)$ \\
\hline$C(4)$ & $27(4)$ & $12(4)$ & $20(4)$ & $9(3)$ & $5(3)$ & $2(3)$ \\
\hline$C(51)$ & $25(4)$ & 13(4) & $14(4)$ & $3(3)$ & $5(3)$ & $2(3)$ \\
\hline$C(34)$ & $24(4)$ & $35(5)$ & $13(4)$ & $5(4)$ & $2(3)$ & $-3(3)$ \\
\hline$C(54)$ & $38(5)$ & $35(5)$ & $12(4)$ & $3(4)$ & $4(4)$ & $-8(4)$ \\
\hline C(39) & $42(6)$ & $48(6)$ & $49(6)$ & $3(5)$ & $-9(5)$ & $28(4)$ \\
\hline $\mathrm{C}(40)$ & $35(5)$ & 13(4) & $54(6)$ & $0(4)$ & $7(5)$ & $10(3)$ \\
\hline $\mathrm{C}(47)$ & 21(4) & $8(4)$ & $17(4)$ & $4(3)$ & $-1(3)$ & 1(3) \\
\hline$C(6)$ & $25(4)$ & $10(4)$ & $22(4)$ & $8(3)$ & $6(3)$ & $7(3)$ \\
\hline$C(45)$ & $45(5)$ & 19(4) & $14(4)$ & $8(3)$ & $-9(4)$ & $3(3)$ \\
\hline$C(36)$ & $14(4)$ & 20(4) & $20(4)$ & $10(3)$ & $6(3)$ & $0(3)$ \\
\hline $\mathrm{C}(60)$ & 21(4) & $12(4)$ & $16(4)$ & $4(3)$ & $-1(3)$ & 2(3) \\
\hline$C(3)$ & $50(5)$ & $15(4)$ & 21(4) & $13(4)$ & $4(4)$ & $-1(4)$ \\
\hline$C(67)$ & $28(4)$ & 12(4) & $13(4)$ & $2(3)$ & $2(3)$ & 2(3) \\
\hline $\mathrm{C}(61)$ & $26(4)$ & $18(4)$ & $21(4)$ & $-1(3)$ & $0(3)$ & $0(3)$ \\
\hline$C(22)$ & $29(5)$ & $24(4)$ & $12(4)$ & $-2(3)$ & $-3(3)$ & $-2(3)$ \\
\hline $\mathrm{C}(48)$ & $30(4)$ & $15(4)$ & $18(4)$ & $8(3)$ & $-6(3)$ & $-3(3)$ \\
\hline$C(46)$ & $37(5)$ & 13(4) & $15(4)$ & $4(3)$ & $-1(4)$ & $-3(3)$ \\
\hline$C(33)$ & $29(5)$ & $30(5)$ & $28(5)$ & $15(4)$ & $4(4)$ & $-1(3)$ \\
\hline
\end{tabular}


Table S35. Hydrogen coordinates ( $\left.\times 10^{4}\right)$ and isotropic displacement parameters $\left(\AA^{2} \times 10^{3}\right)$ for $5 \mathbf{a}$.

\begin{tabular}{|c|c|c|c|c|}
\hline & $\mathrm{x}$ & $\mathrm{y}$ & $\mathrm{z}$ & $\mathrm{U}(\mathrm{eq})$ \\
\hline $\mathrm{H}(57)$ & 10571 & 1700 & 9167 & 21 \\
\hline $\mathrm{H}(20 \mathrm{~A})$ & 2071 & 4931 & 8196 & 40 \\
\hline $\mathrm{H}(20 \mathrm{~B})$ & 1446 & 5358 & 8864 & 40 \\
\hline $\mathrm{H}(20 \mathrm{C})$ & 2490 & 5038 & 9019 & 40 \\
\hline $\mathrm{H}(55)$ & 11625 & 380 & 7357 & 21 \\
\hline $\mathrm{H}(23 \mathrm{~A})$ & 3474 & 4062 & 6748 & 35 \\
\hline $\mathrm{H}(23 \mathrm{~B})$ & 4560 & 3774 & 6781 & 35 \\
\hline $\mathrm{H}(23 \mathrm{C})$ & 4008 & 3713 & 6006 & 35 \\
\hline $\mathrm{H}(66 \mathrm{~A})$ & 12796 & 80 & 8119 & 41 \\
\hline $\mathrm{H}(66 \mathrm{~B})$ & 12328 & 46 & 8895 & 41 \\
\hline $\mathrm{H}(66 \mathrm{C})$ & 13385 & 435 & 8861 & 41 \\
\hline $\mathrm{H}(18 \mathrm{~A})$ & 1944 & 6668 & 9556 & 40 \\
\hline $\mathrm{H}(18 \mathrm{~B})$ & 2875 & 7134 & 9332 & 40 \\
\hline $\mathrm{H}(18 \mathrm{C})$ & 3003 & 6371 & 9702 & 40 \\
\hline $\mathrm{H}(78)$ & 4423 & -750 & 8092 & 29 \\
\hline $\mathrm{H}(19 \mathrm{~A})$ & 1856 & 6129 & 7621 & 43 \\
\hline $\mathrm{H}(19 \mathrm{~B})$ & 2274 & 6976 & 8028 & 43 \\
\hline $\mathrm{H}(19 \mathrm{C})$ & 1318 & 6601 & 8317 & 43 \\
\hline $\mathrm{H}(28)$ & 10987 & 7192 & 6090 & 33 \\
\hline $\mathrm{H}(71)$ & 3175 & 665 & 5938 & 27 \\
\hline $\mathrm{H}(52 \mathrm{~A})$ & 8880 & 342 & 6085 & 35 \\
\hline $\mathrm{H}(52 \mathrm{~B})$ & 8739 & -456 & 6405 & 35 \\
\hline $\mathrm{H}(52 \mathrm{C})$ & 9218 & -480 & 5622 & 35 \\
\hline $\mathrm{H}(82)$ & 6081 & 624 & 10325 & 28 \\
\hline $\mathrm{H}(64 \mathrm{~A})$ & 11914 & 1370 & 9726 & 42 \\
\hline $\mathrm{H}(64 \mathrm{~B})$ & 12135 & 2174 & 9431 & 42 \\
\hline $\mathrm{H}(64 \mathrm{C})$ & 13007 & 1645 & 9615 & 42 \\
\hline $\mathrm{H}(69)$ & 5040 & 2433 & 6858 & 27 \\
\hline $\mathrm{H}(62 \mathrm{~A})$ & 9237 & 1895 & 9857 & 28 \\
\hline $\mathrm{H}(62 \mathrm{~B})$ & 8691 & 1049 & 9643 & 28 \\
\hline
\end{tabular}




\begin{tabular}{|c|c|c|c|c|}
\hline $\mathrm{H}(62 \mathrm{C})$ & 8081 & 1836 & 9812 & 28 \\
\hline $\mathrm{H}(72)$ & 4045 & -224 & 6483 & 23 \\
\hline $\mathrm{H}(9 \mathrm{~A})$ & 5620 & 7756 & 8874 & 31 \\
\hline $\mathrm{H}(9 \mathrm{~B})$ & 6117 & 7542 & 8093 & 31 \\
\hline $\mathrm{H}(9 \mathrm{C})$ & 6773 & 7782 & 8823 & 31 \\
\hline $\mathrm{H}(70)$ & 3703 & 1989 & 6097 & 28 \\
\hline $\mathrm{H}(14)$ & 3109 & 5266 & 7336 & 21 \\
\hline $\mathrm{H}(24 \mathrm{~A})$ & 3739 & 4944 & 5484 & 33 \\
\hline $\mathrm{H}(24 \mathrm{~B})$ & 4017 & 5793 & 5966 & 33 \\
\hline $\mathrm{H}(24 \mathrm{C})$ & 3138 & 5275 & 6198 & 33 \\
\hline $\mathrm{H}(41)$ & 10004 & 2443 & 5763 & 31 \\
\hline $\mathrm{H}(10 \mathrm{~A})$ & 6905 & 6849 & 9708 & 30 \\
\hline $\mathrm{H}(10 \mathrm{~B})$ & 6286 & 6033 & 9565 & 30 \\
\hline $\mathrm{H}(10 \mathrm{C})$ & 5754 & 6855 & 9772 & 30 \\
\hline $\mathrm{H}(76)$ & 3477 & -2840 & 6878 & 39 \\
\hline $\mathrm{H}(65 \mathrm{~A})$ & 12595 & 2134 & 8125 & 45 \\
\hline $\mathrm{H}(65 \mathrm{~B})$ & 12936 & 1317 & 7656 & 45 \\
\hline $\mathrm{H}(65 \mathrm{C})$ & 13552 & 1738 & 8366 & 45 \\
\hline $\mathrm{H}(12)$ & 4358 & 6632 & 9111 & 19 \\
\hline H(74) & 5696 & -1603 & 6179 & 27 \\
\hline $\mathrm{H}(84)$ & 6778 & -788 & 8431 & 24 \\
\hline $\mathrm{H}(80)$ & 5085 & 1079 & 8387 & 21 \\
\hline $\mathrm{H}(53 \mathrm{~A})$ & 10564 & -1155 & 6005 & 46 \\
\hline $\mathrm{H}(53 \mathrm{~B})$ & 10205 & -1114 & 6827 & 46 \\
\hline $\mathrm{H}(53 \mathrm{C})$ & 11258 & -773 & 6685 & 46 \\
\hline $\mathrm{H}(35)$ & 9804 & 6415 & 9533 & 25 \\
\hline $\mathrm{H}(42)$ & 8989 & 3512 & 5980 & 25 \\
\hline $\mathrm{H}(32)$ & 8072 & 4184 & 8314 & 23 \\
\hline $\mathrm{H}(81)$ & 5178 & 1399 & 9667 & 26 \\
\hline $\mathrm{H}(77)$ & 3383 & -1868 & 7931 & 42 \\
\hline $\mathrm{H}(30)$ & 8755 & 6576 & 7254 & 21 \\
\hline $\mathrm{H}(83)$ & 6857 & -472 & 9708 & 27 \\
\hline $\mathrm{H}(27)$ & 11511 & 5886 & 5903 & 30 \\
\hline $\mathrm{H}(26)$ & 10617 & 4920 & 6358 & 24 \\
\hline $\mathrm{H}(43)$ & 6003 & 23 & 5408 & 21 \\
\hline $\mathrm{H}(2)$ & 8917 & 6000 & 4616 & 28 \\
\hline
\end{tabular}




\begin{tabular}{|c|c|c|c|c|}
\hline $\mathrm{H}(38)$ & 10308 & 4273 & 7948 & 42 \\
\hline $\mathrm{H}(68)$ & 5947 & 1536 & 7363 & 23 \\
\hline $\mathrm{H}(75)$ & 4647 & -2721 & 6025 & 30 \\
\hline $\mathrm{H}(29)$ & 9630 & 7533 & 6786 & 28 \\
\hline $\mathrm{H}(1)$ & 8535 & 5040 & 5284 & 23 \\
\hline $\mathrm{H}(7 \mathrm{~A})$ & 7275 & 5797 & 8559 & 16 \\
\hline $\mathrm{H}(7 \mathrm{~B})$ & 7590 & 6595 & 8281 & 16 \\
\hline $\mathrm{H}(4)$ & 7170 & 7479 & 5881 & 23 \\
\hline $\mathrm{H}(34)$ & 9023 & 5580 & 10201 & 29 \\
\hline $\mathrm{H}(54 \mathrm{~A})$ & 10496 & 920 & 5973 & 43 \\
\hline $\mathrm{H}(54 \mathrm{~B})$ & 10801 & 89 & 5506 & 43 \\
\hline $\mathrm{H}(54 \mathrm{C})$ & 11441 & 502 & 6206 & 43 \\
\hline $\mathrm{H}(39)$ & 11309 & 3183 & 7745 & 56 \\
\hline $\mathrm{H}(40)$ & 11159 & 2272 & 6652 & 41 \\
\hline $\mathrm{H}(6 \mathrm{~A})$ & 6012 & 6672 & 6684 & 22 \\
\hline $\mathrm{H}(6 \mathrm{~B})$ & 6947 & 7061 & 7145 & 22 \\
\hline $\mathrm{H}(45)$ & 6374 & 2226 & 4989 & 31 \\
\hline $\mathrm{H}(36)$ & 9753 & 6123 & 8254 & 20 \\
\hline $\mathrm{H}(60 \mathrm{~A})$ & 7279 & 1628 & 8377 & 20 \\
\hline $\mathrm{H}(60 \mathrm{~B})$ & 7569 & 831 & 8649 & 20 \\
\hline $\mathrm{H}(3)$ & 8169 & 7231 & 4879 & 33 \\
\hline $\mathrm{H}(61 \mathrm{~A})$ & 9318 & 2815 & 8994 & 34 \\
\hline $\mathrm{H}(61 \mathrm{~B})$ & 8165 & 2814 & 8922 & 34 \\
\hline $\mathrm{H}(61 \mathrm{C})$ & 8797 & 2608 & 8202 & 34 \\
\hline $\mathrm{H}(22 \mathrm{~A})$ & 5884 & 4499 & 6322 & 34 \\
\hline $\mathrm{H}(22 \mathrm{~B})$ & 5714 & 5310 & 6039 & 34 \\
\hline $\mathrm{H}(22 \mathrm{C})$ & 5328 & 4488 & 5557 & 34 \\
\hline $\mathrm{H}(48 \mathrm{~A})$ & 7819 & 2095 & 7236 & 25 \\
\hline $\mathrm{H}(48 \mathrm{~B})$ & 8704 & 1727 & 6759 & 25 \\
\hline $\mathrm{H}(46)$ & 7504 & 2489 & 5963 & 26 \\
\hline $\mathrm{H}(33)$ & 8163 & 4450 & 9590 & 33 \\
\hline $\mathrm{H}(44)$ & 5581 & 967 & 4742 & 26 \\
\hline
\end{tabular}

